\title{
On Special Optical Modes and Thermal Issues in Advanced Gravitational Wave Interferometric Detectors
}

\author{
Jean-Yves Vinet \\ Observatoire de la Côte d'Azur (ARTEMIS) \\ Université de Nice-Sophia Antipolis \\ 06304 Nice, France \\ email: Jean-Yves.VINET@obs-nice.fr

\section{Living Reviews in Relativity ISSN 1433-8351}

Accepted on 14 May 2009

Published on 17 July 2009

\begin{abstract}
The sensitivity of present ground-based gravitational wave antennas is too low to detect many events per year. It has, therefore, been planned for years to build advanced detectors allowing actual astrophysical observations and investigations. In such advanced detectors, one major issue is to increase the laser power in order to reduce shot noise. However, this is useless if the thermal noise remains at the current level in the $100 \mathrm{~Hz}$ spectral region, where mirrors are the main contributors. Moreover, increasing the laser power gives rise to various spurious thermal effects in the same mirrors. The main goal of the present study is to discuss these issues versus the transverse structure of the readout beam, in order to allow comparison. A number of theoretical studies and experiments have been carried out, regarding thermal noise and thermal effects. We do not discuss experimental problems, but rather focus on some theoretical results in this context about arbitrary order Laguerre-Gauss beams, and other "exotic" beams.
\end{abstract}

This review is licensed under a Creative Commons Attribution-Non-Commercial-NoDerivs 3.0 Germany License. http://creativecommons.org/licenses/by-nc-nd/3.0/de/ 


\section{Imprint / Terms of Use}

Living Reviews in Relativity is a peer reviewed open access journal published by the Max Planck Institute for Gravitational Physics, Am Mühlenberg 1, 14476 Potsdam, Germany. ISSN 1433-8351.

This review is licensed under a Creative Commons Attribution-Non-Commercial-NoDerivs 3.0 Germany License: http://creativecommons.org/licenses/by-nc-nd/3.0/de/

Because a Living Reviews article can evolve over time, we recommend to cite the article as follows:

Jean-Yves Vinet,

"On Special Optical Modes and Thermal Issues in Advanced Gravitational Wave Interferometric Detectors",

Living Rev. Relativity, 12, (2009), 5. [Online Article]: cited [<date $>$ ], http://www.livingreviews.org/lrr-2009-5

The date given as $<$ date $>$ then uniquely identifies the version of the article you are referring to.

\section{Article Revisions}

Living Reviews supports two different ways to keep its articles up-to-date:

Fast-track revision A fast-track revision provides the author with the opportunity to add short notices of current research results, trends and developments, or important publications to the article. A fast-track revision is refereed by the responsible subject editor. If an article has undergone a fast-track revision, a summary of changes will be listed here.

Major update A major update will include substantial changes and additions and is subject to full external refereeing. It is published with a new publication number.

For detailed documentation of an article's evolution, please refer always to the history document of the article's online version at http: //www.livingreviews.org/lrr-2009-5. 


\section{Contents}

1 Introduction $r$

2 Modes of Fabry-Pérot Cavities and Readout Beams 9

2.1 Laguerre-Gauss beams . . . . . . . . . . . . . . . . . . . . . . . . . . . . . . . . . . . . . . . . . . .

2.2 Mesa and flat beams . . . . . . . . . . . . . . . . . . . . . . . . . . . . . . . . . . . . . . . . . . . . . .

2.3 Other exotic modes . . . . . . . . . . . . . . . . . . . . . 14

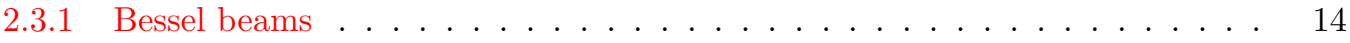

2.3.2 Conical-mirror or Gauss-Bessel beams . . . . . . . . . . . . . . 15

3 Heating and Thermal Effects in the Steady State $\quad 17$

3.1 Steady temperature field . . . . . . . . . . . . . . . . . . . 17

3.1 Coating absorption . . . . . . . . . . . . . . . 17

3.1 .2 Bulk absorption . . . . . . . . . . . . . . . . . 19

3.1.3 Fourier-Bessel expansion of the readout beam intensity . . . . . . . . . 20

3.1 .4 Numerical results on temperature fields . . . . . . . . . . . . . . . 21

3.2 Steady thermal lensing . . . . . . . . . . . . . . . . . . . . . . 30

3.2.1 Thermal lensing from coating absorption . . . . . . . . . . . . . . 32

3.2.2 Thermal lens from bulk absorption . . . . . . . . . . . . . . . 32

3.2.3 Equivalent paraboloid . . . . . . . . . . . . . . . . . 32

3.2 .4 Coupling losses . . . . . . . . . . . . . . . . . . . 35

3.2 .5 Numerical results . . . . . . . . . . . . . . . . . . . . . . . 37

3.3 Thermal distortions in the steady state . . . . . . . . . . . . . . . . . 39

3.3.1 Thermal expansion from thermalization on the coating . . . . . . . . . 39

3.3.2 Thermal expansion from internal absorption . . . . . . . . . . . . . 45

3.4 Expansion on Zernike polynomials . . . . . . . . . . . . . . . . . . . 51

4 On Thermal Compensation Systems $\quad \mathbf{5 3}$

4.1 Heating the rear face of a mirror . . . . . . . . . . . . . . . . . . 53

4.2 Simple model of a radiator . . . . . . . . . . . . . . . . . . . . . . 54

4.3 Axicon systems . . . . . . . . . . . . . . . . . . . . . . . . . 57

$4.4 \mathrm{CO}_{2}$ laser compensation by scanning . . . . . . . . . . . . . . . . 59

5 Heating in the Quasistatic Regime: Heating from Cold $\quad 61$

5.1 Transient temperature field . . . . . . . . . . . . . . . . 6 61

5.1 Transient temperature from coating absorption . . . . . . . . . . . 64

5.1 .2 Transient temperature from bulk absorption . . . . . . . . . . . . 64

5.2 Transient thermal distortions . . . . . . . . . . . . . . . . . 68

5.2 .1 Case of coating absorption . . . . . . . . . . . . . . . . . . . 69

5.2 .2 Case of bulk absorption ....................... 73

6 Heating and Thermal Effects in the Dynamic Regime: Transfer Functions 77

6.1 Temperature fields and thermal lensing . . . . . . . . . . . . . . . . . 77

6.1.1 Coating absorption ..................... 77

6.1 .2 Bulk absorption ....................... . . 78

6.2 Equivalent displacement noise . . . . . . . . . . . . . . . . . . . . 78 
7 Dynamic Surface Distortion $\quad \mathbf{8 0}$

7.1 Dynamic surface distortion caused by coating absorption . . . . . . . . . . . . 82

7.1 .1 Mean displacement . . . . . . . . . . . . . . . . . . . . 83

7.1 .2 Under cutoff regime . . . . . . . . . . . . . . . . . . . 83

8 Brownian Thermal Noise $\quad \mathbf{8 4}$

8.1 The Fluctuation-Dissipation theorem and Levin's generalized coordinate method . 84

8.2 Infinite mirrors noise in the substrate . . . . . . . . . . . . . . . 85

8.3 Infinite mirrors, noise in coating . . . . . . . . . . . . . 88

8.3.1 Coating Brownian thermal noise: LG modes . . . . . . . . . . . . . . 90

8.3.2 Coating Brownian thermal noise: Flat modes . . . . . . . . . . . . . . 91

8.4 Finite mirrors . . . . . . . . . . . . . . . . . . . . . . . . . . . . 91

8.4.1 Equilibrium equations . . . . . . . . . . . . . . . . . . . . . . 92

8.4 .2 Boundary conditions . . . . . . . . . . . . . . . . . 92

8.4 .3 Strain energy . . . . . . . . . . . . . . . . . . . . . 95

8.4.4 Explicit displacement and strain tensor _ . . . . . . . . . . . . . 97

8.5 Coating Brownian thermal noise: finite mirrors . . . . . . . . . . . . . . 103

9 Thermoelastic Noise 105

9.1 Introduction . . . . . . . . . . . . . . . . . . . . . . . . 105

9.2 Case of infinite mirrors . . . . . . . . . . . . . . . . . . . . . . 106

9.2 .1 Gaussian beams . . . . . . . . . . . . . . . . . . . . . . 108

9.2 .2 Flat beams . . . . . . . . . . . . . . . . . . . . . . . . . . 109

9.2 .3 Thermoelastic noise in the coating . . . . . . . . . . . . . . . . . 109

9.2 .4 Scaling laws . . . . . . . . . . . . . . . . . . . . . . 110

9.2 .5 Numerical results . . . . . . . . . . . . . . . . . . . . . . . . . . 110

9.3 Case of finite mirrors . . . . . . . . . . . . . . . . . . . . . . . . . 111

9.3 .1 Case of the bulk material . . . . . . . . . . . . . . . . . 112

9.3 .2 Case of the coatings . . . . . . . . . . . . . . . . . 117

9.3 .3 Numerical results . . . . . . . . . . . . . . . . . . . . . . . . . . 117

10 Generation of High Order Modes $\quad 118$

$\begin{array}{ll}11 \text { Conclusion } & 120\end{array}$

$\begin{array}{ll}\text { References } & 121\end{array}$

\section{List of Tables}

1 Physical constants used in this paper . . . . . . . . . . . . . . . . . 23

2 Thermal lensing from coating and bulk absorption (abs.) . . . . . . . . . . . 37

3 Thermal lensing curvature radii $\left(R_{c}\right)$ for LG modes having $1 \mathrm{ppm}$ clipping losses, and associated coupling losses (L) in the weak power approximation [Equation (3.83)] (mirror diameter: $35 \mathrm{~cm}) \ldots \ldots \ldots \ldots \ldots$

4 Curvature radii from thermal expansion due to coating absorption for modes having $1 \mathrm{ppm}$ clipping losses . . . . . . . . . . . . . . . . . . . . . 44

5 Curvature radii from thermal expansion due to bulk absorption for modes having $1 \mathrm{ppm}$ clipping losses . . . . . . . . . . . . . . . . . . . . . . . 49

6 Thermal aberrations from coating and bulk absorption . . . . . . . . . . 50

$7 \quad$ Zernike coefficients $c_{n}$ for $\mathrm{LG}_{00} w=2 \mathrm{~cm} \ldots \ldots \ldots \ldots 2$ 
$8 \quad$ Zernike coefficients $c_{n}$ for $\mathrm{LG}_{55} w=3.5 \mathrm{~cm} \ldots \ldots \ldots 52$

9 Zernike coefficients $c_{n}$ for the mesa mode . . . . . . . . . . . . . . . 53

10 Thermal compensation with a heating ring: $\mathrm{LG}_{0,0}$ mode of $w=2 \mathrm{~cm}$. . . . . . . 55

11 Thermal compensation with a heating ring: $\mathrm{LG}_{5,5}$ mode of $w=3.5 \mathrm{~cm}$. . . . . . . 57

12 Thermal compensation with an axicon system . . . . . . . . . . . 58

13 Thermal compensation with a scanning $\mathrm{CO}_{2}$ beam for $\mathrm{LG}_{00}$ mode $(w=2 \mathrm{~cm})$. . 60

14 Zernike coefficients for three TCS systems compensating $\mathrm{LG}_{00}$ mode $(w=2 \mathrm{~cm})$. 61

15 Some numerical values of $g_{0, n, m} \ldots \ldots \ldots \ldots$. . . . . . . . . . . . . . . . . . . . . . . . . . . . .

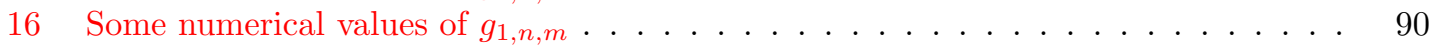

17 Comparison infinite/finite mirror Strain Energy (SE) . . . . . . . . . . . . . . . . 104

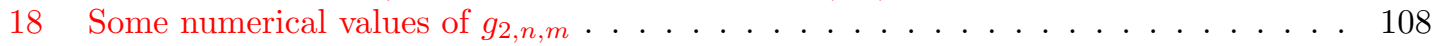

19 Some numerical values of $g_{3, n, m} \ldots \ldots \ldots \ldots \ldots$

20 Some values of $\varpi_{n} \ldots \ldots \ldots \ldots$. . . . . . . . . . . . . . . 110 



\section{Introduction}

Gravitational waves (GWs) are a prediction of Einstein's general theory of relativity, which extends the theory of gravitation by renouncing the instantaneous action at a distance that was shocking to Isaac Newton himself and had already became unacceptable after the special theory of relativity. The gravitational interaction is now carried by a wave messenger at the speed of light, a gravitational wave. However, the efficiency of the conversion of any kind of energy into gravitational radiation is extremely weak, so that emitting and/or detecting such waves has for decades been considered well outside experimental possibilities. The situation changed after the technological expansion in the 1960s. Joseph Weber was the first to propose an experiment aiming to detect GWs of astrophysical origin. However, the initial Weber experiment was still too simple to detect anything of astrophysical interest. This motivated theorists to work out more accurate estimates of the GW signals produced by astrophysical cataclysms such as supernovae, binary coalescences, fast spinning neutron stars etc... Readers interested in this part of the history of the field can consult the review by Thorne [37]. Is was soon noted that optical interferometers of the Michelson type had exactly the right topology with respect to the gravitational wave polarization, had a large potential sensitivity and were able to produce electrical signals analog to the gravitational waveforms, being intrinsically wide-band transducers. After several prototypes of various sizes were built (U.S.A., U.K., Germany), and following the pioneering work of Ronald Drever of Caltech, Rainer Weiss of MIT was the first to study the technological issues specific to large size interferometric antennas and to determine the general principles of ground based antennas. This was the seed of the LIGO project in the U.S.A. [27], of the British-German GEO, unfortunately aborted, and of the French-Italian Virgo [41]. Despite these efforts and after construction of kilometer size antennas, GWs have yet to be detected because of the still too low sensitivity of present antennas (LIGO, Virgo). It was foreseen from the beginning that technological breakthroughs would allow the sensitivity to be enhanced in the near future. This is the present situation, and the R\&D of "advanced detectors" has already begun. One aspect of these advanced detectors is an improved use of light for reading the tiny apparent variations of distances between test masses.

Ground-based interferometers for GW detection are made of silica pieces (the substrates of the mirrors) hanging in a vacuum. Detection of GWs requires the continuous measurement of the flight time of photons between two mirrors facing each other, or, in other words, the reflected phase off a Fabry-Pérot cavity. A passing GW is expected to have a differential effect on the phases of two orthogonal cavities. This is why the Michelson configuration is well adapted to GW antennas. It is classically shown that the sensitivity of a Michelson interferometer ultimately depends on the square root of the light source's power. This is a strong reason to increase the input laser power. However, there are at least four issues to solve before such an improvement can be made. The first is that, even with high quality materials, a fraction of the power is absorbed by the material (either in the bulk or on the coating); this gives rise to a source of heat at the surface or in the bulk, and there is consequently a temperature field in the material, which results in turn in a refractive index field and a thermal distortion of the substrate. These defects cause mismatching of the interferometer, and therefore, already in the present status of LIGO-Virgo, require complex thermal compensation systems. Before increasing the incident power, some new ideas would be welcome. The second issue comes from the fact that in the region of $100 \mathrm{~Hz}$, the sensitivity is not limited by shot noise, but rather by the thermal noise of mirrors. Mirror substrates may be viewed as elastodynamical oscillators, whose modes are excited at room temperature resulting in a fluctuating reflecting face. Increasing the laser power will be of no use in this strategic spectral region unless a means of reducing the effect of thermal noise is found. There is still another source of noise, called thermoelastic, due to temperature fluctuations in the material. The fourth issue is the effect of radiation pressure on the suspended mirrors. Increasing the laser power will cause increasing fluctuations in the radiation pressure, so that there is an optimum in the laser 
power, dependant on the cavity parameters, giving the standard quantum limit. In the context of the R\&D of advanced detectors, several ways of reducing the thermal noise have been proposed: using new high-Q materials [34], cooling to cryogenic temperatures [39] or active correction [10]. Changing the geometry of the readout beam, in order to reduce the optical coupling with surface fluctuations, has also been proposed. Regarding this track, there was a proposal $[11,40]$ to go towards nonspherical mirrors generating a more-or-less homogeneous light-intensity profile. There was another proposal [31] in the same spirit but keeping spherical mirrors and using high-order Gaussian modes. Some other proposals are also considered.

Thermal effects, the various thermal noises (Brownian, thermoelastic, thermorefractive) have been extensively studied and reported in the literature. We focus here on their dependence to the transverse structure of the optical readout beam and try particularly to give general formulas for arbitrary order Laguerre-Gauss modes.

Further material will be added to the present review with coming developments, especially regarding experimental results. But we think it is useful to present already available results during the present R\&D phase. 


\section{Modes of Fabry-Pérot Cavities and Readout Beams}

It is well known that a Fabry-Pérot cavity has eigenmodes corresponding to eigenfrequencies determined by its length and geometries defined by the shape of the mirrors. It is also well known that cavities with spherical mirrors are resonant for Hermite-Gauss or Laguerre-Gauss modes. Recently more exotic shapes have been proposed in order to reduce the thermal noise.

\subsection{Laguerre-Gauss beams}

Because we are developing models based on axisymmetry, we pay special attention to cylindrical coordinates, and consequently to the Laguerre-Gauss family of modes. If $z$ is the coordinate along the optical axis, $r$ the radial coordinate and $\varphi$ the azimuthal, a Laguerre-Gauss mode $\left(\mathrm{LG}_{n, m}\right)$ of parameter $w$ has the following complex amplitude at $z$ (the wavelength is $\lambda$ and $k \equiv 2 \pi / \lambda$ ):

$$
\Psi_{m}^{(n)}(r, \varphi, z)=R_{m}^{(n)}(r)^{1 / 2}\left\{\begin{array}{c}
\cos (n \varphi) \\
\sin (n \varphi)
\end{array}\right\} \mathrm{e}^{i k z} \mathrm{e}^{i(2 m+n+1) G(z)}
$$

where $G(z)$ is the Gouy phase,

$$
G(z)=\tan ^{-1}\left(z / z_{R}\right)
$$

$z_{R} \equiv \pi w_{0}^{2} / \lambda$ is the Rayleigh parameter, and $w=w_{0} \sqrt{1+\left(z / z_{R}\right)^{2}}$ is the beam width at $z$. Parameter $w_{0}$ represents the minimum width (waist) of the beam, localized at abscissa $z=0$. The normalized radial function $R_{m}^{(n)}(r)$ is given by

$$
R_{m}^{(n)}(r, \varphi, z)=\frac{4}{\left(1+\delta_{n, 0}\right) \pi w^{2}} \frac{m !}{(n+m) !}\left(\frac{2 r^{2}}{w^{2}}\right)^{n} L_{m}^{(n)}\left(\frac{2 r^{2}}{w^{2}}\right)^{2} \exp \left(-\frac{2 r^{2}}{w^{2}}\right) .
$$

$L_{m}^{(n)}(X)$ are the generalized Laguerre polynomials. Figure 1 shows the intensity pattern of a nonaxisymmetric Laguerre-Gauss mode of cosine angular parity. Obviously the origin of angles is arbitrary, so that we can replace the cosine by a sine, and even combine a cosine mode with a sine mode to obtain a mode having an axisymmetric intensity. For such an axisymmetric (in intensity) mode, the normalization is slightly different, so that

$$
R_{m}^{(n)}(r, \varphi, z)=\frac{2}{\pi w^{2}} \frac{m !}{(n+m) !}\left(\frac{2 r^{2}}{w^{2}}\right)^{n} L_{m}^{(n)}\left(\frac{2 r^{2}}{w^{2}}\right)^{2} \exp \left(-\frac{2 r^{2}}{w^{2}}\right) .
$$

See Figure 2 for the intensity pattern of such a readout beam.

\subsection{Mesa and flat beams}

We shall see in a following Section 8 that thermal noise is reduced by widening the beam on a mirror in such a way that the fluctuations of the surface are significantly cancelled by averaging on the readout beam cross section. A way of obtaining almost "flat" beam profiles was proposed by a Caltech team led by Thorne and O'Shaughnessy $[12,33,11]$. To understand the proposal, we start from a fundamental mode $\mathrm{LG}_{0,0}$ at its waist $(z=0)$

$$
\phi(x, y, 0)=\sqrt{\frac{2}{\pi w_{0}^{2}}} \exp \left(-r^{2} / w_{0}^{2}\right) \quad\left(r^{2}=x^{2}+y^{2}\right),
$$

and we take the convolution product with the characteristic function of a centered disk $\Delta$ of radius $b_{f}$

$$
\Psi(x, y, 0)=\frac{\kappa}{\pi b_{f}^{2}} \int_{\Delta} \phi\left(x-x_{0}, y-y_{0}, 0\right) d x_{0} d y_{0},
$$




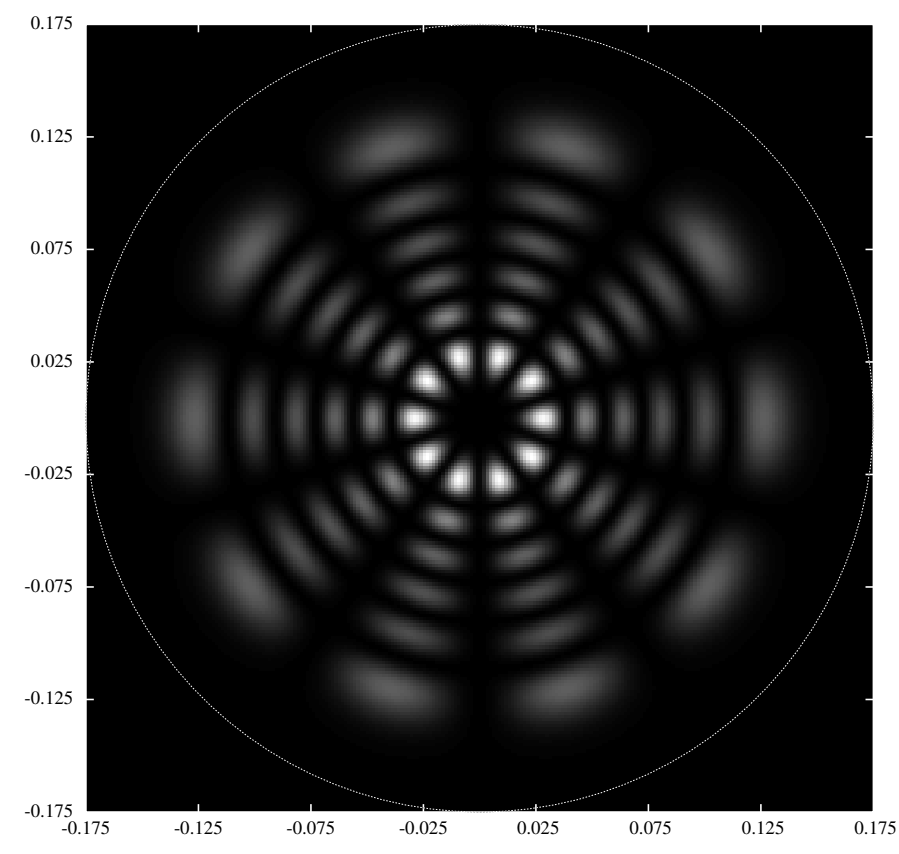

Figure 1: Intensity distribution in an $\mathrm{LG}_{5,5}$ mode of width parameter $w=3.5 \mathrm{~cm}$. Dashed circle: edge of a mirror of radius $17.5 \mathrm{~cm}$. 


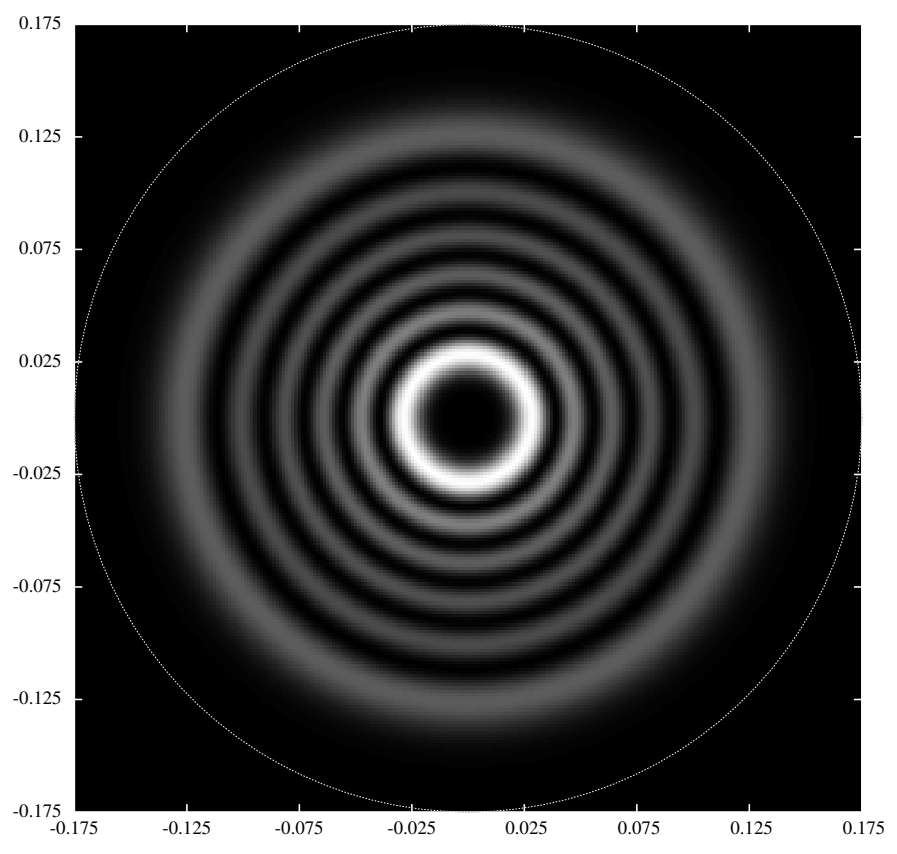

Figure 2: Intensity distribution in an axisymmetric $\mathrm{LG}_{5,5}$ mode of width parameter $w=3.5 \mathrm{~cm}$. Dashed circle: edge of a mirror of radius $17.5 \mathrm{~cm}$. 
where $\kappa$ is a constant to be determined by normalization. This mode of construction allows one to compute the propagated mode. Because $\Psi$ is a linear combination of modes, its propagated value is nothing but the same combination of propagated elementary modes

$$
\Psi(x, y, z)=\frac{\kappa}{\pi b_{f}^{2}} \int_{\Delta} \phi\left(x-x_{0}, y-y_{0}, z\right) d x_{0} d y_{0}
$$

so that the mode is defined at any abscissa $z$ by

$$
\begin{aligned}
\Psi(x, y, z)= & \frac{\kappa}{\pi b_{f}^{2}} \sqrt{\frac{2}{\pi w^{2}}} \exp \left(-i \tan ^{-1}\left(z / z_{R}\right)\right) \\
& \int_{\Delta} \exp \left\{-\frac{Z}{w^{2}}\left[\left(x-x_{0}\right)^{2}+\left(y-y_{0}\right)^{2}\right]\right\} d x_{0} d y_{0}
\end{aligned}
$$

where $z_{R} \equiv \pi w_{0}^{2} / \lambda$ is the Rayleigh parameter, $Z \equiv 1-i z / z_{R}$ and $w \equiv w_{0} \sqrt{Z \bar{Z}}$. After some algebra, the result being axisymmetric, this is equivalent to

$$
\begin{aligned}
\Psi(r, z)= & \frac{2 \kappa}{b_{f}^{2}} \sqrt{\frac{2 w^{2}}{\pi}} \exp \left(-i \tan ^{-1}\left(z / z_{R}\right)\right) \\
& \int_{0}^{b / w} \exp \left[-Z(r / w-x)^{2}\right] \exp (-2 Z r x / w) I_{0}(2 Z r x / w) x d x .
\end{aligned}
$$

Normalization is easier to compute in the Fourier space. We have, after the Plancherel theorem

$$
\|\Psi\|^{2}=2 \pi \int_{0}^{\infty}|\Psi(r, z)|^{2} r d r=\frac{1}{2 \pi} \int_{0}^{\infty}|\tilde{\Psi}(\rho, z)|^{2} \rho d \rho .
$$

Now, the Fourier transform of the mode is nothing but the product of the Fourier transform of the elementary mode by the Fourier transform of the characteristic function of the disk. The Fourier transform of the mode at $z=0$ is

$$
\tilde{\phi}(\rho, 0)=\sqrt{2 \pi w_{0}^{2}} \exp \left[-\frac{w_{0}^{2} \rho^{2}}{4}\right],
$$

whereas the Fourier transform of the disk is

$$
\tilde{\mathcal{F}}_{\Delta}(\rho)=\frac{2 J_{1}\left(b_{f} \rho\right)}{b_{f} \rho}
$$

where $J_{1}(x)$ is a Bessel function. Thus, we have

$$
\|\Psi\|^{2}=\frac{\kappa^{2}}{2 \pi} \int_{0}^{\infty}\left|\tilde{\phi}(\rho, 0) \tilde{\mathcal{F}}_{\Delta}(\rho)\right|^{2} \rho d \rho=\frac{4 w_{0}^{2} \kappa^{2}}{b_{f}^{2}} \int_{0}^{\infty} J_{1}(x)^{2} \exp \left[-\frac{w_{0}^{2} x^{2}}{2 b_{f}^{2}}\right] \frac{d x}{x}=\frac{2 w_{0}^{2} \kappa^{2}}{b_{f}^{2}} M
$$

where

$$
M \equiv 1-\exp \left(-b_{f}^{2} / w_{0}^{2}\right)\left[I_{0}\left(b_{f}^{2} / w_{0}^{2}\right)+I_{1}\left(b_{f}^{2} / w_{0}^{2}\right)\right]
$$

and $\left\{I_{n}(x), n=0,1,2, \ldots\right\}$ are the modified Bessel functions. Therefore, we have

$$
\kappa=\frac{b_{f}}{w_{0} \sqrt{2 M}}
$$


so that the normalized mode is simply

$$
\Psi(r, z)=\frac{2 Z}{b_{f} \sqrt{\pi M}} \int_{0}^{b_{f} / w} \exp \left[-Z(r / w-x)^{2}\right] \exp (-2 Z r x / w) I_{0}(2 Z r x / w) x d x
$$

which is straightforward to numerically integrate, the function $\mathrm{e}^{-X} I_{0}(X),\{X \in \mathbb{C}, \Re(X)>0\}$ having a simply form. One sees that the intensity profile is flat at the waist, with sharp wings (depending on the parameter $w_{0}$ ), and that the propagated mode is also almost flat. The beam's intensity profile (see Figure 3) is similar to a flat bump with rather sharp edges, so that the beam was called "mesa" by the previously mentioned Caltech team. The same mode propagated over kilometer-long distances exhibits a very weak distortion of its intensity profile despite diffraction. In foregoing numerical examples, we shall assume a symmetric cavity having a pair of identical mirrors matched to that kind of mode. The wavefront at $1.5 \mathrm{~km}$ from the waist in a $3 \mathrm{~km} \mathrm{long}$ cavity determines the mirror's shape (see Figure 4). This particular construction scheme gives a nearly flat mirror, apart from a small departure. This kind of mirror has been tested for the issues of angular alignment requirements and not found satisfactory [35]; this is why a new version has been proposed starting from spherical wave fronts in a nearly concentric cavity geometry. There is a duality relation, found by Savov et al. [35], which allows one to map the properties of this kind of beam to that of a "mesa" beam. In particular the intensity profile is identical on the mirror coating, so that the analysis we propose here is valid for the mesa beam model presented above and for the "nearly concentric cavity mode" as well (see Equation (16) in [3]).

We choose the parameters $w_{0}$ and $b$ in order to have $1 \mathrm{ppm}$ clipping losses. It is possible to reduce clipping losses either by a smaller $w_{0}$ or by a smaller $b$. However, reducing $w_{0}$ too much leads to distorted wavefronts and unfeasible mirrors. We have found a possible compromise with $w_{0}=3.2 \mathrm{~cm}$ and $b_{f}=10.7 \mathrm{~cm}$, giving exactly 1 ppm clipping losses on both $35 \mathrm{~cm}$ diameter mirrors.

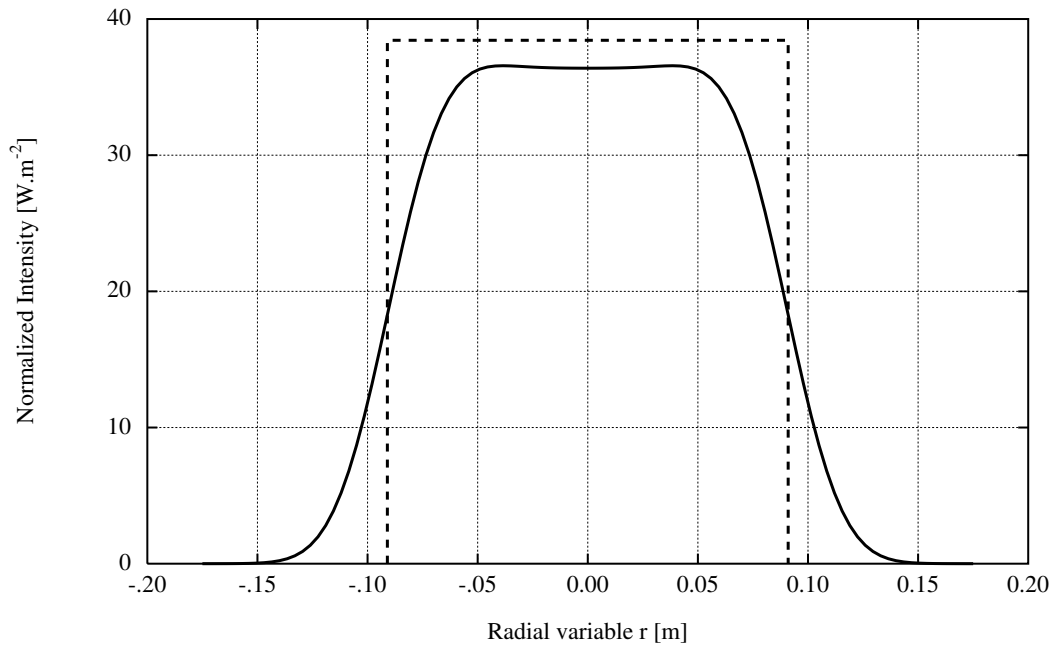

Figure 3: Solid line: Intensity profile of a normalized mesa mode of parameters $b_{f}=10.7 \mathrm{~cm}, w_{0}=3.2 \mathrm{~cm}$. Dashed line: nearest flat beam profile $(b=9.1 \mathrm{~cm})$.

For most of our purposes regarding thermal problems, a crude model (flat), reduced to the 


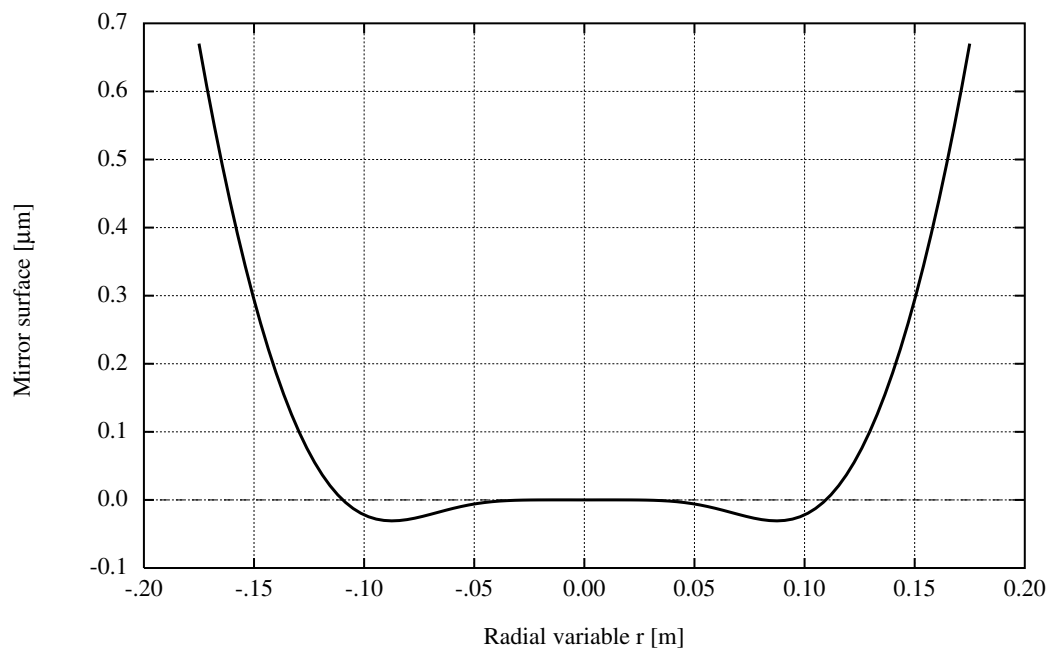

Figure 4: Surface of a mirror matching the mesa beam of parameters $b_{f}=10.7 \mathrm{~cm}, w_{0}=3.2 \mathrm{~cm}$

characteristic function of the disk, namely an intensity function of the form

$$
I(r)=\frac{1}{\pi b^{2}}\left\{\begin{array}{ll}
1 & (r \leq b) \\
0 & (r>b)
\end{array},\right.
$$

is sufficient. However, if need be, we will check that the conclusions drawn from the crude model (flat) are still valid for the realistic model (mesa), defined by Equation (2.16). However, in some specific cases (e.g., thermodynamical noise), the crude model leads to mathematical problems and cannot be used at all; thus, we must work with Equation (2.16). The value of $b$ is chosen such that the flat beam gives the darkest fringe when interfering with the mesa beam (minimizing the Hermitian distance). This gives, for the mesa beam described above, an effective value $b=9.1 \mathrm{~cm}$ (see Figure 3).

Throughout the following discussions, we shall numerically treat three examples. The first, "Ex1", is the current situation for the Virgo input mirrors, i.e., an $\mathrm{LG}_{0,0}$ mode of $w=2 \mathrm{~cm}$. The second, "Ex2", is the flat mode described above of $b=9.1 \mathrm{~cm}$, or, when needed, the mesa mode with $b_{f}=10.7 \mathrm{~cm}$ (1 ppm clipping loss). The third, "Ex3", is the $\mathrm{LG}_{5,5}$ mode of $w=3.5 \mathrm{~cm}$ (1 ppm clipping loss). However, the analytic expressions are general.

\subsection{Other exotic modes}

Several other types of modes have been or could be proposed in the same spirit of reducing the Brownian thermal noise and/or the thermoelastic noise.

\subsubsection{Bessel beams}

The search for weakly diffracting beams leads naturally to nondiffractive beams. There is an obvious solution to Helmholtz's equation in cylindrical coordinates;

$$
\psi_{n}(r, \varphi, z)=\exp (i \beta z) J_{n}(\alpha r) \exp (i n \varphi)
$$


where $n$ is an arbitrary integer, $J_{n}(x)$ a Bessel function, and $\alpha$ and $\beta$ are numbers such that $\alpha^{2}+\beta^{2}=k^{2}(k \equiv 2 \pi / \lambda)$. This was first noted (in the case $\left.n=0\right)$ by Durnin [16] and is thus called a "Durnin" beam by some. We feel that for clarity it is more appropriate to call it the "Bessel" beam. The case of $n=0$ is particularly interesting in the context of hyper-resolution, for instance, due to the sharp central peak when $\alpha$ is large, but it is forbidden in our case for the same reason. The transverse structure of such a wave is independent on $z$ and similar to a wave guided in the core of a cylindrical fiber (in the cladding, there is a different solution smoothly matched and of finite extension). However, the energy carried by a Bessel beam is infinite, exactly as in the case of a plane wave. In fact, the wavefront is flat in the case of $n=0$. The impossibility of generating waves of infinite extension leads to truncated waves having diffractive behavior and consequent clipping losses. The result depends on the method of truncation. The wavefront of such a truncated Bessel wave after propagation is hardly compatible with a reasonable mirror shape anyway.

\subsubsection{Conical-mirror or Gauss-Bessel beams}

The best way of truncating a Bessel beam is to make the following construction;

$$
\Psi_{n}(x, y, 0)=\frac{1}{2 \pi} \int_{0}^{2 \pi} \phi(x, y, 0) \mathrm{e}^{i k \theta(x \cos \psi+y \sin \psi)} d \psi,
$$

where $\phi(x, y, z)$ refers to a $\mathrm{TEM}_{00}$ mode of width parameter $w_{0}$. In words, we add elementary Gaussian waves whose propagation axes generate a cone of small aperture $\theta$, having its axis along the $z$ direction and its vertex at $z=0$. We assume that this is the situation at the middle of a $3 \mathrm{~km}$ cavity, and are interested in the amplitude at the end (or input) mirror. A sum of elementary Gaussian beams may be propagated by propagating each component separately and summing up at the end. We get, up to some phase factors irrelevant for expressing the intensity, the following amplitude for the mode at any abscissa $z$;

$$
\Psi_{n}(r, \varphi, z)=\kappa \exp \left[-\frac{Z\left(r^{2}+\theta^{2} z^{2}\right)}{w(z)^{2}}\right] J_{0}\left(\frac{k r \theta}{\bar{Z}}\right),
$$

where $z_{R} \equiv \pi w_{0}^{2} / \lambda$ and $Z \equiv 1-i z / z_{R}$ (as above). $\kappa$ is a normalization factor. We have the standard relation $w(z)=w_{0} \sqrt{Z \bar{Z}}$. This formula makes it clear that this solution is a Bessel mode truncated by a Gaussian envelope. We therefore call these "Gauss-Bessel" modes. The intensity pattern of such modes depend obviously on the width parameter $w_{0}$ and on the aperture angle $\theta$. Combinations of these exist such that the intensity pattern is spread on the mirror surface, apart from a central peak. An example is shown in Figure 5. The wavefront is nearly conical (see Figure 6).

Bondarescu et al. [4] have carried out an optimization of coating thermal noise by combining LG modes. Using the better series of coefficients, they reach a wave analogous to a Gauss-Bessel mode and with a conical wavefront of the same kind. We intend to include these kinds of modes in an update to this review. To be specific, we give, in the section related to coating thermal noise (8.3.2), the figure of merit of the mode described in Figures 5 and 6 , which is not optimal, but already exhibits a good value, regarding coating thermal noise in the infinite mirror approximation (see Section 8.3). 


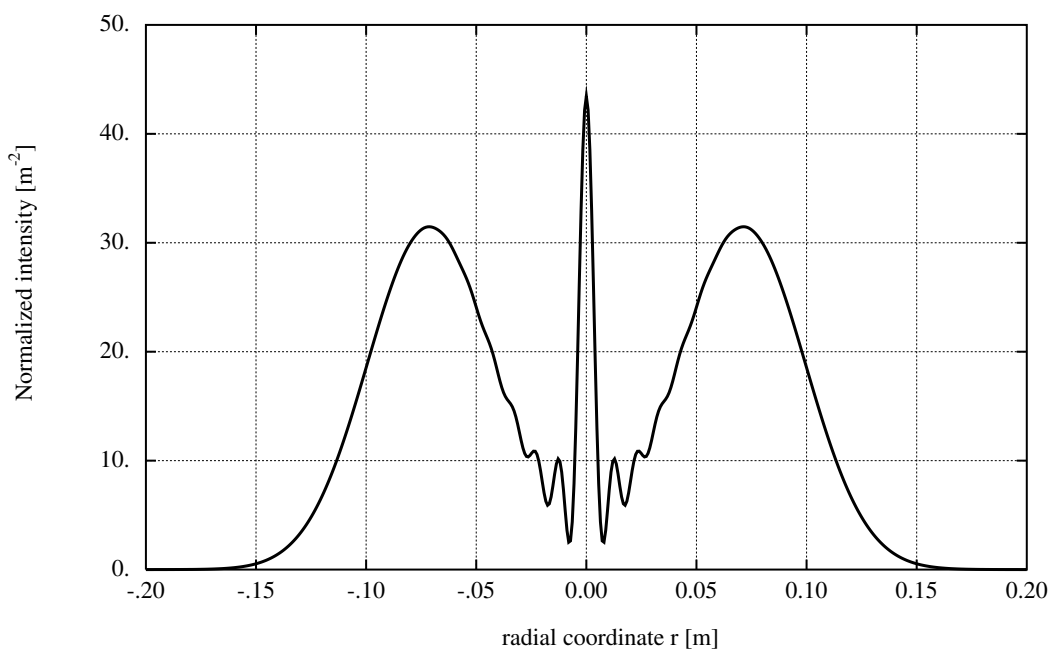

Figure 5: Power distribution of a Gauss-Bessel mode of parameters $\theta=54 \mu \mathrm{Rd}, w_{0}=5.2 \mathrm{~cm}, z=1.5 \mathrm{~km}$

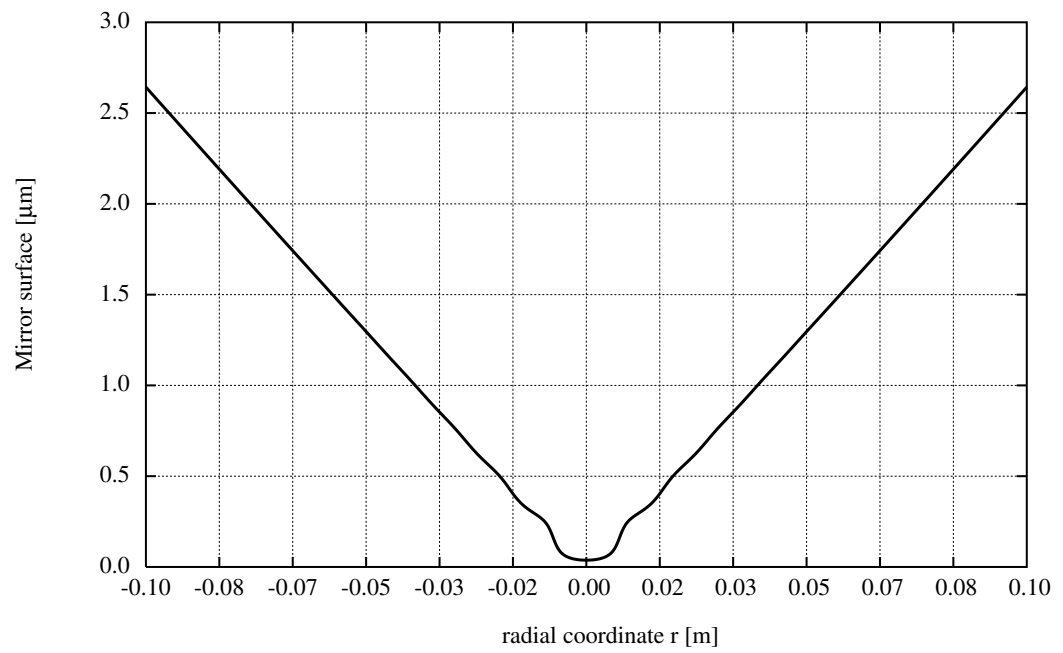

Figure 6: Surface of a mirror matching a Gauss-Bessel mode of parameters $\theta=54 \mu \mathrm{Rd}, w_{0}=5.2 \mathrm{~cm}$, $z=1.5 \mathrm{~km}$ 


\section{$3 \quad$ Heating and Thermal Effects in the Steady State}

We consider here the direct effects resulting from absorption of light in the mirrors (either on the reflecting surface or in the bulk material) and the indirect effects, such as thermal lensing and thermal distortions. First, we discuss the steady state (the principles are given in [20]), then the quasi-static case (heating from an initial uniform temperature [21]), and finally the general dynamical case. We consider the case of cavity mirrors storing large optical power, heated partially by thermalization of light at the coated face. There is also heating by propagation losses inside the substrate. We assume thermal equilibrium by thermal radiation; the mirror being suspended by thin wires in a vacuum, there is no convection loss and we neglect conduction loss. We further assume a small relative excess of temperature, justified by the good quality of the coatings and of the bulk silica. With these assumptions, the problem becomes linear and we can treat separately the contribution to heating caused by the coating and the bulk substrate. We consider a cylindrical mirror of diameter $2 a$ and of thickness $h$ (see Figure 7). The coordinates are radial $0 \leq r \leq a$, azimuthal $0 \leq \varphi \leq 2 \pi$ and longitudinal $-h / 2 \leq z \leq h / 2$.

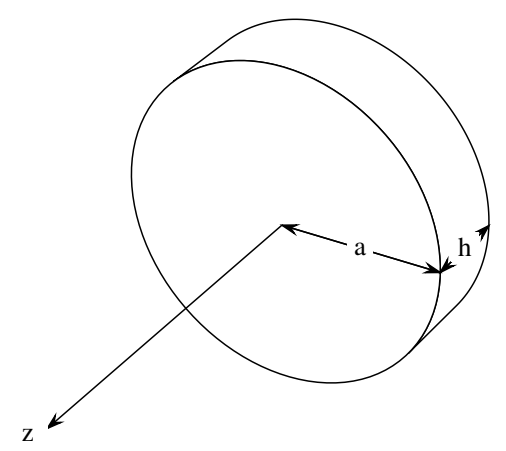

Figure 7: Notations for a cylindrical mirror

\subsection{Steady temperature field}

\subsubsection{Coating absorption}

Let us briefly recall that in the steady state with no internal source of heat, the Heat (Fourier) equation reads:

$$
\left(\partial_{r}^{2}+\frac{1}{r} \partial_{r}+\frac{1}{r^{2}} \partial_{\varphi}^{2}+\partial_{z}^{2}\right) T(r, \varphi, z)=0
$$

so that the temperature field is a harmonic function. A harmonic function is, for example,

$$
T_{n}(r, \varphi, z)=\mathrm{e}^{ \pm k z} J_{n}(k r) \mathrm{e}^{i n \varphi}
$$

where $\left\{J_{n}(x), n=0,1, ..\right\}$ are Bessel functions of the first kind, $n$ is an arbitrary integer and $k$ an arbitrary constant. We assume the readout beam reflected by the coating has an intensity distribution of the form

$$
I(r, \phi)=I_{n}(r) \cos \left(n \varphi+\varphi_{n}\right)
$$


( $\varphi_{n}$ is an arbitrary constant) and thus having a simple angular parity (possibly one term of a Fourier series). Therefore, we define the temperature field as

$$
T_{n}(r, \varphi, z)=\mathrm{e}^{ \pm k z} J_{n}(k r) \cos \left(n \varphi+\varphi_{n}\right) .
$$

The boundary conditions describe the heat flows on the faces and on the edge. The powerful light beam is assumed to be reflected at $z=-h / 2$. If $K$ denotes the thermal conductivity and $T_{0}$ the (homogeneous) temperature of the surrounding walls of the vacuum vessel, and if we consider $T_{n}(r, \varphi, z)$ as the excess of temperature with respect to $T_{0}$, we have, at thermal equilibrium, the following balance on the irradiated face;

$$
-K\left[\frac{\partial T_{n}(r, \varphi, z)}{\partial z}\right]_{z=-h / 2}=-\sigma_{\mathrm{f}}\left(\left[T_{0}+T_{n}(r, \varphi,-h / 2)\right]^{4}-T_{0}^{4}\right)+\epsilon I_{n}(r) \cos \left(n \varphi+\varphi_{n}\right),
$$

where the left-hand side represents the power lost by the substrate and the first term of the right-hand side represents the power flow of thermal radiation according to Stefan's law. $\sigma_{\mathrm{f}}$ is the Stefan-Boltzmann (SB) constant $\left(\sigma_{\mathrm{f}} \sim 5.6710^{-8} \mathrm{~W} \mathrm{~m}^{-1} \mathrm{~K}^{-4}\right)$ corrected for the emissivity of silica; accounting for a possible special processing of the edge (e.g., some thin metallic layer), we shall allow different values of the $\mathrm{SB}$ constant on the faces, $\sigma_{\mathrm{f}}$, and on the edge, $\sigma_{\text {edge. }}$ The second term of the right-hand side is the density of power received from the light beam. $\epsilon$ represents the relative loss at reflection. After linearization (i.e., assuming $T_{n} \ll T_{0}$ ), we get

$$
-K\left[\frac{\partial T_{n}(r, \varphi, z)}{\partial z}\right]_{z=-h / 2}=-4 \sigma_{\mathrm{f}} T_{0}^{3} T_{n}(r, \varphi,-h / 2)+\epsilon I_{n}(r, \varphi) .
$$

On the opposite face, we have a similar condition (the radiation flow is in the opposite direction)

$$
-K\left[\frac{\partial T_{n}(r, \varphi, z)}{\partial z}\right]_{z=h / 2}=4 \sigma_{\mathrm{f}} T_{0}^{3} T_{n}(r, \varphi, h / 2),
$$

whereas on the edge, the boundary condition is

$$
-K\left[\frac{\partial T_{n}(r, \varphi, z)}{\partial r}\right]_{r=a}=4 \sigma_{\text {edge }} T_{0}^{3} T_{n}(r, \varphi, a) .
$$

This last condition is relative to the radial function and gives

$$
-K k J_{n}^{\prime}(k a)=4 \sigma_{\text {edge }} T_{0}^{3} J_{n}(k a)
$$

or, by introducing the reduced radiation constant $\chi_{e} \equiv 4 \sigma_{\text {edge }} T_{0}^{3} a / K$,

$$
k a J_{n}^{\prime}(k a)+\chi_{e} J_{n}(k a)=0 .
$$

An equation of the form

$$
\zeta J_{n}^{\prime}(\zeta)+\chi_{e} J_{n}(\zeta)=0
$$

has an infinite and discrete family of solutions $\left\{\zeta_{n, s}, s=1,2, \ldots\right\}$. Therefore, a sufficiently general solution of the Heat equation having a given angular parity will be taken as a series,

$$
T_{n}(r, \varphi, z)=\sum_{s>0} T_{n, s}(z) J_{n}\left(\zeta_{n, s} r / a\right) \cos \left(n \varphi+\varphi_{n}\right)
$$

Moreover, after the Sturm-Liouville theorem, the family of functions $\left\{J_{n}\left(\zeta_{n, s} r / a\right), s=1,2, \ldots\right\}$ is orthogonal and complete on the interval $[0, a]$. The normalization factor is (see, for instance, Equation (11.4.5) in [1])

$$
\int_{0}^{1} x J_{n}\left(\zeta_{p, s} x\right) J_{n}\left(\zeta_{p, s^{\prime}} x\right) d x=\delta_{s, s^{\prime}} \frac{1}{2 \zeta_{n, s}^{2}}\left(\chi_{e}^{2}+\zeta_{n, s}^{2}-n^{2}\right) J_{n}\left(\zeta_{n, s}\right)^{2} .
$$


The first consequence is that it is possible to express the radial intensity function $I_{n}(r)$ of integrated power $P$ in the form of a Fourier-Bessel (FB) series,

$$
I_{n}(r)=\frac{P}{\pi a^{2}} \sum_{s>0} p_{n, s} J_{n}\left(\zeta_{n, s} r / a\right) .
$$

The dimensionless FB coefficients $\left\{p_{n, s}, s=1,2, \ldots\right\}$ being obtained by

$$
p_{n, s}=\frac{2 \pi \zeta_{n, s}^{2}}{P\left(\chi_{e}^{2}+\zeta_{n, s}^{2}-n^{2}\right) J_{n}\left(\zeta_{n, s}\right)^{2}} \int_{0}^{a} I_{n}(r) J_{n}\left(\zeta_{n, s} r / a\right) r d r .
$$

Now the longitudinal function is of the form

$$
T_{n, s}(z)=A_{n, s} \exp \left(\zeta_{n, s} z / a\right)+B_{n, s} \exp \left(-\zeta_{n, s} z / a\right)
$$

and the constants $A_{n, s}$ and $B_{n, s}$ are determined by the boundary conditions (3.6) and (3.7). Finally, one finds

$$
T_{n, s}(z)=\frac{\epsilon P}{\pi K a} p_{n, s} \mathrm{e}^{-\zeta_{n, s} h / 2 a} \frac{\left(\zeta_{n, s}-\chi\right) \mathrm{e}^{-\zeta_{n, s}(h-z) / a}+\left(\zeta_{n, s}+\chi\right) \mathrm{e}^{-\zeta_{n, s} z / a}}{\left(\zeta_{n, s}+\chi\right)^{2}-\left(\zeta_{n, s}-\chi\right)^{2} \mathrm{e}^{-2 \zeta_{n, s} h / a}},
$$

where $\chi$ is the reduced radiation constant for faces (i.e., $\left.\chi \equiv 4 \sigma_{\mathrm{f}} T_{0}^{3} a / K\right)$. This completely determines the temperature field through Equation (3.12), once the zeroes $\zeta_{n, s}$ are known by solving Equation (3.11), and once the coefficients $p_{n, s}$ are computed by the integration (3.15). This last point will be treated in Section 3.1.3 below. We can write Equation (3.17) in a more compact form exhibiting the symmetric and antisymmetric parts,

$$
T_{n, s}(z)=\frac{\epsilon P}{2 \pi K a} p_{n, s}\left[\frac{\cosh \left(\zeta_{n, s} z / a\right)}{d_{1, n, s}}-\frac{\sinh \left(\zeta_{n, s} z / a\right)}{d_{2, n, s}}\right]
$$

with the following definitions (used in all parts of this review),

$$
\left\{\begin{array}{l}
d_{1, n, s}=\zeta_{n, s} \sinh \gamma_{n, s}+\chi \cosh \gamma_{n, s} \\
d_{2, n, s}=\zeta_{n, s} \cosh \gamma_{n, s}+\chi \sinh \gamma_{n, s}
\end{array}\right.
$$

where $\gamma_{n, s} \equiv \zeta_{n, s} h / 2 a$. Note that if the heat source is located on the opposite face of the mirror, as in the case (to be treated later) of a thermal compensation beam, the preceding formula becomes simply

$$
T_{n, s}(z)=\frac{\epsilon P}{2 \pi K a} p_{n, s}\left[\frac{\cosh \left(\zeta_{n, s} z / a\right)}{d_{1, n, s}}+\frac{\sinh \left(\zeta_{n, s} z / a\right)}{d_{2, n, s}}\right] .
$$

\subsubsection{Bulk absorption}

Let us now assume that the heat source results from the loss of optical power by the beam inside the mirror substrate due to weak absorption. The beam intensity propagating inside the substrate is, strictly speaking, of the form

$$
I(r, z)=I(r) \exp [-\beta(z+h / 2)],
$$

where $I(r)$ is the incoming intensity. However, the linear absorption $\beta$ is assumed to be so weak that there is no significant change in amplitude of the beam along the optical path. Thus the heat source in the bulk material is $\beta I(r)\left(\mathrm{Wm}^{-3}\right)$. For any given angular parity, the Heat equation becomes

$$
\left(\partial_{r}^{2}+\frac{1}{r} \partial_{r}+\frac{1}{r^{2}} \partial_{\varphi}^{2}+\partial_{z}^{2}\right) T(r, \varphi, z)=-\frac{\beta}{K} I_{n}(r) \cos \left(n \varphi+\varphi_{n}\right) .
$$


We will look for a solution of the same form as Equation (3.12). The boundary condition on the edge is identical to Equation (3.8), so that the family of orthogonal functions is unchanged. The coefficients $p_{n, s}$ allowing expansion of the intensity function are also identical. Now we shall express the relevant solution as the sum of a special solution of Equation (3.22) and a more general solution of the homogeneous equation (identical to Equation (3.1)). Using the Bessel differential equation, the special solution of Equation (3.22) is found with

$$
T_{n, 1}(r, \varphi)=\frac{\beta P}{\pi K} \sum_{s>0} \frac{p_{n, s}}{\zeta_{n, s}^{2}} J_{n}\left(\zeta_{n, s} r / a\right) \cos \left(n \varphi+\varphi_{n}\right) .
$$

The solution of the homogeneous equation will be symmetric in $z$, owing to the independence of the heat source in $z$,

$$
T_{n, 2}(r, \varphi)=\sum_{s>0} A_{n, s} \cosh \left(\zeta_{n, s} z / a\right) J_{n}\left(\zeta_{n, s} r / a\right) \cos \left(n \varphi+\varphi_{n}\right)
$$

The arbitrary constants $A_{n, s}$ are determined by the boundary condition (3.7. Boundary condition (3.6) disappears, being identical to the preceding, due to symmetry. One finally finds a series analogous to Equation (3.12), except that the longitudinal function $T_{n, s}(z)$ is now

$$
T_{n, s}(z)=\frac{\beta P}{\pi K} \frac{p_{n, s}}{\zeta_{n, s}^{2}}\left[1-\frac{\chi \cosh \left(\zeta_{n, s} z / a\right)}{d_{1, n, s}}\right] .
$$

\subsubsection{Fourier-Bessel expansion of the readout beam intensity}

We address now the central point of the calculation of the FB coefficients $p_{n, s}$ of the intensity. The $\mathrm{LG}_{m, n}$ mode of integrated power $P$ has the following intensity function

$$
I_{m}^{(n)}(r, \varphi)=2 \mu_{n} R_{m}^{(n)}(r) \cos \left(n \varphi+\varphi_{n}\right)^{2},
$$

with $\mu_{n} \equiv 1 /\left(1+\delta_{n, 0}\right)$, and

$$
R_{m}^{(n)}(r)=\frac{2 P}{\pi w^{2}} \frac{m !}{(n+m) !}\left(\frac{2 r^{2}}{w^{2}}\right)^{n} L_{m}^{(n)}\left(\frac{2 r^{2}}{w^{2}}\right)^{2} \exp \left(\frac{2 r^{2}}{w^{2}}\right),
$$

where the functions $L_{q}^{(p)}(X)$ are the generalized Laguerre polynomials. The function can be split into two terms of simple angular parity,

$$
I_{m}^{(n)}(r, \varphi)=\mu_{n} R_{m}^{(n)}(r)+\mu_{n} R_{m}^{(n)} \cos \left(2 n \varphi+2 \varphi_{n}\right),
$$

so that the FB series of the intensity will be two-fold,

$$
I_{m}^{(n)}(r, \varphi)=\sum_{s>0} p_{0, s} J_{0}\left(\zeta_{0, s} r / a\right)+\sum_{s>0} p_{n, s} J_{2 n}\left(\zeta_{n, s} r / a\right) \cos \left(2 n \varphi+2 \varphi_{n}\right)
$$

where $\left\{\zeta_{0, s}\right\}$ are all solutions of

$$
\zeta J_{0}^{\prime}(\zeta)+\chi_{e} J_{0}(\zeta)=0
$$

whereas $\left\{\zeta_{n, s}\right\}$ are all solutions of

$$
\zeta J_{2 n}^{\prime}(\zeta)+\chi_{e} J_{2 n}(\zeta)=0 .
$$

The $p_{0, s}$ are given by [see Equation (3.15)]

$$
p_{0, s}=\frac{2 \pi \zeta_{0, s}^{2}}{P\left(\chi_{e}^{2}+\zeta_{0, s}^{2}\right) J_{0}\left(\zeta_{0, s}\right)^{2}} \int_{0}^{a} R_{m}^{(n)}(r) J_{0}\left(\zeta_{0, s} r / a\right) r d r
$$


In fact, the intensity is necessarily negligible near the edge, so that the upper bound of the integral may be replaced by $+\infty$ without appreciably changing the result. The integral then becomes explicitly computable, and one finds

$$
p_{0, s}=\frac{\zeta_{0, s}^{2}}{\left(\chi_{e}^{2}+\zeta_{0, s}^{2}\right) J_{0}\left(\zeta_{0, s}\right)^{2}} \mathrm{e}^{-y_{0, s}} L_{m}\left(y_{0, s}\right) L_{n+m}\left(y_{0, s}\right),
$$

where

$$
y_{0, s} \equiv \frac{\zeta_{0, s}^{2} w^{2}}{8 a^{2}}
$$

and the functions $L_{N}(X)$ are the (ordinary) Laguerre polynomials. In the same way, the coefficients $p_{n, s}$ are obtained by

$$
p_{n, s}=\frac{2 \pi \zeta_{n, s}^{2}}{P\left(\chi_{e}^{2}+\zeta_{n, s}^{2}-4 n^{2}\right) J_{2 n}\left(\zeta_{n, s}\right)^{2}} \int_{0}^{a} R_{m}^{(n)}(r) J_{2 n}\left(\zeta_{2 n, s} r / a\right) r d r .
$$

We can again replace the upper bound by $+\infty$, which allows explicit calculation,

$$
p_{n, s}=\frac{\zeta_{n, s}^{2}}{\left(\chi_{e}^{2}+\zeta_{n, s}^{2}-4 n^{2}\right) J_{2 p}\left(\zeta_{p, s}\right)^{2}} \frac{m !}{(n+m) !} \mathrm{e}^{-y_{n, s}}\left(y_{n, s}\right)^{n} L_{m}^{(n)}\left(y_{n, s}\right)^{2}
$$

with the notation

$$
y_{n, s} \equiv \frac{\zeta_{n, s}^{2} w^{2}}{8 a^{2}} .
$$

In the latter case we see that the Hankel transform maps the square of a Laguerre-Gauss function onto the same function with a different argument, up to a scaling factor. The temperature field is now completely known. Let us add that the Fourier-Bessel series are rapidly convergent so that the reconstruction of the intensity is obtained with excellent accuracy with only 50 terms. In Figure 8 we show the difference between the original intensity and the reconstructed one.

In the case of an ideally flat mode of radius $b$, the integral (3.15) is trivial, and we have the following $\mathrm{FB}$ coefficients:

$$
p_{\mathrm{flat}, s}=\frac{2 a}{b} \frac{\zeta_{0, s}}{\left(\chi^{2}+\zeta_{0, s}^{2}\right) J_{0}^{2}\left(\zeta_{0, s}\right)} J_{1}\left(\zeta_{0, s} b / a\right) .
$$

The reconstruction of the flat mode from a limited number of Fourier-Bessel coefficients is not perfect, owing to the generation of high frequencies by the sharp edges. But the heat field itself is rapidly convergent because of the regularizing effect of integration, so that even with a small number of terms, the FB series are accurate. In cases where the ideally flat model is forbidden, the FB coefficients must be computed using Equation (2.16) via a numerical integration.

See Figure 9 for the reconstructed intensity profile of a Laguerre-Gauss mode $\mathrm{LG}_{5,5}$.

\subsubsection{Numerical results on temperature fields}

If we assume a mirror of the Virgo input mirrors size, made of synthetic silica, we can take the parameters of Table 1 .

A cut of the temperature field in the $\varphi=0$ plane can be seen in Figures 10, 12, and 14 for heating by coating absorption, and in Figures 11, 13, and 15 for heating by dissipation in the substrate.

The temperature map of the coating $z=-h / 2$ is shown in Figure 16 (heating by coating absorption). 


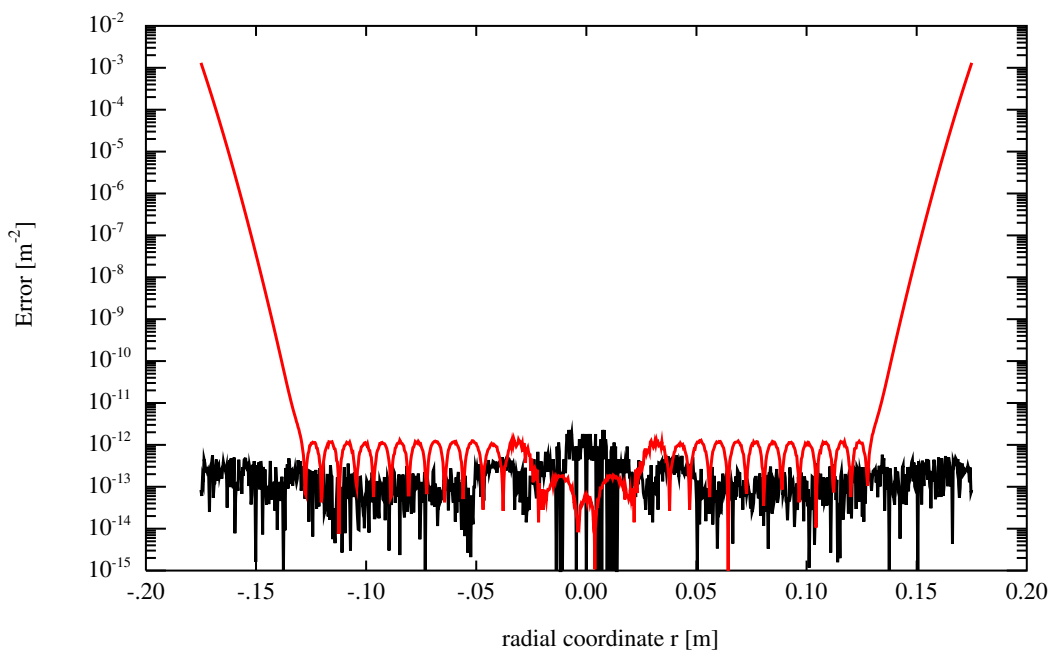

Figure 8: Error in intensity reconstruction (50 Fourier-Bessel terms) for $\mathrm{LG}_{0,0}, w=2 \mathrm{~cm}$ (black curve) and $\mathrm{LG}_{5,5}, w=3.5 \mathrm{~cm}$ (red curve); Cut: $\varphi=0$

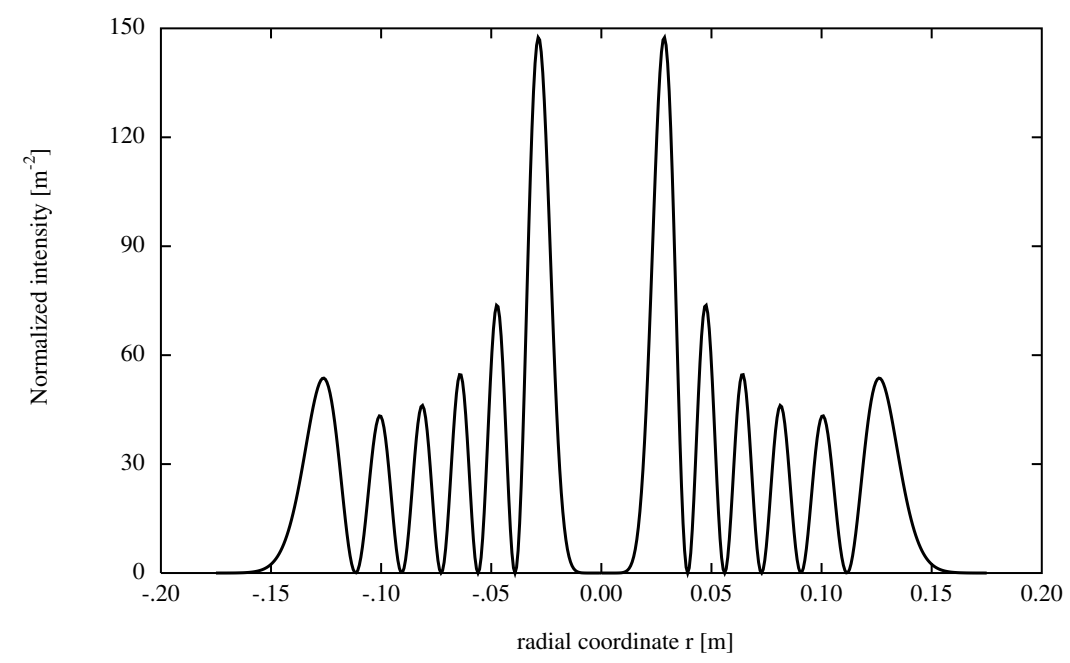

Figure 9: Reconstructed intensity (FB series) for $\mathrm{LG}_{5,5}$ mode $(\varphi=0)$ 
Table 1: Physical constants used in this paper

\begin{tabular}{llll}
\hline Symbol & Parameter & Value & units \\
\hline$a$ & half diameter & 0.175 & $\mathrm{~m}$ \\
$h$ & thickness & 0.1 & $\mathrm{~m}$ \\
$\rho$ & density & 2,202 & $\mathrm{~kg} \mathrm{~m}^{-3}$ \\
$K$ & thermal conductivity & 1.38 & $\mathrm{Wm}^{-1} \mathrm{~K}^{-1}$ \\
$C$ & specific heat cap. & 745 & $\mathrm{~J} \mathrm{~kg}^{-1} \mathrm{~K}^{-1}$ \\
$\alpha$ & thermal expansion coef. & $5.4 \times 10^{-7}$ & $\mathrm{~K}^{-1}$ \\
$\beta$ & linear absorption & $10^{-5}$ & $\mathrm{~m}^{-1}$ \\
$Y$ & Young's modulus & $7.3 \times 10^{10}$ & $\mathrm{Nm}^{-2}$ \\
$\sigma$ & Poisson ratio & 0.17 & dimensionless $_{d n / d T}$ \\
thermal refractive ind. & $1.1 \times 10^{-5}$ & $\mathrm{~K}^{-1}$ \\
\hline
\end{tabular}

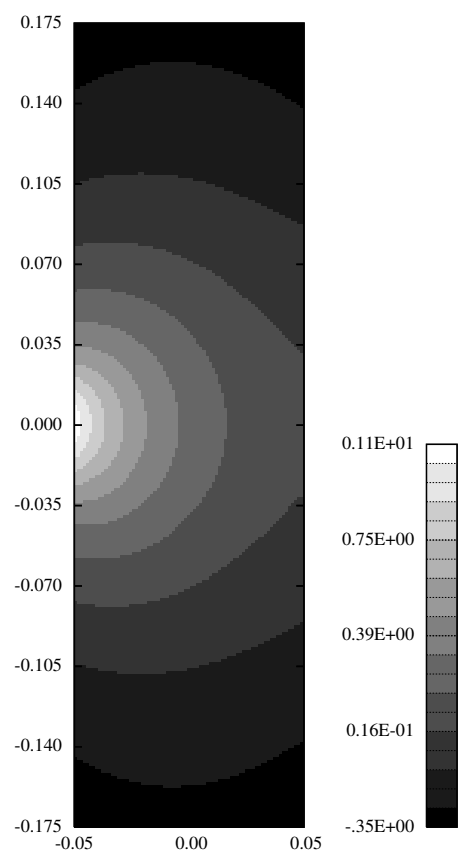

Figure 10: Temperature field in the substrate, $1 \mathrm{~W}$ dissipated in the coating, mode $\mathrm{LG}_{0,0}, w=2 \mathrm{~cm}$ $(\varphi=0)$ [logarithmic scale] 


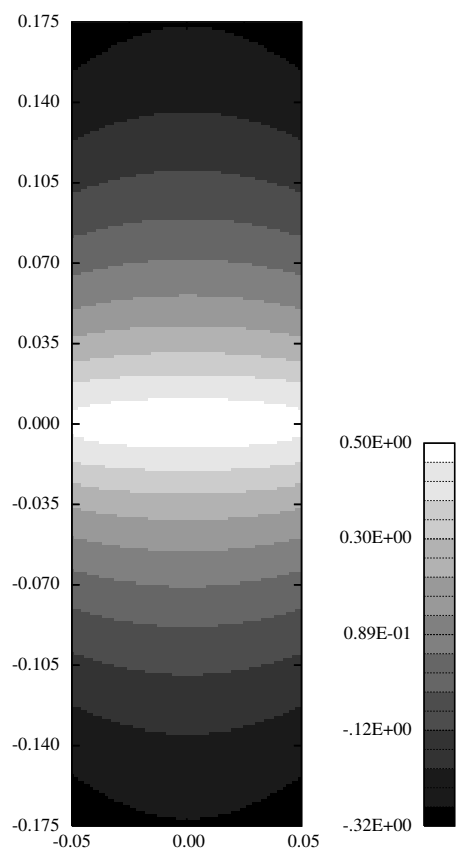

Figure 11: Temperature field in the substrate, $1 \mathrm{~W}$ dissipated in the bulk substrate, mode $\mathrm{LG}_{0,0}, w=2 \mathrm{~cm}$ $(\varphi=0)[\operatorname{logarithmic}$ scale] 


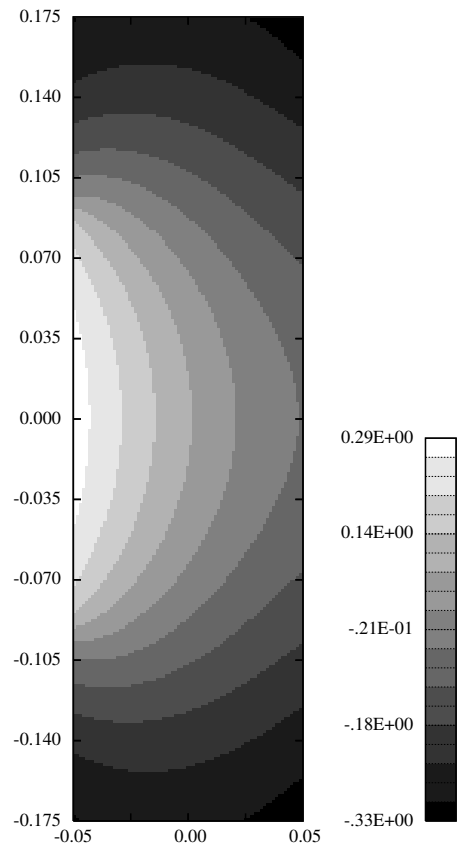

Figure 12: Temperature field in the substrate, $1 \mathrm{~W}$ dissipated in the coating, flat mode, $b=9.1 \mathrm{~cm}$ $(\varphi=0)$ [logarithmic scale] 


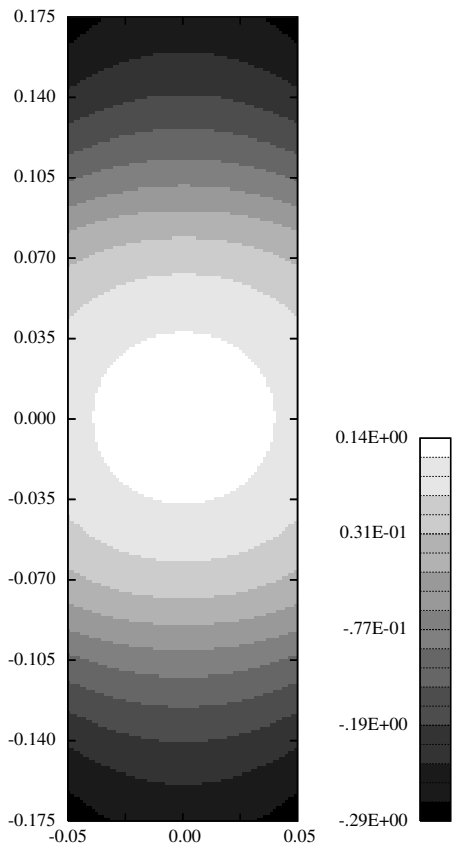

Figure 13: Temperature field in the substrate, $1 \mathrm{~W}$ dissipated in the bulk substrate, flat mode, $b=9.1 \mathrm{~cm}$ $(\varphi=0)$ [logarithmic scale] 


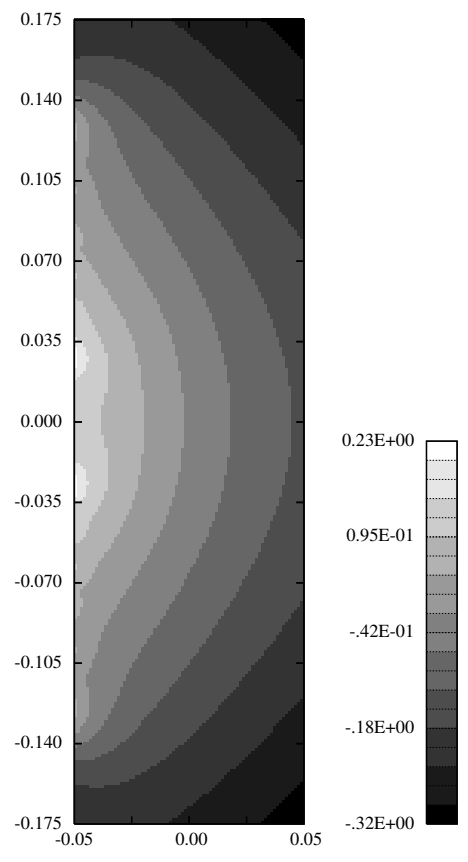

Figure 14: Temperature field in the substrate, $1 \mathrm{~W}$ dissipated in the coating, mode $\mathrm{LG}_{5,5}, w=3.5 \mathrm{~cm}$ $(\varphi=0)$ [logarithmic scale] 


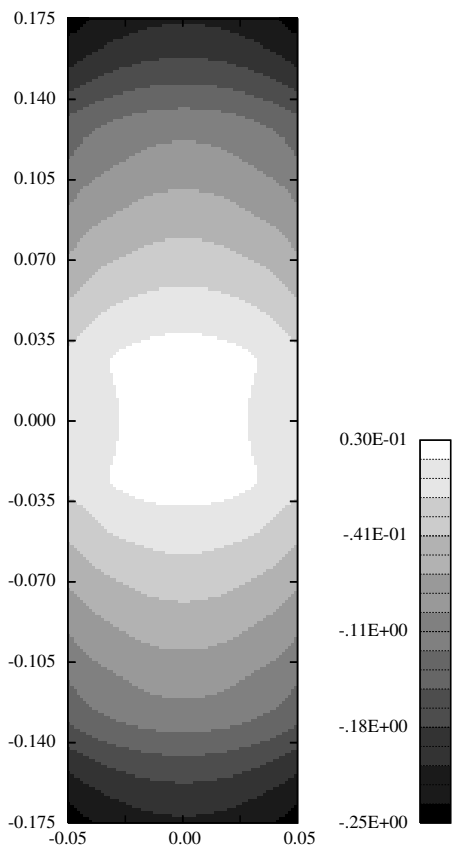

Figure 15: Temperature field in the substrate, $1 \mathrm{~W}$ dissipated in the bulk substrate, mode $\mathrm{LG}_{5,5}$, $w=3.5 \mathrm{~cm}(\varphi=0)$ [logarithmic scale] 


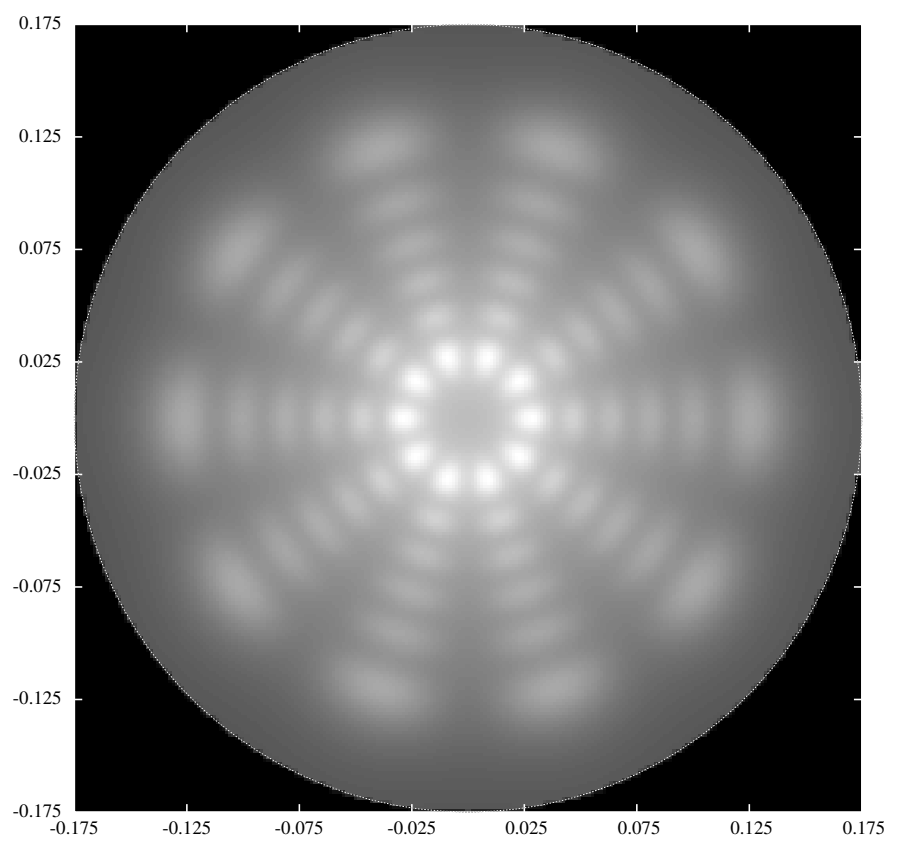

Figure 16: Nonaxisymmetric mode $\mathrm{LG}_{5,5}, w=3.5 \mathrm{~cm}$, temperature on the coating (coating absorption) $(z=-h / 2)$ 
The temperature map of the meridian plane $(z=0)$ of the substrate (heating by internal dissipation) is shown in Figure 17, where one can see the effect of thermal conduction, which generates a practically axisymmetric temperature field, despite the $\cos ^{2}$ signature of the incoming light beam.

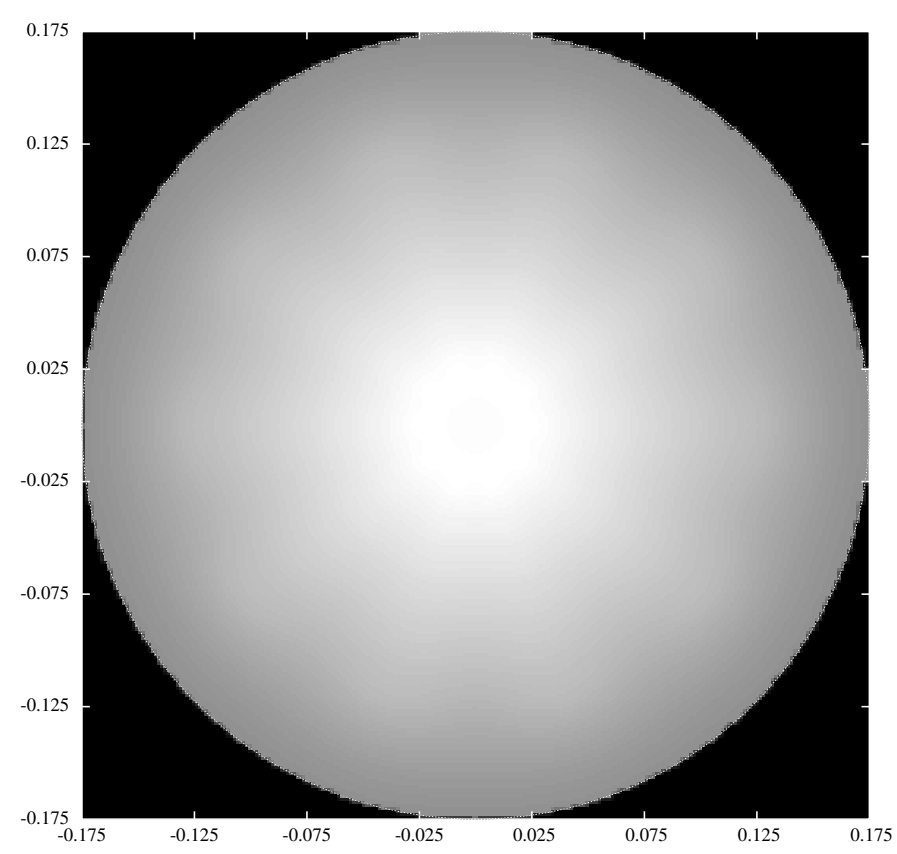

Figure 17: Nonaxisymmetric mode $\mathrm{LG}_{5,5}, w=3.5 \mathrm{~cm}$. Temperature in the meridian plane (bulk absorption) $(z=0)$

The dependence of the temperature field on the longitudinal variable $z$ is shown in Figures 18 and 19.

\subsection{Steady thermal lensing}

The temperature field inside the substrate has, as a first effect, to change the refractive index by an amount

$$
\delta n(r, z)=\frac{d n}{d T} T(r, z),
$$

where $d n / d T$ is the temperature index coefficient [23]. Therefore, the resulting index field, not being homogeneous, causes focusing effects on light called thermal lensing. The thermal lens is the integrated excess optical path (EOP) for a light ray crossing the substrate. Neglecting diffraction over the thickness of the mirror substrate, the EOP can be evaluated using

$$
Z(r)=\frac{d n}{d T} \int_{-h / 2}^{h / 2} T(r, z) d z
$$




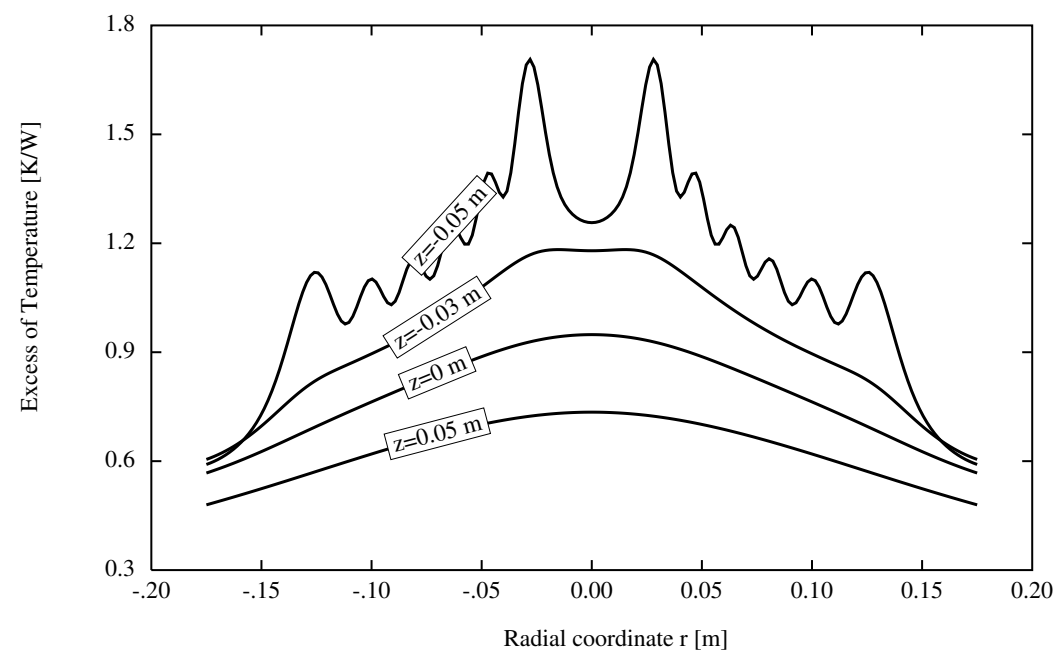

Figure 18: Temperature at various depths in the substrate (coating absorption) case of $\mathrm{LG}_{5,5}(\varphi=0)$

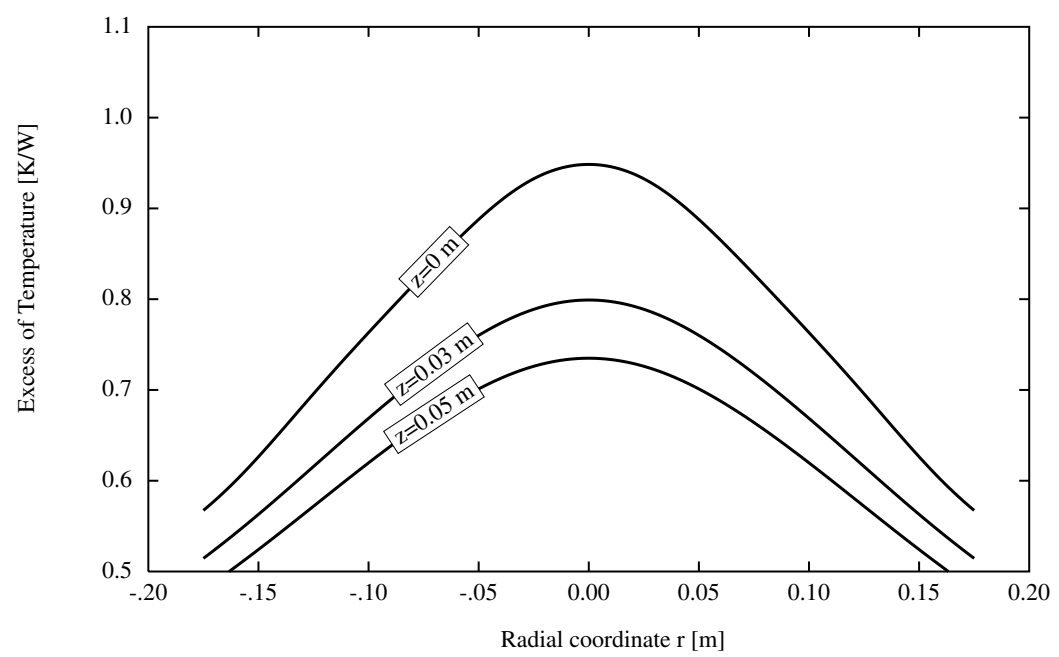

Figure 19: Temperature at various depths in the substrate (flat mode, bulk absorption) $(\varphi=0)$ 
It is easy to obtain the result in the two cases discussed above.

\subsubsection{Thermal lensing from coating absorption}

From Equation (3.18) we obtain the thermal lens caused by coating absorption:

$$
Z_{\text {coat }}(r)=\frac{d n}{d T} \frac{\epsilon P}{\pi K} \sum_{s} \frac{p_{n, s}}{\zeta_{n, s}} \frac{\sinh \gamma_{n, s}}{d_{1, n, s}} J_{n}\left(k_{n, s} r / a\right)
$$

\subsubsection{Thermal lens from bulk absorption}

From Equation (3.25), one finds

$$
Z_{\mathrm{bulk}}(r)=\frac{d n}{d T} \frac{\beta h P}{\pi K} \sum_{s} \frac{p_{s}}{\zeta_{n, s}^{2}}\left[1-\frac{\left(2 \chi a / \zeta_{n, s} h\right) \sinh \gamma_{s}}{d_{1, n, s}}\right] J_{n}\left(k_{n, s} r / a\right)
$$

\subsubsection{Equivalent paraboloid}

In order to study the consequences of the focusing properties of the thermal lens, we can compute the nearest paraboloid, defined by the apex equation :

$$
\hat{Z}(r)=c r^{2}+d,
$$

where $c$ is the curvature parameter, related to the mean curvature radius $R_{c}$ of the lens by $c=$ $1 / 2 R_{c} . d$ is called a piston. We define the nearest paraboloid by requiring the new lens $-\hat{Z}$ to carry out the best correction to the wavefront distorted by $Z$. If $\psi(r, \varphi)$ is the normalized mode amplitude, the Hermitian scalar product $S$ of the perfect incoming field with the distorted-corrected one is

$$
S=\langle\psi, C \psi\rangle
$$

where

$$
C(r, \phi)=\exp [i k(Z(r, \varphi)-\hat{Z}(r)] \quad(k \equiv 2 \pi / \lambda)
$$

( $\lambda$ being the laser wavelength). For a small difference, this is, at second order,

$$
S=1+i k \int_{\mathbb{R}^{2}}[Z(r, \varphi)-\hat{Z}(r)]|\psi(r, \varphi)|^{2} r d r d \varphi-\frac{k^{2}}{2} \int_{\mathbb{R}^{2}}[Z(r, \varphi)-\hat{Z}(r)]^{2}|\psi(r, \varphi)|^{2} r d r d \varphi .
$$

The first integral represents a phase that can always be cancelled out by a suitable choice of $d$. The second integral represents the coupling loss due to imperfect correction of the wavefront. Parameters $c$ and $d$ are found by requiring a minimum loss. If we define the average $<f>$ of any function $f(r, \varphi)$ by

$$
<f>=\int_{\mathbb{R}^{2}} f(r, \varphi)|\psi(r, \varphi)|^{2} r d r d \varphi
$$

In words, if all averages are performed with the weighting function $|\psi(r, \varphi)|^{2}$ (i.e., the normalized beam intensity), then the determination of the best paraboloid amounts to the classical leastsquares formulas:

$$
\begin{gathered}
c=\frac{<Z r^{2}>-<r^{2}><Z>}{<r^{4}>-<r^{2}>^{2}} \\
d=<Z>-c<r^{2}>.
\end{gathered}
$$




\subsubsection{Averaging with LG modes}

In the case of Laguerre-Gauss beams, the weighting function in the averaging process, is of the form (see Equation (3.27))

$$
|\psi(r, \varphi)|^{2}=R_{m}^{(n)}(r)+R_{m}^{(n)}(r) \cos \left(2 n \varphi+2 \varphi_{n}\right),
$$

whereas the thermal lens is of the form (see Equation (3.29)

$$
Z(r, \varphi)=Z_{0}(r)+Z_{n, m}(r) \cos \left(2 n \varphi+2 \varphi_{n}\right) .
$$

with

$$
\begin{gathered}
Z_{0}(r)=\sum_{s>0} Z_{0, s} J_{0}\left(\zeta_{0, s} r / a\right) \\
Z_{n, m}(r)=\sum_{s>0} Z_{n, m, s} J_{2 n}\left(\zeta_{n, s} r / a\right) .
\end{gathered}
$$

We have the following intermediate results:

$$
\begin{gathered}
<r^{2}>=2 \pi \int_{0}^{\infty} R_{m}^{(n)}(r) r^{3} d r=\frac{w^{2}}{2}(2 m+n+1) \\
<r^{4}>=2 \pi \int_{0}^{\infty} R_{m}^{(n)}(r) r^{5} d r=\frac{w^{4}}{4}[6 m(n+m+1)+(n+1)(n+2)]
\end{gathered}
$$

so that

$$
<r^{4}>-<r^{2}>^{2}=\frac{w^{4}}{4}[2 m(m+n+1)+n+1] .
$$

Now,

$$
<Z>=2 \pi \int_{0}^{\infty} R_{m}^{(n)}(r) Z_{0}(r) r d r+\pi \int_{0}^{\infty} R_{m}^{(n)}(r) Z_{n}(r) r d r
$$

making clear that we need the two integrals

$$
\begin{gathered}
\mathcal{I}_{0}=<J_{0}\left(\zeta_{0, s} r / a\right)>=2 \pi \int_{0}^{\infty} R_{m}^{(n)}(r) J_{0}\left(\zeta_{0, s} r / a\right) r d r \\
\mathcal{I}_{n, m}=<J_{2 n}\left(\zeta_{n, s} r / a\right)>=2 \pi \int_{0}^{\infty} R_{m}^{(n)}(r) J_{2 n}\left(\zeta_{n, s} r / a\right) r d r .
\end{gathered}
$$

These two integrals are analogous to those giving the FB coefficients of the intensity (see Equations (3.33) and (3.36)), so that we have immediately

$$
\begin{gathered}
\mathcal{I}_{0}=\mathrm{e}^{-y_{0, s}} L_{m}\left(y_{0, s}\right) L_{n+m}\left(y_{0, s}\right) \\
\mathcal{I}_{n, m}=\frac{m !}{(n+m) !}\left(y_{n, s}\right)^{n} \mathrm{e}^{-y_{n, s}} L_{m}^{(n)}\left(y_{n, s}\right)^{2} .
\end{gathered}
$$

To compute $<Z r^{2}>$, we need the integrals

$$
\begin{gathered}
\mathcal{K}_{0}=2 \pi \int_{0}^{\infty} R_{m}^{(n)}(r) J_{0}\left(\zeta_{0, s} r / a\right) r^{3} d r \\
\mathcal{K}_{n, m}=2 \pi \int_{0}^{\infty} R_{m}^{(n)}(r) J_{2 n}\left(\zeta_{n, s} r / a\right) r^{3} d r .
\end{gathered}
$$


These are easily obtained from the preceding definitions of $\mathcal{I}_{0}$, and $\mathcal{I}_{n, m}$ using the Bessel differential equation; namely, we have, for arbitrary $\kappa$,

$$
\begin{gathered}
<J_{0}(\kappa r) r^{2}>=-\left(\partial_{\kappa}^{2}+\frac{1}{\kappa} \partial_{\kappa}\right)<J_{0}(\kappa r)> \\
<J_{2 n}(\kappa r) r^{2}>=-\left(\partial_{\kappa}^{2}+\frac{1}{\kappa} \partial_{\kappa}-\frac{4 n^{2}}{\kappa^{2}}\right)<J_{2 n}(\kappa r)>.
\end{gathered}
$$

Let us note that

$$
\begin{aligned}
c_{0, s} & =\frac{<J_{0}\left(\zeta_{0, s} r / a\right) r^{2}>-<r^{2}><J_{0}\left(\zeta_{0, s} r / a\right)>}{<r^{4}>-<r^{2}>^{2}} \\
c_{n, m, s} & =\frac{<J_{2 n}\left(\zeta_{n, s} r / a\right) r^{2}>-<r^{2}><J_{2 n}\left(\zeta_{n, s} r / a\right)>}{<r^{4}>-<r^{2}>^{2}} .
\end{aligned}
$$

After some algebra, we get (here $y \equiv y_{0, s}$ )

$$
\begin{aligned}
c_{0, s}= & \frac{2}{[2 m(n+m+1)+n+1] w^{2}} \mathrm{e}^{-y}\left[(2 m+n+2-y) L_{m}(y) L_{n+m}(y)\right. \\
& +(m+1)\left(L_{m+1}(y) L_{n+m-1}^{(1)}(y)-L_{m}(y) L_{n+m}^{(1)}(y)\right) \\
& \left.+(n+m+1)\left(L_{m-1}(y) L_{n+m+1}^{(1)}(y)-L_{m}^{(1)}(y) L_{n+m}(y)\right)\right]
\end{aligned}
$$

and (here, $y \equiv y_{n, s}$ )

$$
\begin{aligned}
c_{n, m, s}= & \frac{2}{[2 m(n+m+1)+n+1] w^{2}} y^{n} \mathrm{e}^{-y} \\
& {\left[(2 n-y) L_{m}^{(n)}(y)^{2}+4(n+m-y) L_{m}^{(n)}(y) L_{m-1}^{(n+1)}(y)-\right.} \\
& \left.2(n+m)\left(L_{m}^{(n)}(y) L_{m-2}^{(n+1)}(y)+L_{m-1}^{(n+1)}(y) L_{m-1}^{(n)}(y)\right)\right]
\end{aligned}
$$

(with the convention $L_{m}^{(n)} \equiv 0[m<0]$ ). Now the curvature coefficient is simply

$$
c=\sum_{s>0}\left[c_{0, s} Z_{0, s}+\frac{1}{2} c_{n, m, s} Z_{n, m, s}\right] .
$$

3.2.3.2 Averaging with flat modes In the case of an ideally flat mode (crude model) of radius $b$, we get

$$
\begin{aligned}
\mathrm{V}\left[r^{2}\right] & =\frac{b^{4}}{12} \\
<J_{0}\left(\zeta_{0, s} r / a\right)> & =\frac{2 J_{1}\left(\zeta_{0, s} b / a\right)}{\zeta_{0, s} b / a}
\end{aligned}
$$

and

$$
\begin{aligned}
<J_{0}\left(\zeta_{0, s} r / a\right) r^{2}>-<r^{2}><J_{0}\left(\zeta_{0, s} r / a\right)>= & \frac{a^{2}}{\zeta_{s}^{2}}\left[4 J_{0}\left(\zeta_{s} b / a\right)+\right. \\
& \left.\left(\zeta_{s} b / a-8 a / b \zeta_{s}\right) J_{1}\left(\zeta_{s} b / a\right)\right] .
\end{aligned}
$$


The result for the curvature is

$$
c=\sum_{s>0} c_{s}^{(F)} Z_{0, s}
$$

with

$$
c_{s}^{(F)}=\frac{12 a^{2}}{b^{4} \zeta_{0, s}^{2}}\left[4 J_{0}\left(\frac{\zeta_{0, s} b}{a}\right)+\left(\frac{\zeta_{0, s} b}{a}-\frac{8 a}{\zeta_{0, s} b}\right) J_{1}\left(\frac{\zeta_{0, s} b}{a}\right)\right] .
$$

\subsubsection{Coupling losses}

The differences in thermal lensing between axisymmetric and nonaxisymmetric high-order beams are very small, because diffusion of heat rapidly produces a quasihomogeneous temperature with respect to the polar angle. This is why we now restrict the discussions to axisymmetric modes. Existence of a thermal lens $Z(r)$ causes a mismatching of the beam, which has passed through the lens, with the ideal one. The amplitude coupling coefficient is given by the Hermitian scalar product

$$
\left\langle\Psi, \mathrm{e}^{i k Z} \Psi\right\rangle=\left\langle\mathrm{e}^{i k Z}\right\rangle,
$$

where the average $\langle\ldots\rangle$ is weighted, as usual, by the normalized intensity of the readout beam. Mismatching results in turn in coupling losses due to the distortion of the wavefront of the transmitted beam. These distortions have been summarized by a spurious radius of curvature for which we have given formulas in the preceding paragraph (??). But it is clear from the shape of the lenses that the distortion is not parabolic. In general, there is some departure of the wavefront from a parabola. Thus, we have two contributions to coupling losses: a harmonic part (by reference to the harmonic oscillator potential) and a nonharmonic part. It is possible to compare the two contributions. For the parabolic or harmonic part, we have the following coupling coefficient $\gamma$ for a spurious curvature radius $R$ :

$$
\gamma=2 \pi \int_{0}^{a} \mathrm{e}^{i k r^{2} / 2 R}|\Psi(r)|^{2} r d r
$$

In the case of LG modes, we can be more specific:

$$
\gamma_{n, m}=\frac{m !}{(n+m) !} \frac{4}{w^{2}} \int_{0}^{a} \mathrm{e}^{i k r^{2} / 2 R}\left(2 r^{2} / w^{2}\right)^{n} L_{m}^{(n)}\left(2 r^{2} / w^{2}\right)^{2} \mathrm{e}^{-2 r^{2} / w^{2}} r d r .
$$

Assuming negligible clipping losses, we can replace the upper integration bound by $+\infty$ and take:

$$
\gamma_{n, m}=\frac{m !}{(n+m) !} \int_{0}^{\infty} x^{n} L_{m}^{(n)}(x)^{2} \mathrm{e}^{-(1-i F) x} d x
$$

(with $F \equiv \pi w^{2} / 2 \lambda R$ ) so that we obtain

$$
\gamma_{n, m}=\frac{1}{(1-i F)^{2 m+n+1}} \sum_{s=0}^{m}\left(\begin{array}{c}
m \\
s
\end{array}\right)\left(\begin{array}{c}
n+m \\
s
\end{array}\right)\left(-F^{2}\right)^{s} .
$$

In the case of a flat mode, the integral is trivial and we get:

$$
\left|\gamma_{\text {flat }}\right|^{2}=\operatorname{sinc}\left(\pi b^{2} / 2 \lambda R\right)^{2},
$$

while the power coupling losses are simply

$$
L=1-|\gamma|^{2} .
$$


In Figure 20, we have plotted the evolution of the coupling losses versus the dissipated power on the coating of a mirror. The solid and dashed curves correspond respectively to the total losses by a numerical integration of Equation (3.76) with the overall thermal lens and to harmonic losses. We see that all modes have almost only harmonic losses for weak dissipated losses (roughly below $100 \mathrm{~mW}$ ). The anharmonicity appears soon for the $\mathrm{LG}_{00}$ mode of width $2 \mathrm{~cm}$, and for a fraction of W, nonharmonic losses are significant. For Ex2 and Ex3, we see that the coupling losses are weaker and practically harmonic. The curve for $\mathrm{LG}_{55}$ is practically identical to the curve of the flat mode, which is why we did not plot it.

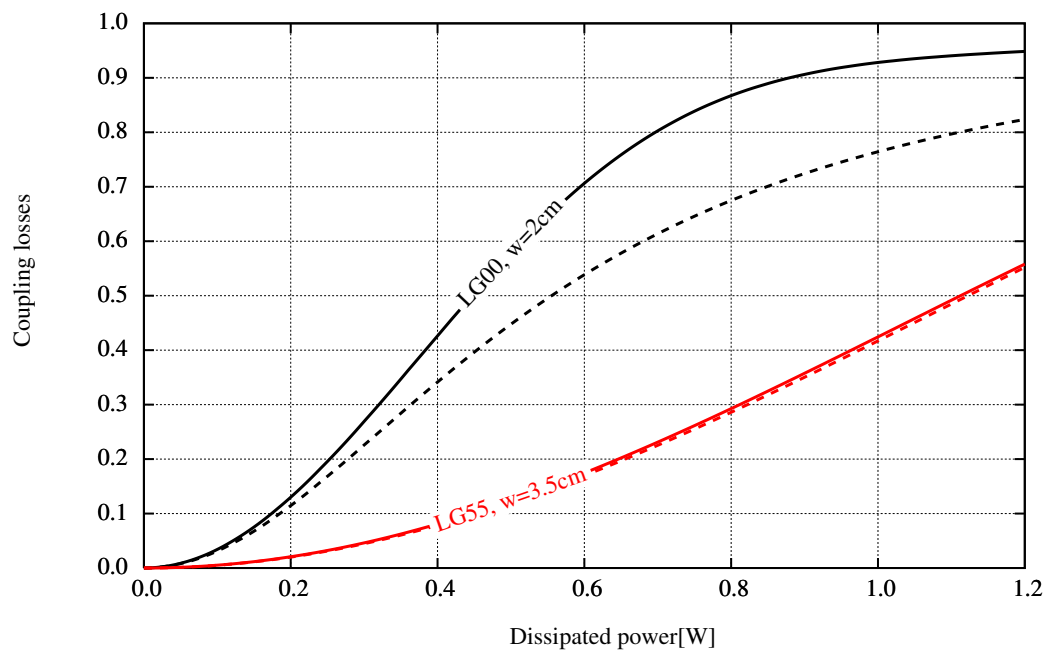

Figure 20: Coupling losses as functions of the dissipated power on the coating. Solid line: total losses, numerical integration of Equation (3.76). Dashed line: harmonic losses after Equation (3.80).

For weak dissipated power, in the case of LG modes we use the lowest-order approximation of Equation (3.80), so that we get

$$
L_{n, m}=[2 m(m+n+1)+n+1]\left(\frac{\pi w^{2}}{2 \lambda R}\right)^{2}+\mathcal{O}\left(R^{-4}\right) .
$$

We note that, owing to Equation (3.56), this is nothing but a variance of (weighted by the intensity profile)

$$
L_{n, m}=\left(\frac{\pi}{\lambda R}\right)^{2} V\left[r^{2}\right]=V\left[\frac{\pi r^{2}}{\lambda R}\right]
$$

Now for the flat mode, we get

$$
L_{\text {flat }}=\frac{1}{3}\left(\frac{\pi b^{2}}{2 \lambda R}\right)^{2}+\mathcal{O}\left(R^{-4}\right)
$$

which is again

$$
L_{\text {flat }}=V\left[\frac{\pi r^{2}}{\lambda R}\right] .
$$

NB: The curvature radii are expressed in $\mathrm{m} . \mathrm{W}$, the losses in $\mathrm{W}^{-2}$. 
Table 2: Thermal lensing from coating and bulk absorption (abs.)

\begin{tabular}{cccc}
\hline results (coating abs.) & $\mathrm{LG}_{0,0} w=2 \mathrm{~cm}$ & Flat $b=9.1 \mathrm{~cm}$ & $\mathrm{LG}_{5,5} w=3.5 \mathrm{~cm}$ \\
\hline curvature radius & $328 \mathrm{~mW}$ & $9,682 \mathrm{~mW}$ & $27,396 \mathrm{~mW}$ \\
piston & $3.23 \mu \mathrm{m} / \mathrm{W}$ & $1.43 \mu \mathrm{m} / \mathrm{W}$ & $1.08 \mu \mathrm{m} / \mathrm{W}$ \\
coupling losses & $3.24 / \mathrm{W}^{2}$ & $0.53 / \mathrm{W}^{2}$ & $0.51 / \mathrm{W}^{2}$ \\
\hline \hline results (bulk abs.) & $\mathrm{LG}_{0,0} w=2 \mathrm{~cm}$ & Flat $b=9.1 \mathrm{~cm}$ & $\mathrm{LG}_{5,5} w=3.5 \mathrm{~cm}$ \\
\hline curvature radius & $317 \mathrm{~mW}$ & $9,164 \mathrm{~mW}$ & $25,926 \mathrm{~mW}$ \\
piston & $3.39 \mu \mathrm{m} / \mathrm{W}$ & $1.52 \mu \mathrm{m} / \mathrm{W}$ & $1.15 \mu \mathrm{m} / \mathrm{W}$ \\
coupling losses & $3.47 / \mathrm{W}^{2}$ & $0.59 / \mathrm{W}^{2}$ & $0.56 / \mathrm{W}^{2}$ \\
\hline
\end{tabular}

\subsubsection{Numerical results}

The thermal lensing is almost identical for $1 \mathrm{~W}$ coating or bulk absorption. In Figure 21, we have plotted the thermal lens profile and the best fit paraboloid $\hat{Z}(r)$ for the case of heating by internal absorption.

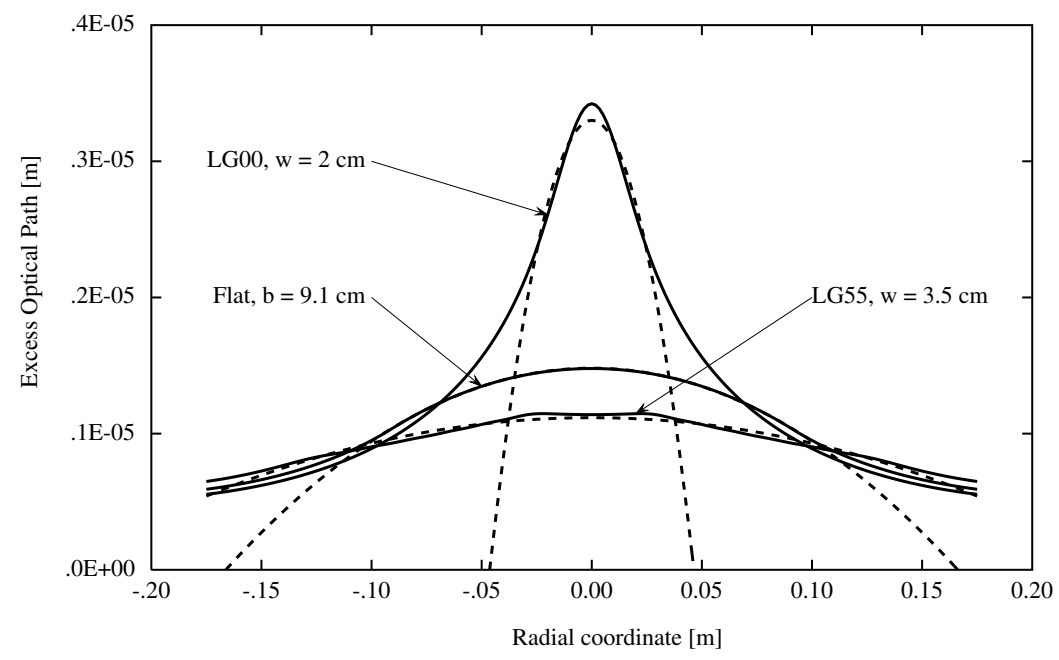

Figure 21: Thermal lens, heating by $1 \mathrm{~W}$ bulk absorption. The dashed line is the nearest paraboloid $\hat{Z}(r)$ (in the sense of least squares, weighted by the normalized beam intensity).

Some numerical results can be seen in Table 2. The losses are computed from the parabolic approximation [see Equations (3.83) and (3.85)] valid for weak dissipated power.

Table 3 contains results for some LG modes having $w$ parameters tuned for 1 ppm clipping losses on a $35 \mathrm{~cm}$ diameter mirror.

The difference between the flat beam and the mesa beam, regarding thermal lensing, is shown in Figure 22. One sees that using the crude flat beam yields a small overestimation of the lensing. 
Table 3: Thermal lensing curvature radii $\left(R_{c}\right)$ for LG modes having $1 \mathrm{ppm}$ clipping losses, and associated coupling losses (L) in the weak power approximation [Equation (3.83)] (mirror diameter: $35 \mathrm{~cm}$ )

\begin{tabular}{cccc||cc}
\hline order $(p, q)$ & $\mathrm{w}[\mathrm{cm}]$ & $R_{c}[\mathrm{~mW}]($ coat. abs. $)$ & $\mathrm{L}\left[\mathrm{W}^{-2}\right]$ & $R_{c}[\mathrm{~mW}]($ bulk abs. $)$ & $\left.\mathrm{L}^{-2}\right]$ \\
\hline$(0,0)$ & 6.65 & 4,400 & 2.20 & 4,184 & 2.43 \\
\hline$(0,1)$ & 5.56 & 8,566 & 1.42 & 8,139 & 1.57 \\
$(1,0)$ & 6.06 & 8,130 & 0.89 & 7,713 & 0.99 \\
\hline$(0,2)$ & 4.93 & 12,113 & 1.14 & 11,497 & 1.27 \\
$(1,1)$ & 5.23 & 11,608 & 0.97 & 11,006 & 1.08 \\
$(2,0)$ & 5.65 & 11,414 & 0.51 & 10,822 & 0.57 \\
\hline$(0,3)$ & 4.49 & 14,870 & 1.00 & 14,106 & 1.11 \\
$(1,2)$ & 4.70 & 14,430 & 0.92 & 13,677 & 1.02 \\
$(2,1)$ & 4.97 & 14,499 & 0.70 & 13,736 & 0.78 \\
$(3,0)$ & 5.35 & 14,484 & 0.34 & 13,729 & 0.38 \\
\hline$(0,4)$ & 4.17 & 17,219 & 0.91 & 16,328 & 1.01 \\
$(1,3)$ & 4.32 & 16,731 & 0.87 & 15,855 & 0.97 \\
$(2,2)$ & 4.52 & 16,889 & 0.73 & 15,997 & 0.82 \\
$(3,1)$ & 4.76 & 17,237 & 0.53 & 16,324 & 0.59 \\
$(4,0)$ & 5.11 & 17,368 & 0.25 & 16,462 & 0.27 \\
\hline$(0,5)$ & 3.91 & 19,117 & 0.85 & 18,123 & 0.95 \\
$(1,4)$ & 4.03 & 18,686 & 0.82 & 17,705 & 0.92 \\
$(2,3)$ & 4.18 & 18,787 & 0.74 & 17,792 & 0.82 \\
$(3,2)$ & 4.36 & 19,204 & 0.60 & 18,182 & 0.67 \\
$(4,1)$ & 4.58 & 19,790 & 0.42 & 18,738 & 0.46 \\
$(5,0)$ & 4.91 & 20,104 & 0.19 & 19,055 & 0.21 \\
\hline
\end{tabular}

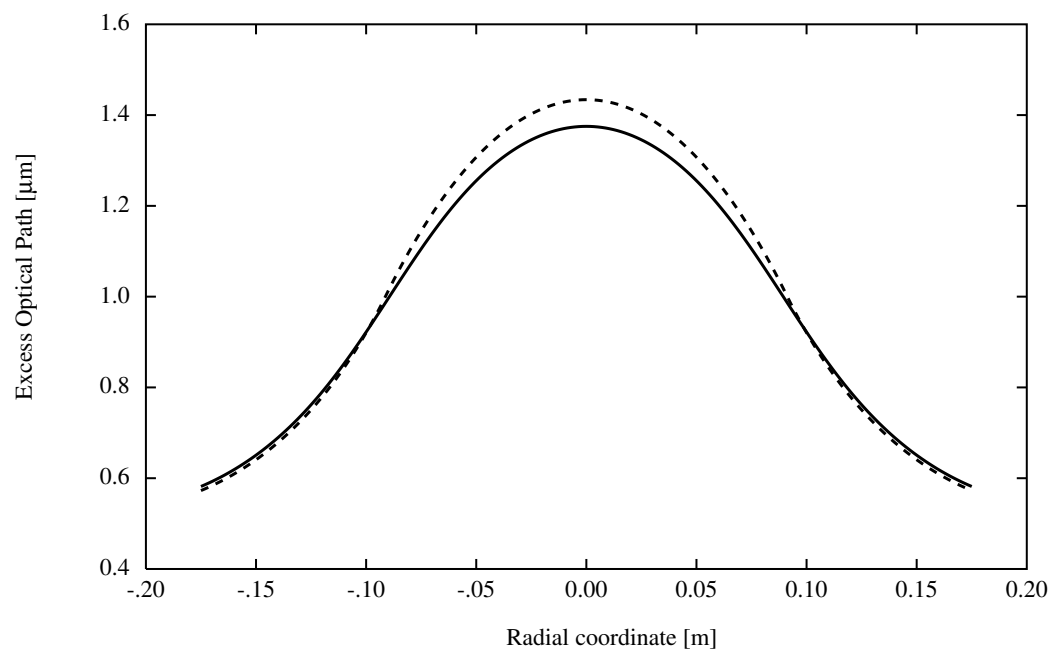

Figure 22: Thermal lens for $1 \mathrm{~W}$ absorbed from the mesa mode (solid line) and the flat mode (dashed line) 


\subsection{Thermal distortions in the steady state}

We assume from now on that the temperature field is axisymmetric. Due to the temperature field, at the same time, thermal expansion of the material causes a change of the shape of the mirror and a distortion of the reflecting face. If we call $\vec{u}(r, z)$ the displacement vector, i.e., the difference between the coordinates of a given atom before and after heating, the classical relevant quantities are the strain tensor $E_{i j}$ and the stress tensor $\Theta_{i j}$. In the presence of a temperature field $T$, the two tensors are related by the generalized Hooke law for isotropic media via the Lamé coefficients $\lambda, \mu$ :

$$
\Theta_{i j}=\delta_{i j}(\lambda E-\nu T)+2 \mu E_{i j}
$$

where $\nu$ is the stress temperature modulus and $E$ the trace of the strain tensor. $\delta_{i j}$ is the Kronecker tensor. In cylindrical coordinates $(r, \phi, z)$, assuming cylindrical symmetry, the displacement vector has only two components, $u_{r}(r, z)$ and $u_{z}(r, z)$. Then the strain tensor has four components:

$$
\begin{aligned}
& E_{r r}(r, z)=\frac{\partial u_{r}}{\partial r}(r, z), E_{\phi \phi}(r, z)=\frac{u_{r}(r, z)}{r} \\
& E_{z z}(r, z)=\frac{\partial u_{z}}{\partial z}(r, z), E_{r z}=\frac{1}{2}\left[\frac{\partial u_{r}}{\partial z}(r, z)+\frac{\partial u_{z}}{\partial r}(r, z)\right] .
\end{aligned}
$$

The relation (3.87) is, in detail,

$$
\left\{\begin{array}{l}
\Theta_{r r}=-\nu T+\lambda E+2 \mu E_{r r} \\
\Theta_{\phi \phi}=-\nu T+\lambda E+2 \mu E_{\phi \phi} \\
\Theta_{z z}=-\nu T+\lambda E+2 \mu E_{z z} \\
\Theta_{r z}=2 \mu E_{r z}
\end{array}\right.
$$

where, as above, $T(r, z)$ is the excess temperature field given by the generic $\mathrm{FB}$ expansion

$$
T(r, z)=\sum_{s} T_{s}(z) J_{0}\left(\zeta_{s} r / a\right)
$$

The stress tensor must obey the homogeneous divergence equation in the absence of external applied forces (static equilibrium), i.e., a special case of the Navier-Cauchy equations,

$$
\left\{\begin{array}{l}
\partial_{r} \Theta_{r r}+\left(\Theta_{r r}-\Theta_{\phi \phi}\right) / r+\partial_{z} \Theta_{r z}=0 \\
\left(\partial_{r}+1 / r\right) \Theta_{r z}+\partial_{z} \Theta_{z z}=0
\end{array} .\right.
$$

Moreover, the following boundary conditions must be satisfied:

$$
\Theta_{z z}(r, \pm h / 2)=0, \quad \Theta_{r z}(a, z)=0, \quad \Theta_{r z}(r, \pm h / 2)=0, \quad \Theta_{r r}(a, z)=0 .
$$

It is more convenient, at the end of the calculations, to express the results in terms of the Poisson ratio $\sigma$ and Young's modulus $Y$, using the correspondence

$$
\lambda=\frac{Y \sigma}{(1+\sigma)(1-2 \sigma)}, \quad \mu=\frac{Y}{2(1+\sigma)}, \quad \frac{\nu}{2(\lambda+\mu)}=\alpha(1+\sigma),
$$

where $\alpha$ is the linear thermal expansion coefficient.

\subsubsection{Thermal expansion from thermalization on the coating}

In the case of a heat source localized on the reflecting face, so that the temperature field $T(r, z)$ is known, it is possible to satisfy Equation (3.91) and all but one of the boundary conditions in Equation (3.92) by a displacement vector of the form

$$
u_{r}(r, z)=\frac{\nu}{2(\lambda+\mu)} \frac{1}{r} \int_{0}^{r} T\left(r^{\prime}, z\right) r^{\prime} d r^{\prime}
$$




$$
u_{z}(r, z)=\frac{\nu}{2(\lambda+\mu)}\left[\int_{-h / 2}^{z} T\left(r, z^{\prime}\right) d z^{\prime}+\Phi(r)\right],
$$

where $\Phi(r)$ is a function to be determined. It can be checked that $\Theta_{z z}=0$. The equilibrium equations then reduce to

$$
\left\{\begin{array}{l}
\partial_{z} \Theta_{r z}=0 \\
\left(\partial_{r}+1 / r\right) \Theta_{r z}=0
\end{array} .\right.
$$

We have

$$
\frac{\partial \Theta_{r z}}{\partial z}(r, z)=\frac{\nu \mu}{2(\lambda+\mu)}\left[\frac{\partial T}{\partial r}(r, z)+\frac{1}{r} \int_{0}^{r} \frac{\partial^{2} T}{\partial z^{2}}\left(r^{\prime}, z\right) r^{\prime} d r^{\prime}\right] .
$$

But let us recall that $T$ is harmonic [see Equation (3.1)], so that

$$
\frac{\partial^{2} T}{\partial z^{2}}=-\frac{1}{r} \frac{\partial}{\partial r}\left(r \frac{\partial T}{\partial r}\right)
$$

and consequently the first equilibrium equation (3.96) is identically satisfied

$$
\frac{\partial \Theta_{r z}}{\partial z}(r, z)=0
$$

Now we have

$$
\left(\partial_{r}+1 / r\right) \Theta_{r z}=\frac{\nu \mu}{2(\lambda+\mu)}\left[\Phi^{\prime \prime}(r)+\frac{1}{r} \Phi^{\prime}(r)+\frac{\partial T}{\partial z}(r,-h / 2)\right] .
$$

Therefore, in order to satisfy the second equilibrium equation (3.96), we take

$$
\Phi(r)=-\int_{0}^{r} \frac{d r^{\prime}}{r^{\prime}} \int_{0}^{r^{\prime}} \frac{\partial T}{\partial z}\left(r^{\prime \prime},-h / 2\right) r^{\prime \prime} d r^{\prime \prime}+C,
$$

where $C$ is an arbitrary constant. The stress component $\Theta_{r z}$ is now explicitly known

$$
\Theta_{r z}(r, z)=\frac{\nu \mu}{2(\lambda+\mu)}\left[\int_{-h / 2}^{z} \frac{\partial T}{\partial r}\left(r, z^{\prime}\right) d z^{\prime}+\frac{1}{r} \int_{0}^{r}\left(\frac{\partial T}{\partial z}\left(r^{\prime}, z\right)-\frac{\partial T}{\partial z}\left(r^{\prime},-h / 2\right)\right) r^{\prime} d r^{\prime}\right]
$$

making clear that $\Theta_{r z}(r,-h / 2)=0$, and since it has been shown that $\partial_{z} \Theta_{r z}(r, z)=0$, we have, simply,

$$
\Theta_{r z}=0 .
$$

Two more boundary conditions are satisfied. At this point, the only remaining unsatisfied condition is the vanishing of $\Theta_{r r}$ on the edge. Indeed, we have

$$
\Theta_{r r}(r, z)=-\frac{\nu \mu}{\lambda+\mu} \frac{1}{r^{2}} \int_{0}^{r} T\left(r^{\prime}, z\right) r^{\prime} d r^{\prime}
$$

or explicitly

$$
\Theta_{r r}(r, z)=-\frac{\nu \mu}{\lambda+\mu} \sum_{s>0} T_{s}(z) \frac{a}{\zeta_{s} r} J_{1}\left(\frac{\zeta_{s} r}{a}\right)
$$

so that

$$
\Theta_{r r}(a, z)=-\frac{\nu \mu}{\lambda+\mu} \sum_{s>0} T_{s}(z) \frac{J_{1}\left(\zeta_{s}\right)}{\zeta_{s}}
$$


It is easy to check numerically that the function $\Theta_{r r}(a, z)$ is almost linear for any type of beam. Therefore, it can be almost cancelled by an opposite linear stress on the edge. Such a stress can be induced by the following extra displacement vector:

$$
\begin{gathered}
\delta u_{r}(r, z)=\frac{\lambda+2 \mu}{2 \mu(3 \lambda+2 \mu)}\left(\omega_{0} r+\omega_{1} r z\right) \\
\delta u_{z}(r, z)=-\frac{\lambda}{\mu(3 \lambda+2 \mu)}\left(\omega_{0} z+\omega_{1} z^{2} / 2\right)-\frac{\lambda+2 \mu}{4 \mu(3 \lambda+2 \mu)} \omega_{1} r^{2},
\end{gathered}
$$

where $\omega_{0}$ and $\omega_{1}$ are arbitrary constants. One can check that this vector firstly satisfies the equilibrium equations (3.91), secondly has identically null stress components $\Theta_{r z}$ and $\Theta_{z z}$, and finally produces a radial edge stress:

$$
\delta \Theta_{r r}(a, z)=\omega_{0}+\omega_{1} z .
$$

The constants $\omega_{0}$ and $\omega_{1}$ can now be chosen in order to minimize the quadratic error:

$$
Q\left(\omega_{0}, \omega_{1}\right)=\int_{-h / 2}^{h / 2}\left[\Theta_{r r}(a, z)+\omega_{0}+\omega_{1} z\right]^{2} d z .
$$

After using the classical mean-squares formulas, this cancels the mean force and the mean torque on the edge:

$$
\begin{gathered}
\omega_{0}=-\frac{1}{h} \int_{-h / 2}^{h / 2} \Theta_{r r}(a, z) d z \\
\omega_{1}=-\frac{12}{h^{3}} \int_{-h / 2}^{h / 2} \Theta_{r r}(a, z) z d z .
\end{gathered}
$$

The complete displacement $\vec{U} \equiv \vec{u}+\delta \vec{u}$ now satisfies the Navier-Cauchy equations, all constraints on the faces, and induces null mean force and torque on the edge. Owing to the principle of de Saint-Venant [38], we can conclude that the displacement is correct almost everywhere in the bulk material, except possibly near the edge. But any effective optical beam has a vanishing intensity near the edge in order to prevent diffraction losses, so that the solution $\vec{U}$ is relevant for our purpose. If we use Young's modulus $Y$, the Poisson ratio $\sigma$ and the linear thermal expansion coefficient $\alpha$ instead of $\lambda, \mu, \nu$, we find

$$
\omega_{0}=\frac{\alpha Y \chi \epsilon P}{\pi K h} \sum_{s} p_{s} \frac{J_{0}\left(\zeta_{s}\right)}{\zeta_{s}^{3}} \frac{\sinh \gamma_{s}}{d_{1, s}},
$$

with $\gamma_{s} \equiv \zeta_{s} h / 2 a$. The FB coefficients $p_{s}$ are identical to the $p_{0, s}$ computed in Section 3.1.3 and

$$
\omega_{1}=-\frac{12 \alpha Y \chi \epsilon a P}{\pi K h^{3}} \sum_{s>0} p_{s} \frac{J_{0}\left(\zeta_{s}\right)}{\zeta_{s}^{4}} \frac{\gamma_{s} \cosh \gamma_{s}-\sinh \gamma_{s}}{d_{2, s}} .
$$

With the same notation, we have, explicitly, the components of the displacement:

$$
\begin{gathered}
u_{r}(r, z)=\alpha(1+\sigma) a \sum_{s>0} \frac{T_{s}(z)}{\zeta_{s}} J_{1}\left(\zeta_{s} r / a\right) \\
\delta u_{r}(r, z)=\frac{1-\sigma}{Y}\left(\omega_{0}+\omega_{1} z\right) r
\end{gathered}
$$




$$
\begin{gathered}
u_{z}(r, z)=\frac{\alpha(1+\sigma) \epsilon P}{2 \pi K} \sum_{s} \frac{p_{s}}{\zeta_{s}}\left[\frac{1}{d_{2, s}}+\left(\frac{\sinh \left(\zeta_{s} z / a\right)}{d_{1, s}}-\frac{\cosh \left(\zeta_{s} z / a\right)}{d_{2, s}}\right) J_{0}(\zeta r / a)\right] \\
\delta u_{z}(r, z)=-\frac{2 \sigma}{Y}\left[\omega_{0}+\frac{1}{2} \omega_{1} z\right] z-\frac{(1-\sigma)}{2 Y} \omega_{1} r^{2} .
\end{gathered}
$$

The displacement vector is defined up to a constant vector. We have chosen the constant in such a way that the displacement is zero at $(r=0, z=0)$. We can see in Figure 23 the global deformation of the mirror in the cases Ex1, Ex2 and Ex3.
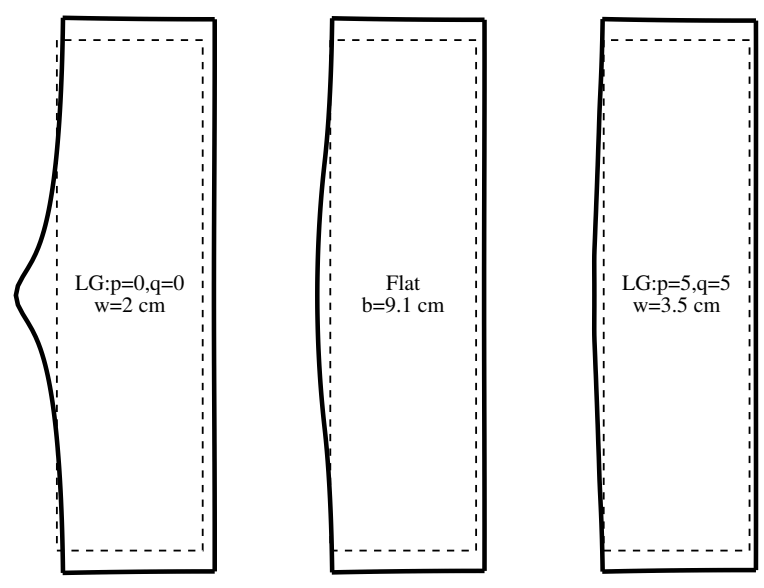

Figure 23: Thermal deformation of the mirror under three types of readout beams $(1 \mathrm{~W}$ absorbed power in the coating and exaggerated by a factor of $2 \times 10^{5}$ )

For the deformation of the coating, we have

$$
u_{z}(r,-h / 2)=\alpha(1+\sigma) \Phi(r)
$$

or, in detail,

$$
u_{z}(r,-h / 2)=\sum_{s>0} U_{s}\left[1-J_{0}\left(\zeta_{s} r / a\right)\right]
$$

with

$$
U_{s}=\frac{\alpha(1+\sigma) \epsilon P}{2 \pi K} \frac{p_{s}}{\zeta_{s}}\left(\frac{\sinh \gamma_{s}}{d_{1, s}}+\frac{\cosh \gamma_{s}}{d_{2, s}}\right) .
$$

(The displacement has now been taken to be zero at the center of the reflecting face), and

$$
\delta u_{z}(r,-h / 2)=-\frac{1-\sigma}{2 Y} \omega_{1} r^{2}
$$

The geometrical effects of heating (see Figure 23) are mainly a thermally-induced aberration due to the change of the reflecting surface by $u_{z}(r,-h / 2)+\delta u_{z}(r)$, then a change of the optical path through the substrate by a quantity

$$
\delta Z(r)=\left(n_{R}-1\right) \int_{-h / 2}^{h / 2} E_{z z}(r, z) d z=\alpha(1+\sigma)\left(n_{R}-1\right) \int_{-h / 2}^{h / 2} T(r, z) d z
$$


( $n_{R}$ being the nominal refractive index), which can be directly included in the thermal lens expression [Equation (3.40)], which has the same dependence on temperature. Note that the Saint-Venant correction contributes a constant (independent on $r$ ), so that we can ignore it in a lensing study. Thus, the global thermal lens is identical to the result found in Section 3, up to the correction

$$
\frac{d n}{d T} \rightarrow \frac{d n}{d T}+\alpha(1+\sigma)\left(n_{R}-1\right)
$$

Estimations of the weighted curvature of the distorted surface are obtained with the same technique as in Section 3. For Laguerre-Gauss modes, we obtain

$$
c=-\sum_{s>0} c_{s}^{(L G)} U_{s}^{(L G)}-\frac{1-\sigma}{2 Y} \omega_{1}^{(L G)} .
$$

For a flat mode, this is

$$
c=-\sum_{s>0} c_{s}^{(F)} U_{s}^{(F)}-\frac{1-\sigma}{2 Y} \omega_{1}^{(F)},
$$

where the coefficients $c_{s}$ have been defined by Equations (3.70) and (3.75).

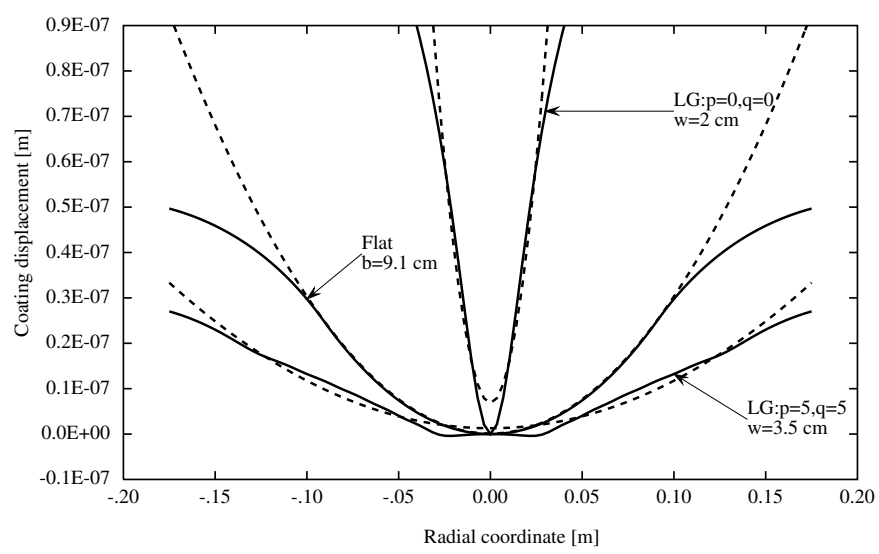

Figure 24: Deformation of the reflecting coating for three types of readout beams (coating absorption). Dashed line: best parabolic fit $\hat{u}_{z}+\delta \hat{u}_{z}=c r^{2}+d$ (weighted by the intensity profile) giving the effective curvature radius

We can see in Figure 24 the distorted reflecting face of the mirror in our three examples. The results in terms of curvature radius are

- $\operatorname{Ex1}\left(\mathrm{LG}_{0,0}, w=2 \mathrm{~cm}\right): R_{c}=5,842 \mathrm{~mW}$

- $\operatorname{Ex2}($ Flat, $b=9.1 \mathrm{~cm}): R_{c}=165,485 \mathrm{~mW}$

- $\operatorname{Ex} 3\left(\mathrm{LG}_{5,5}, w=3.5 \mathrm{~cm}\right): R_{c}=477,565 \mathrm{~mW}$.

We see in the case of axisymmetry that the use of unconventional modes (either flat or highorder LG) allows one to dramatically reduce spurious thermal effects in mirrors to be installed in advanced GW detectors, where high light-power flows are planned. Up to two orders of magnitude can be gained with respect to the present Virgo configuration for thermal lensing, or for thermal deformation of the coating. As in the thermal lens Section 3, we give some results (see Table 4) for LG modes having the same (1 ppm) clipping losses. 
Table 4: Curvature radii from thermal expansion due to coating absorption for modes having $1 \mathrm{ppm}$ clipping losses

\begin{tabular}{ccc}
\hline order $(p, q)$ & $\mathrm{w}[\mathrm{cm}]$ & $R_{c}$ of th. aberr. $[\mathrm{km} \mathrm{W}]$ \\
\hline$(0,0)$ & 6.65 & 77 \\
\hline$(0,1)$ & 5.56 & 149 \\
$(1,0)$ & 6.06 & 141 \\
\hline$(0,2)$ & 4.93 & 210 \\
$(1,1)$ & 5.23 & 201 \\
$(2,0)$ & 5.65 & 197 \\
\hline$(0,3)$ & 4.49 & 258 \\
$(1,2)$ & 4.70 & 250 \\
$(2,1)$ & 4.97 & 250 \\
$(3,0)$ & 5.35 & 250 \\
\hline$(0,4)$ & 4.17 & 299 \\
$(1,3)$ & 4.32 & 290 \\
$(2,2)$ & 4.52 & 292 \\
$(3,1)$ & 4.76 & 298 \\
$(4,0)$ & 5.11 & 300 \\
\hline$(0,5)$ & 3.91 & 332 \\
$(1,4)$ & 4.03 & 324 \\
$(2,3)$ & 4.18 & 326 \\
$(3,2)$ & 4.36 & 332 \\
$(4,1)$ & 4.58 & 342 \\
$(5,0)$ & 4.91 & 348 \\
\hline
\end{tabular}




\subsubsection{Thermal expansion from internal absorption}

When the linear absorption of light through the bulk material results in an internal heat source, the temperature field is no longer harmonic, and we are bound to solve explicitly the thermo-elastic equations (3.91) and (3.92). As seen earlier, the case of internal absorption leads to a symmetric temperature field. However, we shall derive the general thermoelastic solution, which will also prove useful in Section 4 below.

The temperature field is assumed to be of the generic form $\left(k_{s} \equiv \zeta / a\right)$

$$
T(r, z)=\sum_{s} T_{s}(z) J_{0}\left(k_{s} r\right) .
$$

We consider a displacement vector of the form

$$
\left\{\begin{array}{l}
u_{r}(r, z)=\sum_{s} A_{s}(z) J_{1}\left(k_{s} r\right) \\
u_{z}(r, z)=\sum_{s} B_{s}(z) J_{0}\left(k_{s} r\right) .
\end{array}\right.
$$

The equilibrium equations (3.91) reduce to a system of ordinary differential equations,

$$
\begin{gathered}
(\lambda+2 \mu)\left[\partial_{z}^{2} A_{s}-k_{s}^{2} A_{s}\right]-(\lambda+\mu) \partial_{z}\left(\partial_{z} A_{s}+k_{s} B_{s}\right)=-k_{s} \nu T_{s}(z) \\
(\lambda+2 \mu)\left[\partial_{z}^{2} B_{s}-k_{s}^{2} B_{s}\right]+(\lambda+\mu) k_{s}\left(\partial_{z} A_{s}+k_{s} B_{s}\right)=\nu \partial_{z} T_{s}(z),
\end{gathered}
$$

so that, by a basic combination of these two, we get

$$
\left[\partial_{z}^{2}-k_{s}^{2}\right]\left(\partial_{z} A_{s}+k_{s} B_{s}\right)=0
$$

a solution of which is

$$
\partial_{z} A_{s}+k_{s} B_{s}=k_{s} C_{s} \cosh \left(k_{s} z\right)+k_{s} D_{s} \sinh \left(k_{s} z\right)
$$

where $C_{s}$ and $D_{s}$ are arbitrary constants. By substituting in Equation (3.129) we get

$$
\left[\partial_{z}^{2}-k_{s}^{2}\right] A_{s}=\frac{\lambda+\mu}{\lambda+2 \mu} k_{s}^{2}\left(C_{s} \sinh \left(k_{s} z\right)+D_{s} \cosh \left(k_{s} z\right)\right) \cdot-k_{s} \frac{\nu}{\lambda+2 \mu} T_{s}(z)
$$

A solution of which is

$$
\begin{aligned}
A_{s}(z)= & M_{s} \sinh \left(k_{s} z\right)+N_{s} \cosh \left(k_{s} z\right)+\frac{\lambda+\mu}{2(\lambda+2 \mu)}\left[C_{s} k_{s} z \cosh \left(k_{s} z\right)+D_{s} k_{s} z \sinh \left(k_{s} z\right)\right] \\
& -\frac{\nu \mathcal{T}_{s}}{k_{s}(\lambda+2 \mu)},
\end{aligned}
$$

where $M_{s}$ and $N_{s}$ are two arbitrary constants and $\mathcal{T}_{s}(z)$ is a special solution of

$$
\left[\partial_{z}^{2}-k_{s}^{2}\right] \mathcal{T}_{s}(z)=T_{s}(z)
$$

We can now find $B_{s}$ from Equation (3.132)

$$
\begin{aligned}
B_{s}(z)= & {\left[C_{s}-M_{s}\right] \cosh \left(k_{s} z\right)+\left[D_{s}(z)-N_{s}(s)\right] \sinh \left(k_{s} z\right) } \\
& +\frac{\lambda+\mu}{2(\lambda+2 \mu)}\left[C_{s}\left(\cosh \left(k_{s} z\right)+k_{s} z \sinh \left(k_{s} z\right)\right)\right. \\
& \left.+D_{s}\left(\sinh \left(k_{s} z\right)+k_{s} z \cosh \left(k_{s} z\right)\right)\right]+\frac{\nu}{\lambda+2 \mu} \frac{\partial \mathcal{T}_{s}}{\partial z}(z) .
\end{aligned}
$$


The boundary conditions,

$$
\Theta_{r z}( \pm h / 2)=\Theta_{z z}( \pm h / 2)=0,
$$

lead to the system (we return to the Poisson ratio and to the linear thermal expansion coefficient)

$$
\begin{aligned}
4 \alpha(1+\sigma) \frac{\partial \mathcal{T}_{s}}{\partial z}(h / 2)= & {\left[\gamma_{s} \sinh \gamma_{s}-(1-2 \sigma) \cosh \gamma_{s}\right] C_{s}+\left[\gamma_{s} \cosh \gamma_{s}-(1-2 \sigma) \sinh \gamma_{s}\right] D_{s} } \\
& +4(1-\sigma) M_{s} \cosh \gamma_{s}+4(1-\sigma) N_{s} \sinh \gamma_{s} \\
4 \alpha(1+\sigma) \frac{\partial \mathcal{T}_{s}}{\partial z}(-h / 2)= & {\left[\gamma_{s} \sinh \gamma_{s}-(1-2 \sigma) \cosh \gamma_{s}\right] C_{s}-\left[\gamma_{s} \cosh \gamma_{s}-(1-2 \sigma) \sinh \gamma_{s}\right] D_{s} } \\
& +4(1-\sigma) M_{s} \cosh \gamma_{s}-4(1-\sigma) N_{s} \sinh \gamma_{s} \\
-4 \alpha(1+\sigma) k_{s} \mathcal{T}_{s}(h / 2)= & {\left[2(1-\sigma) \sinh \gamma_{s}-\gamma_{s} \cosh \gamma_{s}\right] C_{s}+\left[2(1-\sigma) \cosh \gamma_{s}-\gamma_{s} \sinh \gamma_{s}\right] D_{s} } \\
& -4(1-\sigma) M_{s} \sinh \gamma_{s}-4(1-\sigma) N_{s} \cosh \gamma_{s} \\
-4 \alpha(1+\sigma) k_{s} \mathcal{T}_{s}(-h / 2)= & -\left[2(1-\sigma) \sinh \gamma_{s}-\gamma_{s} \cosh \gamma_{s}\right] C_{s}+\left[2(1-\sigma) \cosh \gamma_{s}-\gamma_{s} \sinh \gamma_{s}\right] D_{s}+ \\
& 4(1-\sigma) M_{s} \sinh \gamma_{s}-4(1-\sigma) N_{s} \cosh \gamma_{s}
\end{aligned}
$$

with $\gamma_{s} \equiv k_{s} h / 2 \equiv \zeta_{s} h / 2 a$. It is easy to combine these equations to find

$$
\begin{gathered}
C_{s}=\frac{4 \alpha(1+\sigma)}{\Gamma_{s}^{\prime \prime}}\left(e^{\prime} s \sinh \gamma_{s}-k_{s} o_{s} \cosh \gamma_{s}\right) \\
D_{s}=\frac{4 \alpha(1+\sigma)}{\Gamma_{s}^{\prime}}\left(o^{\prime} s \cosh \gamma_{s}-k_{s} e_{s} \sinh \gamma_{s}\right) \\
M_{s}=\frac{\alpha(1+\sigma)}{(1-\sigma) \Gamma_{s}^{\prime \prime}}\left[\left(2(1-\sigma) \sinh \gamma_{s}-\gamma_{s} \cosh \gamma_{s}\right) e_{s}^{\prime}+\left(\gamma_{s} \sinh \gamma_{s}-(1-2 \sigma) \cosh \gamma_{s}\right) k_{s} o_{s}\right] \\
N_{s}=\frac{\alpha(1+\sigma)}{(1-\sigma) \Gamma_{s}^{\prime}}\left[\left(2(1-\sigma) \cosh \gamma_{s}-\gamma_{s} \sinh \gamma_{s}\right) o_{s}^{\prime}+\left(\gamma_{s} \cosh \gamma_{s}-(1-2 \sigma) \sinh \gamma_{s}\right) k_{s} e_{s}\right]
\end{gathered}
$$

with the notation

$$
\begin{gathered}
\Gamma_{s}^{\prime} \equiv \sinh \gamma_{s} \cosh \gamma_{s}+\gamma_{s}, \\
\Gamma_{s}^{\prime \prime} \equiv \sinh \gamma_{s} \cosh \gamma_{s}-\gamma_{s} .
\end{gathered}
$$

We have also used the symmetrized coefficients (even and odd parts)

$$
\left\{\begin{array}{l}
e_{s}=\frac{1}{2}\left(\mathcal{T}_{s}(h / 2)+\mathcal{T}_{s}(-h / 2)\right) \\
o_{s}=\frac{1}{2}\left(\mathcal{T}_{s}(h / 2)-\mathcal{T}_{s}(-h / 2)\right) \\
e^{\prime} s=\frac{1}{2}\left(\partial_{z} \mathcal{T}_{s}(h / 2)+\partial_{z} \mathcal{T}_{s}(-h / 2)\right) \\
o^{\prime} s=\frac{1}{2}\left(\partial_{z} \mathcal{T}_{s}(h / 2)-\partial_{z} \mathcal{T}_{s}(-h / 2)\right)
\end{array}\right.
$$

The radial stress is

$$
\Theta_{r r}(r, z)=\sum_{s} \Theta_{r r, s}^{(1)}(z) J_{0}\left(k_{s} r\right)-\sum_{s} \Theta_{r r, s}^{(2)}(z) \frac{J_{1}\left(k_{s} r\right)}{k_{s} r}
$$


with

$$
\begin{gathered}
\Theta_{r r, s}^{(1)}(z)=-\nu T_{s}(z)+(\lambda+2 \mu) k_{s} A_{s}(z)+\lambda \frac{\partial B_{s}}{\partial z}(z) \\
\Theta_{r r, s}^{(2)}(z)=2 \mu k_{s} A_{s}(z) .
\end{gathered}
$$

It can be checked that $\Theta_{r r, s}^{(1)}(z)$ gives a null contribution to the mean force and to the mean torque on the edge, i.e.,

$$
\int_{-h / 2}^{h / 2} \Theta_{r r, s}^{(1)}(z) d z=\int_{-h / 2}^{h / 2} \Theta_{r r, s}^{(1)}(z) z d z=0 .
$$

Therefore these two mean moments can be computed with $A_{s}(z)$. We have

$$
\begin{gathered}
\frac{1}{h} \int_{-h / 2}^{h / 2} A_{s}(z) d z=\alpha \frac{1+\sigma}{1-\sigma} \frac{2 \sigma}{\Gamma_{s}^{\prime}} \frac{\sinh \gamma_{s}}{\gamma_{s}}\left[k_{s} e_{s} \sinh \gamma_{s}-o_{s}^{\prime} \cosh \gamma_{s}\right] \\
+\alpha \frac{1+\sigma}{1-\sigma} \frac{1}{k_{s} h} \int_{-h / 2}^{h / 2} \mathcal{T}_{s}(z) d z \\
\frac{1}{h} \int_{-h / 2}^{h / 2} A_{s}(z) z d z=\alpha \frac{1+\sigma}{1-\sigma} \frac{2 \sigma}{\Gamma_{s}^{\prime \prime}}\left(\frac{\sinh \gamma_{s}}{\gamma_{s}}-\cosh \gamma_{s}\right)\left[e_{s}^{\prime} \sinh \gamma_{s}-k_{s} o_{s} \cosh \gamma_{s}\right] \\
+\alpha \frac{1+\sigma}{1-\sigma} \frac{1}{k_{s} h} \int_{-h / 2}^{h / 2} \mathcal{T}_{s}(z) z d z .
\end{gathered}
$$

In the special case of bulk absorption, we have, due to symmetry,

$$
o_{s}=e_{s}^{\prime}=0
$$

and

$$
\begin{gathered}
e_{s}=-\frac{\beta a^{2} P p_{s}}{\pi K \zeta^{4}}\left[1+\frac{\chi \gamma_{s} \sinh \gamma_{s}}{2 d_{1, s}}\right] \\
o_{s}^{\prime}=-\frac{\beta a P p_{s}}{\pi K \zeta^{3}}\left(\sinh \gamma_{s}+\gamma_{s} \cosh \gamma_{s}\right) .
\end{gathered}
$$

The explicit expression for functions $A_{s}(z)$ and $B_{s}(z)$ is, finally,

$$
\begin{aligned}
A_{s}(z)= & \alpha a \frac{1+\sigma}{1-\sigma} \frac{\alpha a \beta P}{\pi K \zeta_{s}^{3}}\left[1+\frac{\sinh \gamma_{s}}{\Gamma_{s}} k_{s} z \sinh \left(k_{s} z\right)\right. \\
& \left.-\left(\frac{\gamma_{s} \cosh \gamma_{s}-(1-2 \sigma) \sinh \gamma_{s}}{\Gamma_{s}}+(1-\sigma) \frac{\chi}{d_{1, s}}\right) \cosh \left(k_{s} z\right)\right] \\
B_{s}(z)= & \alpha a \frac{1+\sigma}{1-\sigma} \frac{\alpha a \beta P}{\pi K \zeta_{s}^{3}}\left[-\frac{\sinh \gamma_{s}}{\Gamma_{s}} k_{s} z \cosh \left(k_{s} z\right)\right. \\
& \left.+\left(\frac{\gamma_{s} \cosh \gamma_{s}+2(1-\sigma) \sinh \gamma_{s}}{\Gamma_{s}}-(1-\sigma) \frac{\chi}{d_{1, s}}\right) \sinh \left(k_{s} z\right)\right]
\end{aligned}
$$


Two boundary conditions have been forgotten. We need vanishing $\Theta_{r r}$ and $\Theta_{r z}$ on the edge $r=a$. However, a numerical investigation shows that $\Theta_{r r}(a, z)$ is practically constant, having an average value of

$$
\theta \equiv\left\langle\Theta_{r r}(a, z)\right\rangle=-\frac{\alpha a P Y \chi}{(1-\sigma) \pi K} \sum_{s} \frac{p_{s}}{\zeta_{s}^{4}}\left[1-\frac{\sinh \gamma_{s}}{\gamma_{s}}\left((1-\sigma) \frac{\chi}{d_{1, s}}+\frac{2 \sigma \sinh \gamma_{s}}{\Gamma_{s}}\right)\right] J_{0}\left(\zeta_{s}\right)
$$

In the same spirit as in the preceding case (Saint-Venant correction) we can add an extra displacement

$$
\begin{gathered}
\delta u_{r}(r, z)=-\frac{1-\sigma}{Y} \theta r \\
\delta u_{z}(r, z)=\frac{2 \sigma}{Y} \theta z
\end{gathered}
$$

which induces null $\delta \Theta_{r z}$ and $\delta \Theta_{z z}$ extra stresses, trivially satisfies the equilibrium equations, and produces a constant $\delta \Theta_{r r}=-\theta$. Now the $\Theta_{r z}$ stress component is antisymmetric with respect to $z$

$$
\Theta_{r z}(r, z)=\frac{\alpha Y}{1-\sigma} \sum_{s} \frac{T_{s}}{\Gamma_{s}}\left[k_{s} z \cosh \left(k_{s} z\right) \sinh \gamma_{s}-\gamma_{s} \cosh \gamma_{s} \sinh \left(k_{s} z\right)\right] J_{1}\left(k_{s} r\right)
$$

so that it is zero at $z= \pm h / 2$ with a vanishing average value on the edge $z \in[-h / 2, h / 2]$. Moreover, it can be checked that the values taken on the edge are weak compared to other places and other components. Therefore, the sum

$$
\vec{u}+\overrightarrow{\delta u}
$$

satisfies exactly the equilibrium equations, exactly the boundary conditions on the faces, and on average on the edge. The displacement vector at $z=-h / 2$ represents the deformation of the reflecting face. We have

$$
\begin{aligned}
Z(r) & =u_{z}(r,-h / 2)+\delta u_{z}(r,-h / 2) \\
& =\frac{\alpha(1+\sigma) \beta P a}{\pi K} \sum_{s} \frac{p_{s}}{\zeta_{s}^{3}} \sinh \gamma_{s}\left[\frac{2 \sinh \gamma_{s}}{\Gamma_{s}^{\prime}}-\frac{\chi}{d_{1, s}}\right] J_{0}\left(\zeta_{s} r / a\right)+\frac{2 \sigma}{Y} \theta z
\end{aligned}
$$

One can see in Figure 25 the distorted shape of the mirror in three situations. The thermallyinduced curvature radius can be computed as usual. (See Figure 26 for the profiles of the reflecting surface in three situations and the best fitted paraboloid.) For our three examples, we obtain the following figures

- $\operatorname{Ex1}\left(\mathrm{LG}_{0,0}, w=2 \mathrm{~cm}\right): R_{c}=22 \mathrm{~km} \mathrm{~W}$

- Ex2 (flat, $b=9.1 \mathrm{~cm}): R_{c}=325 \mathrm{~km} \mathrm{~W}($ mesa: $361 \mathrm{~km} \mathrm{~W}$ )

- $\operatorname{Ex} 3\left(\mathrm{LG}_{5,5}, w=3.5 \mathrm{~cm}\right): R_{c}=937 \mathrm{~km} \mathrm{~W}$

See Table 5 for several other LG modes.

In Table 6 we give numerical results for our three examples. 
Table 5: Curvature radii from thermal expansion due to bulk absorption for modes having 1 ppm clipping losses

\begin{tabular}{ccc}
\hline order $(p, q)$ & $w[\mathrm{~cm}]$ & $R_{c}$ of th. aberr. $[\mathrm{km} \mathrm{W}]$ \\
\hline$(0,0)$ & 6.65 & 165 \\
\hline$(0,1)$ & 5.56 & 318 \\
$(1,0)$ & 6.06 & 290 \\
\hline$(0,2)$ & 4.93 & 440 \\
$(1,1)$ & 5.23 & 410 \\
$(2,0)$ & 5.65 & 404 \\
\hline$(0,3)$ & 4.49 & 533 \\
$(1,2)$ & 4.70 & 507 \\
$(2,1)$ & 4.97 & 504 \\
$(3,0)$ & 5.35 & 512 \\
\hline$(0,4)$ & 4.17 & 613 \\
$(1,3)$ & 4.32 & 586 \\
$(2,2)$ & 4.52 & 585 \\
$(3,1)$ & 4.76 & 597 \\
$(4,0)$ & 5.11 & 615 \\
\hline$(0,5)$ & 3.91 & 677 \\
$(1,4)$ & 4.03 & 654 \\
$(2,3)$ & 4.18 & 650 \\
$(3,2)$ & 4.36 & 661 \\
$(4,1)$ & 4.58 & 684 \\
$(5,0)$ & 4.91 & 713 \\
\hline
\end{tabular}

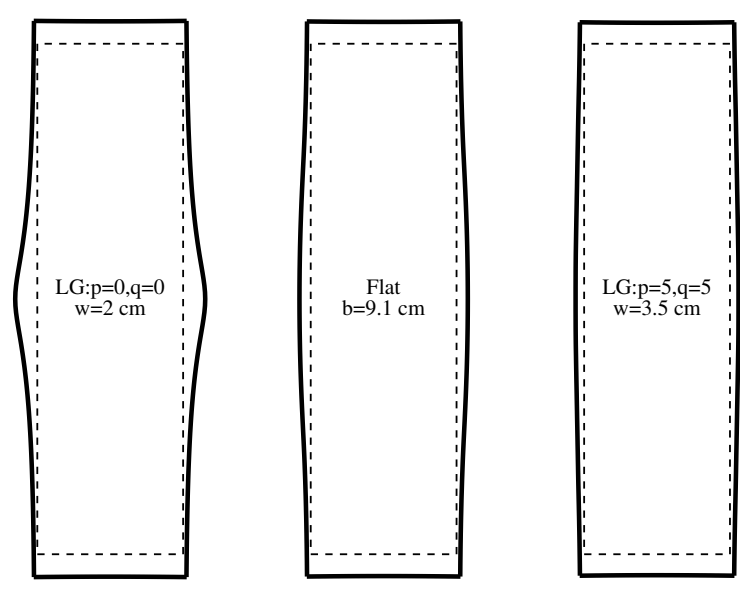

Figure 25: Thermal expansion of the mirror under three types of readout beams (heating by $1 \mathrm{~W}$ internal absorption of light, exaggerated by a factor of $2 \times 10^{5}$ ) 


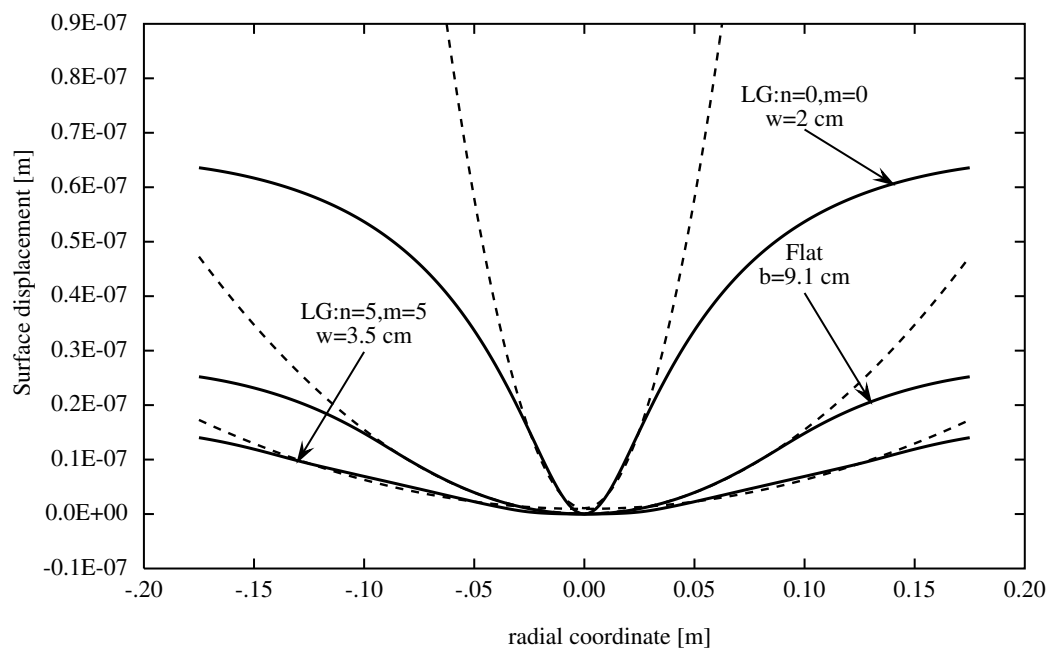

Figure 26: Thermal aberration caused by internal heating for $1 \mathrm{~W}$ absorbed power. Dashed lines: nearest paraboloid (weighted by the intensity distribution)

Table 6: Thermal aberrations from coating and bulk absorption

\begin{tabular}{cccc}
\hline results (coating abs.) & $\mathrm{LG}_{0,0} w=2 \mathrm{~cm}$ & flat $b=9.1 \mathrm{~cm}$ & $\mathrm{LG}_{5,5} w=3.5 \mathrm{~cm}$ \\
\hline Curvature radius & $5.8 \mathrm{~km} \mathrm{~W}$ & $167 \mathrm{~km} \mathrm{~W}$ & $478 \mathrm{~km} \mathrm{~W}$ \\
Coupling losses & $4.3 \times 10^{-2} / \mathrm{W}^{2}$ & $4.4 \times 10^{-3} / \mathrm{W}^{2}$ & $6.7 \times 10^{-3} / \mathrm{W}^{2}$ \\
\hline \hline results (bulk abs.) & $\mathrm{LG}_{0,0} w=2 \mathrm{~cm}$ & flat $b=9.1 \mathrm{~cm}$ & $\mathrm{LG}_{5,5} w=3.5 \mathrm{~cm}$ \\
\hline Curvature radius & $22 \mathrm{~km} \mathrm{~W}$ & $327 \mathrm{~km} \mathrm{~W}$ & $937 \mathrm{~km} \mathrm{~W}$ \\
Coupling losses & $3.0 \times 10^{-3} / \mathrm{W}^{2}$ & $2.2 \times 10^{-3} / \mathrm{W}^{2}$ & $1.8 \times 10^{-3} / \mathrm{W}^{2}$ \\
\hline
\end{tabular}




\subsection{Expansion on Zernike polynomials}

It is convenient to express the thermal lensing and the mirror distortions in terms of Zernike polynomials. This synthesizes the algebraic results already obtained and allows one to use the results, for instance, in optical simulation codes like the E2E by Caltech, Finesse at Birmingham (U.K.) and DarkF in Virgo. It is easy to find the coefficients of these polynomials. Recall that the axisymmetric Zernike polynomials $R_{2 n}^{0}(\rho)$ are functions of $\rho=r / a$, and that the first ones are

$$
R_{0}^{0}(\rho)=1, R_{2}^{0}(\rho)=2 \rho^{2}-1, R_{4}^{0}(\rho)=6 \rho^{4}-6 \rho^{2}+1
$$

corresponding respectively to the piston, curvature, and spherical aberration. A recurrence relation allows one to compute any order

$$
R_{2 n}^{0}(\rho)=\frac{1}{n}\left[(2 n-1)\left(2 \rho^{2}-1\right) R_{2 n-2}^{0}-(n-1) R_{2 n-2}^{0}\right] .
$$

An important relation is [6]

$$
\int_{0}^{1} R_{2 n}^{0}(\rho) J_{0}(\zeta \rho)=(-)^{n} \frac{J_{2 n+1}(\zeta)}{\zeta},
$$

which allows one to immediately create the Zernike expansion of any thermal lens. Assume a thermal lens of the generic FB form

$$
Z(r)=\sum_{s} Z_{s} J_{0}\left(\zeta_{s} r / a\right)
$$

It can also be represented by a series of Zernike polynomials as

$$
Z(r)=\sum_{n} c_{n} R_{2 n}^{0}(r / a)
$$

where the coefficients $c_{n}, n=0,1,2 \ldots$ are given by

$$
c_{n}=(-)^{n} 2(2 n+1) \sum_{s} Z_{s} \frac{J_{2 n+1}\left(\zeta_{s}\right)}{\zeta_{s}} .
$$

In the case of $\operatorname{Ex} 1\left(\mathrm{LG}_{00}, w=2 \mathrm{~cm}\right)$, we give, in order to be specific, some values of $c_{n}$ (see Table 7). The number of terms for a good reconstruction of the lenses and of the surface is about 15. The $c_{1}$ coefficient gives the mean curvature over the whole circular aperture of the mirror. Thus there is no relation between $c_{1}$ and the curvature averaged and weighted by the intensity profile.

Reconstruction of an $\mathrm{LG}_{00}$ mode requires many polynomials due to the sharp and far-fromspherical power profile of the beam. For higher-order modes and a fortiori for a flat or mesa mode, the reconstruction is achieved with fewer polynomials. In Table 8 we give the results of our $\mathrm{LG}_{55}$ mode (Ex3), and in Table 9 for the mesa mode (the figures are quite similar for the flat mode). 
Table 7: Zernike coefficients $c_{n}$ for $\mathrm{LG}_{00} w=2 \mathrm{~cm}$

\begin{tabular}{|c|c|c|c|c|}
\hline$n$ & $\begin{array}{l}\text { lensing: } \\
\text { heating } \\
\text { by bulk } \\
\mu \mathrm{m} / \mathrm{W}\end{array}$ & $\begin{array}{r}\text { lensing: } \\
\text { heating } \\
\text { by coating } \\
\mu \mathrm{m} / \mathrm{W}\end{array}$ & $\begin{array}{r}\text { aberration: } \\
\text { heating } \\
\text { by coating } \\
\mathrm{nm} / \mathrm{W}\end{array}$ & $\begin{array}{r}\text { aberration: } \\
\text { heating } \\
\text { by bulk } \\
\mathrm{nm} / \mathrm{W}\end{array}$ \\
\hline 0 & 0.940 & 0.873 & 140.76 & 25.85 \\
\hline 1 & -0.633 & -0.599 & 34.79 & -17.45 \\
\hline 2 & 0.470 & -0.447 & -25.60 & 12.25 \\
\hline 3 & -0.333 & -0.319 & 17.95 & -7.80 \\
\hline 4 & 0.250 & 0.239 & -13.42 & 5.06 \\
\hline 5 & -0.192 & -0.185 & 10.38 & -3.35 \\
\hline 6 & 0.151 & 0.146 & -8.17 & 2.27 \\
\hline 7 & -0.120 & -0.116 & 6.50 & -1.57 \\
\hline 8 & 0.095 & 0.092 & -5.19 & 1.10 \\
\hline 9 & -0.076 & -0.074 & 4.14 & -0.79 \\
\hline 10 & 0.060 & 0.059 & -3.30 & 0.57 \\
\hline 11 & -0.048 & -0.047 & 2.61 & -0.41 \\
\hline 12 & 0.038 & 0.037 & -2.06 & -0.30 \\
\hline 13 & -0.029 & -0.029 & 1.61 & -0.21 \\
\hline 14 & 0.023 & 0.022 & -1.25 & 0.16 \\
\hline
\end{tabular}

Table 8: Zernike coefficients $c_{n}$ for $L_{55} w=3.5 \mathrm{~cm}$

\begin{tabular}{r|rrrr}
\hline & $\begin{array}{r}\text { lensing: } \\
\text { heating } \\
\text { by bulk }\end{array}$ & $\begin{array}{r}\text { lensing: } \\
\text { heating } \\
\text { by coating } \\
\mu \mathrm{m} / \mathrm{W}\end{array}$ & $\begin{array}{r}\text { aberration: } \\
\text { heating } \\
\text { by coating } \\
\mathrm{nm} / \mathrm{W}\end{array}$ & $\begin{array}{r}\text { aberration: } \\
\text { heating } \\
\text { by bulk } \\
\mathrm{nm} / \mathrm{W}\end{array}$ \\
\hline 0 & 0.817 & 0.865 & 16.48 & 24.21 \\
1 & -0.229 & -0.242 & 13.00 & -6.64 \\
2 & 0.044 & 0.046 & -2.67 & 1.38 \\
3 & -0.019 & -0.019 & 1.058 & -0.42 \\
4 & 0.021 & 0.022 & -1.198 & 0 \\
5 & -0.002 & -0.003 & 0.135 & 0 \\
6 & -0.006 & -0.006 & 0.314 & 0 \\
7 & 0.001 & 0.001 & -0.047 & 0 \\
8 & -0.002 & -0.002 & 0.091 & 0 \\
9 & 0.006 & 0.007 & -0.356 & 0 \\
10 & -0.006 & -0.006 & 0.315 & 0 \\
\hline
\end{tabular}


Table 9: Zernike coefficients $c_{n}$ for the mesa mode

\begin{tabular}{r|rrrr}
\hline & $\begin{array}{l}\text { lensing: } \\
\text { heating } \\
\text { by bulk } \\
\mu \mathrm{m} / \mathrm{W}\end{array}$ & $\begin{array}{r}\text { lensing: } \\
\text { heating } \\
\text { by coating } \\
\mu \mathrm{m} / \mathrm{W}\end{array}$ & $\begin{array}{r}\text { aberration: } \\
\text { heating } \\
\text { by coating } \\
\mathrm{nm} / \mathrm{W}\end{array}$ & $\begin{array}{r}\text { aberration: } \\
\text { heating } \\
\text { by bulk } \\
\mathrm{nm} / \mathrm{W}\end{array}$ \\
\hline 0 & 0.845 & 0.895 & 30.81 & 25.05 \\
1 & -0.387 & -0.408 & 22.25 & -11.19 \\
2 & 0.147 & 0.155 & -8.55 & 4.12 \\
3 & -0.185 & -0.020 & 0 & 0 \\
4 & -0.011 & -0.012 & 0 & 0 \\
5 & 0.008 & 0.008 & 0 & 0 \\
\hline
\end{tabular}

\section{On Thermal Compensation Systems}

It is easily seen that in the case of a $\mathrm{TEM}_{00}$ readout beam, even with the largest possible $w$ allowed by clipping losses, the resulting thermal lens is currently a serious spurious effect and will be even greater in advanced interferometers. For a fundamental mode with a width of $6.65 \mathrm{~cm}$ on the cavity input mirrors, the curvature radius of the thermal lens is about $4 \mathrm{~km}$ for $1 \mathrm{~W}$ thermalized, on the same order as the nominal curvature radius of the mirror.

This is why thermal compensation systems (TCS) have been designed. The general principle is to use an auxiliary source of heat for heating the cold parts of the mirror in order to achieve a more homogeneous temperature distribution. The source of heat may be a classical radiator able to radiate infrared energy in a vacuum, for instance, a hot ring near the rear face of the mirror [30, 25, 13, 14, 22, 43]. It may also be an auxiliary laser projector, which can be programmed to scan the mirror surface to produce a given power mask [42].

\subsection{Heating the rear face of a mirror}

Technical issues make it difficult to install any device in front of the cavity input mirrors. Thus, it has generally been proposed that one heat the rear face of these mirrors, which is more accessible from the central part of the vacuum tank. The heating is due to infrared radiation produced either by a hot material or by a $\mathrm{CO}_{2}$ auxiliary laser, but in both cases, the wavelength of the heating radiator is such that absorption by the silica substrate occurs in a thin layer. If we assume another heat source located on the rear face of a mirror, we must modify the model developed above. The extension is straightforward. For instance, let us consider the case in which the thermal lens to be compensated for is caused by thermalization on the coating of the intracavity-stored power $P$. As usual, we denote by $p_{s}$ the $\mathrm{FB}$ coefficients related to the main readout beam and by $P$ and $p_{C, s}$ the FB coefficients of the power distribution of the compensation system, radiating an integrated power $P_{C}$. We get the following temperature field

$$
T(r, z)=\sum_{s} T_{s}(z) J_{0}\left(k_{s} r\right)
$$

with

$$
\begin{aligned}
T_{s}(z)= & \frac{1}{\pi a K} \frac{1}{\left(\zeta_{s}+\chi\right)^{2}-\left(\zeta_{s}+\chi\right)^{2} \mathrm{e}^{-4 \gamma_{s}}} \times\left\{\mathrm{e}^{-\gamma_{s}}\left[\left(\zeta_{s}-\chi\right) \mathrm{e}^{k_{s} z-2 \gamma_{s}}+\left(\zeta_{s}+\chi\right) \mathrm{e}^{-k_{s} z}\right] P p_{s}\right. \\
& \left.+\mathrm{e}^{-3 \gamma_{s}}\left[\left(\zeta_{s}+\chi\right) \mathrm{e}^{k_{s} z+2 \gamma_{s}}+\left(\zeta_{s}-\chi\right) \mathrm{e}^{-k_{s} z}\right] P_{C} p_{C, s}\right\} .
\end{aligned}
$$


(For brevity, we have set $k_{s} \equiv \zeta_{s} / a$ and $\gamma_{s} \equiv k_{s} h / 2$, with $\zeta_{s}$ defined as before). By integrating Equation (4.2) on the thickness, we get the thermal lens

$$
Z(r)=\frac{d n}{d T} \int_{-h / 2}^{h / 2} T(r, z) d z
$$

which yields

$$
Z(r)=\sum_{s} Z_{s} J_{0}\left(k_{s} r\right)
$$

with

$$
Z_{s}=\frac{d n}{d T} \frac{1}{\pi K} \frac{\sinh \gamma_{s}}{\zeta_{s}} \frac{P p_{s}+P_{C} p_{C, s}}{d_{1, s}}
$$

with $d_{1, s}=\zeta_{s} \sinh \gamma_{s}+\chi \cosh \gamma_{s}$. As expected, this shows that the global thermal lens is simply the sum of the primary lens (caused by the stored light) plus the compensation lens. Thus, it is possible to imagine power profiles compensating for the primary lens. This is the starting point for thermal compensation systems.

\subsection{Simple model of a radiator}

Now assume a compensation system based on a ring radiator of radius $b_{C}$, at a distance $D_{C}$ from the face, placed behind the rear face of the mirror. Assume a total radiated power of $P_{R}$. A small element of length $d l$ of the ring (assumed very thin) radiates the elementary power $d P=$ $P_{C} \times d l / 2 \pi b_{C}$. The radiated intensity at a distance $\rho$ is

$$
d I=\frac{d P}{4 \pi \rho^{2}}=\frac{P_{R} d l}{8 \pi^{2} b_{C} \rho^{2}} .
$$

Now the distance of the considered element, having the angular coordinate $\phi$ on the ring to any point $\mathrm{A}$ on the mirror surface of coordinates $A(r, \Phi)$, is such that

$$
\rho^{2}=b_{C}^{2}+D_{C}^{2}+r^{2}-2 b_{C} r \cos (\phi-\Phi),
$$

so that the global intensity at $\mathrm{A}$, integrated on the ring, is

$$
I(r)=\frac{P_{R}}{8 \pi^{2}} \int_{0}^{2 \pi} \frac{d \phi}{b_{C}^{2}+D_{C}^{2}+r^{2}+2 b_{C} r \cos \phi}
$$

which gives

$$
I(r)=\frac{P_{R}}{4 \pi} \frac{1}{\sqrt{\left(b_{C}^{2}+D_{C}^{2}\right)^{2}-2\left(b_{C}^{2}-D_{C}^{2}\right) r^{2}+r^{4}}} .
$$

The fraction of the total radiated power, which is absorbed by the rear face of the mirror, is

$$
\Delta P=2 \pi \int_{0}^{a} I(r) r d r=\frac{1}{4} P_{R} \ln \left\{\frac{b_{C}}{D_{C}}\left[\sqrt{\left(\frac{a^{2}+D_{C}^{2}-b_{C}^{2}}{2 b_{C} D_{C}}\right)^{2}+1}+\frac{a^{2}+D_{C}^{2}-b_{C}^{2}}{2 b_{C} D_{C}}\right]\right\}
$$

(provided that $b_{c}>D_{C}$ ) This allows one to normalize the intensity distribution to $P_{C}$ integrated on the mirror:

$$
I(r)=P_{C} \frac{I_{0}}{\sqrt{\left(b_{C}^{2}+D_{C}^{2}\right)^{2}-2\left(b_{C}^{2}-D_{C}^{2}\right) r^{2}+r^{4}}}
$$




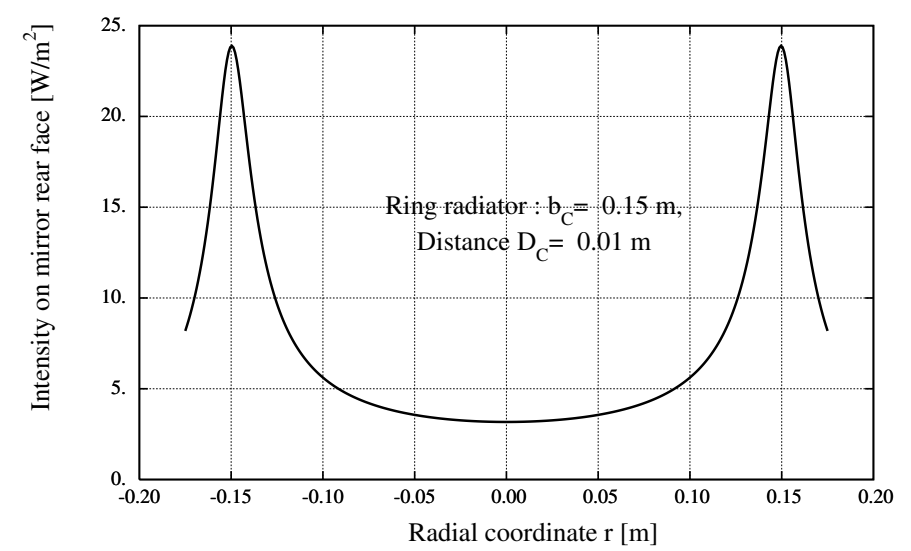

Figure 27: Normalized intensity $I(r)$ on the mirror rear face from a ring radiator

Table 10: Thermal compensation with a heating ring: $\mathrm{LG}_{0,0}$ mode of $w=2 \mathrm{~cm}$.

\begin{tabular}{rrccc}
\hline dissipated power & initial losses & compensation power & minimal losses & wavefront curvature \\
\hline $10 \mathrm{~mW}$ & $350 \mathrm{ppm}$ & $2.7 \mathrm{~W}$ & $24 \mathrm{ppm}$ & $\infty$ \\
$20 \mathrm{~mW}$ & $1,400 \mathrm{ppm}$ & $5.3 \mathrm{~W}$ & $96 \mathrm{ppm}$ & $\infty$ \\
$30 \mathrm{~mW}$ & $3,100 \mathrm{ppm}$ & $8.0 \mathrm{~W}$ & $220 \mathrm{ppm}$ & $\infty$ \\
$100 \mathrm{~mW}$ & $34,300 \mathrm{ppm}$ & $26.5 \mathrm{~W}$ & $2392 \mathrm{ppm}$ & $\infty$ \\
\hline
\end{tabular}

with

$$
\frac{1}{I_{0}}=\pi \ln \left\{\frac{b_{C}}{D_{C}}\left[\sqrt{\left(\frac{a^{2}+D_{C}^{2}-b_{C}^{2}}{2 b_{C} D_{C}}\right)^{2}+1}+\frac{a^{2}+D_{C}^{2}-b_{C}^{2}}{2 b_{C} D_{C}}\right]\right\}
$$

See Figure 27 to get an idea of the intensity profile.

The FB coefficients of $I(r)$ can easily be numerically computed. The resulting profile of the thermal lens is shown for a particular case in Figure 28.

The power in the compensation lens must be adjusted in order to get zero curvature when added to the heat source coming from the readout beam. For small readout-beam dissipation (less than a few $\mathrm{mW}$ ), this minimizes the matching losses. An example is given in Figure 29 in which one tries to compensate for the thermal lens caused by an $\mathrm{LG}_{00}$ mode of width $w=2 \mathrm{~cm}$ dissipating either 10, 20 or $30 \mathrm{~mW}$ on the coating. One sees that, by increasing the compensation power, it is possible to reduce the coupling losses from their initial (uncompensated) values by a factor of about 15 (see Table 10). However, it can be seen that the residual loss is proportional to the dissipated power, which means that, in the case of cavities storing about $1 \mathrm{MW}$, even with thermalization rates on the order of $1 \mathrm{ppm}$, the system is useless. For $100 \mathrm{~mW}$ dissipated, one can see that the TCS is able to reduce the initial losses of almost $3.5 \%$ to about $0.24 \%$, which is still much too high, and at the price of $26.5 \mathrm{~W}$ TCS power dissipated.

In the latter case, we see in Figure 30 that even if the mean curvature radius is infinite, it does not mean that the lens is exactly flat, so that, even if the focusing effect is suppressed, some losses are to be expected due to departure of the lens from a plane (or from a sphere having a large 


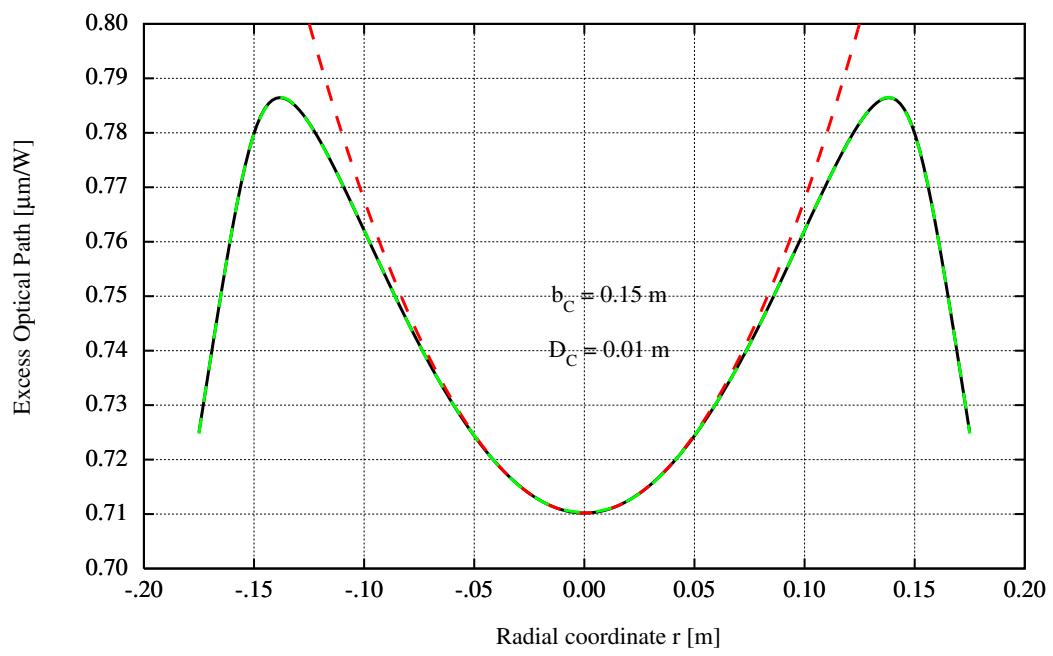

Figure 28: Thermal lensing from a ring radiator. Red dashed curve: nearest paraboloid (weighted by the readout beam intensity). The readout beam is $\mathrm{TEM}_{00}$ with $w=2 \mathrm{~cm}$. The curvature is weakly dependent on the beam width: $87 \mathrm{kmW}$ for $w=2 \mathrm{~cm}, 95 \mathrm{kmW}$ for $w=6.65 \mathrm{~cm}$. Green dashed curve: Zernike expansion of the lens

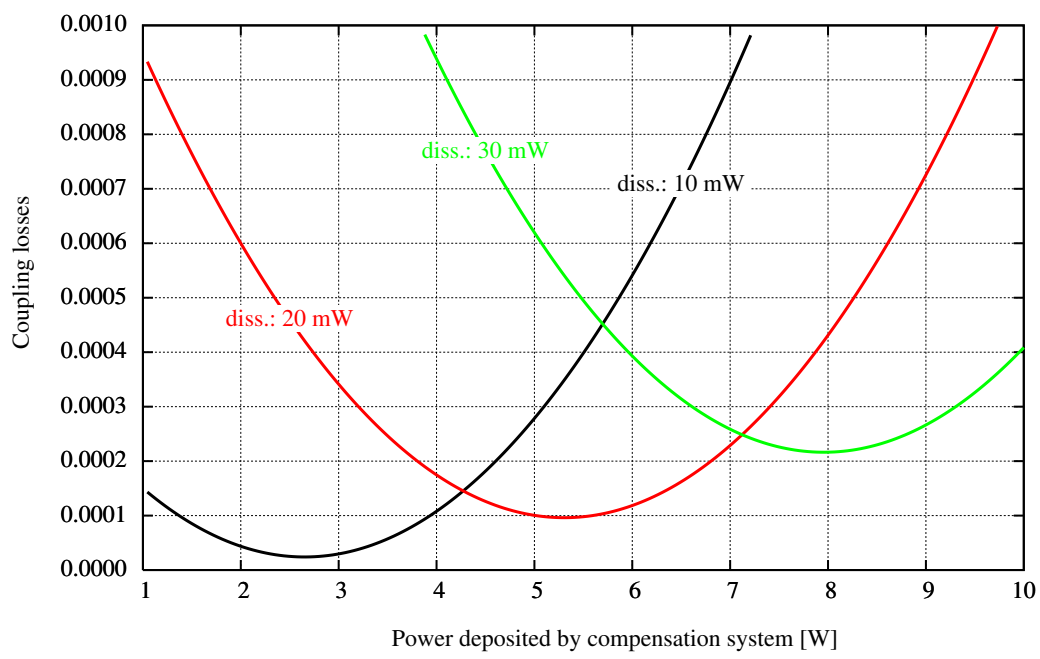

Figure 29: Thermal compensation with a ring radiator: minimization of coupling losses 
Table 11: Thermal compensation with a heating ring: $\mathrm{LG}_{5,5}$ mode of $w=3.5 \mathrm{~cm}$.

\begin{tabular}{rrrrr}
\hline dissipated power & initial losses & compensation power & minimal losses & wavefront curvature \\
\hline $10 \mathrm{~mW}$ & $56 \mathrm{ppm}$ & $50 \mathrm{~mW}$ & $6 \mathrm{ppm}$ & $\infty$ \\
$20 \mathrm{~mW}$ & $213 \mathrm{ppm}$ & $100 \mathrm{~mW}$ & $11 \mathrm{ppm}$ & $\infty$ \\
$30 \mathrm{~mW}$ & $474 \mathrm{ppm}$ & $130 \mathrm{~mW}$ & $15 \mathrm{ppm}$ & $\infty$ \\
$100 \mathrm{~mW}$ & $5,218 \mathrm{ppm}$ & $450 \mathrm{~mW}$ & $122 \mathrm{ppm}$ & $\infty$ \\
\hline
\end{tabular}

radius). Only six Zernike polynomials are required to retrieve this special TCS lens:

$$
\begin{gathered}
c_{0}=0.759 \mu \mathrm{m} / W, c_{1}=0.016 \mu \mathrm{m} / W, c_{2}=-0.044 \mu \mathrm{m} / W, \\
c_{3}=-0.011 \mu \mathrm{m} / W, c_{4}=0.002 \mu \mathrm{m} / W, c_{5}=0.004 \mu \mathrm{m} / W .
\end{gathered}
$$

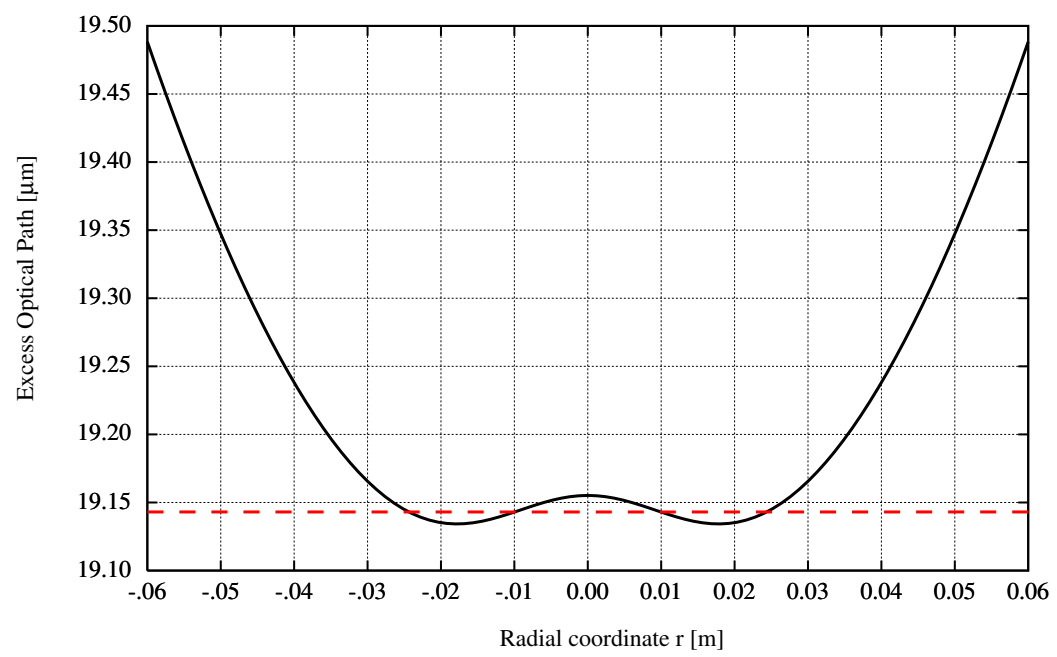

Figure 30: Ring radiator: correction of the thermal lensing caused by a TEM To $_{0}$ beam of width $w=2 \mathrm{~cm}$ dissipating $100 \mathrm{~mW}$ on the coating. Dashed line: nearest paraboloid (flat for the optimal TCS power of $26.5 \mathrm{~W})$

It is probably possible to enhance these results up to a certain extent by tuning the parameters $b_{C}$ and $D_{C}$, but not to seriously change the orders of magnitude. However, results are better with higher-order modes (see Table 11).

\subsection{Axicon systems}

Some systems use $\mathrm{CO}_{2}$ laser beams focused by a conical lens. This configuration, called "axicon", generates a ring-shaped intensity profile on the mirror. We do not attempt to compute an analytical model of the compensation power flow. We use numerical intensity measurements carried out by the Virgo/Rome team [15]. The FB coefficients of the optical power flow are computed numerically. The profile of the corresponding thermal lens can be seen in Figure 31.

We can see (Table 12) that the performance of the axicon system is better than that of the heating ring (lower compensating powers, lower optimal losses), but not by orders of magnitude. 


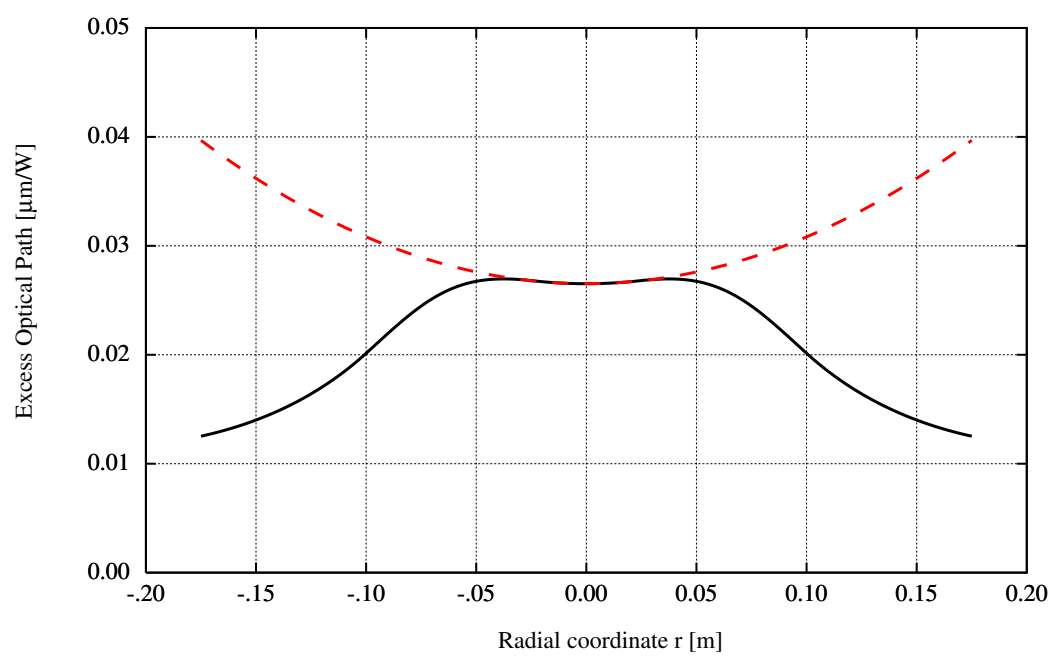

Figure 31: Thermal lens profile created by an axicon system

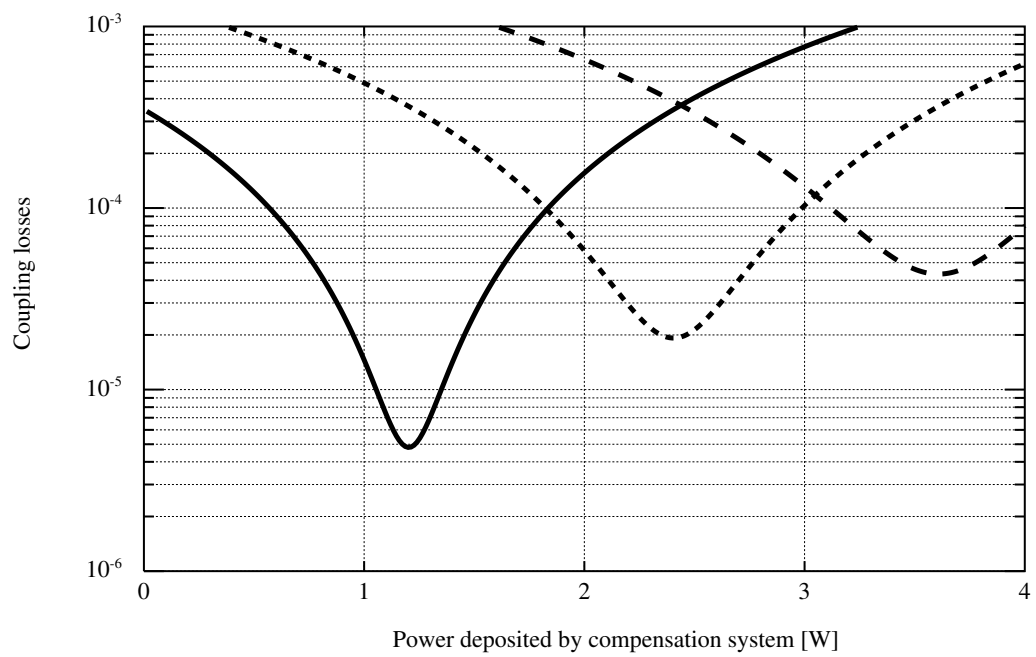

Figure 32: Thermal compensation with an axicon: minimization of coupling losses. Solid line, short dashed, long dashed: resp. $10 \mathrm{~mW}, 20 \mathrm{~mW}, 30 \mathrm{~mW}$, dissipated by the readout beam.

Table 12: Thermal compensation with an axicon system

\begin{tabular}{rrrrc}
\hline dissipated power & initial losses & compensation power & minimal losses & wavefront curvature \\
\hline $10 \mathrm{~mW}$ & $350 \mathrm{ppm}$ & $1.2 \mathrm{~W}$ & $5 \mathrm{ppm}$ & $\infty$ \\
$20 \mathrm{~mW}$ & $1,400 \mathrm{ppm}$ & $2.4 \mathrm{~W}$ & $19 \mathrm{ppm}$ & $\infty$ \\
$30 \mathrm{~mW}$ & $3,100 \mathrm{ppm}$ & $3.6 \mathrm{~W}$ & $43 \mathrm{ppm}$ & $\infty$ \\
$100 \mathrm{~mW}$ & $34,300 \mathrm{ppm}$ & $12 \mathrm{~W}$ & $481 \mathrm{ppm}$ & $\infty$ \\
\hline
\end{tabular}




\section{4 $\mathrm{CO}_{2}$ laser compensation by scanning}

By scanning the rear face with a powerful $\mathrm{CO}_{2}$ laser, it is possible, in principle, to obtain any given intensity profile. In particular, it is possible to obtain an intensity distribution giving a flat thermal lens over a large central part of the mirror. Consider an intensity profile of the form

$$
I(r)=P_{C} \frac{\cosh \left(r^{2} / w_{C}^{2}\right)}{\pi w_{C}^{2} \sinh \left(a^{2} / w_{C}^{2}\right)}
$$

depending on the only parameter $w_{C}$ and normalized to $P_{C} \mathrm{~W}$ (see Figure 33 for the case of $\left.w_{C}=16.9 \mathrm{~cm}\right)$.

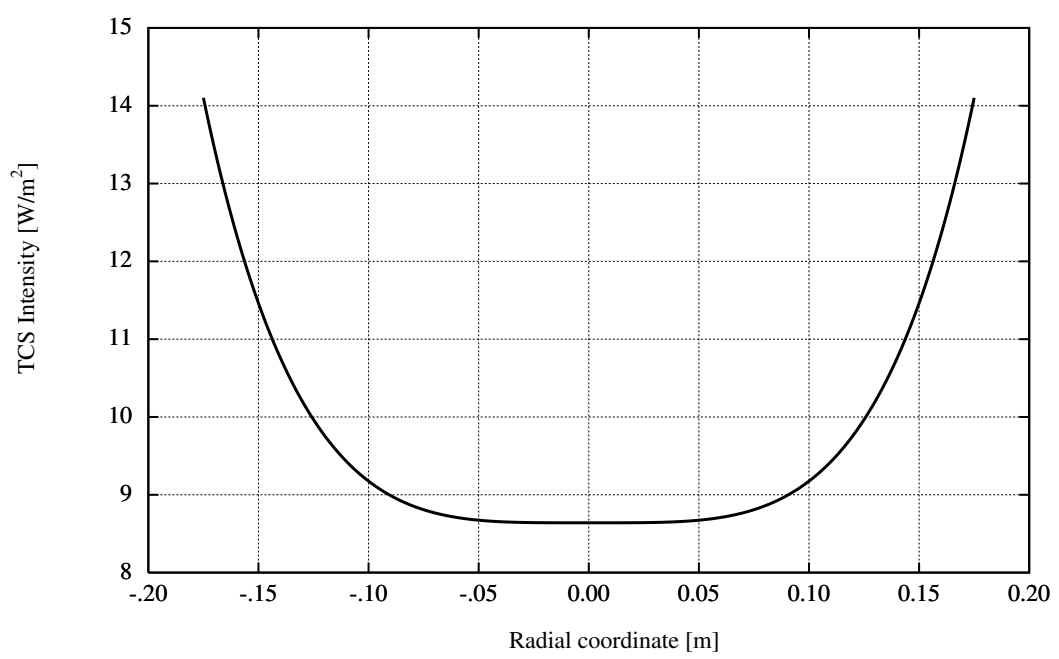

Figure 33: Source of heat on the mirror rear face for a power mask according to Equation (4.14) with $w_{C}=16.9 \mathrm{~cm}$ (normalized to $\left.1 \mathrm{~W}\right)$

The resulting thermal lens has a perfect flatness in the central region. The goal is to create a profile such that, combined with the readout beam heat source, it gives that ideal profile. Consider, for instance, an intensity mask of the form

$$
I_{C}(r)=\frac{2 P_{L}}{\pi w^{2}}\left[\cosh \left(r^{2} / w_{C}^{2}\right)-\exp \left(-r^{2} / w^{2}\right)\right]
$$

see the profile on Figure 34. This is nothing but the complement to a source of heat corresponding to a $\mathrm{TEM}_{00}$ mode dissipating $P_{L} \mathrm{~W}$ on the coating. The resulting global thermal lens (thus corrected) can be seen in Figure 35.

The price to pay is to provide the correcting power. According to Equation (4.15), the integrated power of the mask is

$$
P_{C}=2 \pi \int_{0}^{a} I_{C}(r) r d r=2 P_{L}\left[\frac{w_{C}^{2}}{w^{2}} \sinh \left(a^{2} / w_{C}^{2}\right)-1\right] .
$$

We see in this rather academic case (Table 13) that the residual losses are much less than in the preceding case (by a factor of about 20), but at the price of higher TCS power. The expansion in terms of Zernike polynomials is given in Table 14. 


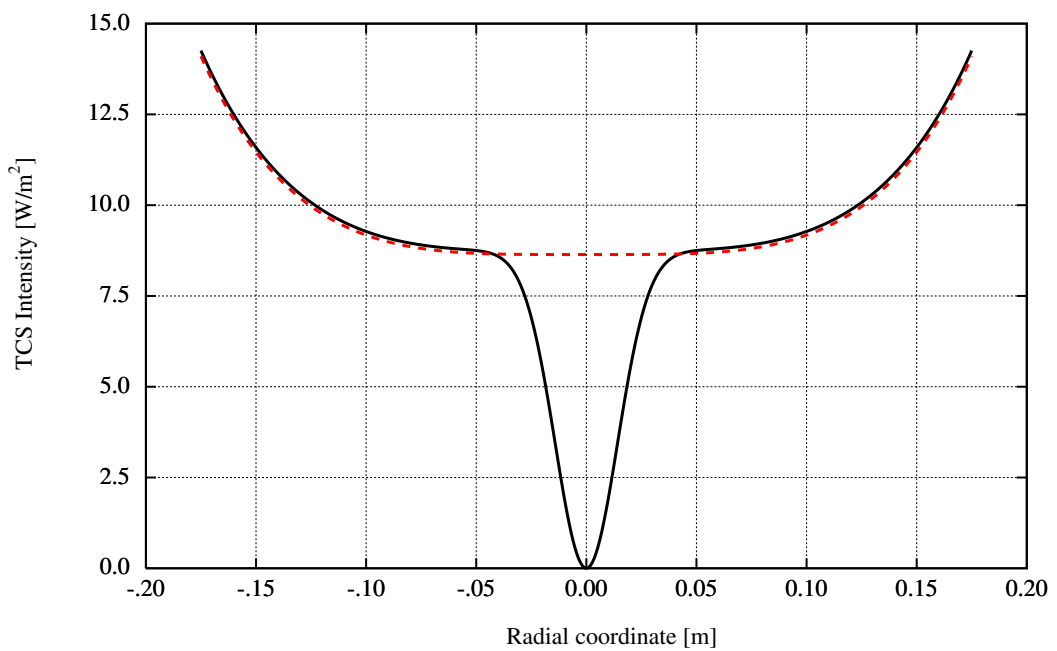

Figure 34: Source of heat on the mirror rear face for a power mask according to Equation (4.15) with $w_{C}=16.9 \mathrm{~cm}$ for $1 \mathrm{~W}$ dissipated by the readout beam

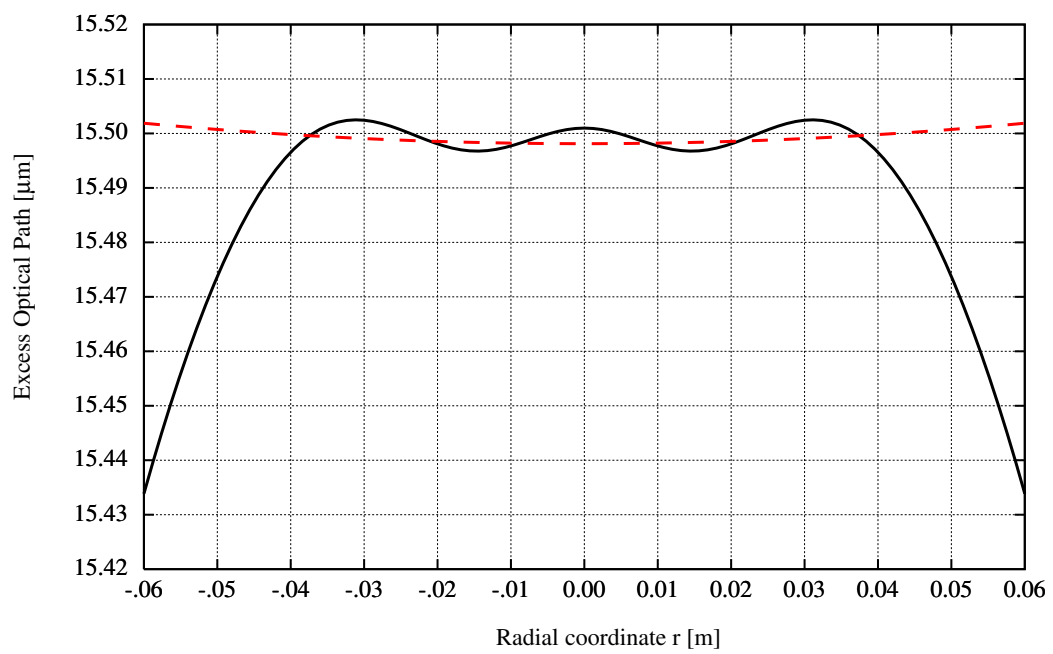

Figure 35: Corrected thermal lens by a power mask according to Equation (4.15) with $w_{C}=16.9 \mathrm{~cm}$ for $100 \mathrm{~mW}$ dissipated by the readout $\mathrm{LG}_{00}$ beam $(w=2 \mathrm{~cm})$

Table 13: Thermal compensation with a scanning $\mathrm{CO}_{2}$ beam for $\mathrm{LG}_{00}$ mode $(w=2 \mathrm{~cm})$

\begin{tabular}{rrrrr}
\hline dissipated power & initial losses & compensation power & minimal losses & wavefront curvature \\
\hline $10 \mathrm{~mW}$ & $350 \mathrm{ppm}$ & $1.9 \mathrm{~W}$ & $0.7 \mathrm{ppm}$ & $\infty$ \\
$20 \mathrm{~mW}$ & $1,400 \mathrm{ppm}$ & $3.8 \mathrm{~W}$ & $3 \mathrm{ppm}$ & $\infty$ \\
$30 \mathrm{~mW}$ & $3,100 \mathrm{ppm}$ & $5.6 \mathrm{~W}$ & $6.4 \mathrm{ppm}$ & $\infty$ \\
$100 \mathrm{~mW}$ & $34,300 \mathrm{ppm}$ & $18.8 \mathrm{~W}$ & $71 \mathrm{ppm}$ & $\infty$ \\
\hline
\end{tabular}


Table 14: Zernike coefficients for three TCS systems compensating $\mathrm{LG}_{00}$ mode $(w=2 \mathrm{~cm})$

\begin{tabular}{c|rrr}
\hline$c_{n}$ & $\begin{array}{r}\text { heating ring } \\
\mu \mathrm{m} / \mathrm{W}\end{array}$ & $\begin{array}{r}\text { Axicon } \\
\mu \mathrm{m} / \mathrm{W}\end{array}$ & $\begin{array}{r}\mathrm{CO}_{2} \text { scan } \\
\mu \mathrm{m} / \mathrm{W}\end{array}$ \\
\hline 0 & 0.759 & 0.018 & 0.774 \\
1 & 0.016 & -0.008 & -0.058 \\
2 & -0.044 & 0.003 & -0.016 \\
3 & -0.012 & 0.001 & 0.001 \\
4 & 0.002 & -0.001 & -0.002 \\
5 & 0.004 & 0.001 & 0.002 \\
6 & 0.002 & 0 & -0.001 \\
7 & 0 & 0 & 0.001 \\
8 & -0.001 & 0 & -0.001 \\
\hline
\end{tabular}

\section{Heating in the Quasistatic Regime: Heating from Cold}

It is interesting to study the temperature evolution of a mirror, assuming a constant light power flux switched on at $t=0$.

\subsection{Transient temperature field}

We consider the time-dependent heat equation with an internal source of heat (bulk absorption of light) and a surface source on the coating. The Fourier equation is

$$
\left[\rho C \partial_{t}-K \Delta\right] T(t, r, z)=S_{1}(r)
$$

where $\rho$ is the density of the material and $C$ its specific heat. $S_{1}$ accounts for the dissipation of light passing through the substrate. As in the static case, it is independent on $z$ because the attenuation of the beam is extremely weak; moreover, we assume it is independent of time. In a recycling interferometer, we would expect the transient lens to change the reflectance of the cavity, the recycling rate, in turn, and eventually the source $S_{1}$, so that $S_{1}$ is a function of $t$. But we consider here an isolated mirror. The case of a complex system with feedback can be treated sequentially (see [19]) using any propagation code, but this is beyond our present goal. For instance, we assume some servo loop acting to maintain a constant power flow. The boundary conditions are still

$$
-K\left[\frac{\partial T}{\partial z}\right]_{r, z=-h / 2}=-4 \sigma_{\mathrm{f}} T_{0}^{3} T(r,-h / 2)+S_{2}(r),
$$

where $S_{2}$ accounts for the dissipation of light on the reflective coating. The next two boundary conditions are

$$
-K\left[\frac{\partial T}{\partial z}\right]_{r, z=h / 2}=4 \sigma_{\mathrm{f}} T_{0}^{3} T(r, h / 2)
$$

and

$$
-K\left[\frac{\partial T}{\partial r}\right]_{r=a, z}=4 \sigma_{\text {edge }} T_{0}^{3} T(a, z) .
$$

We already know the steady state solution $T_{\infty}$ for coating or bulk dissipation in the general form

$$
T_{\infty}(r, z)=\sum_{s} T_{s}(z) J_{0}\left(k_{s} r\right)
$$


where $k_{s} \equiv \zeta_{s} / a$ and $\left\{\zeta_{s}: s=1,2, ..\right\}$ have the same definition as in Equation (3.11). $T_{\infty}(r, z)$ satisfies all boundary conditions plus the inhomogeneous heat equation. Thus, it is a special solution of Equation (5.1). We now look for a general time-dependent solution of the homogeneous heat equation

$$
\left[\rho C \partial_{t}-K \Delta\right] T_{\operatorname{tr}}(t, r, z)=0
$$

and satisfying homogeneous boundary conditions. We search this transient temperature field under the form

$$
T_{\mathrm{tr}}(t, r, z)=\sum_{s, j}\left[\theta_{s, j}^{\prime}(t) \cos \left(\kappa_{j}^{\prime} z\right)+\theta_{s, j}^{\prime \prime}(t) \sin \left(\kappa_{j}^{\prime \prime} z\right)\right] J_{0}\left(k_{n} r\right),
$$

so that the general solution of Equation (5.1) will be

$$
T(t, r, z)=T_{\infty}(r, z)+T_{\operatorname{tr}}(t, r, z) .
$$

Now, the time functions $\theta_{s, j}^{\prime}(t)$ and $\theta_{s, j}^{\prime \prime}(t)$ must satisfy

$$
\frac{\partial \theta_{s, j}^{\prime}}{\partial t}+\frac{K}{\rho C}\left(k_{n}^{2}+\kappa_{j}^{\prime 2}\right) \theta_{s, j}^{\prime}=0
$$

and

$$
\frac{\partial \theta_{s, j}^{\prime \prime}}{\partial t}+\frac{K}{\rho C}\left(k_{n}^{2}+\kappa_{j}^{\prime \prime 2}\right) \theta_{s, j}^{\prime \prime}=0
$$

whose solutions are

$$
\begin{aligned}
& \theta_{s, j}^{\prime}(t)=\theta_{s, j}^{\prime} \exp \left(-t / \tau_{s, j}^{\prime}\right) \\
& \theta_{s, j}^{\prime \prime}(t)=\theta_{s, j}^{\prime \prime} \exp \left(-t / \tau_{s, j}^{\prime \prime}\right),
\end{aligned}
$$

where the time constants are, respectively,

with the main time constant

$$
\begin{aligned}
\tau_{s, j}^{\prime} & =\frac{\tau}{\gamma_{s}^{2}+u_{k}^{2}} \\
\tau_{s, j}^{\prime \prime} & =\frac{\tau}{\gamma_{s}^{2}+v_{j}^{2}}
\end{aligned}
$$

$$
\tau \equiv \frac{\rho C h^{2}}{4 K}
$$

(about $0.8 \mathrm{~h}$ for a regular Virgo mirror substrate). The boundary conditions on the faces lead to the following equations:

$$
\begin{aligned}
\kappa_{j}^{\prime} \frac{h}{2} \sin \left(\kappa_{j}^{\prime} \frac{h}{2}\right)-\frac{4 \sigma_{f} T_{0}^{3} h}{2 K} \cos \left(\kappa_{j}^{\prime} \frac{h}{2}\right)=0 \\
\kappa_{j}^{\prime \prime} \frac{h}{2} \cos \left(\kappa_{j}^{\prime \prime} \frac{h}{2}\right)+\frac{4 \sigma_{f} T_{0}^{3} h}{2 K} \sin \left(\kappa_{j}^{\prime \prime} \frac{h}{2}\right)=0 .
\end{aligned}
$$

In terms of the reduced radiation constant $\chi^{\prime}=\chi h / 2 a$, the first equation is of the form

$$
u \sin u-\chi^{\prime} \cos u=0 .
$$

This equation admits an infinite discrete family of solutions $\left\{u_{j}, j \in \mathbb{N}^{*}\right\}$, giving us $\kappa_{l}^{\prime}=2 u_{j} / h$. In the same way, we have $\kappa_{j}^{\prime \prime}=2 v_{j} / h$, where the constants $\left\{v_{j}, j \in \mathbb{N}^{*}\right\}$ are all solutions of

$$
v \cos v+\chi^{\prime} \sin v=0 \text {. }
$$


Owing again to the Sturm-Liouville theorem, the functions $\left\{\cos \left(2 u_{j} z / h\right), j \in \mathbb{N}^{*}\right\}$ form a complete orthogonal set, and a basis for symmetric functions of $z$ defined in $[-h / 2, h / 2]$. Functions $\left\{\sin \left(2 v_{j} z / h\right), j \in \mathbb{N}^{*}\right\}$ form also a complete and orthogonal set for antisymmetric functions on $[-h / 2, h / 2]$. The two sets are obviously mutually orthogonal. Moreover, we have

$$
\int_{-h / 2}^{h / 2} \cos \left(\kappa_{j}^{\prime} z\right) \cos \left(\kappa_{j^{\prime}}^{\prime} z\right) d z=g_{j}^{\prime} \delta_{j j^{\prime}}
$$

with the normalization constant

$$
g_{j}^{\prime}=\frac{h}{2}\left[1+\frac{\sin \left(2 u_{j}\right)}{2 u_{j}}\right]
$$

and in the same way

$$
\int_{-h / 2}^{h / 2} \cos \left(\kappa_{j}^{\prime \prime} z\right) \cos \left(\kappa_{j^{\prime}}^{\prime \prime} z\right) d z=g_{j}^{\prime \prime} \delta_{j j^{\prime}}
$$

with

$$
g_{j}^{\prime \prime}=\frac{h}{2}\left[1-\frac{\sin \left(2 v_{j}\right)}{2 v_{j}}\right] .
$$

At this point, all constants have been determined, except $\theta_{s, j}^{\prime}$ and $\theta_{s, j}^{\prime \prime}$. This is done depending on the initial conditions on the temperature. The total temperature field being

$$
\begin{aligned}
T(t, r, z) & =T_{\infty}(r, z)+T_{\operatorname{tr}}(t, r, z) \\
& =\sum_{s}\left[T_{s}(z)+\sum_{j}\left(\theta_{s, j}^{\prime}(t) \cos \left(\kappa_{j}^{\prime} z\right)+\theta_{s, j}^{\prime \prime}(t) \sin \left(\kappa_{j}^{\prime \prime} z\right)\right)\right] J_{0}\left(k_{s} r\right),
\end{aligned}
$$

Thus, the initial temperature is

$$
\begin{aligned}
T(0, r, z) & =T_{\infty}(r, z)+T_{\mathrm{tr}}(t, r, z) \\
& =\sum_{s}\left[T_{s}(z)+\sum_{j}\left(\theta_{s, j}^{\prime} \cos \left(\kappa_{j}^{\prime} z\right)+\theta_{s, j}^{\prime \prime} \sin \left(\kappa_{j}^{\prime \prime} z\right)\right)\right] J_{0}\left(k_{s} r\right),
\end{aligned}
$$

and if we require heating from room temperature, i.e., $T(0, r, z)=0$, considering the orthogonality of the functions $J_{0}\left(k_{s} r\right)$, we are led to the equation

$$
T_{s}(z)+\sum_{j}\left[\theta_{s, j}^{\prime} \cos \left(\kappa_{j}^{\prime}\right)+\theta_{s, j}^{\prime \prime} \sin \left(\kappa_{j}^{\prime \prime}\right)\right]=0
$$

giving

$$
\begin{aligned}
& \theta_{s, j}^{\prime}=-\frac{1}{g_{j}^{\prime}} \int_{-h / 2}^{h / 2} T_{s}(z) \cos \left(\kappa_{j}^{\prime} z\right) d z \\
& \theta_{s, j}^{\prime \prime}=-\frac{1}{g_{j}^{\prime \prime}} \int_{-h / 2}^{h / 2} T_{s}(z) \sin \left(\kappa_{j}^{\prime \prime} z\right) d z,
\end{aligned}
$$

which completes the determination. Finally, the temperature field is

$$
T(t, r, z)=-\sum_{s, j}\left[\theta_{s, j}^{\prime}\left(1-\mathrm{e}^{-t / \tau_{s, j}^{\prime}}\right) \cos \left(\kappa_{j}^{\prime} z\right)+\theta_{s, j}^{\prime \prime}\left(1-\mathrm{e}^{-t / \tau_{s, j}^{\prime \prime}}\right) \sin \left(\kappa_{j}^{\prime \prime} z\right)\right] J_{0}\left(k_{s} r\right) .
$$

It is now possible to specialize the result to the two cases of coating and bulk absorption. 


\subsubsection{Transient temperature from coating absorption}

In the case of coating absorption, we know from Equation (3.18) specialized to axisymmetry that

$$
T_{s}(z)=\frac{\epsilon P}{2 \pi K a} p_{s}\left[\frac{\cosh \left(\zeta_{s} z / a\right)}{d_{1, s}}-\frac{\sinh \left(\zeta_{s} z / a\right)}{d_{2, s}}\right],
$$

$\left(\gamma_{s} \equiv \zeta_{s} h / 2 a\right)$. After some elementary algebra, we have

$$
\begin{aligned}
\theta_{s, j}^{\prime} & =-\frac{\epsilon P h p_{s}}{2 \pi K a^{2}} \frac{\cos u_{j}}{1+\sin \left(2 u_{j}\right) / 2 u_{j}} \frac{1}{u_{j}^{2}+\gamma_{s}^{2}} \\
\theta_{s, j}^{\prime \prime} & =\frac{\epsilon P h p_{s}}{2 \pi K a^{2}} \frac{\sin v_{j}}{1-\sin \left(2 v_{j}\right) / 2 v_{j}} \frac{1}{v_{j}^{2}+\gamma_{s}^{2}}
\end{aligned}
$$

so that the final result for the transient temperature field is

$$
\begin{aligned}
T(t, r, z) & =\frac{\epsilon P h}{2 \pi K a^{2}} \sum_{s, j} p_{s}\left[\frac{\cos \left(u_{j}\right)}{\left(1+\sin \left(2 u_{j}\right) / 2 u_{j}\right)\left(u_{j}^{2}+\gamma_{s}^{2}\right)}\left(1-\mathrm{e}^{-t / \tau_{s, j}^{\prime}}\right) \cos \left(\kappa_{j}^{\prime} z\right)\right. \\
& \left.-\frac{\sin \left(v_{j}\right)}{\left(1-\sin \left(2 v_{j}\right) / 2 v_{j}\right)\left(v_{j}^{2}+\gamma_{s}^{2}\right)}\left(1-\mathrm{e}^{-t / \tau_{s, j}^{\prime \prime}}\right) \sin \left(\kappa_{j}^{\prime \prime} z\right)\right] J_{0}\left(k_{s} r\right)
\end{aligned}
$$

The transient thermal lens is

$$
\begin{aligned}
Z(t, r) & =\frac{d n}{d T} \int_{-h / 2}^{h / 2} T(t, r, z) d z \\
& =\frac{d n}{d T} \frac{\epsilon P h^{2}}{2 \pi K a^{2}} \sum_{s, j} p_{s} \frac{\sin \left(2 u_{j}\right) / 2 u_{j}}{\left(1+\sin \left(2 u_{j}\right) / 2 u_{j}\right)\left(u_{j}^{2}+\gamma_{s}^{2}\right)}\left(1-\mathrm{e}^{-t / \tau_{s, j}^{\prime}}\right) J_{0}\left(k_{s} r\right) .
\end{aligned}
$$

Figures 36,37 , and 38 show the time evolution of the thermal lens. The time constant for temperature evolution is very long, several hours, but the time constant of the focal length of the thermal lens is much shorter because the final profile of the lens is reached within about $1 / 2 \mathrm{~h}$, after which the temperature keeps growing uniformly without changing the gradients.

\subsubsection{Transient temperature from bulk absorption}

In the case of internal absorption, we have

$$
T_{s}(z)=\frac{\beta P}{\pi K} \frac{p_{s}}{\zeta_{s}^{2}}\left[1-\frac{\chi \cosh \left(k_{s} z\right) \cdot p n g}{d_{1, s}}\right] .
$$

We have, obviously, $\theta_{s, j}^{\prime \prime}=0$ and, after some algebra, we find

$$
\theta_{s, j}^{\prime}=-\frac{\beta P p_{s} h^{2}}{2 \pi K a^{2}} \frac{\sin u_{j} / u_{j}}{\left(1+\sin \left(2 u_{j}\right) / 2 u_{j}\right)\left(\gamma_{s}^{2}+u_{j}^{2}\right)},
$$

so that the temperature field is

$$
T(t, r, z)=\frac{\beta P h^{2}}{2 \pi K a^{2}} \sum_{s, j} p_{s} \frac{\sin u_{j} / u_{j}}{\left(1+\sin \left(2 u_{j}\right) / 2 u_{j}\right)\left(\gamma_{s}^{2}+u_{j}^{2}\right)}\left(1-\mathrm{e}^{-t / \tau_{s, j}^{\prime}}\right) \cos \left(\kappa_{j}^{\prime} z\right) J_{0}\left(k_{s} r\right)
$$




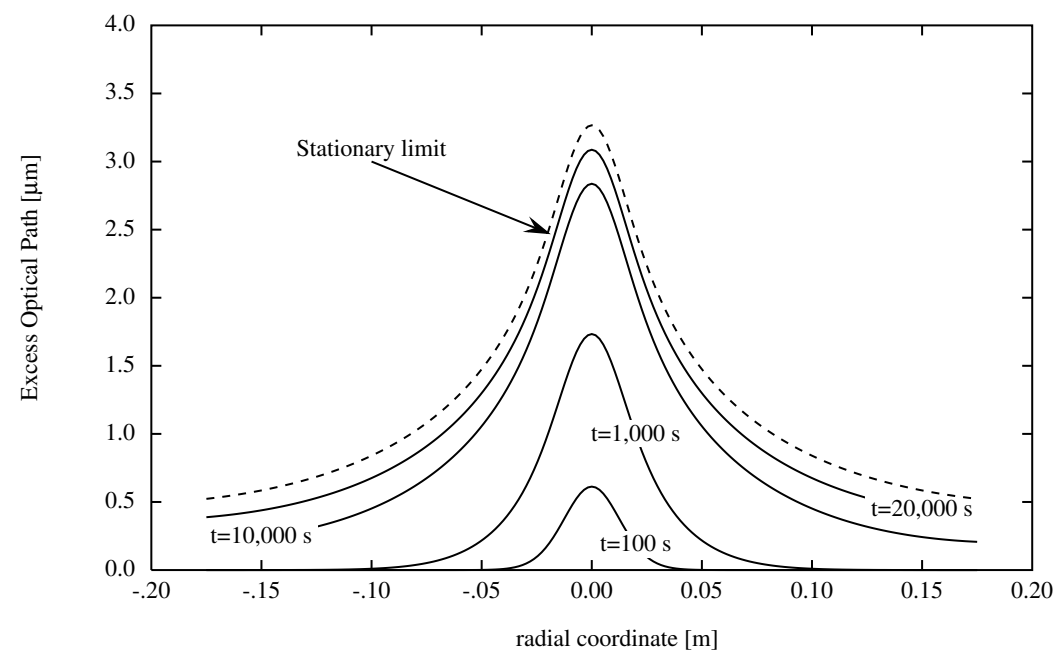

Figure 36: Time evolution of the thermal lens from room temperature to the steady state limit. Heating from coating absorption, $\mathrm{LG}_{0,0}$ mode, $w=2 \mathrm{~cm}$

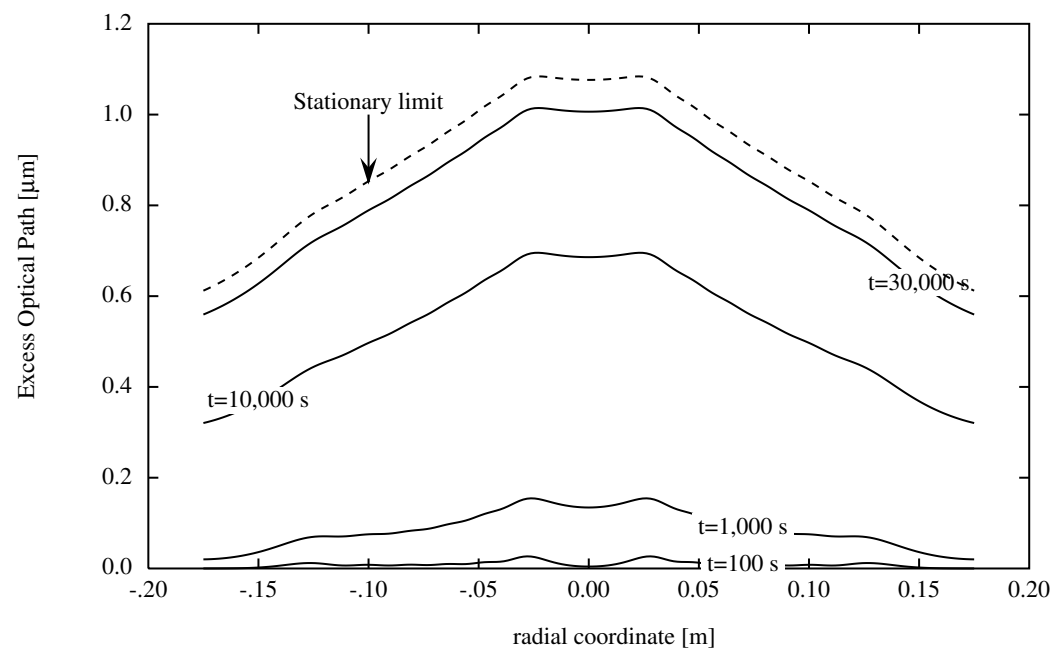

Figure 37: Time evolution of the thermal lens from room temperature to steady state limit. Heating from coating absorption, $\mathrm{LG}_{5,5}$ mode, $w=3.5 \mathrm{~cm}$ 


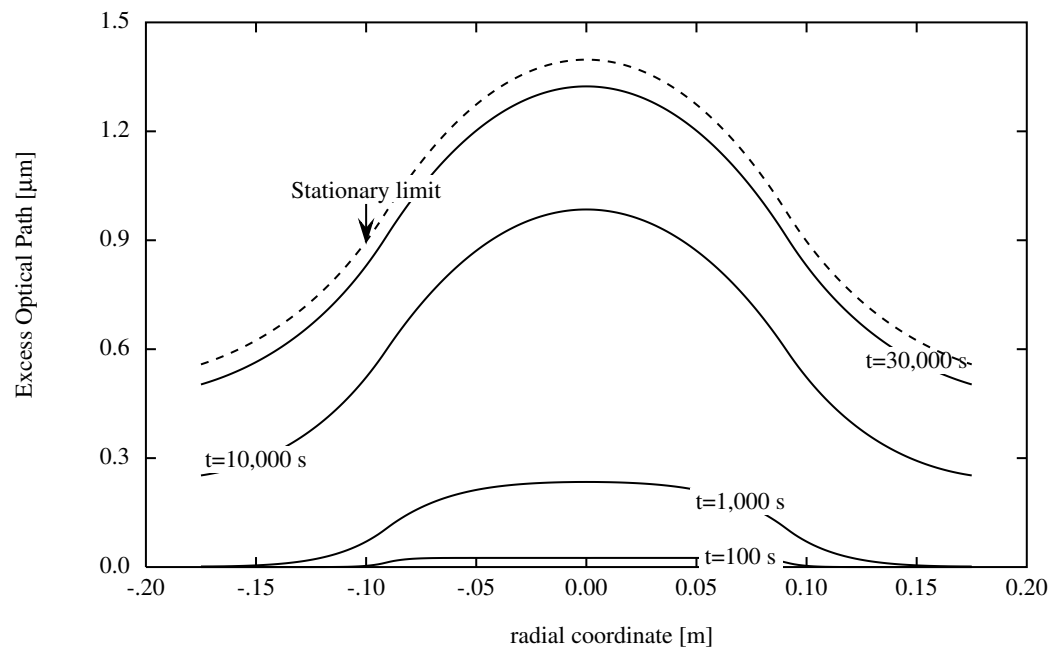

Figure 38: Time evolution of the thermal lens from room temperature to the steady state limit. Heating from coating absorption, Flat mode, $b=9.1 \mathrm{~cm}$

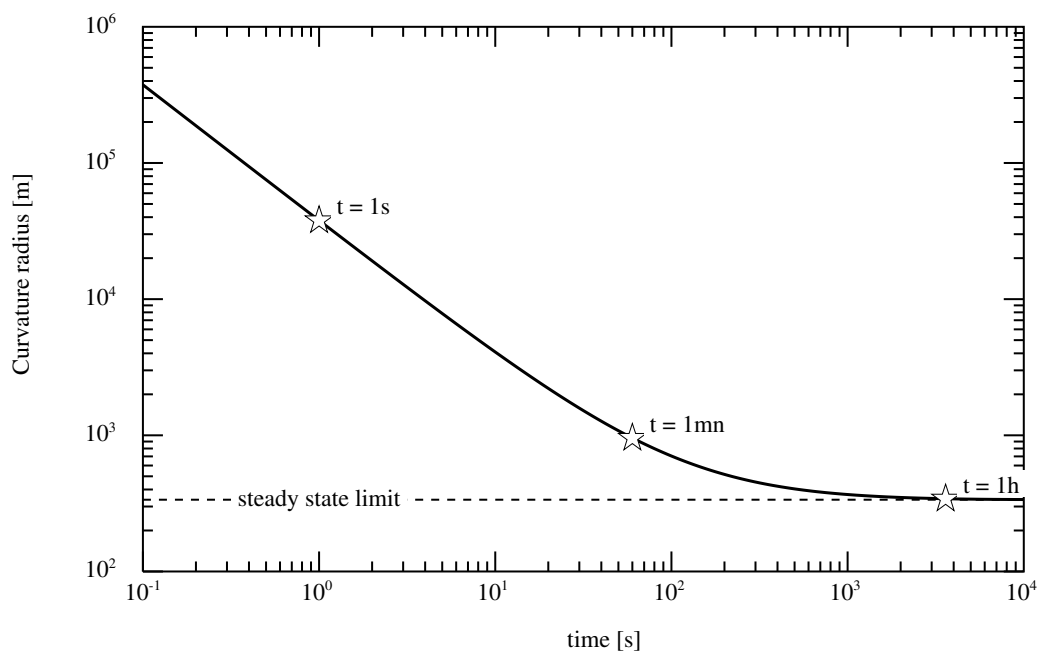

Figure 39: Coating absorption: time evolution of the curvature radius of the thermal lens $\mathrm{LG}_{0,0}$ mode, $w=2 \mathrm{~cm}$ 


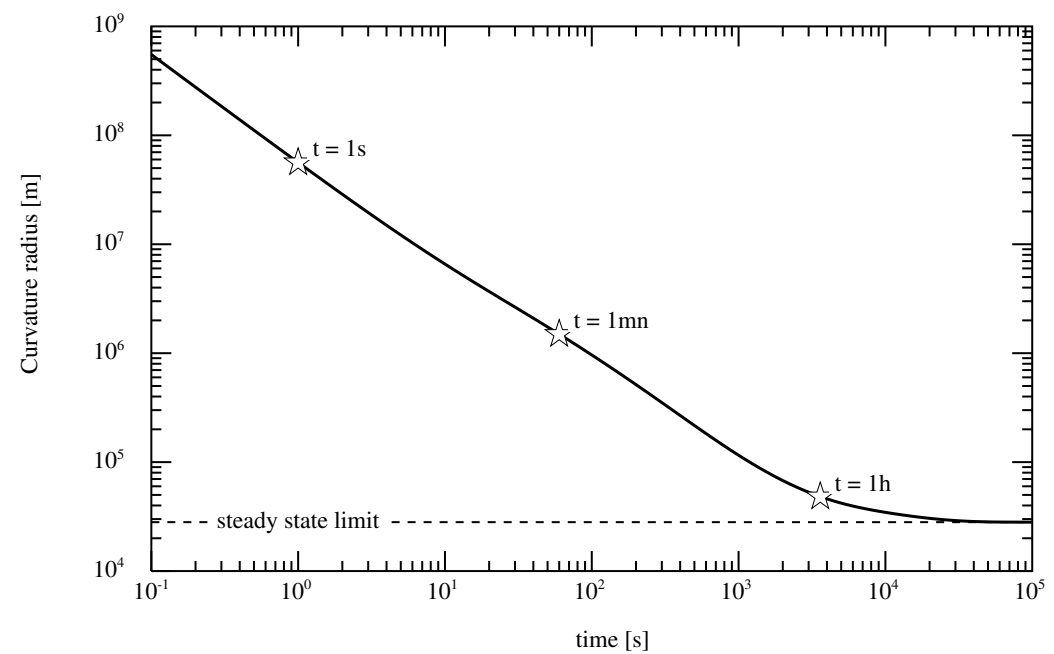

Figure 40: Coating absorption: time evolution of the curvature radius of the thermal lens, $\mathrm{LG}_{5,5}$ mode, $w=3.5 \mathrm{~cm}$

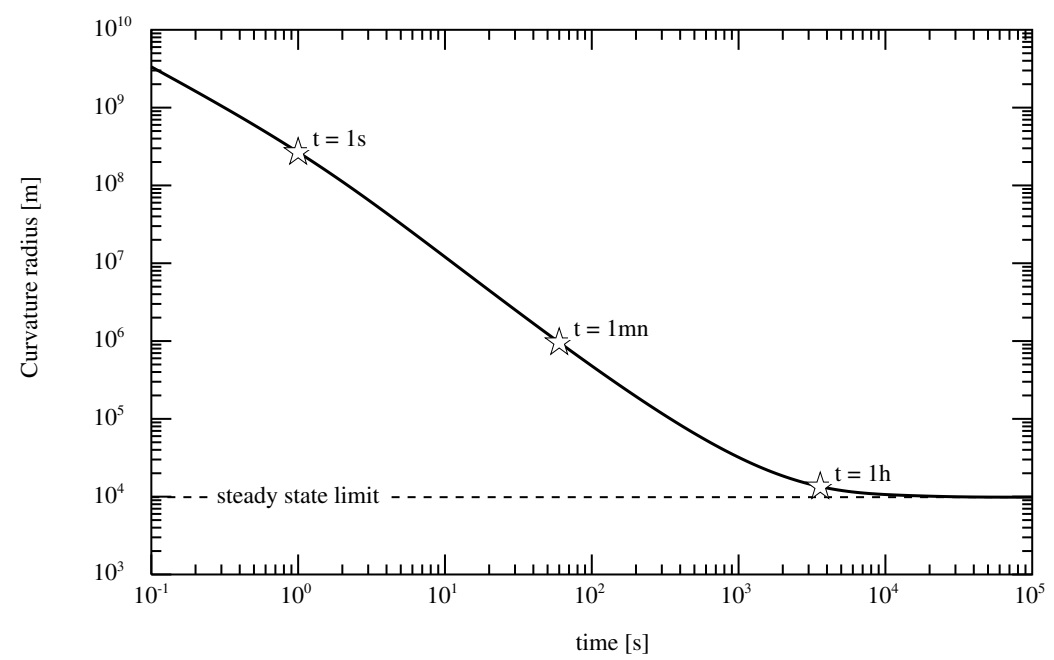

Figure 41: Coating absorption: time evolution of the curvature radius of the thermal lens, flat mode, $b=9.1 \mathrm{~cm}$ 


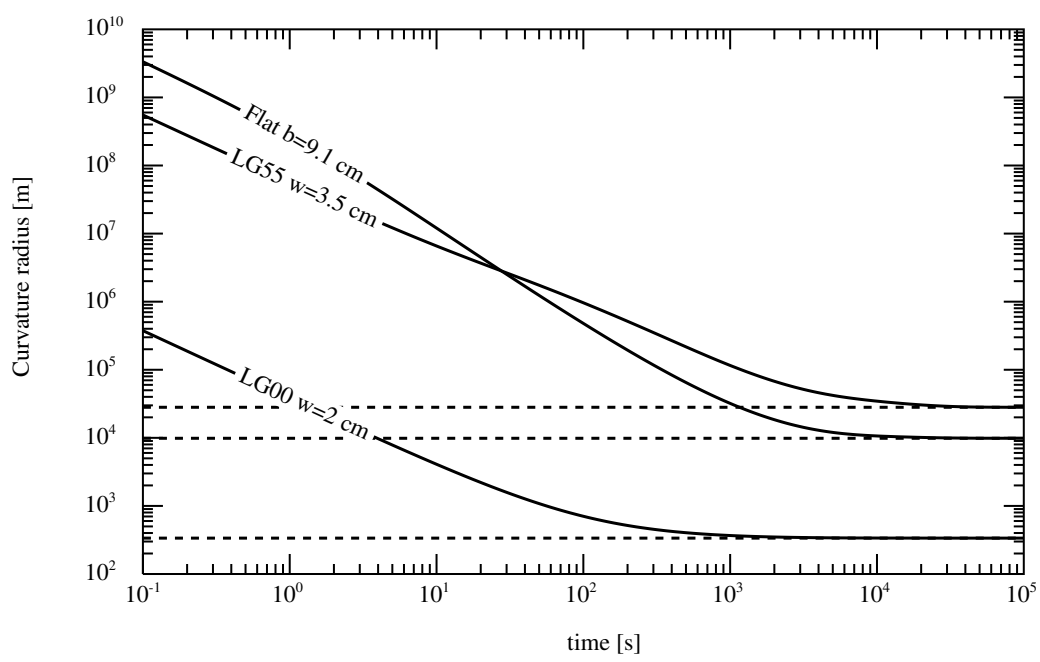

Figure 42: Coating absorption: time evolution of the curvature radii of thermal lenses for three examples

and the transient thermal lens is

$$
Z(t, r)=\frac{d n}{d T} \frac{\beta P h^{3}}{2 \pi K a^{2}} \sum_{s, j} p_{s} \frac{\left(\sin u_{j} / u_{j}\right)^{2}}{\left(1+\sin \left(2 u_{j}\right) / 2 u_{j}\right)\left(\gamma_{s}^{2}+u_{j}^{2}\right)}\left(1-\mathrm{e}^{-t / \tau_{s, j}^{\prime}}\right) J_{0}\left(k_{s} r\right) .
$$

The time evolution curves are practically identical to those shown in Figures 36, 37, and 38. See, for example, Figure 43; in the same way, the final lens profile is reached long before the temperature reaches the steady state.

\subsection{Transient thermal distortions}

In the quasistatic regime, the time scale for temperature evolution is clearly too long to generate inertial effects in the material. Thus, the elastodynamic equations reduce to elastic. The equilibrium equations are therefore unchanged with respect to Equation (3.91). The displacement vector is unchanged in form with respect to the static case, except that the time enters as an evolution parameter through temperature. The temperature field being, as usual,

$$
T(t, r, z)=\sum_{s} T_{s}(t, z) J_{0}\left(k_{s} r\right) .
$$

The generic thermoelastic longitudinal displacement is of the form

$$
u_{z}(t, r, z)=\sum_{s} B_{s}(t, z) J_{0}\left(\zeta_{s} r / a\right)
$$

We are interested in the displacement of the reflecting surface, and the general solution gives

$$
\begin{aligned}
B_{s}(t,-h / 2)= & 2 \alpha(1+\sigma)\left\{\frac{\cosh \gamma_{s}}{\Gamma_{s}^{\prime \prime}}\left[e_{s}^{\prime}(t) \sinh \gamma_{s}-k_{s} o_{s} \cosh \gamma_{s}\right]\right. \\
& \left.\frac{\sinh \gamma_{s}}{\Gamma_{s}^{\prime}}\left[o_{s}^{\prime}(t) \cosh \gamma_{s}-k_{s} e_{s} \sinh \gamma_{s}\right]\right\},
\end{aligned}
$$




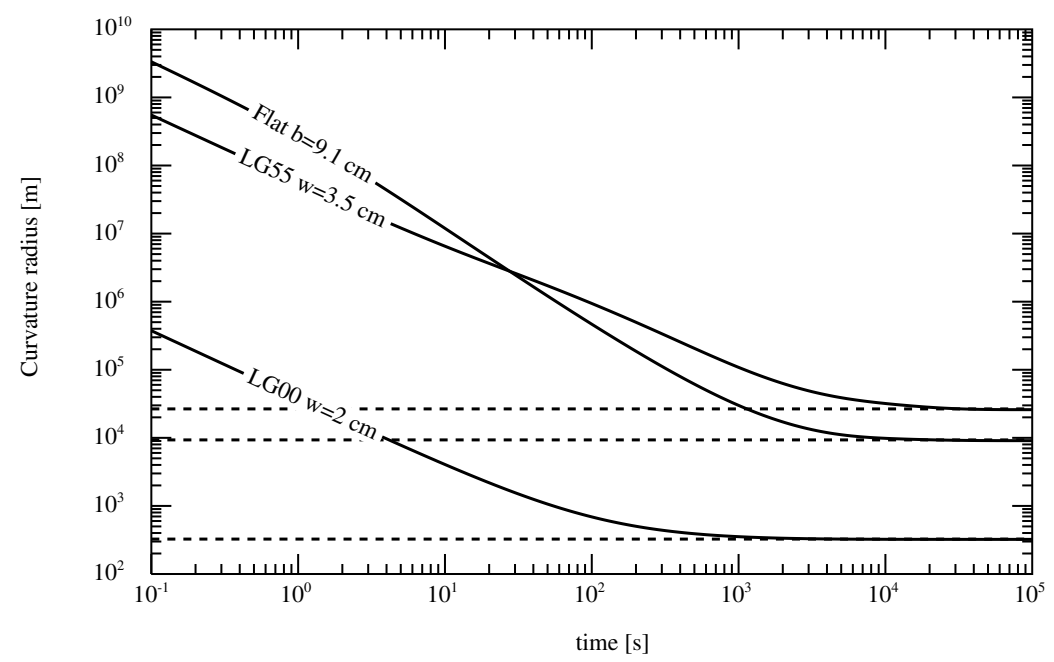

Figure 43: Bulk absorption: Time evolution of the thermal lens curvature radii for three examples

where the function $\mathcal{T}_{s}(t, z)$ is a special solution of

$$
\left(\partial_{z}^{2}-k_{s}^{2}\right) \mathcal{T}_{s}(t, z)=T_{s}(t, z)
$$

and where the notation of Equation (3.148) has been employed.

\subsubsection{Case of coating absorption}

In the case of a heat source on the coating, we have found the temperature field from Equation (5.33), so that we have

$$
\begin{aligned}
\mathcal{T}_{s}(t, z)= & -\frac{\epsilon P h^{3} p_{s}}{8 \pi K a^{2}} \sum_{j}\left[\frac{\cos u_{j}\left(1-\exp \left(-t / \tau_{s, j}^{\prime}\right)\right)}{\left(1+\sin \left(2 u_{j}\right) / 2 u_{j}\right)\left(\gamma_{s}^{2}+u_{j}^{2}\right)^{2}} \cos \left(\kappa_{j} z\right)\right. \\
& \left.-\frac{\sin v_{j}\left(1-\exp \left(-t / \tau_{s, j}^{\prime \prime}\right)\right)}{\left(1-\sin \left(2 v_{j}\right) / 2 v_{j}\right)\left(\gamma_{s}^{2}+v_{j}^{2}\right)^{2}} \sin \left(\kappa_{j} z\right)\right]
\end{aligned}
$$

and consequently

$$
\begin{gathered}
k_{s} e_{s}(t)=-\frac{\epsilon h^{3} p_{s}}{8 K a} \zeta_{s} U_{s, j}(t) \\
k_{s} o_{s}(t)=\frac{\epsilon h^{3} p_{s}}{8 K a} \zeta_{s} V_{s, j}(t) \\
e_{s}^{\prime}(t)=-\frac{\epsilon h^{3} p_{s}}{8 K a} \chi V_{s, j}(t) \\
e_{s}^{\prime}(t)=\frac{\epsilon h^{3} p_{s}}{8 K a} \chi U_{s, j}(t)
\end{gathered}
$$


with

$$
\begin{aligned}
& U_{s, j}(t)=\frac{\cos ^{2} u_{j}\left(1-\exp \left(-t / \tau_{s, j}^{\prime}\right)\right.}{\left(1+\sin \left(2 u_{j}\right) / 2 u_{j}\right)\left(\gamma_{s}^{2}+u_{j}^{2}\right)^{2}} \\
& V_{s, j}(t)=\frac{\sin ^{2} v_{j}\left(1-\exp \left(-t / \tau_{s, j}^{\prime \prime}\right)\right.}{\left(1-\sin \left(2 v_{j}\right) / 2 v_{j}\right)\left(\gamma_{s}^{2}+v_{j}^{2}\right)^{2}},
\end{aligned}
$$

so that finally

$$
B_{s}(t,-h / 2)=-\frac{\alpha(1+\sigma) \epsilon P h^{3}}{4 \pi K a^{3}} p_{s} \sum_{j}\left[\frac{d_{1, s} \sinh \gamma_{s} U_{s, j}(t)}{\Gamma_{s}^{\prime}}+\frac{d_{2, s} \cosh \gamma_{s} V_{s, j}(t)}{\Gamma_{s}^{\prime \prime}}\right] .
$$

The contribution to curvature of the Saint-Venant correction reduces to

$$
\delta u_{z}(t, r,-h / 2)=c(t) r^{2}
$$

with the time dependent curvature

$$
\begin{aligned}
c(t)= & \frac{3 \chi P \alpha \epsilon h}{2 \pi K a^{3}} \sum_{s, j} \frac{p_{s} J_{0}\left(\zeta_{s}\right)}{\zeta_{s}^{2}}\left[(\chi+2 a / h)\left(1+\gamma_{s}^{2} / v_{j}^{2}\right)\right. \\
& \left.+2 \sigma d_{2, s}\left(\sinh \gamma_{s}-\gamma_{s} \cosh \gamma_{s}\right) / \gamma_{s} \Gamma_{s}^{\prime \prime}\right] V_{s, j}(t) .
\end{aligned}
$$

See in Figures 44, 45, and 46, the time evolution of the surface deformation under a constant power flux. The dashed curves corresponding to the steady state are computed with Equations (3.120) and (3.122). Using our averaging technique, we can compute the transient curvature radius for the three considered examples (Figures 47, 48, and 49).

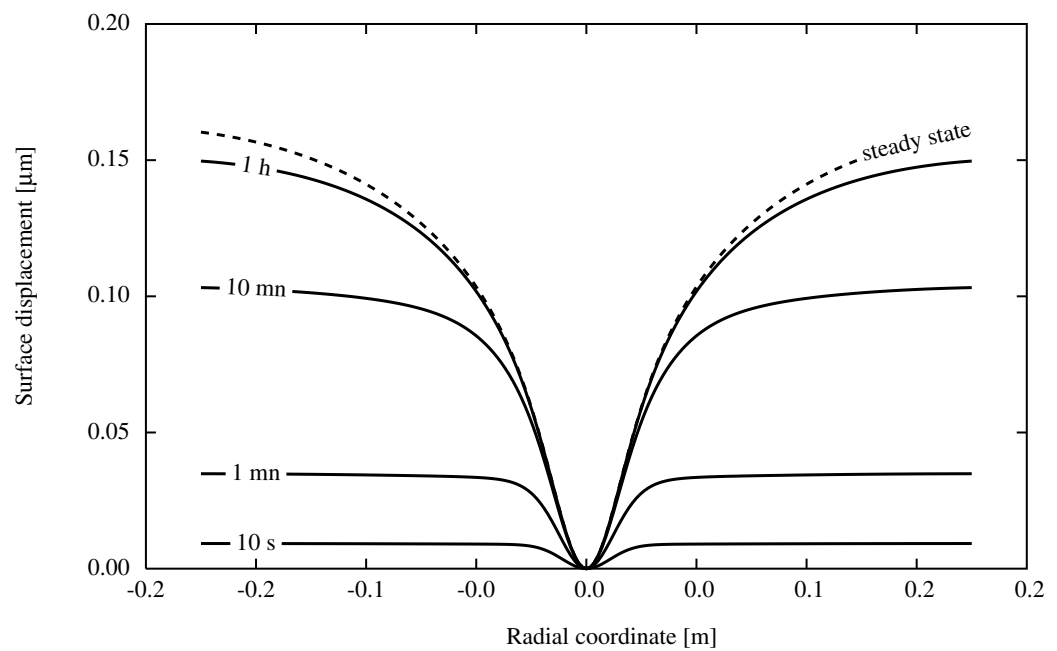

Figure 44: Coating absorption: time evolution of the reflecting surface caused by thermal expansion. Mode $\mathrm{LG}_{0,0}, w=2 \mathrm{~cm}$ 


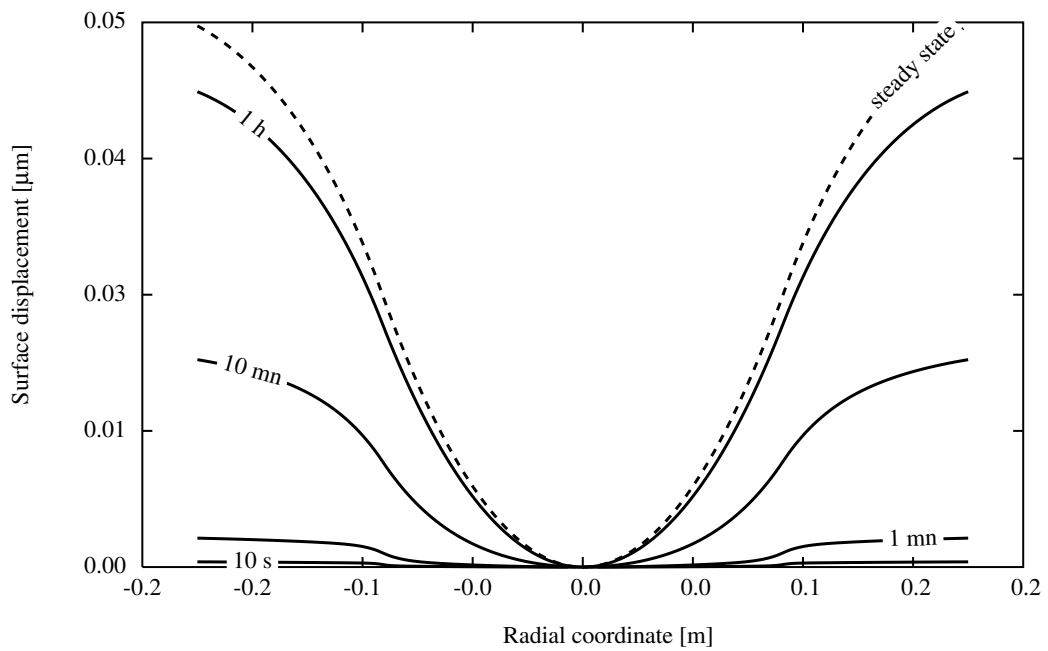

Figure 45: Coating absorption: time evolution of the reflecting surface caused by thermal expansion. Flat mode, $b=9.1 \mathrm{~cm}$

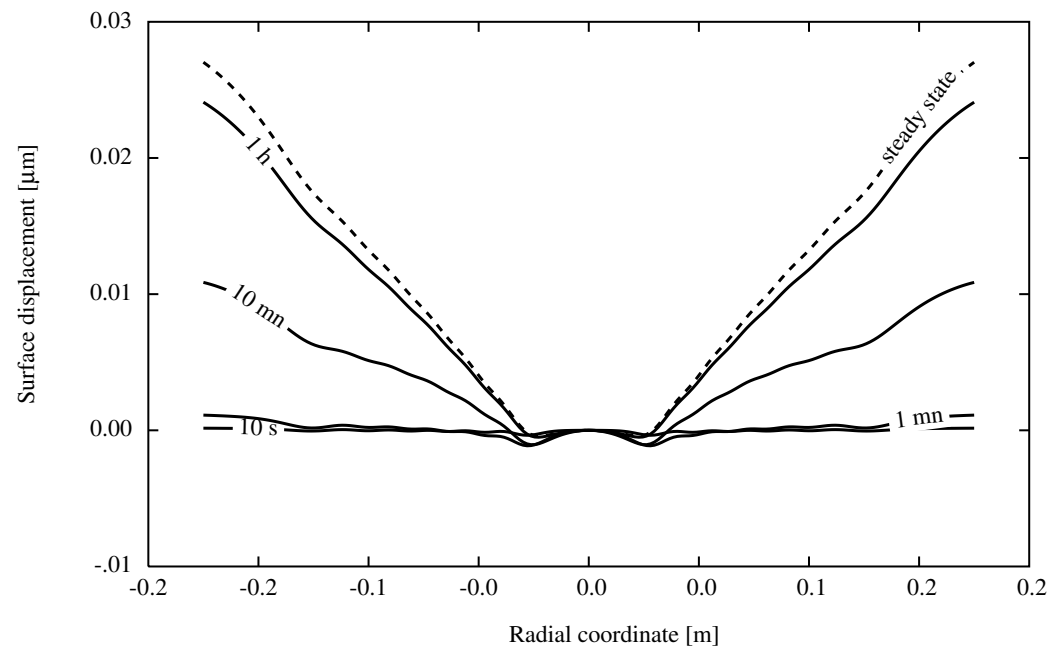

Figure 46: Coating absorption: time evolution of the reflecting surface caused by thermal expansion. Mode $\mathrm{LG}_{5,5}, w=3.5 \mathrm{~cm}$ 


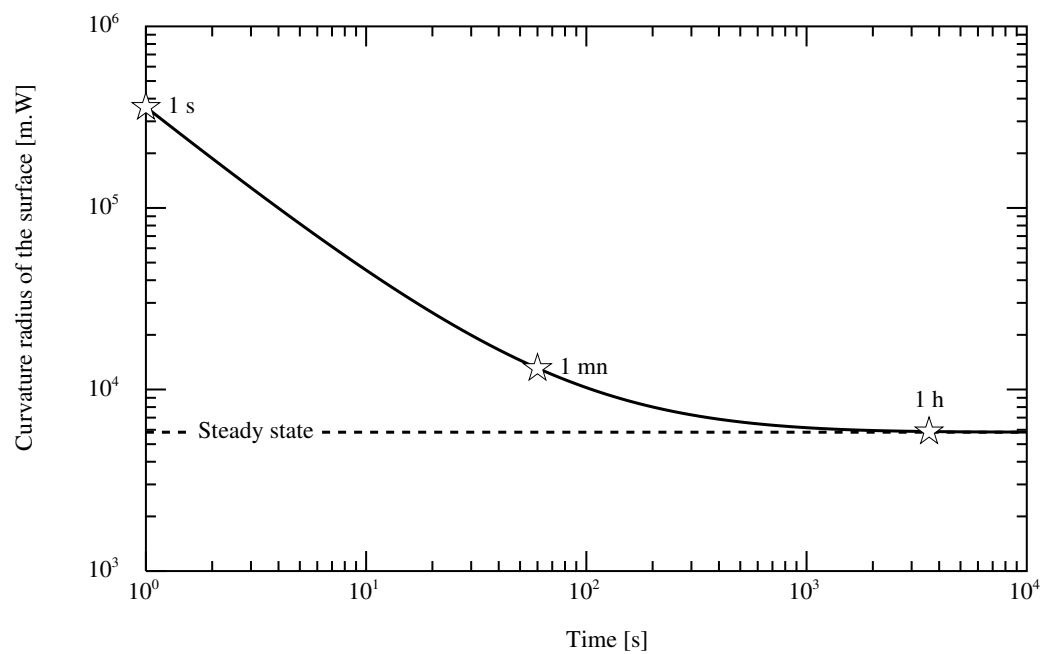

Figure 47: Coating absorption: time evolution of the curvature radius of the thermal lens caused by thermal expansion. Mode $\mathrm{LG}_{0,0}, w=2 \mathrm{~cm}$

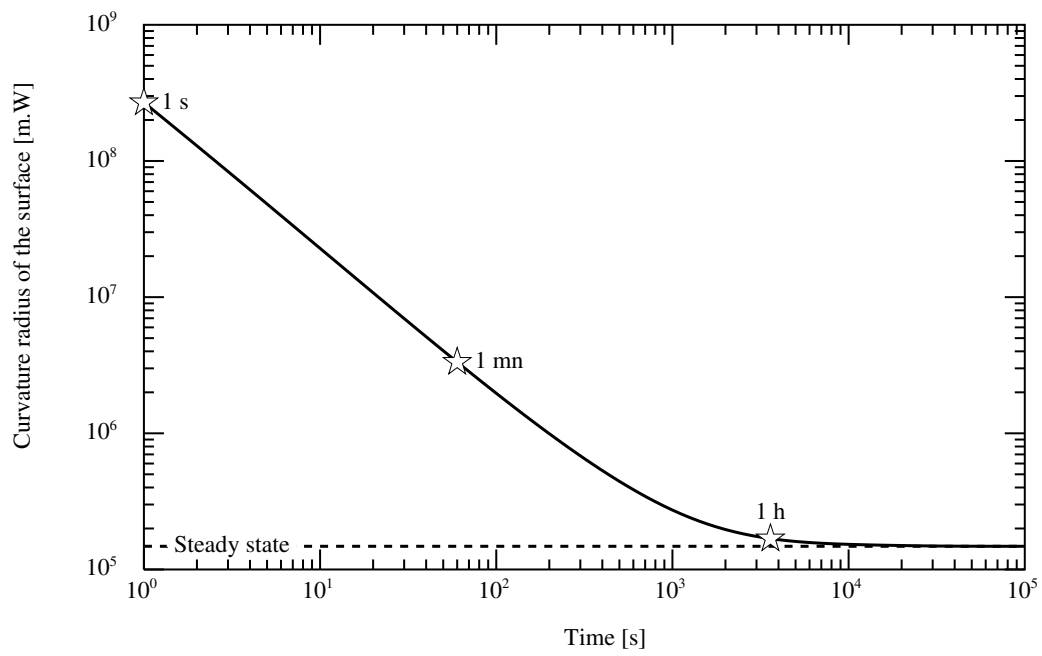

Figure 48: Coating absorption: time evolution of the curvature radius of the thermal lens caused by thermal expansion. Flat mode, $b=9.1 \mathrm{~cm}$ 


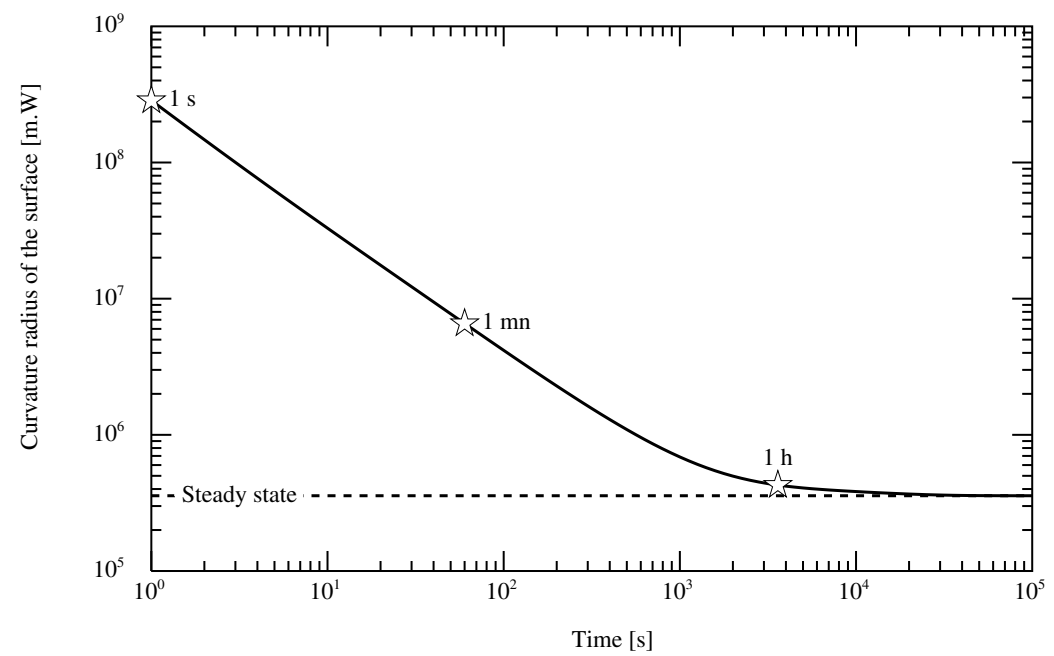

Figure 49: Coating absorption: time evolution of the curvature radius of the thermal lens caused by thermal expansion. Mode $\mathrm{LG}_{5,5}, w=3.5 \mathrm{~cm}$

\subsubsection{Case of bulk absorption}

The temperature field defined by Equation (5.37) gives

$$
\mathcal{T}_{s}(t, z)=-\frac{\beta P h^{4} p_{s}}{8 \pi K a^{2}} \sum_{j} \frac{\sin u_{j} / u_{j}}{\left(1+\sin \left(2 u_{j}\right) / 2 u_{j}\right)\left(\gamma_{s}^{2}+u_{j}^{2}\right)^{2}}\left[1-\exp \left(-t / \tau_{s, j}\right)\right] \cos \left(\kappa_{j} z\right)
$$

so that

with

$$
\begin{aligned}
k_{s} e_{s}(t) & =-\frac{\beta P h^{4}}{8 \pi K a^{3}} p_{s} \zeta_{s} W_{s, j}(t) \\
o^{\prime}(t) & =\frac{\beta P h^{3}}{8 \pi K a^{3}} p_{s} \chi W_{s, j}(t)
\end{aligned}
$$

$$
W_{s, j}(t)=\frac{\sin \left(2 u_{j}\right) / 2 u_{j}\left(1-\exp \left(-t / \tau_{s, j}^{\prime}\right)\right)}{\left(1+\sin \left(2 u_{j}\right) / 2 u_{j}\right)\left(\gamma_{s}^{2}+u_{j}^{2}\right)^{2}}
$$

yielding, finally,

$$
B_{s}(t,-h / 2)=-\frac{\alpha(1+\sigma) \beta P h^{4}}{4 \pi K a^{3}} \sum_{j} \frac{p_{s} d_{1, s} \sinh \gamma_{s}}{\Gamma_{s}^{\prime}} W_{s, j}(t)
$$

Due to the symmetry of the temperature field in $z$ and to the resulting symmetry of the stress field $\Theta_{r} r(a, z)$, the contribution to curvature of the Saint-Venant correction (mean torque) is zero. See in Figures 50, 51, and 52 the time evolution of the distorted surface for our three examples. The curvature evolution is plotted in Figures 53,54, and 55. 


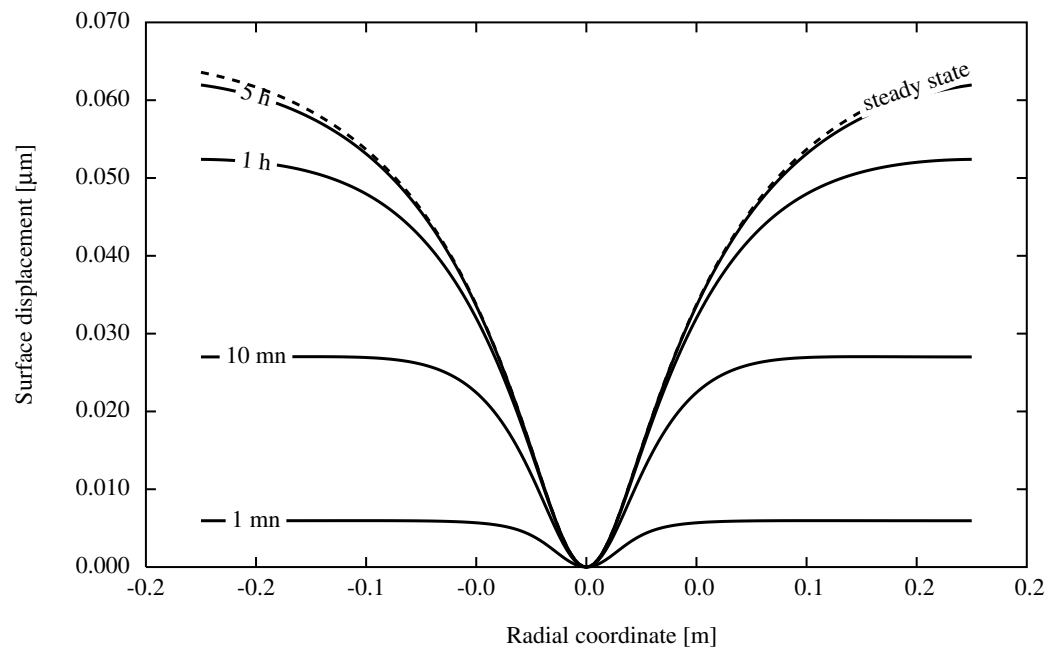

Figure 50: Bulk absorption: time evolution of the reflecting surface caused by thermal expansion. Mode $\mathrm{LG}_{0,0}, w=2 \mathrm{~cm}$

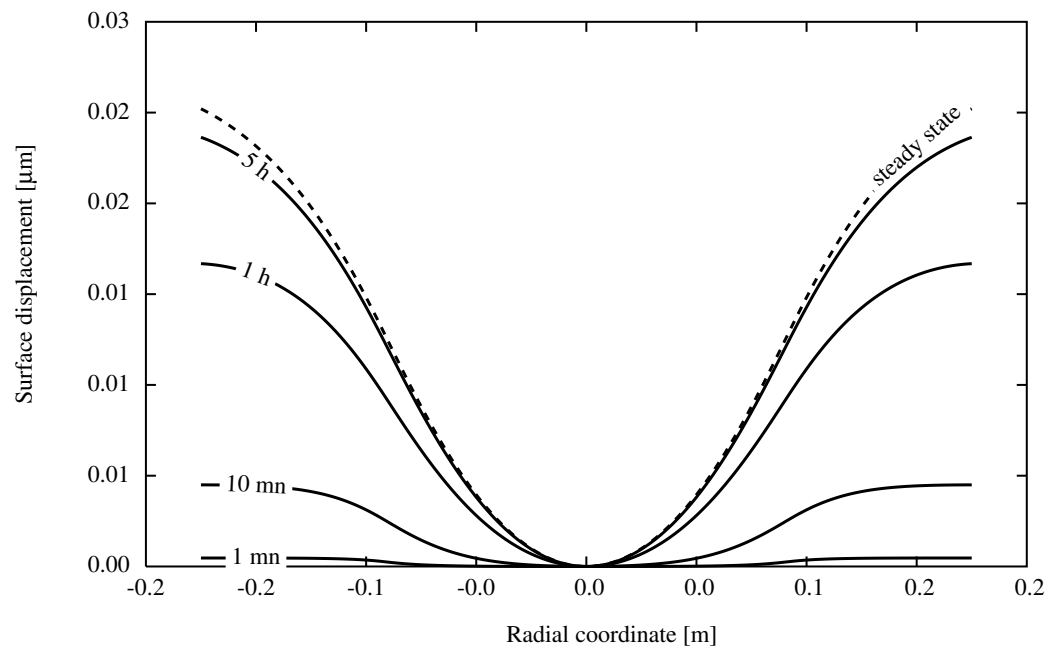

Figure 51: Bulk absorption: time evolution of the reflecting surface caused by thermal expansion. Flat mode, $b=9.1 \mathrm{~cm}$ 


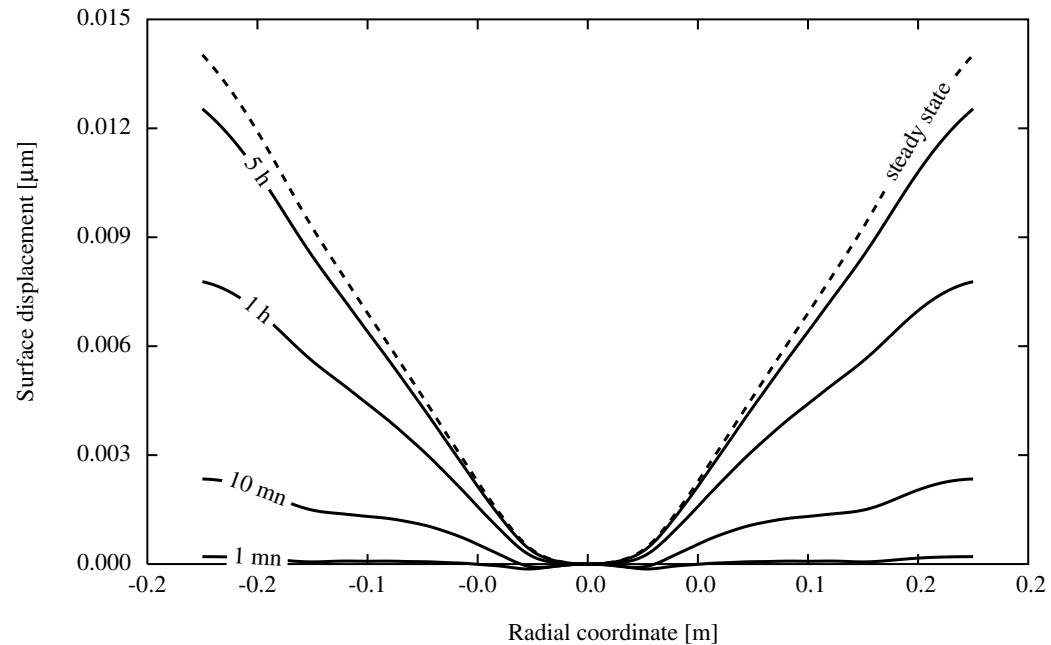

Figure 52: Bulk absorption: time evolution of the reflecting surface caused by thermal expansion. Mode $\mathrm{LG}_{5,5}, w=3.5 \mathrm{~cm}$

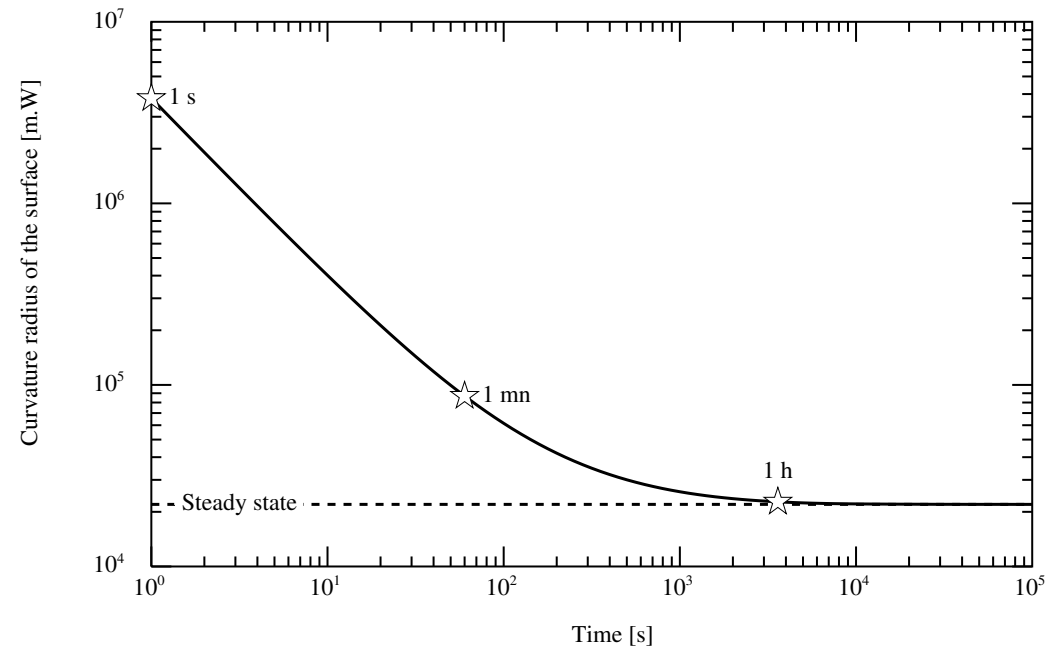

Figure 53: Bulk absorption: time evolution of the curvature radius caused by thermal expansion. Mode $\mathrm{LG}_{0,0}, w=2 \mathrm{~cm}$ 


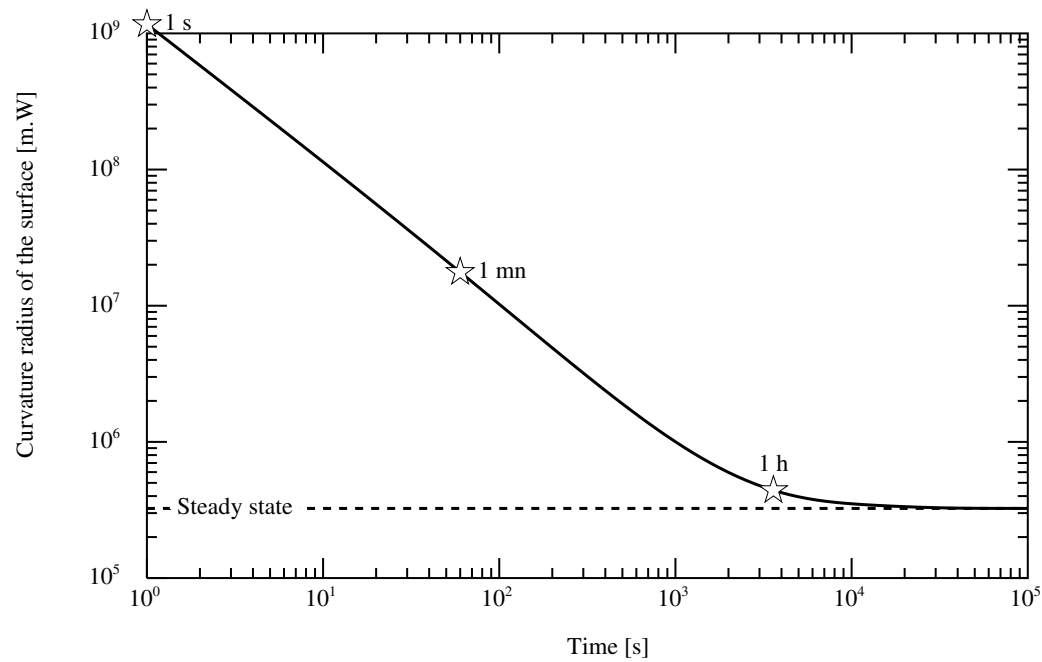

Figure 54: Bulk absorption: time evolution of the curvature radius caused by thermal expansion. Flat mode, $b=9.1 \mathrm{~cm}$

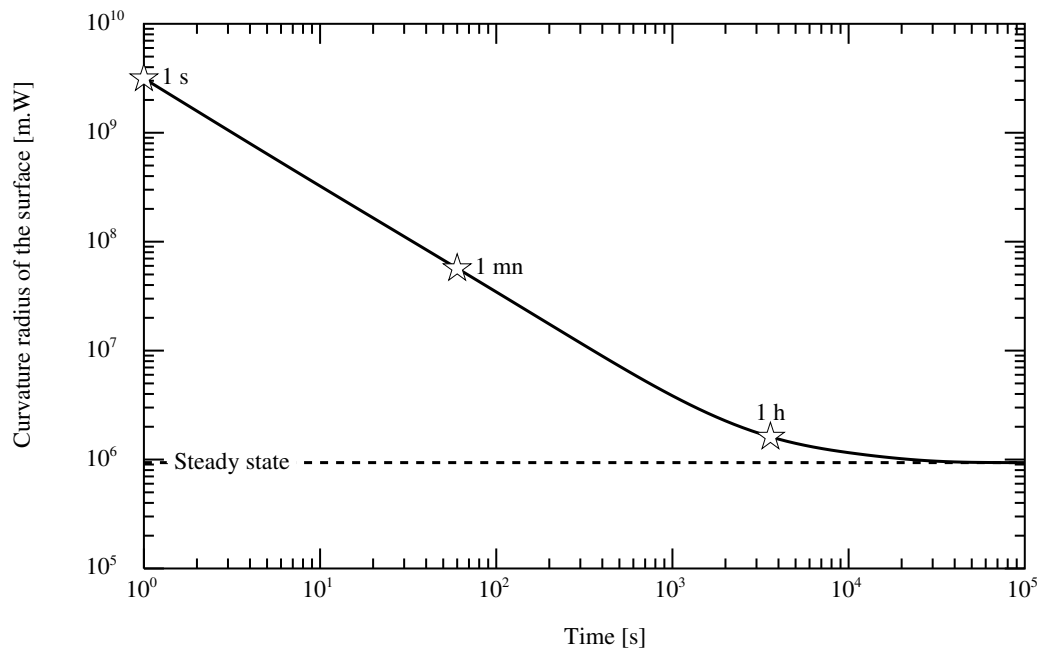

Figure 55: Bulk absorption: time evolution of the curvature radius caused by thermal expansion. Mode $\mathrm{LG}_{5,5}, w=3.5 \mathrm{~cm}$ 


\section{Heating and Thermal Effects in the Dynamic Regime: Transfer Functions}

We address here the case of fluctuations of the incident power due to either fluctuations of the laser itself, fluctuations of the locking system, or even fluctuations equivalent to shot noise. The incident power flux $P(\omega)$ is assumed to be of Fourier frequency $f=\omega / 2 \pi$. The Fourier transform of the Heat equation (assuming a time dependence of all quantities in $\exp (i \omega t)$ ) is

$$
\left[\partial_{r}^{2}+\frac{1}{r} \partial_{r}+\partial_{z}^{2}-i \frac{\rho C \omega}{K}\right] T(\omega, r, z)=0
$$

in the case of a heat source on the coating. We assume a separate solution in the form an FB expansion of the form

$$
T(\omega, r, z)=\sum_{s} T_{s}(\omega, z) J_{0}\left(k_{s} r\right)
$$

where $k_{s}$ has the usual definition $k_{s} \equiv \zeta_{s} / a$, in order to identically satisfy the radiation condition on the edge of the mirror. Then, the longitudinal function obeys

$$
\left[\partial_{z}^{2}-k_{s}^{2}-i \frac{\rho C \omega}{K}\right] T_{s}(\omega, z)=0
$$

so that the general solution is of the form

$$
T_{s}(\omega, z)=T_{s}^{(1)}(\omega) \cosh \left(\kappa_{s} z\right)+T_{s}^{(2)}(\omega) \sinh \left(\kappa_{s} z\right)
$$

with

$$
\kappa_{s}=\kappa_{s}(\omega) \equiv \sqrt{k_{s}^{2}+i \frac{\rho C \omega}{K}}
$$

We also adopt the new dimensionless parameters $\xi_{s} \equiv \kappa_{s} a \equiv \sqrt{\zeta_{s}^{2}+i \rho C \omega a^{2} / K}$ and $\eta_{s} \equiv \kappa_{s} h / 2$. With this notation, and by setting the (unchanged since the beginning) boundary conditions, we can specialize the solution to be entirely analogous in form to the static solution.

\subsection{Temperature fields and thermal lensing}

\subsubsection{Coating absorption}

In case of dissipation of light power on the reflecting surface, we find the complex solution (see Equation (3.18))

$$
T(\omega, r, z)=\frac{\epsilon P(\omega)}{2 \pi K a} \sum_{s} p_{s}\left[\frac{\cosh \left(\kappa_{s} z\right)}{\xi_{s} \sinh \eta_{s}+\chi \cosh \eta_{s}}-\frac{\sinh \left(\kappa_{s} z\right)}{\xi_{s} \cosh \eta_{s}+\chi \sinh \eta_{s}}\right] J_{0}\left(k_{s} r\right)
$$

In turn, the dynamic thermal lens is

$$
Z(\omega, r)=\frac{d n}{d T} \frac{\epsilon P(\omega)}{\pi K} \sum_{s} p_{s} \frac{1}{\xi_{s}\left(\xi_{s}+\chi \operatorname{coth} \eta_{s}\right)} J_{0}\left(k_{s} r\right)
$$




\subsubsection{Bulk absorption}

For internal dissipation, we have, in the same way,

$$
T(\omega, r, z)=\frac{\beta P(\omega)}{\pi K} \sum_{s} \frac{p_{s}}{\zeta_{s}^{2}}\left[1-\frac{\chi \cosh \left(\kappa_{s} z\right)}{\xi_{s} \sinh \eta_{s}+\chi \cosh \eta_{s}}\right] J_{0}\left(k_{s} r\right)
$$

with the resulting thermal lens

$$
Z(\omega, r)=\frac{d n}{d T} \frac{\beta h P(\omega)}{\pi K} \sum_{s} \frac{p_{s}}{\xi_{s}^{2}}\left[1-\frac{2 a \chi / h}{\xi_{s}\left(\xi_{s}+\chi \operatorname{coth} \eta_{s}\right)}\right] J_{0}\left(k_{s} r\right) .
$$

\subsection{Equivalent displacement noise}

The induced dynamic thermal lens produces a dynamic excess phase after crossing the mirror's substrate. This dynamic phase is analogous to a displacement. We assume this equivalent displacement is not desired and call it "displacement noise". This equivalent displacement $Z(\omega)$ is given, as usual, by the average of the lens, weighted by the normalized intensity profile $I(r)$,

$$
Z(\omega)=2 \pi \int_{0}^{a} Z(\omega, r) I(r) r d r
$$

We have

$$
Z(\omega, r)=\sum_{s} Z_{s}(\omega) J_{0}\left(k_{s} r\right)
$$

and

$$
I(r)=\frac{1}{\pi a^{2}} \sum_{s} p_{s} J_{0}\left(k_{s} r\right)
$$

so that the equivalent displacement is

$$
Z(\omega)=\sum_{s}\left[1+\left(\chi_{e} / \zeta_{s}\right)^{2}\right] J_{0}\left(\zeta_{s}\right)^{2} p_{s} Z_{s}(\omega)
$$

\section{Asymptotic regime}

The time constant $\tau_{d} \equiv \rho C a^{2} / K$ is about $10 \mathrm{~h}$. Therefore, it is clear that for frequencies in the target GW band (more than a few $\mathrm{Hz}$ ), we have

$$
\xi_{s}^{2}=\zeta_{s}^{2}+2 i \pi \tau_{d} f \sim 2 i \pi \tau_{d} f
$$

Because the FB series is converging, the values of $s$ at which the real part becomes comparable to the imaginary are never reached. If we adopt the preceding approximation, we have

$$
Z_{s}(\omega) \sim-i \frac{d n}{d T} \frac{\epsilon P(\omega)}{\pi \rho C \omega} p_{s}
$$

which allows one to compute the asymptotic equivalent displacement. In Figure 56 we have plotted the transfer function $|Z(\omega) / \epsilon P(\omega)|$ relating displacement fluctuations to power fluctuations, making clear that the asymptotic regime (dashed line) is fully valid for frequencies larger than $10 \mathrm{mHz}$. On the other hand, we see that in the asymptotic regime, the dynamic thermal lens is simply

$$
Z(\omega, r)=-i \frac{d n}{d T} \frac{\epsilon P(\omega)}{\pi \rho C \omega} I(r)
$$




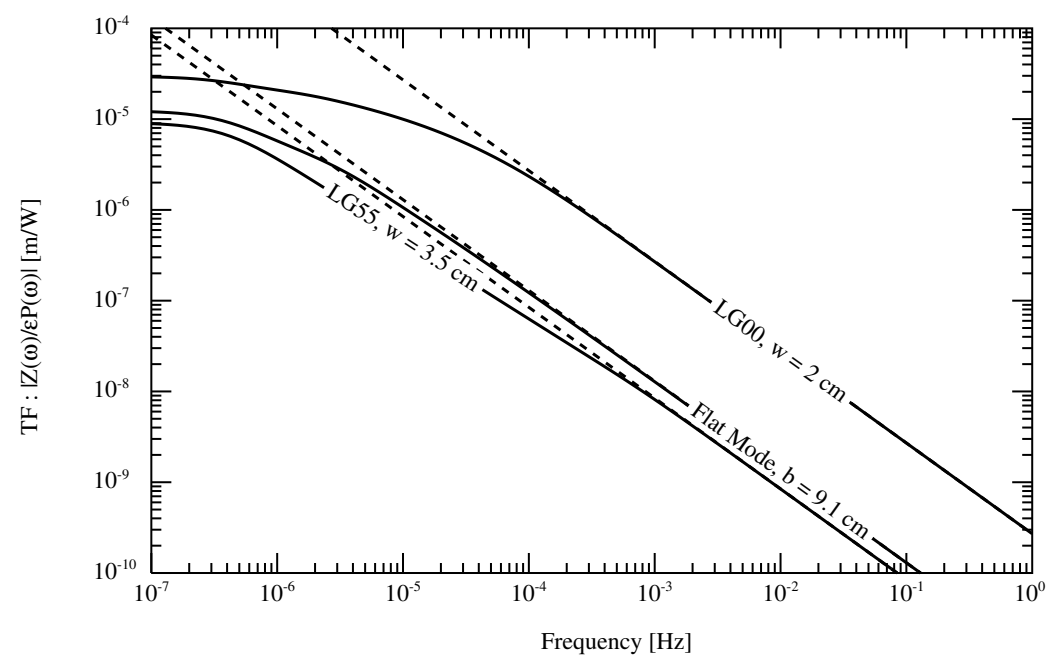

Figure 56: Coating absorption: transfer function from power to displacement. Dashed line: asymptotic regime

where $I(r)$ is the normalized intensity of the beam. In words, the thermal lens is proportional to the beam intensity, with a phase lag of $\pi / 2$. The conclusions are identical for the case of bulk absorption. The asymptotic formulas are identical up to the change $\epsilon \rightarrow \beta h$.

For the case of the heat source on the reflective coating, we get the following values. For an $\mathrm{LG}_{0,0}$ mode with $w=2 \mathrm{~cm}$, we have

$$
\frac{Z(f)}{\epsilon P(f)} \sim 2.7 \times 10^{-10}\left[\frac{1 \mathrm{~Hz}}{f}\right] \mathrm{m} / \mathrm{W}
$$

for a flat mode of width $9.1 \mathrm{~cm}$, we have

$$
\frac{Z(f)}{\epsilon P(f)} \sim 1.3 \times 10^{-11}\left[\frac{1 \mathrm{~Hz}}{f}\right] \mathrm{m} / \mathrm{W} ;
$$

and for an $\mathrm{LG}_{5,5}$ mode with $w=3.5 \mathrm{~cm}$, we have

$$
\frac{Z(f)}{\epsilon P(f)} \sim 8.5 \times 10^{-12}\left[\frac{1 \mathrm{~Hz}}{f}\right] \mathrm{m} / \mathrm{W} .
$$

The results are quasi-identical for bulk absorption (see Figure 57). 


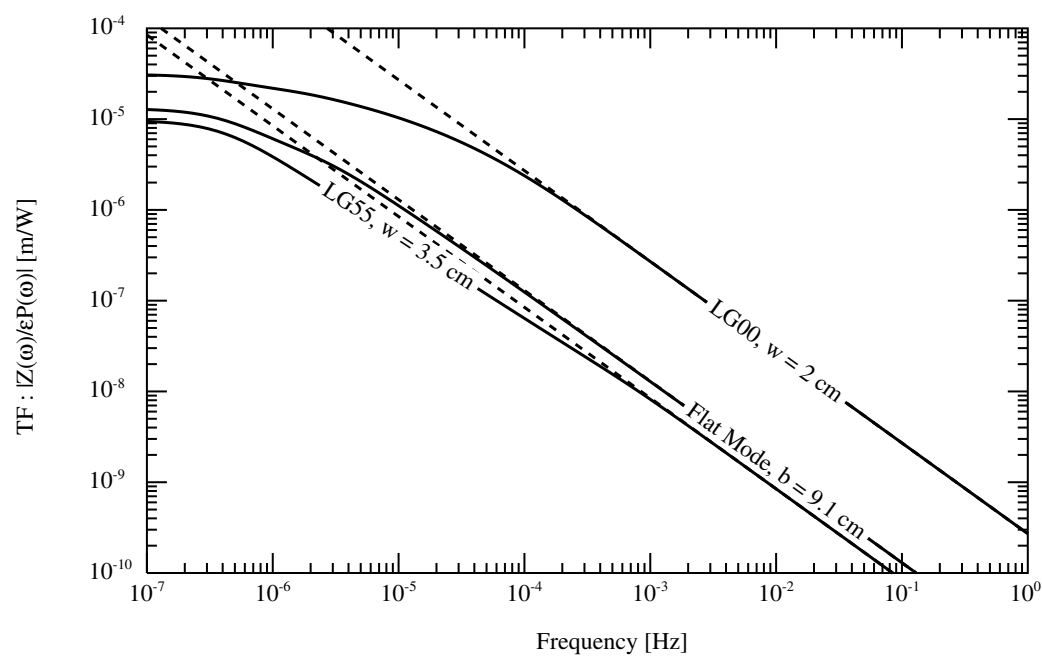

Figure 57: Bulk absorption: transfer function from power to displacement. Dashed line: asymptotic regime.

\section{Dynamic Surface Distortion}

We address here the motion of the reflecting surface of a mirror receiving a time-varying power flow creating a time-varying temperature and finally time-varying stresses. For the temperature field, the problem was solved in the preceding Section 6. We take the generic form

$$
T(\omega, r, z)=\sum_{s} T_{s}(\omega, z) J_{0}\left(k_{s} r\right)
$$

And we search for a displacement vector in the (already used) form

$$
\left\{\begin{array}{l}
u_{r}(\omega, r, z)=\sum_{s} A_{s}(\omega, z) J_{1}\left(k_{s} r\right) \\
u_{s}(\omega, r, z)=\sum_{s} B_{s}(\omega, z) J_{0}\left(k_{s} r\right)
\end{array}\right.
$$

with, as usual, $k_{s} \equiv \zeta_{s} / a$. The equilibrium equations must be modified with respect to Equation (3.91) to take into account inertial effects

$$
\left\{\begin{array}{l}
\partial_{r} \Theta_{r r}+\left(\Theta_{r r}-\Theta_{\phi \phi}\right) / r+\partial_{z} \Theta_{r z}=-\rho \omega^{2} u_{r} \\
\left(\partial_{r}+1 / r\right) \Theta_{r z}+\partial_{z} \Theta_{z z}=-\rho \omega^{2} u_{z}
\end{array}\right.
$$

with the expression of the displacement vector, and the expression of thermoelastic stresses, this gives

$$
\begin{gathered}
\mu\left[\partial_{z}^{2}-k_{s}^{2}+\frac{\rho \omega^{2}}{\mu}\right] A_{s}-(\lambda+\mu) k_{s}\left(\partial_{z} B_{s}+k_{s} A_{s}\right)+k_{s} \nu T_{s}=0 \\
\mu\left[\partial_{z}^{2}-k_{s}^{2}+\frac{\rho \omega^{2}}{\mu}\right] B_{s}+(\lambda+\mu) \partial_{z}\left(\partial_{z} B_{s}+k_{s} A_{s}\right)-\nu \partial_{z} T_{s}=0 .
\end{gathered}
$$

By taking $\partial_{z}(7.4)+k_{s}(7.5)$, we get

$$
\left[\partial_{z}^{2}-k_{s}^{2}+\frac{\rho \omega^{2}}{\mu}\right]\left(\partial_{z} A_{s}+k_{s} B_{s}\right) .
$$


The general solution of which is

$$
\partial_{z} A_{s}+k_{s} B_{s}=k_{s} C_{s} \cosh \left(\kappa_{T, s} z\right)+k_{s} D_{s} \sinh \left(\kappa_{T, s} z\right),
$$

where $C_{s}, D_{s}$ are arbitrary constants, and where the transverse wave number $\kappa_{T, s}$ is defined as

$$
\kappa_{T, s}=\sqrt{\left(\frac{\zeta_{s}}{a}\right)^{2}-\frac{\rho \omega^{2}}{\mu}}
$$

. Equation (7.4) can then be written as

$$
\left[\partial_{z}^{2}-k_{s}^{2}+\frac{\rho \omega^{2}}{\lambda+2 \mu}\right] A_{s}=\frac{\lambda+\mu}{\lambda+2 \mu} \partial_{z}\left(\partial_{z} A_{s}+k_{s} B_{s}\right)-k_{s} \frac{\nu}{\lambda+\mu} T_{s},
$$

which allows one to find $A_{s}$ :

$$
\begin{aligned}
A_{s}(\omega, z)= & M_{s} \sinh \left(\kappa_{L, s} z\right)+N_{s} \cosh \left(\kappa_{L, s} z\right) \\
& +\frac{\lambda+\mu}{\lambda+2 \mu} \frac{k_{s} \kappa_{T, s}}{\kappa_{T, s}^{2}-\kappa_{L, s}^{2}}\left[C_{s} \sinh \left(\kappa_{T, s} z\right)+D_{s} \cosh \left(\kappa_{T, s} z\right)\right]-k_{s} \frac{\nu}{\lambda+\mu} \mathcal{T}_{s},
\end{aligned}
$$

where $M_{s}$ and $N_{s}$ are two more arbitrary constants, $\mathcal{T}_{s}$ is a special solution of

$$
\left[\partial_{z}^{2}-\kappa_{L, s}^{2}\right] \mathcal{T}_{s}=T_{s}
$$

and the longitudinal wave number $\kappa_{L, s}$ is defined as

$$
\kappa_{L, s}=\sqrt{\left(\frac{\zeta_{s}}{a}\right)^{2}-\frac{\rho \omega^{2}}{\lambda+2 \mu}}
$$

Once $A_{s}$ is found, one obtains $B_{s}$ :

$$
\begin{aligned}
B_{s}(\omega, z)= & \left(1-\frac{\lambda+\mu}{\lambda+2 \mu} \frac{\kappa_{T, s}^{2}}{\kappa_{T, s}^{2}-\kappa_{L, s}^{2}}\right)\left[C_{s} \cosh \left(\kappa_{T, s} z\right)+D_{s} \sinh \left(\kappa_{T, s} z\right)\right] \\
& -\frac{\kappa_{L, s}}{k_{s}}\left(M_{s} \cosh \left(\kappa_{L, s} z\right)+N_{s} \sinh \left(\kappa_{L, s} z\right)\right)+\frac{\nu}{\lambda+\mu} \partial_{z} \mathcal{T}_{s}
\end{aligned}
$$

The boundary conditions on the faces allow one to determine the arbitrary constants. It is convenient to introduce the dimensionless parameter

$$
x_{s} \equiv \frac{\rho \omega^{2} a^{2}(1+\sigma)}{Y \zeta_{s}^{2}} .
$$

Then, we have

$$
\begin{aligned}
& C_{s}=\alpha \frac{1+\sigma}{1-\sigma} \frac{2 x_{s}}{D_{1, s}}\left[\left(1-x_{s}\right) \sinh \phi_{L, s} e_{s}^{\prime}+\frac{\kappa_{L, s}}{k_{s}} \cosh \phi_{L, s} k_{s} o_{s}\right] \\
& D_{s}=\alpha \frac{1+\sigma}{1-\sigma} \frac{2 x_{s}}{D_{2, s}}\left[\left(1-x_{s}\right) \cosh \phi_{L, s} o_{s}^{\prime}+\frac{\kappa_{L, s}}{k_{s}} \sinh \phi_{L, s} k_{s} e_{s}\right] \\
& M_{s}=\alpha \frac{1+\sigma}{1-\sigma} \frac{1}{D_{1, s}}\left[\left(1-x_{s}\right) \cosh \phi_{T, s} k_{s} o_{s}+\frac{\kappa_{T, s}}{k_{s}} \sinh \phi_{T, s} e_{s}^{\prime}\right]
\end{aligned}
$$




$$
N_{s}=\alpha \frac{1+\sigma}{1-\sigma} \frac{1}{D_{2, s}}\left[\left(1-x_{s}\right) \sinh \phi_{T, s} k_{s} e_{s}+\frac{\kappa_{T, s}}{k_{s}} \cosh \phi_{T, s} o_{s}^{\prime}\right]
$$

with

$$
\begin{aligned}
D_{1, s} & =\frac{\kappa_{L, s} \kappa_{T, s}}{k_{s}^{2}} \sinh \phi_{T, s} \cosh \phi_{L, s}-\left(1-x_{s}\right)^{2} \sinh \phi_{L, s} \cosh \phi_{T, s} \\
D_{2, s} & =\frac{\kappa_{L, s} \kappa_{T, s}}{k_{s}^{2}} \sinh \phi_{L, s} \cosh \phi_{T, s}-\left(1-x_{s}\right)^{2} \sinh \phi_{T, s} \cosh \phi_{L, s}
\end{aligned}
$$

and

$$
\phi_{L, s}=\kappa_{L, s} h / 2, \quad \phi_{T, s}=\kappa_{T, s} h / 2 .
$$

$e_{s}, e^{\prime} s, o_{s}, o_{s}^{\prime}$ are defined as in Equation (3.148). The surface distortion is given by

$$
Z(\omega, r)=\sum_{s} B_{s}(\omega) J_{0}\left(k_{s} r\right)
$$

and we have

$$
\begin{aligned}
B_{s}(\omega,-h / 2)= & \alpha \frac{1+\sigma}{1-\sigma} x_{s}\left[\cosh \phi_{T, s} \frac{\left(1-x_{s}\right) \sinh \phi_{L, s} e_{s}^{\prime}+\frac{\kappa_{L, s}}{k_{s}} \cosh \phi_{L, s} k_{s} o_{s}}{D_{1, s}}\right. \\
& \left.-\sinh \phi_{T, s} \frac{\left(1-x_{s}\right) \cosh \phi_{L, s} o_{s}^{\prime}+\frac{\kappa_{L, s}}{k_{s}} \sinh \phi_{L, s} k_{s} e_{s}}{D_{2, s}}\right]
\end{aligned}
$$

We have seen that the dynamic temperature field at frequencies within the GW band is practically proportional to the beam intensity profile, and consequently negligible on the edge. This is why we neglect the boundary conditions for the edge stresses here. At this point, we can specialize for the two cases of coating/bulk heating.

\subsection{Dynamic surface distortion caused by coating absorption}

The temperature field is known from Section 6 (see Equation (6.6)), so that we get

$$
\begin{gathered}
e_{s}=\frac{\epsilon P(\omega) p_{s}}{2 \pi K a} \frac{1}{\kappa_{s}^{2}-\kappa_{L, s}^{2}} \frac{1}{\xi_{s} \tanh \eta_{s}+\chi} \\
o_{s}=-\frac{\epsilon P(\omega) p_{s}}{2 \pi K a} \frac{1}{\kappa_{s}^{2}-\kappa_{L, s}^{2}} \frac{1}{\xi_{s} \operatorname{coth} \eta_{s}+\chi} \\
e_{s}^{\prime}=-\frac{\epsilon P(\omega) p_{s}}{2 \pi K a} \frac{\kappa}{\kappa_{s}^{2}-\kappa_{L, s}^{2}} \frac{1}{\xi_{s}+\chi \tanh \eta_{s}} \\
o_{s}^{\prime}=\frac{\epsilon P(\omega) p_{s}}{2 \pi K a} \frac{\kappa}{\kappa_{s}^{2}-\kappa_{L, s}^{2}} \frac{1}{\xi_{s}+\chi \operatorname{coth} \eta_{s}},
\end{gathered}
$$

which, with Equation (7.23), gives the complete solution. 


\subsubsection{Mean displacement}

We find the contribution to phase noise by computing, as usual, the mean equivalent displacement by

$$
Z(\omega)=2 \pi \int_{0}^{a} u_{z}(\omega, r,-h / 2) I(r) r d r .
$$

$\mathrm{I}(\mathrm{r})$ being the normalized intensity profile of the beam. This yields

$$
Z(\omega)=\sum_{s} B_{s}(\omega,-h / 2) p_{s}\left[1+\left(\chi_{\mathrm{e}} / \zeta_{s}\right)^{2}\right] J_{0}^{2}\left(\zeta_{s}\right)
$$

\subsubsection{Under cutoff regime}

The dimensionless parameter $x_{s}$ is very small. With the current parameters, $\zeta_{1}$ is slightly larger than one, so that

$$
\forall s x_{s}<4.3 \times 10^{-8}\left[\frac{f}{1 \mathrm{~Hz}}\right]^{2},
$$

so that in the GW band (far from mirror resonances), we can take the first-order approximation of the precedent functions with respect to $x_{s}$. The elastic wave regime begins when the frequency exceeds a value (cutoff) such that some $\kappa_{L, s}$ and $\kappa_{T, s}$ become imaginary. A study of the resonance modes can then be addressed, but this requires a careful treatment of the boundary conditions on the edge. We consider here only the case where the frequency is below the cutoff, so that a simple theory neglecting the edge is relevant and $x_{s}$ may be considered small. In particular, we find from $0.1 \mathrm{~Hz}$ to $1 \mathrm{kHz}$

$$
B_{s}(\omega,-h / 2)=-i \frac{\alpha(1+\sigma) \epsilon P(\omega)}{\pi \rho C a^{2} \omega}\left(\frac{2 \sinh ^{2} \gamma_{s} \cosh ^{2} \gamma_{s} \cdot p n g}{\sinh ^{2} \gamma_{s} \cosh ^{2} \gamma_{s}-\gamma_{s}^{2}}\right) .
$$

This gives for our three examples the following transfer functions for the displacement noise. $\mathrm{LG}_{0,0}$ mode $w=2 \mathrm{~cm}$ :

$$
|Z(\omega) / \epsilon P(\omega)|=2 \times 10^{-11} \mathrm{~m} / \mathrm{W}\left[\frac{1 \mathrm{~Hz}}{f}\right] .
$$

Flat mode $(b=9.1 \mathrm{~cm})$ or mesa mode $\left(b_{f}=10.7 \mathrm{~cm}\right)$ :

$$
|Z(\omega) / \epsilon P(\omega)|=1.2 \times 10^{-11} \mathrm{~m} / \mathrm{W}\left[\frac{1 \mathrm{~Hz}}{f}\right] .
$$

$\mathrm{LG}_{5,5}$ mode $w=3.5 \mathrm{~cm}$ :

$$
|Z(\omega) / \epsilon P(\omega)|=1.1 \times 10^{-11} \mathrm{~m} / \mathrm{W}\left[\frac{1 \mathrm{~Hz}}{f}\right] .
$$

Below $0.1 \mathrm{~Hz}$ we are in the regime where the displacements are corrected by the locking system. Above $1 \mathrm{kHz}$ the displacement noise is negligible compared to shot noise and that in the present situation. 


\section{Brownian Thermal Noise}

Brownian thermal noise is the phase noise caused at nonzero temperature by random motions of the reflecting faces of mirrors in a GW interferometer. A reflecting face can move either because it is displaced by its suspension system or because it undergoes internal stresses. At finite temperature the two effects are possible. We address here the internal stresses. Consider a massive body at temperature $T$. If $T>0$, the atoms constituting the body are excited and have random motions around their equilibrium position. The fact that they are strongly coupled to neighboring atoms makes possible the propagation of elastic waves of various types, reflecting on the faces, and the onset of stationary waves. One can show that, for a finite body (e.g., a cylinder of silica), there is a discrete infinity of such stationary waves, each corresponding to a particular elastic normal mode. At thermal equilibrium, the state of the body can be represented by a linear superposition of all the modes, with random relative phases, and, due to the energy equipartition theorem, the same energy $k_{B} T$ ( $k_{B}$ is the Boltzmann constant). The motion of atoms near a limiting surface of the body will modify its shape slightly, and, if we consider the reflecting face of a mirror, a surface distortion is a possible cause of phase change in the reflected beam, in other words, of a noise. Estimation of the resulting spectral density of phase noise is the internal thermal noise problem in massive mirrors.

\subsection{The Fluctuation-Dissipation theorem and Levin's generalized coor- dinate method}

We are interested in the spectral density of thermal noise. There is a general derivation of this spectral density, based on the Fluctuation-Dissipation (FD) theorem of Callen and Welton [9]. For an elementary dynamic system described by a degree of freedom $x$ and any driving force $F$, one can consider the resulting velocity $\tilde{v}=i \omega \tilde{x}$, and compute a mechanical impedance as $\mathcal{Z}=\tilde{v} / \tilde{F}$. Then, the power spectral density of displacement is (this is the FD theorem)

$$
S_{x}(f)=\frac{4 k_{B} T}{\omega^{2}} \Re e[\mathcal{Z}] .
$$

We can now address the problem of internal degrees of freedom in the mirrors. Internal elastic waves eventually distort the reflecting surface, causing a phase noise. We have already discussed how to obtain the information on the surface relevant to the beam. Let $u_{z}(t, x, y)$ be the $z$ component of the displacement vector of matter at the surface of the mirror. The equivalent displacement (generalized coordinate $x$ ) is, as usual, the axial displacement, averaged by the intensity profile,

$$
Z(t)=\iint u_{z}(t, x, y) I(x, y) d x d y
$$

where $I(x, y)$ is the normalized light-intensity distribution in the readout beam. We now follow the method proposed by Levin [26]. Let $F(t)$ be the corresponding driving force. The interaction energy is

$$
\mathcal{E}=-F(t) Z(t)
$$

or

$$
\mathcal{E}=\iint u_{z}(t, x, y) F(t) I(x, y) d x d y,
$$

where the displacement $u$ may be thought of as being caused by the pressure distribution $F \times I$. We address now the case of low frequencies. This case is very relevant, because resonances of mirrors are at relatively high frequencies (several $\mathrm{kHz}$ ) and the region where internal thermal noise is disturbing lies long before the first resonance, in the low frequency regime. Thus, although a

Living Reviews in Relativity

http: //www . livingreviews . org/lrr-2009-5 
general knowledge of internal thermal noise is useful, it is nevertheless extremely interesting to have the low frequency tail. This can be obtained as follows. If we consider a force $F(t)=F \mathrm{e}^{i \omega t}$ oscillating at very low frequency, the frequency will be lower than the cutoff for any standing waves. The pressure $F \times I$ will produce an oscillating stationary displacement $u$, of the form

$$
u_{z}(t, x, y)=\mathrm{e}^{i(\omega t-\phi)} u(x, y)
$$

This is equivalent to neglecting inertial forces in the motion of matter. The phase $\phi$ represents a retardation effect that dissipation may cause; in the case of thermoelastic dissipation, we know that $\phi$ can be considered very small and independent of the frequency (at least in the GW detection band). In the Fourier domain, this is

$$
u_{z}(\omega, x, y)=(1-i \phi) u_{z}(x, y)
$$

The impedance is

$$
\mathcal{Z}(f)=i \omega \frac{(1-i \phi) \iint u_{z}(x, y) I(x, y) d x d y}{F}
$$

so that

$$
\Re e[\mathcal{Z}]=\omega \phi \frac{\iint u_{z}(x, y) F \times I(x, y) d x d y}{F^{2}}
$$

, where the numerator of the fraction appears as the elastic energy stored in the solid stressed by the pressure distribution $F \times I$. The strain energy is defined in classical elasticity theory by

$$
W=\frac{1}{2} \iint u_{z}(x, y) p(x, y) d x d y
$$

where $p(x, y)$ is the pressure distribution causing the displacement $u_{z}(x, y)$ at the surface on which it is applied. Thus, we can write for the spectral density of displacement,

$$
S_{x}(f)=\frac{4 k_{B} T}{\pi f} \phi \frac{W}{F^{2}} .
$$

In fact, $W$ is proportional to $F^{2}$, so that $U \equiv W / F^{2}$ is the strain energy for a static pressure normalized to $1 \mathrm{~N}$. The spectral density of displacement takes the general (low frequency) form

$$
S_{x}(f)=\frac{4 k_{B} T}{\pi f} \phi U .
$$

The problem is reduced to the computation of $U$ and this is the main point of Levin's approach. This can be difficult in the general case of an arbitrary solid, but numerical finite-element codes are able to give more-or-less accurate estimates. However, it is possible to obtain analytic solutions in the case of axisymmetry.

\subsection{Infinite mirrors noise in the substrate}

We use the linear elasticity theory already summarized by Equations (3.87) and (3.91). A first approach, outlined in [5], consists in neglecting edge effects and treating the mirror as a plane interface between vacuum and material with no limiting transverse dimension. In this case, the elastic equilibrium equations (3.91) are satisfied by a displacement vector of the form [5]

$$
\left\{\begin{array}{l}
u_{r}(r, z)=\left(\alpha-\frac{\lambda+2 \mu}{\lambda+\mu} \beta+\beta k z\right) \mathrm{e}^{-k z} J_{1}(k r) \\
u_{z}(r, z)=\left(\alpha+\frac{\mu}{\lambda+\mu} \beta+\beta k z\right) \mathrm{e}^{-k z} J_{0}(k r),
\end{array}\right.
$$


where $\alpha, \beta$ and $k$ are arbitrary constants. Therefore, a more general solution is based on integrals having the form of Hankel transforms

$$
\left\{\begin{array}{l}
u_{r}(r, z)=\int_{0}^{\infty}\left(\alpha(k)-\frac{\lambda+2 \mu}{\lambda+\mu} \beta(k)+\beta(k) k z\right) \mathrm{e}^{-k z} J_{1}(k r) k d k \\
u_{z}(r, z)=\int_{0}^{\infty}\left(\alpha(k)+\frac{\mu}{\lambda+\mu} \beta(k)+\beta(k) k z\right) \mathrm{e}^{-k z} J_{0}(k r) k d k
\end{array},\right.
$$

where $\alpha(k)$ and $\beta(k)$ are now unknown functions. We can compute the relevant stress components

$$
\Theta_{r z}(r, z)=2 \mu \int_{0}^{\infty}(\beta(k)-\alpha(k)-\beta(k) k z) \mathrm{e}^{-k z} J_{1}(k r) k^{2} d k
$$

and

$$
\Theta_{z z}(r, z)=-2 \mu \int_{0}^{\infty}(\alpha(k)+\beta(k) k z) \mathrm{e}^{-k z} J_{0}(k r) k^{2} d k .
$$

The boundary condition $\Theta_{r z}(r, z=0)=0$ is satisfied by taking $\alpha=\beta$. A second boundary condition is

$$
\Theta_{z z}(r, z=0)=I(r),
$$

where $I(r)$ is, as seen above, the normalized intensity of the readout beam. Therefore, we have

$$
-2 \mu \int_{0}^{\infty} \alpha(k) J_{0}(k r) k^{2} d k=I(r) .
$$

Inverting the Hankel transform, we find

$$
k \alpha(k)=-\frac{1}{2 \mu} \tilde{I}(k)
$$

where $\tilde{I}(k)$ is the Hankel transform of the intensity

$$
\tilde{I}(k)=\int_{0}^{\infty} I(r) J_{0}(k r) r d r .
$$

The surface displacement is now, with $\alpha=\beta$,

$$
\begin{aligned}
u_{z}(r, z=0) & =\frac{\lambda+2 \mu}{\lambda+\mu} \int_{0}^{\infty} \alpha(k) J_{0}(k r) k d k \\
& =-\frac{\lambda+2 \mu}{2 \mu(\lambda+\mu)} \int_{0}^{\infty} \tilde{I}(k) J_{0}(k r) d k \\
& =-\frac{2\left(1-\sigma^{2}\right)}{Y} \int_{0}^{\infty} \tilde{I}(k) J_{0}(k r) d k,
\end{aligned}
$$

where we have switched from Lamé coefficients to the Poisson ratio $\sigma$ and Young's modulus $Y$. The strain energy $U$ is given by

$$
U=-\frac{1}{2} \int_{0}^{2 \pi} d \phi \int_{0}^{\infty} r d r I(r) u_{z}(r, 0)
$$

so that we get the general formula

$$
U=2 \pi \frac{1-\sigma^{2}}{Y} \varpi_{0},
$$

where

$$
\varpi_{0} \equiv \int_{0}^{\infty} \tilde{I}(k)^{2} d k
$$


Table 15: Some numerical values of $g_{0, n, m}$

\begin{tabular}{ll|cccccc}
\hline & $\mathrm{m}$ & 0 & 1 & 2 & 3 & 4 & 5 \\
$\mathrm{n}$ & & & & & & \\
\hline 0 & 1 & .60 & .46 & .39 & .34 & .31 \\
1 & & .69 & .50 & .41 & .36 & .32 & .29 \\
2 & & .57 & .44 & .37 & .33 & .30 & .28 \\
3 & & .50 & .40 & .35 & .31 & .29 & .27 \\
4 & .46 & .37 & .33 & .30 & .27 & .26 \\
5 & .43 & .35 & .31 & .28 & .26 & .25 \\
\hline
\end{tabular}

It is possible to get explicit results for Laguerre-Gauss and ideally flat beams. The Bessel transforms of the intensity profiles have already been calculated in Section 3.1.3. We have, for an $\mathrm{LG}_{n, m}$ beam of width $w$,

$$
\tilde{I}_{n, m}(k)=\frac{1}{2 \pi} \mathrm{e}^{-y} L_{m}(y) L_{n+m}(y), \quad\left(y \equiv \frac{k^{2} w^{2}}{8}\right)
$$

and for an ideally flat beam of radius $b$

$$
\tilde{I}_{\text {flat }}(k)=\frac{J_{1}(k b)}{\pi k b} .
$$

And now

$$
\varpi_{0, n, m}=\int_{0}^{\infty} \tilde{I}_{n, m}(k)^{2} d k=\frac{1}{4 \pi^{3 / 2} w} g_{0, n, m},
$$

where $g_{0, n, m}$ are numerical factors. The final result is

$$
U_{n, m}=\frac{1-\sigma^{2}}{2 \sqrt{\pi} Y w} g_{0, n, m} .
$$

The result for $n=0, m=0$ is first given in [5]. Table (15) gives an idea of the numerical values of $g_{0, n, m}$.

For larger values of $n$ and $m$, an increasingly good approximation is the asymptotic value

$$
g_{0, n, m} \sim(2 m+n+1)^{-1 / 2} .
$$

For an ideally flat mode, we get

$$
\varpi_{0, F}=\int_{0}^{\infty} \tilde{I}_{\text {flat }}(k)^{2} d k=\frac{4}{3 \pi^{3} b}
$$

so that

$$
U_{\text {flat }}=\frac{8\left(1-\sigma^{2}\right)}{3 \pi^{2} b Y} .
$$

These results are useful in order to compare with a more accurate calculation taking into account the finite dimensions of mirrors. For instance, with (Ex1) with a mirror (Virgo size) having a $35 \mathrm{~cm}$ diameter and an $\mathrm{LG}_{0,0}$ mode of width $2 \mathrm{~cm}$ (Virgo input mirror), we get

$$
\begin{gathered}
\varpi_{0}=2.245 \mathrm{~m}^{-1} \\
U_{0,0}=1.88 \times 10^{-10} \mathrm{~J} \mathrm{~N}^{-2}
\end{gathered}
$$


giving a noise linear spectral density of equivalent displacement:

$$
S_{x}^{1 / 2}(f)=9.95 \times 10^{-19}\left[\frac{1 \mathrm{~Hz}}{f}\right]^{1 / 2} \mathrm{mHz}^{-1 / 2} .
$$

Then (Ex2) for a flat beam of radius $9.1 \mathrm{~cm}$ gives

$$
\begin{gathered}
\varpi_{0}=0.473 \quad(\text { mesa }: 0.427) \mathrm{m}^{-1} \\
U_{\text {flat }}=3.95 \times 10^{-11} \mathrm{~J} \mathrm{~N}^{-2} \\
S_{x}^{1 / 2}(f)=4.56 \times 10^{-19}\left[\frac{1 \mathrm{~Hz}}{f}\right]^{1 / 2} \mathrm{mHz}^{-1 / 2} .
\end{gathered}
$$

For the mesa beam, we have

$$
S_{x}^{1 / 2}(f)=4.34 \times 10^{-19}\left[\frac{1 \mathrm{~Hz}}{f}\right]^{1 / 2} \mathrm{mHz}^{-1 / 2}
$$

and for an $\mathrm{LG}_{5,5}$ beam of width $3.5 \mathrm{~cm}(\mathrm{Ex} 3)$, this is

$$
\begin{gathered}
\varpi_{0}=0.321 \mathrm{~m}^{-1} \\
U_{55}=2.68 \times 10^{-11} \mathrm{~J} \mathrm{~N}^{-2} \\
S_{x}^{1 / 2}(f)=3.76 \times 10^{-19}\left[\frac{1 \mathrm{~Hz}}{f}\right]^{1 / 2} \mathrm{mHz}^{-1 / 2} .
\end{gathered}
$$

We will see in Section 8.4 that the first estimate is good (sharp spot on the center, far from the edge), whereas the last two (widely spread light power) are far from reality. This is why the infinite mirror approximation must be used with care.

\subsection{Infinite mirrors, noise in coating}

The dielectric coatings required to transform a polished blank of silica into a mirror are deposited by successive layers and form a region at the reflecting surface whose mechanical parameters are different from the bulk material. There is also a large difference for the loss angle $\Phi_{\text {coating }}$ compared to the substrate's. We shall treat the coating as a layer of thickness $\delta_{C}$, having a specific Young's modulus $Y_{C}$ and Poisson ratio $\sigma_{C}$, and corresponding Lamé coefficients $\lambda_{C}, \mu_{C}$. The coating is assumed to be located in the region $\left[-\delta_{C} \leq z \leq 0\right]$, so that the interface substrate/coating is located at $z=0$. For $z>0$, we have the expression (8.12) of the displacement vector (with $(\alpha, \beta)$ possibly different from the preceding solution (8.18) at the end). The following solution of the Navier-Cauchy equations for the displacement vector in the coating is easily found

$$
\begin{aligned}
& u_{r}(r, z)=\int_{0}^{\infty} A_{C}(k, z) J_{1}(k r) k d k \\
& u_{r}(r, z)=\int_{0}^{\infty} B_{C}(k, z) J_{0}(k r) k d k .
\end{aligned}
$$


But in the present case, the functions $A_{C}$ and $B_{C}$ are more complicated, taking into account two finite boundaries

$$
\begin{aligned}
A_{C}(k, z)= & \frac{\lambda_{C}+\mu_{C}}{2\left(\lambda_{C}+2 \mu_{C}\right)} k z\left[\gamma_{1} \cosh (k z)+\gamma_{2} \sinh (k z)\right]+\gamma_{3} \sinh (k z)+\gamma_{4} \cosh (k z) \\
B_{C}(k, z)= & \left(\frac{\lambda_{C}+3 \mu_{C}}{2\left(\lambda_{C}+2 \mu_{C}\right)} \gamma_{1}-\gamma_{3}\right) \cosh (k z)+\left(\frac{\lambda_{C}+3 \mu_{C}}{2\left(\lambda_{C}+2 \mu_{C}\right)} \gamma_{2}-\gamma_{4}\right) \sinh (k z) \\
& -\frac{\lambda_{C}+\mu_{C}}{2\left(\lambda_{C}+2 \mu_{C}\right)} k z\left[\gamma_{1} \sinh (k z)+\gamma_{2} \cosh (k z)\right] .
\end{aligned}
$$

The four arbitrary constant $\gamma_{1,2,3,4}$ can be related to $\alpha$ and $\beta$ of the preceding solution (8.12) by requiring continuity of the displacements and of the pressure components at the interface $z=0$. Then the boundary conditions are

$$
\left\{\begin{array}{l}
\Theta_{z z}\left(r,-\delta_{C}\right)=-I(r) \\
\Theta_{r z}\left(r,-\delta_{C}\right)=0
\end{array}\right.
$$

which allow one to compute $(\alpha, \beta)$ and find the complete solution. The exact solution is complicated and of little interest because we are in a case where $\delta_{C}$ (tens of microns) is small compared to the $w$ parameter of the beam (a few $\mathrm{cm}$ ). A solution at first order in $k \delta_{c}$ is therefore sufficient. We first find the energy stored in the bulk. It would be difficult to use the same method as in the preceding case (a surface integral). Instead we use the definition of the energy density

$$
w(r, z)=\frac{1}{2}\left[\lambda E^{2}(r, z)+2 \mu\left(E_{r r}^{2}(r, z)+E_{\phi \phi}^{2}(r, z)+2 E_{r z}^{2}(r, z)\right)\right] .
$$

We rewrite using the form

$$
\begin{aligned}
w(r, z)= & \frac{Y}{2(1+\sigma)(1-2 \sigma)}\left\{\sigma E(r, z)^{2}+(1-2 \sigma)\left[\left(E_{r r}(r, z)+E_{\phi \phi}(r, z)\right)^{2}\right.\right. \\
& \left.\left.-2 E_{r r}(r, z) E_{\phi \phi}(r, z)+E_{z z}(r, z)^{2}\right]\right\}
\end{aligned}
$$

and we integrate over the volume $[0 \leq \phi \leq 2 \pi] \times[0 \leq r \leq \infty] \times[0 \leq z \leq \infty]$. It is easy to see that the crossed term $E_{r r} E_{\phi \phi}$ does not contribute in an $r$ integral, being the derivative of a function null at $r=0$ and $r=\infty$. By using the closure relation

$$
\int_{0}^{\infty} J_{\nu}(k r) J_{\nu}\left(k^{\prime} r\right) r d r=\frac{\delta\left(k-k^{\prime}\right)}{k}
$$

one finally obtains

$$
U=2 \pi \frac{1-\sigma^{2}}{Y} \varpi_{0}+2 \pi \delta_{C} \frac{(1+\sigma)(1-2 \sigma)}{Y\left(1-\sigma_{C}\right)}\left[1-2 \sigma+\sigma_{C} \frac{Y}{Y_{C}} \frac{1+\sigma_{C}}{1+\sigma}\right] \varpi_{1}+\prime\left(\delta_{C}^{2}\right)
$$

with the notation

$$
\varpi_{1}=\int_{0}^{\infty} \tilde{I}^{2}(k) k d k .
$$

Note that, due to the Plancherel theorem (or to the closure relation in the direct space), this is also

$$
\varpi_{1}=\int_{0}^{\infty} I^{2}(r) r d r
$$


Table 16: Some numerical values of $g_{1, n, m}$

\begin{tabular}{cc|cccccc}
\hline & $\mathrm{m}$ & 0 & 1 & 2 & 3 & 4 & 5 \\
$\mathrm{n}$ & & & & & & \\
\hline 0 & & 1 & .50 & .34 & .27 & .22 & .19 \\
1 & & .50 & .31 & .23 & .19 & .16 & .14 \\
2 & & .38 & .25 & .19 & .16 & .14 & .12 \\
3 & & .31 & .21 & .17 & .14 & .12 & .11 \\
4 & .27 & .19 & .15 & .13 & .11 & .10 \\
5 & & .25 & .17 & .14 & .12 & .11 & .10 \\
\hline
\end{tabular}

Now the energy stored in the coating can be computed in the same way, by integrating the energy density

$$
\begin{aligned}
w_{C}(r, z)= & \frac{Y_{C}}{2\left(1+\sigma_{C}\right)\left(1-2 \sigma_{C}\right)}\left\{\sigma_{C} E(r, z)^{2}+\left(1-2 \sigma_{C}\right)\left[\left(E_{r r}(r, z)+E_{\phi \phi}(r, z)\right)^{2}\right.\right. \\
& \left.\left.-2 E_{r r}(r, z) E_{\phi \phi}(r, z)+E_{z z}(r, z)^{2}\right]\right\}
\end{aligned}
$$

within the volume $[0 \leq \phi \leq 2 \pi] \times[0 \leq r \leq \infty] \times\left[-\delta_{C} \leq z \leq 0\right]$. But at first order in $\delta_{C}$, it is sufficient to take

$$
U_{\text {coating }}=2 \pi \delta_{C} \int_{0}^{\infty} w_{C}(r, 0) r d r
$$

and we find

$$
U_{\text {coating }}=2 \pi \delta_{C} \frac{(1+\sigma)(1-2 \sigma)}{Y} \Omega_{1} \varpi_{1},
$$

where

$$
\Omega_{1}=\frac{1-2 \sigma_{C}}{2\left(1-\sigma_{C}\right)}\left[\frac{\left(1+\sigma_{C}\right) Y}{(1+\sigma)(1-2 \sigma) Y_{C}}+\frac{(1+\sigma) Y_{C}}{\left(1+\sigma_{C}\right) Y}\right]
$$

( $\Omega$ takes the value 1 when $Y=Y_{C}$ and $\sigma=\sigma_{C}$ ).

\subsubsection{Coating Brownian thermal noise: LG modes}

In the case of an $\mathrm{LG}_{n, m}$ mode, we get

$$
\varpi_{1, n, m}=\frac{1}{2 \pi^{2} w^{2}} g_{1, n, m},
$$

where $g_{1, n, m}$ is a numerical factor. Table 16 gives some values of $g_{1, n, m}$.

The strain energy stored in the coating is

$$
U_{n, m, \text { coating }}=\delta_{C} \frac{(1+\sigma)(1-2 \sigma)}{\pi Y w^{2}} \Omega_{1} g_{1, n, m} .
$$

Thus, the ratio between the main energy in the substrate and that in the preceding coating is

$$
\varrho \equiv \frac{U_{n, m, \text { coating }}}{U_{n, m, \text { substrate }}}=\frac{2}{\sqrt{\pi}} \frac{\delta_{C}}{w} \frac{1-2 \sigma}{1-\sigma} \Omega_{1} \frac{g_{1, n, m}}{g_{0, n, m}} .
$$

A similar result has been also reported by [32] and [18] in the case of $(n=0, m=0)$ at the limit $\Omega_{1}=1$. For reasonable parameters, $\Omega_{1}$ is not so different from one. In the case of the Virgo cavity 
input mirrors (Ex1), assuming a stack $25 \mu \mathrm{m}$ thick, and elastic parameters $\left(Y_{C} \sim 1.4 \times 10^{11} \mathrm{~N} \mathrm{~m}^{-2}\right.$, $\left.\sigma_{C} \sim 0.23\right), \Omega_{1} \sim 0.93$ and we get

$$
\varrho \sim 10^{-3}
$$

and, for an $\mathrm{LG}_{5,5}$ mode of width $w=3.5 \mathrm{~cm}(\mathrm{Ex} 3)$,

$$
\varrho \sim 2.4 \times 10^{-4}
$$

\subsubsection{Coating Brownian thermal noise: Flat modes}

The Fourier transform of the flat mode pressure was found in Section 3.1.3:

$$
\tilde{I}_{\text {flat }}(k)=\frac{J_{1}(k b)}{\pi k b} .
$$

Thus, we have

$$
\varpi_{1, F}=\frac{1}{\pi^{2} b^{2}} \int_{0}^{\infty} \frac{J_{1}(x)^{2}}{x} d x=\frac{1}{2 \pi^{2} b^{2}}
$$

and the coating strain energy is

$$
U_{F, \text { coating }}=\frac{(1+\sigma)(1-2 \sigma)}{Y} \Omega_{1} \frac{\delta_{C}}{\pi b^{2}}
$$

with the ratio $\varrho$ now

$$
\varrho=\frac{U_{F, \text { coating }}}{U_{\text {flat }}}=\frac{3 \pi}{8} \frac{\delta_{C}}{b} \frac{1-2 \sigma}{1-\sigma} .
$$

For the flat mode of (Ex2), we find

$$
\varrho \sim 2.6 \times 10^{-4}
$$

Here are some numerical values for comparison. For the $\mathrm{LG}_{5,5}$ mode, we have $\varpi_{1,5,5} \sim 4.14 \mathrm{~m}^{-2}$, For the flat mode $(b=9.1 \mathrm{~cm})$, we have $\varpi_{1 \text {,flat }} \sim 6.12 \mathrm{~m}^{-2}$ (to be discarded, as the sharp edge effect becomes spurious, see the next value), $\varpi_{1 \text {, mesa }} \sim 4.52 \mathrm{~m}^{-2}$ (numerical integration), and for the Gauss-Bessel mode of Figure 5 , this is $\varpi_{1, \mathrm{~GB}} \sim 3.56 \mathrm{~m}^{-2}$. A value of $2.34 \mathrm{~m}^{-2}$ is reported by [4] after an optimization process involving an expansion on LG modes. It is probably possible to have a not too different result by a fine tuning of the conical mode's parameters.

\subsection{Finite mirrors}

The treatment of a mirror as an infinite medium can be accepted when the readout beam is a sharp one, of width much smaller than the mirror's dimensions. But it becomes highly questionable when the spatial extension of the mode is comparable to those dimensions, which is precisely the case when high-order LG modes or mesa beams are considered. This is why an analytic method for computing $U$ in the case of finite size mirrors has been developed in BHV, then amended in [28] for one boundary condition. We change slightly the coordinate system with respect to the preceding thermal studies. The mirror is assumed to fill the cylindrical volume defined by $r \in[0, a], z \in[0, h]$. The reflecting face (intracavity) is assumed to be at $z=0$. As in the thermal studies, we take a displacement vector under the form of an FB series

$$
\left\{\begin{array}{l}
u_{r}(r, z)=\sum_{s} A_{s}(z) J_{1}\left(k_{s} r\right) \\
u_{\phi}(r, z)=0 \\
u_{z}(r, z)=\sum_{s} B_{s}(r, z) J_{0}\left(k_{s} r\right)
\end{array}\right.
$$

where $A_{s}$ and $B_{s}$ are unknown functions of $z$, and $k_{s}$ are constants to be determined. 


\subsubsection{Equilibrium equations}

The Navier-Cauchy equations read

$$
\left\{\begin{array}{l}
\mu\left(\partial_{z}^{2}-k_{s}^{2}\right) A_{s}-(\lambda+\mu) k_{s}\left(\partial_{z} B_{s}+k_{s} A_{s}\right) \\
\mu\left(\partial_{z}^{2}-k_{s}^{2}\right) B_{s}+(\lambda+\mu) \partial_{z}\left(\partial_{z} B_{s}+k_{s} A_{s}\right)
\end{array},\right.
$$

which yields

$$
\left(\partial_{z}^{2}-k_{s}^{2}\right)\left(\partial_{z} A_{s}+k_{s} B_{s}\right)=0
$$

The general solution of which is

$$
\partial_{z} A_{s}+k_{s} B_{s}=C_{s} \mathrm{e}^{-k_{s} z}+D_{s} \mathrm{e}^{k_{s} z} .
$$

This allows one to compute $B_{s}$ in terms of $A_{s}$ and to substitute it in Equation (8.67), so that one gets

$$
\left(\partial_{z}^{2}-k_{s}^{2}\right) A_{s}=-\frac{\lambda+\mu}{\lambda+2 \mu} k_{s}^{2}\left(C_{s} \mathrm{e}^{-k_{s} z}-D_{s} \mathrm{e}^{k_{s} z}\right) .
$$

The solution of which is

$$
A_{s}(z)=M_{s} \mathrm{e}^{-k_{s} z}+N_{s} \mathrm{e}^{k_{s} z}+\frac{\lambda+\mu}{2(\lambda+2 \mu)} k_{s} z\left(C_{s} \mathrm{e}^{-k_{s} z}+D_{s} \mathrm{e}^{k_{s} z}\right)
$$

where $M_{s}$ and $N_{s}$ are two more arbitrary constants. Now $B_{s}$ is determined by

$$
\begin{aligned}
B_{s}(z)= & \left(\frac{\lambda+3 \mu}{2(\lambda+2 \mu)} C_{s}+M_{s}\right) \mathrm{e}^{-k_{s} z}+\left(\frac{\lambda+3 \mu}{2(\lambda+2 \mu)} D_{s}-N_{s}\right) \mathrm{e}^{k_{s} z}+ \\
& \frac{\lambda+\mu}{2(\lambda+2 \mu)} k_{s} z\left(C_{s} \mathrm{e}^{-k_{s} z}-D_{s} \mathrm{e}^{k_{s} z}\right)
\end{aligned}
$$

\subsubsection{Boundary conditions}

The boundary conditions we assume are

- No shear on the cylindrical edge, i.e.,

$$
\Theta_{r z}(a, z)=0 \text {. }
$$

This can be satisfied by requiring $\zeta_{s} \equiv k_{s} a$ to be a strictly positive zero of $J_{1}(\zeta)$. The family of all such zeroes defines a family of functions $\left\{J_{0}\left(\zeta_{s} r / a\right), s \in \mathbb{N}^{*}\right\}$ complete and orthogonal on $[0, a]$, on which any function of $r$ may be expanded as a FB series. Note that this family is different from the families encountered in thermal studies. In particular, the orthogonality relation is simpler:

$$
\int_{0}^{a} J_{0}\left(k_{s} r\right) J_{0}\left(k_{s^{\prime}} r\right) r d r=\frac{a^{2}}{2} J_{0}^{2}\left(\zeta_{s}\right) \delta_{s, s^{\prime}}
$$

- No shear on the two circular faces, i.e.,

$$
\Theta_{r z}(a, 0)=\Theta_{r z}(a, h)=0 .
$$

- The given pressure on the reflecting face:

$$
\Theta_{z z}(r, 0)=-p(r),
$$

where $p(r)$ is a pressure distribution normalized to an integrated force of $1 \mathrm{~N}$, identical to the normalized optical intensity function $I(r)$. 
- No pressure on the rear face:

$$
\Theta_{z z}(r, h)=0
$$

- No radial stress on the edge:

$$
\Theta_{r r}(a, z)=0 .
$$

The pressure distribution can, as usual, be expanded on the complete orthogonal family $J_{0}\left(k_{s} z\right)$ :

$$
p(r)=I(r)=\frac{1}{\pi a^{2}} \sum_{s} p_{s} J_{0}\left(k_{s} r\right) .
$$

Owing to the norm of the functions $J_{0}\left(k_{s} r\right)$, the FB coefficients $p_{s}$ are now defined by

$$
p_{s}=\frac{2 \pi}{J_{0}^{2}\left(\zeta_{s}\right)} \int_{0}^{a} I(r) J_{0}\left(k_{s} r\right) r d r
$$

We have already encountered this kind of integral. The general result for an $\mathrm{LG}_{n, m}$ mode is

$$
p_{m, s}^{(n)}=\frac{1}{J_{0}^{2}\left(\zeta_{s}\right)} \exp \left(-y_{s}\right) L_{m}(y) L_{m+n}(y) \quad\left(y_{s} \equiv k_{s}^{2} w^{2} / 8\right)
$$

and for a flat mode

$$
p_{\text {flat }, s}=\frac{2 a J_{1}\left(\zeta_{s} b / a\right)}{b J_{0}^{2}\left(\zeta_{s}\right)}
$$

For a mesa mode, numerical integration is necessary. The $\Theta_{r z}$ and $\Theta_{z z}$ components of the stress tensor are obtained as FB series:

$$
\begin{aligned}
& \Theta_{r z}(r, z)=\sum_{s} \Theta_{s, r z}(z) J_{1}\left(k_{s} r\right) \\
& \Theta_{z z}(r, z)=\sum_{s} \Theta_{s, z z}(z) J_{0}\left(k_{s} r\right),
\end{aligned}
$$

making clear that the first boundary condition is satisfied. In more detail, we have

$$
\begin{gathered}
\Theta_{s, r z}(z) / k_{s} \mu=\left(2 N_{s}-\frac{\mu}{\lambda+2 \mu} D_{s}\right) \mathrm{e}^{k_{s} z}-\left(2 M_{s}-\frac{\mu}{\lambda+2 \mu} C_{s}\right) \mathrm{e}^{-k_{s} z} \\
+\frac{\lambda+\mu}{\lambda+2 \mu} k_{s} z\left(C_{s} \mathrm{e}^{-k_{s} z}-D_{s} \mathrm{e}^{k_{s} z}\right) \\
\Theta_{s, z z}(z) / k_{s} \mu=\left(D_{s}-2 N_{s}\right) \mathrm{e}^{k_{s} z}-\left(C_{s}+2 M_{s}\right) \mathrm{e}^{-k_{s} z}-\frac{\lambda+\mu}{\lambda+2 \mu} k_{s} z\left(C_{s} \mathrm{e}^{-k_{s} z}+D_{s} \mathrm{e}^{k_{s} z}\right) .
\end{gathered}
$$

The boundary conditions on the faces provide four equations allowing one to determine the constants $C_{s}, D_{s}, M_{s}$, and $N_{s}$. We have

$$
\begin{gathered}
C_{s}=\frac{1}{\pi a^{2}} \frac{4 p_{s}\left(1-\sigma^{2}\right)}{k_{s} Y} \frac{1-q_{s}+2 q_{s} x_{s}}{\left(1-q_{s}\right)^{2}-4 q_{s} x_{s}^{2}} \\
D_{s}=\frac{1}{\pi a^{2}} \frac{4 p_{s}\left(1-\sigma^{2}\right)}{k_{s} Y} \frac{q_{s}\left(1-q_{s}+2 x_{s}\right)}{\left(1-q_{s}\right)^{2}-4 q_{s} x_{s}^{2}} \\
M_{s}=-\frac{1}{\pi a^{2}} \frac{p_{s}(1+\sigma)}{k_{s} Y} \frac{2 q_{s} x_{s}^{2}+(1-2 \sigma)\left(1-q_{s}+2 q_{s} x_{s}\right)}{\left(1-q_{s}\right)^{2}-4 q_{s} x_{s}^{2}}
\end{gathered}
$$




$$
N_{s}=-\frac{1}{\pi a^{2}} \frac{p_{s} q_{s}(1+\sigma)}{k_{s} Y} \frac{2 x_{s}^{2}-(1-2 \sigma)\left(1-q_{s}+2 x_{s}\right)}{\left(1-q_{s}\right)^{2}-4 q_{s} x_{s}^{2}},
$$

with the notation $x_{s} \equiv k_{s} h$ and $q_{s} \equiv \exp \left(-2 x_{s}\right)$. This was found by [5]. At this point, [28] pointed out that the component of spatial frequency zero of the pressure was not taken into account (recall that the $\zeta_{s}$ are the nonzero solutions of $\left.J_{1}(\zeta)=0\right)$. The preceding displacement vector has a zero average on the strained face. One must now consider the resulting force acting on the body under the uniform pressure

$$
p_{0}=\frac{1}{\pi a^{2}}
$$

producing a force of $1 \mathrm{~N}$ after integration on the disk. But this force produces an acceleration, which should be added to the Navier-Cauchy equations (8.67) (recall that the mirrors of GW interferometers are practically free in the longitudinal degree of freedom in the detection band). This effect can be taken into account by an extra displacement of the form

$$
\left\{\begin{array}{l}
\delta_{1} u_{r}(r, z)=p_{0} \frac{\sigma}{Y} r(1-z / h) \\
\delta_{1} u_{z}(r, z)=p_{0}\left[\frac{\sigma}{Y} \frac{r^{2}}{2 h}-\frac{1}{Y}\left(z-z^{2} / 2 h\right)\right] .
\end{array}\right.
$$

This extra displacement contributes only axial stress

$$
\delta_{1} \Theta_{z z}=-p_{0}(1-z / h)
$$

all other extra stress components being null. The equilibrium equations remain satisfied for

$$
\partial_{z} \delta_{1} \Theta_{z z}=p_{0} / h=\rho \times \frac{1 \mathrm{~N}}{\rho h \pi a^{2}}=\rho \times \frac{1 \mathrm{~N}}{\text { Mass }}=\rho \ddot{z}
$$

as remarked by [28]. Now, the sum of the displacement (8.66) and of the extra displacement (8.92) satisfies all boundary conditions except the vanishing of the radial stress on the edge. We have for the FB component of the radial stress

$$
\Theta_{s, r r}(z)=\lambda\left(k_{s} A_{s}(z)+\partial_{z} B_{s}(z)\right) J_{0}\left(\zeta_{s}\right)+2 \mu k_{s} A_{s} J_{1}^{\prime}\left(\zeta_{s}\right)
$$

But, due to the fact that $J_{1}^{\prime}\left(\zeta_{s}\right)=J_{0}\left(\zeta_{s}\right)$, and after substituting the explicit expressions of $A_{s}$ and $B_{s}$, we get

$$
\begin{aligned}
\Theta_{s, r r}(z)= & -p_{0} \frac{p_{s} J_{0}\left(\zeta_{s}\right)}{\left(1-q_{s}\right)^{2}-4 q_{s} x_{s}^{2}}\left[\left(1-q_{s}+2 q_{s} x_{s}\left(1+x_{s}\right)\right) \mathrm{e}^{-k_{s} z}-q_{s}\left(1-q_{s}+2 x_{s}\left(1-x_{s}\right)\right) \mathrm{e}^{k_{s} z}\right. \\
& \left.-k_{s} z q_{s}\left(1-q_{s}+2 x_{s}\right) \mathrm{e}^{k_{s} z}-k_{s} z\left(1-q_{s}+2 q_{s} x_{s}\right) \mathrm{e}^{-k_{s} z} .\right]
\end{aligned}
$$

It is numerically easy to check that this function of $z$ is not very different from linear. It has a vanishing average. Therefore, it is possible to find an approximate solution using the Saint-Venant principle once more. We add to our displacement one more extra displacement giving a linear edge stress compensating the preceding. The second extra displacement is of the form

$$
\left\{\begin{array}{l}
\delta_{2} u_{r}(r, z)=\frac{1-\sigma}{Y}\left(\omega_{0}+\omega_{1} z\right) r \\
\delta_{2} u_{z}(r, z)=-\frac{2 \sigma}{Y}\left(\omega_{0}+\omega_{1} z / 2\right) z-\frac{1-\sigma}{Y} \omega_{1} \frac{r^{2}}{2}
\end{array}\right.
$$

such that the equilibrium equations (8.67) are identically satisfied. It contributes only radial stress, thus leaving unchanged the boundary conditions, except for a radial contribution

$$
\delta_{2} \Theta_{r r}(z)=\omega_{0}+\omega_{1} z .
$$


As usual, we require a minimum residual stress on the edge, which amounts to having null resulting mean force and torque on the edge. If we define

$$
\begin{gathered}
I_{0}=\frac{1}{h} \int_{0}^{h} \Theta_{r r}(a, z) d z \\
I_{1}=\frac{1}{h^{2}} \int_{0}^{h} \Theta_{r r}(a, z) z d z
\end{gathered}
$$

then the values of $\omega_{0}$ and $\omega_{1}$ are

$$
\begin{gathered}
\omega_{0}=6 I_{1}-4 I_{0} \\
\omega_{1}=\left(6 I_{0}-12 I_{1}\right) / h .
\end{gathered}
$$

The explicit expression (8.96) allows one to compute $I_{0}$ and $I_{1}$. One finds

$$
\begin{gathered}
I_{0}=0 \\
I_{1}=p_{0} S
\end{gathered}
$$

with

$$
S=\frac{a^{2}}{h^{2}} \sum_{s} \frac{p_{s} J_{0}\left(\zeta_{s}\right)}{\zeta_{s}^{2}}
$$

and

$$
\omega_{0}=6 S p_{0}, \quad \omega_{1}=-12 S p_{0} / h .
$$

In summary, the total displacement is now

$$
\vec{u}_{\text {tot }}(r, z)=\vec{u}(r, z)+\Delta \vec{u}(r, z)
$$

with

$$
\left\{\begin{array}{l}
u_{r}(r, z)=\sum A_{s}(z) J_{1}\left(k_{s} r\right) \\
u_{z}(r, z)=\sum B_{s}(z) J_{0}\left(k_{s} r\right)
\end{array}\right.
$$

and

$$
\begin{aligned}
\Delta \vec{u}(r, z) & =\delta_{1} \vec{u}(r, z)+\delta_{2} \vec{u}(r, z) \\
& =\left\{\begin{array}{l}
p_{0} \frac{\sigma}{Y}(1-z / h)+\frac{1-\sigma}{Y}\left(\omega_{0}+\omega_{1} z\right) r \\
p_{0} \frac{\sigma}{2 Y} r^{2} / h-\frac{1}{Y}\left(z-z^{2} / 2 h\right)-\frac{2 \sigma}{Y}\left(\omega_{0} z+\omega_{1} z^{2} / 2\right)-\frac{1-\sigma}{2 Y} \omega_{1} r^{2}
\end{array}\right.
\end{aligned}
$$

\subsubsection{Strain energy}

The strain tensor is now of the form

$$
E_{i j}(r, z)=E_{0, i j}(r, z)+\Delta E_{i j}(z),
$$

where the component $E_{0, i j}$ is computed from $\vec{u}$, whereas the component $\Delta E_{i j}$ is computed from $\Delta \vec{u}$. Now the strain energy density $w(r, z)$ is defined by

$$
\begin{aligned}
w(r, z)= & \frac{1}{2} \frac{Y}{(1+\sigma)(1-2 \sigma)}\left[\sigma E(r, z)^{2}+(1-2 \sigma)\left(E_{r r}(r, z)^{2}\right.\right. \\
& \left.\left.+E_{\phi \phi}(r, z)^{2}+E_{z z}(r, z)^{2}+2 E_{r z}(r, z)^{2}\right)\right]
\end{aligned}
$$


$E(r, z) \equiv \sum_{i} E_{i i}(r, z)$ being the trace of the strain tensor. Thus, integrated strain energy $U$, i.e., our target, is

$$
U=\int_{0}^{2 \pi} d \phi \int_{0}^{h} d z \int_{0}^{a} r d r w(r, z)
$$

The squares of the strain tensor components obviously contain, in general, squares of the main strain, squares of the extra strains and cross products. However, in the $r$ integral, cross products vanish, so that the total internal energy is the sum of two contributions

$$
U=U_{0}+\Delta U
$$

These can be computed separately. We have

$$
U_{0}=\frac{1-\sigma^{2}}{\pi a Y} \sum_{s>0} \frac{J_{0}^{2}\left(\zeta_{s}\right) p_{s}^{2}}{\zeta_{s}} \frac{1-q_{s}^{2}+4 q_{s} x_{s}}{\left(1-q_{s}\right)^{2}-4 q_{s} x_{s}^{2}} .
$$

The dimension of $U$ is $\mathrm{J} \mathrm{N}^{-2}$. And for the second contribution, we have

$$
\Delta U=\frac{a^{2}}{6 \pi h^{3} Y}\left[\left(\frac{h}{a}\right)^{4}+12 \sigma \xi\left(\frac{h}{a}\right)^{2}+72(1-\sigma) \xi^{2}\right]
$$

with

$$
\xi \equiv \sum_{s>0} p_{s} J_{0}\left(\zeta_{s}\right) / \zeta_{s}^{2}
$$

For our three reference examples, using Virgo mirrors, we get, with a loss angle of $10^{-6}$,

- $\mathrm{LG}_{0,0}, w=2 \mathrm{~cm}$

$$
\begin{gathered}
U_{0}=1.81 \times 10^{-10} \mathrm{~J} \mathrm{~N}^{-2} \\
\Delta U=2.08 \times 10^{-11} \mathrm{~J} \mathrm{~N}^{-2} \\
U=2.02 \times 10^{-10} \mathrm{~J} \mathrm{~N}^{-2} \\
S_{x}^{1 / 2}(f)=1.03 \times 10^{-18}\left[\frac{1 \mathrm{~Hz}}{f}\right]^{1 / 2} \mathrm{mHz}^{-1 / 2}
\end{gathered}
$$

- flat mode, $b=9.1 \mathrm{~cm}$

$$
\begin{gathered}
U_{0}=2.08 \times 10^{-11} \mathrm{~J} \mathrm{~N}^{-2} \\
\Delta U=1.21 \times 10^{-11} \mathrm{~J} \mathrm{~N}^{-2} \\
U=3.28 \times 10^{-11} \mathrm{~J} \mathrm{~N}^{-2} \\
S_{x}^{1 / 2}(f)=4.16 \times 10^{-19}\left[\frac{1 \mathrm{~Hz}}{f}\right]^{1 / 2} \mathrm{mHz}^{-1 / 2}
\end{gathered}
$$


- mesa mode, $b_{f}=10.7 \mathrm{~cm}$

$$
\begin{gathered}
U_{0}=1.58 \times 10^{-11} \mathrm{~J} \mathrm{~N}^{-2} \\
\Delta U=1.04 \times 10^{-11} \mathrm{~J} \mathrm{~N}^{-2} \\
U=2.62 \times 10^{-11} \mathrm{~J} \mathrm{~N}^{-2} \\
S_{x}^{1 / 2}(f)=3.72 \times 10^{-19}\left[\frac{1 \mathrm{~Hz}}{f}\right]^{1 / 2} \mathrm{mHz}^{-1 / 2}
\end{gathered}
$$

- $\mathrm{LG}_{5,5}, w=3.5 \mathrm{~cm}$

$$
\begin{gathered}
U_{0}=4.24 \times 10^{-12} \mathrm{~J} \mathrm{~N}^{-2} \\
\Delta U=4.40 \times 10^{-12} \mathrm{~J} \mathrm{~N}^{-2} \\
U=8.64 \times 10^{-12} \mathrm{~J} \mathrm{~N}^{-2} \\
S_{x}^{1 / 2}(f)=2.13 \times 10^{-19}\left[\frac{1 \mathrm{~Hz}}{f}\right]^{1 / 2} \mathrm{mHz}^{-1 / 2}
\end{gathered}
$$

It is clear that for modes widely spread on the mirror surface, the Saint-Venant correction becomes important. Moreover, if we compare to the values found in the infinite mirror approximation, we see that the first example was underestimated by about $7 \%$, the flat mode by $17 \%$, and the third by a factor of 3 . We also see the discrepancy $(11 \%)$ between the flat estimation and the mesa beam. This leads us to be cautious with the foregoing estimations. Figure 58 summarizes the gain in thermal noise obtained with respect to the current situation on Virgo input mirrors for several beams having 1 ppm clipping losses.

\subsubsection{Explicit displacement and strain tensor}

In Section 9, we shall need the explicit expressions of the displacement vector and particularly of the trace of the strain tensor. We have, after the preceding calculations for the FB components of the main displacement,

$$
\begin{aligned}
A_{s}(z)= & \frac{p_{s}(1+\sigma)}{\pi a \zeta_{s} Y \Delta_{s}}\left\{-\left[(1-2 \sigma) Q_{s}+2 q_{s} x_{s}^{2}\right] \mathrm{e}^{-k_{s} z}+\left[(1-2 \sigma) R_{s}-2 q_{s} x_{s}^{2}\right] \mathrm{e}^{k_{s} z}\right. \\
& \left.+k_{s} z\left[Q_{s} \mathrm{e}^{-k_{s} z}+R_{s} \mathrm{e}^{k_{s} z}\right]\right\}
\end{aligned}
$$

with the notation

$$
\begin{aligned}
k_{s} & \equiv \zeta_{s} / a, \quad x_{s} \equiv k_{s} h, \quad q_{s} \equiv \exp \left(-2 x_{s}\right), \quad \Delta_{s} \equiv\left(1-q_{s}\right)^{2}-4 q_{s} x_{s}^{2}, \\
Q_{s} & \equiv 1-q_{s}+2 q_{s} x_{s}, \quad R_{s} \equiv q_{s}\left(1-q_{q}+2 x_{s}\right) .
\end{aligned}
$$

In the same way

$$
\begin{aligned}
B_{s}(z)= & \frac{p_{s}(1+\sigma)}{\pi a \zeta_{s} Y \Delta_{s}}\left\{\left[2(1-\sigma) Q_{s}-2 q_{s} x_{s}^{2}\right] \mathrm{e}^{-k_{s} z}+\left[2(1-\sigma) R_{s}+2 q_{s} x_{s}^{2}\right] \mathrm{e}^{k_{s} z}\right. \\
& \left.+k_{s} z\left[Q_{s} \mathrm{e}^{-k_{s} z}-R_{s} \mathrm{e}^{k_{s} z}\right]\right\}
\end{aligned}
$$




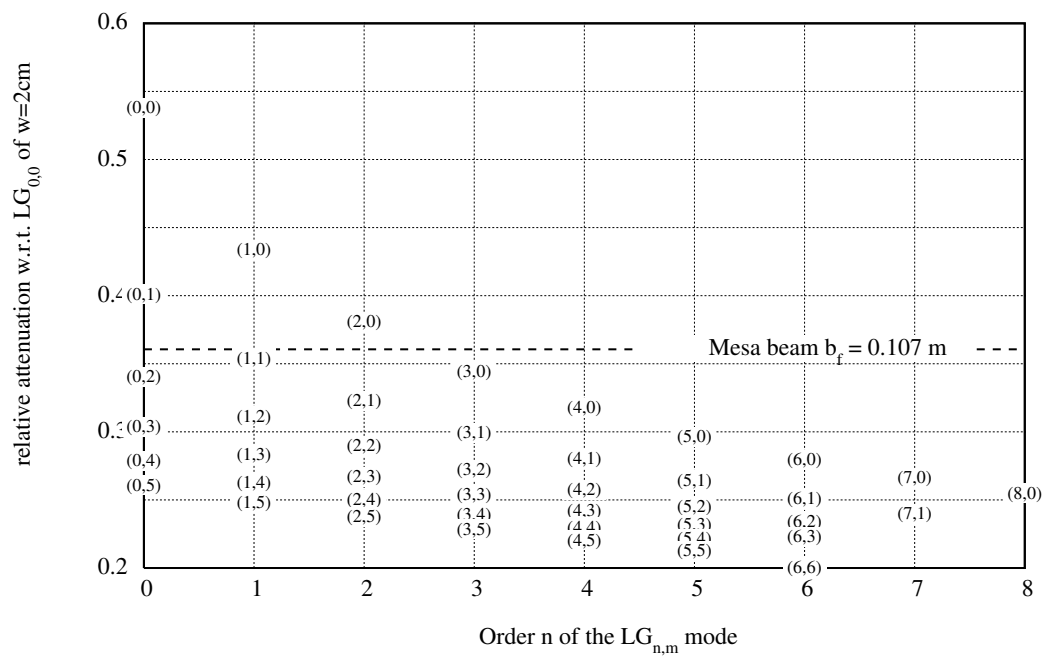

Figure 58: Gain in thermal noise $\mathrm{PSD}^{1 / 2}$ for LG modes having each a $w$ parameter tuned for $1 \mathrm{ppm}$ clipping losses, with respect to the Virgo input mirrors and beams

The $z$ derivative of $B_{s}(z)$ is needed as well,

$$
\begin{aligned}
\partial_{z} B_{s}(z)= & \frac{p_{s}(1+\sigma)}{\pi a^{2} Y \Delta_{s}}\left\{-\left[(1-2 \sigma) Q_{s}-2 q_{s} x_{s}^{2}\right] \mathrm{e}^{-k_{s} z}+\left[(1-2 \sigma) R_{s}+2 q_{s} x_{s}^{2}\right] \mathrm{e}^{k_{s} z}\right. \\
& \left.-k_{s} z\left[Q_{s} \mathrm{e}^{-k_{s} z}+R_{s} \mathrm{e}^{k_{s} z}\right]\right\}
\end{aligned}
$$

and the combination is still

$$
\frac{1}{2}\left[\partial_{z} A_{s}(z)-k_{s} B_{s}(z)\right]=\frac{(1+\sigma) p_{s}}{\pi a^{2} Y \Delta_{s}}\left\{\left(2 q_{s} x_{s}^{2}-k_{s} z Q_{s}\right) \mathrm{e}^{-k_{s} z}-\left(2 q_{s} x_{s}^{2}-k_{s} z R_{s}\right) \mathrm{e}^{k_{s} z}\right\} .
$$

For the extra displacement we have

$$
\begin{gathered}
\Delta u_{r}(r, z)=\frac{1}{\pi a^{2} Y}\left[c_{0}+c_{1} z\right] r \\
\Delta u_{z}(r, z)=\frac{1}{\pi a^{2} Y}\left[c_{2} r^{2}+c_{3} z+c_{4} z^{2}\right]
\end{gathered}
$$

with the notation

$$
\begin{aligned}
& c_{0}=\sigma+6(1-\sigma) S, \quad c_{1}=-[\sigma+12(1-\sigma) S] / h, \quad c_{2}=-\frac{1}{2} c_{1}, \\
& c_{3}=-(1+12 \sigma S), \quad c_{4}=(1+24 \sigma S) / 2 h
\end{aligned}
$$

This allows one to plot the (virtually) deformed solid (see Figure 59) in our three examples. We have amplified the displacement by a large factor, to give a better idea of the shape.

We find the FB components of the main strain tensor to be

$$
E_{r r}(r, z)=\sum_{s} k_{s} A_{s}(z) J_{1}^{\prime}\left(k_{s} r\right)
$$




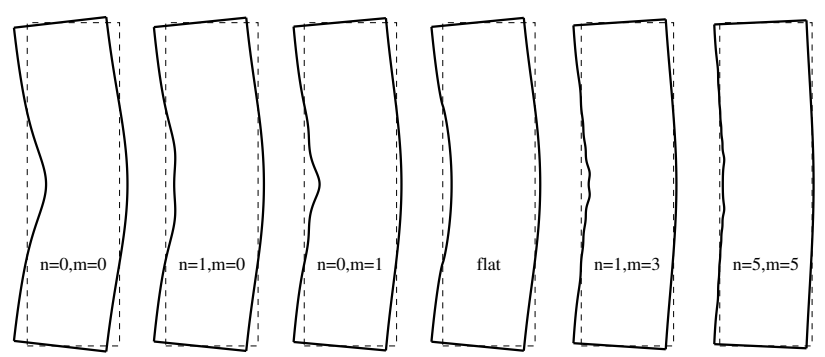

Figure 59: Virtually deformed mirror under beam pressure. (1 $\mathrm{N}$ integrated pressure) by modes having $1 \mathrm{ppm}$ clipping losses. For more clarity, the displacements have been amplified by a factor of $7 \times 10^{7}$.

$$
\begin{gathered}
E_{\phi \phi}(r, z)=\sum_{s} k_{s} A_{s}(z) \frac{J_{1}\left(k_{s} r\right)}{k_{s} r} \\
E_{z z}(r, z)=\sum_{s} \partial_{z} B_{s} J_{0}\left(k_{s} r\right) \\
E_{r z}(r, z)=\frac{1}{2} \sum_{s}\left(\partial_{z} A_{s}-k_{s} B_{s}\right) J_{1}\left(k_{s} r\right)
\end{gathered}
$$

This gives, in particular, the FB component of the trace of the strain tensor

$$
E(r, z)=\sum E_{s}(z) J_{0}\left(k_{s} r\right)
$$

with

$$
E_{s}(z)=-\frac{2(1+\sigma)(1-2 \sigma) p_{s}}{\pi a^{2} Y \Delta_{s}}\left[Q_{s} \mathrm{e}^{-k_{s} z}-R_{s} \mathrm{e}^{k_{s} z}\right]
$$

And for the extra contributions, we get

$$
\begin{gathered}
\Delta E_{r r}(z)=\frac{1}{\pi a^{2} Y}\left(c_{0}+c_{1} z\right) \\
\Delta E_{\phi \phi}(z)=\frac{1}{\pi a^{2} Y}\left(c_{0}+c_{1} z\right) \\
\Delta E_{z z}(z)=\frac{1}{\pi a^{2} Y}\left(c_{3}+2 c_{4} z\right) \\
\Delta E_{r z}=0 \\
\Delta E(z)=\frac{1}{\pi a^{2} Y}\left(2 c_{0}+c_{3}+2\left(c_{1}+c_{4}\right) z\right) \\
=-(1-2 \sigma) \frac{1}{\pi a^{2} Y}[1-12 S-(1-24 S) z / h]
\end{gathered}
$$

This allows us to map the energy density in the material (see Figures 60, 61, and 62). 


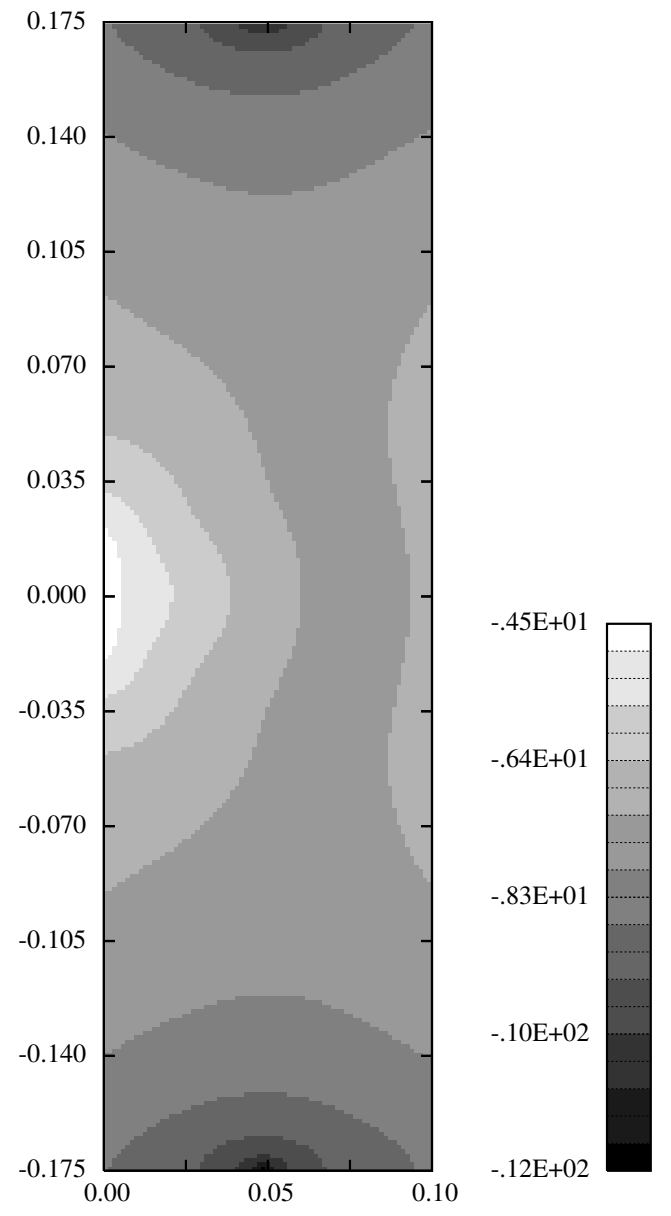

Figure 60: Distribution of strain energy in the mirror substrate $\left(\mathrm{LG}_{0,0} w=2 \mathrm{~cm}\right.$, logarithmic scale) 


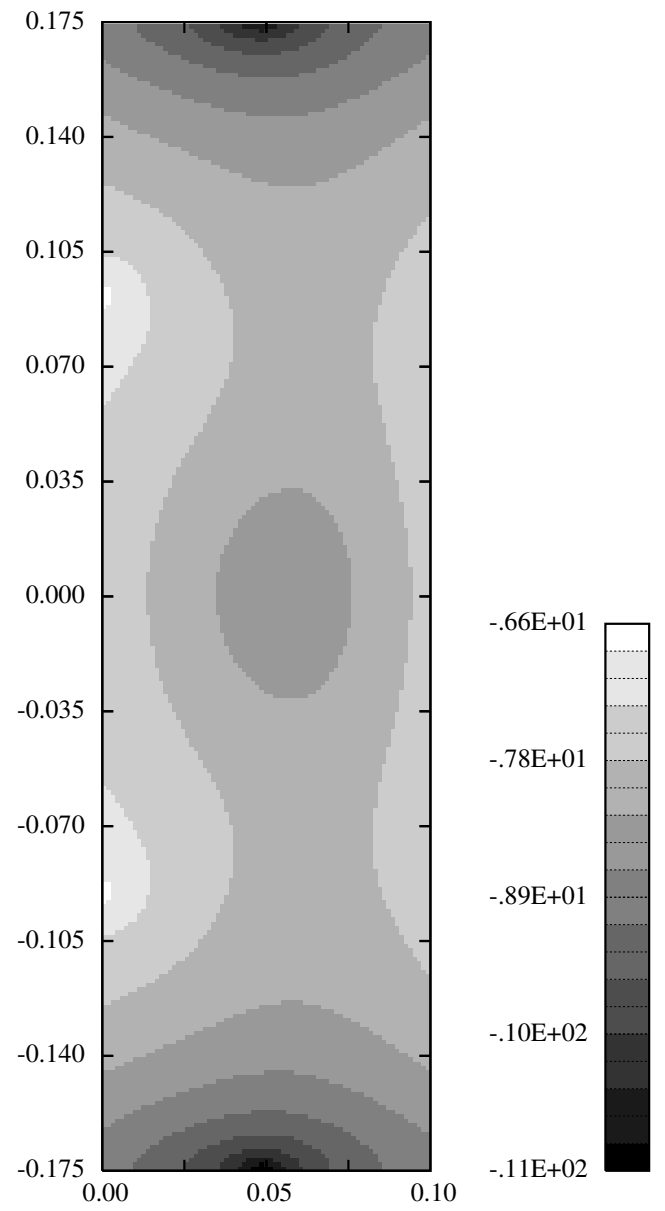

Figure 61: Distribution of strain energy in the mirror substrate (Flat mode $b=9.1 \mathrm{~cm}$, logarithmic scale) 


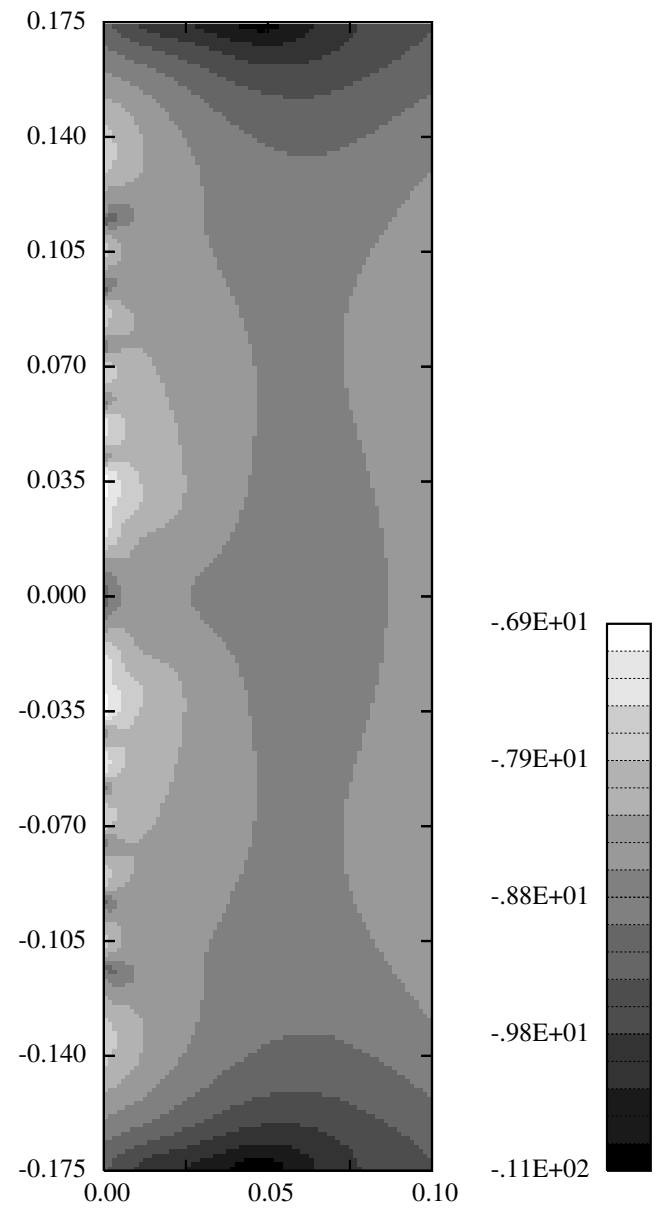

Figure 62: Distribution of strain energy in the mirror substrate $\left(\mathrm{LG}_{5,5} w=3.5 \mathrm{~cm}\right.$, logarithmic scale) 


\subsection{Coating Brownian thermal noise: finite mirrors}

We again consider the coating as a very thin, but finite, layer having a different loss angle. Thus, the contributions of the coating and of the bulk material to overall noise must be computed separately. For the coating, we consider (as in the case of semi-infinite mirrors) a new solution of the NavierCauchy equations in the region $\left[-\delta_{C}, 0\right]$; then the continuity of the displacements and the continuity of the pressures $\left(\Theta_{i j} n^{j}\right)$ at the interfaces $z=0$ and $z=-\delta_{C}$, allow us to completely determine the new solution. The energy density is

$$
\begin{aligned}
w(r, z)= & \frac{1}{2}\left[\lambda_{C} E(r, 0)^{2}+2 \mu_{c}\left(\left(E_{r r}(r, 0)+E_{\phi, \phi}(r, 0)\right)^{2}\right.\right. \\
& \left.\left.-2 E_{r r}(r, 0) E_{\phi, \phi}(r, 0)+E_{z z}(r, z)^{2}\right)\right]
\end{aligned}
$$

Due to the orthogonality of functions $J_{0}\left(k_{s} r\right)$, we get, after some algebra,

$$
U_{0, \text { coating }}=\frac{\delta_{C}}{2 \pi a^{2}} \frac{1+\sigma_{C}}{1-\sigma_{C}} \frac{1}{Y_{C}} \sum_{s} \frac{p_{s}^{2}}{\Delta_{s}^{2}} J_{0}\left(\zeta_{s}\right)^{2}\left[\left(1-2 \sigma_{C}\right) \Delta_{s}^{2}+\left(\frac{1+\sigma}{1+\sigma_{C}}\right)^{2} X_{s}^{2}\right]
$$

with the notation

$$
X_{s} \equiv(1-2 \sigma)\left(1-q_{s}\right)^{2}+4 q_{s} x_{s}^{2} .
$$

In the case of an homogeneous medium $\left(\lambda_{C}=\lambda, \mu_{C}=\mu\right)$, this reduces to

$$
U_{0, \text { coating,hom }}=\frac{\delta_{C}(1+\sigma)}{\pi a^{2} Y} \sum_{s} \frac{p_{s}^{2}}{\Delta_{s}^{2}} J_{0}\left(\zeta_{s}\right)^{2}\left[16 q_{s}^{2} x_{s}^{4}+(1-2 \sigma)\left(1-q_{s}\right)^{4}\right] .
$$

Note that the radial stress on the edge of the coating is

$$
\Theta_{r r}(a, 0)=-\frac{1}{\pi a^{2}} \sum_{s} p_{s} J_{0}\left(\zeta_{s}\right)\left[\sigma_{C}+\frac{Y_{C}(1+\sigma)}{Y\left(1+\sigma_{C}\right)} \frac{X_{s}}{\Delta_{s}}\right] .
$$

But we must add the contribution of the Saint-Venant correction. In the same way, we consider a new solution of the Navier-Cauchy equations connected to the preceding Saint-Venant correction. The components of the strain tensor in the coating are then

$$
\begin{gathered}
\Delta E_{r r}=\frac{1}{\pi a^{2} Y}[\sigma+6 S(1-\sigma)] \\
\Delta E_{\phi \phi}=\frac{1}{\pi a^{2} Y}[\sigma+6 S(1-\sigma)] \\
\Delta E_{z z}=-\frac{1}{\pi a^{2} Y} \frac{1}{Y_{C}\left(1-\sigma_{C}\right)}\left[2 Y_{C}(\sigma+6 S(1-\sigma))+Y\left(1+\sigma_{C}\right)\left(1-2 \sigma_{C}\right)\right] \\
\Delta E_{r z}=0 .
\end{gathered}
$$

After integrating the energy density over the volume $\pi a^{2} \delta_{C}$, one finds

$$
\Delta U_{\text {coating }}=\delta_{C} \frac{1+24 \sigma S+72(1-\sigma) S^{2}}{2 \pi a^{2} Y} \Omega_{2}
$$

with the notation

$$
\Omega_{2}=\frac{Y^{2}\left(1+\sigma_{C}\right)\left(1-2 \sigma_{C}\right)+2 Y_{C}^{2}[\sigma+6(1-\sigma) S]^{2}}{Y Y_{C}(1-\sigma)\left[1+24 \sigma S+72(1-\sigma) S^{2}\right]}
$$


Table 17: Comparison infinite/finite mirror Strain Energy (SE)

\begin{tabular}{lcc}
\hline 3 examples & coating SE $\left[\mathrm{J} \mathrm{N}^{-2}\right]$ & bulk SE $\left[\mathrm{J} \mathrm{N}^{-2}\right]$ \\
\hline Ex1: $\mathrm{LG}_{00}, w=2 \mathrm{~cm}$ & & \\
$\mathrm{U}_{\infty}$ & $2.10 \times 10^{-13}$ & $1.88 \times 10^{-10}$ \\
$\mathrm{U}$ & $2.38 \times 10^{-13}$ & $2.02 \times 10^{-10}$ \\
\hline Ex2: flat & $1.02 \times 10^{-14}$ & $3.95 \times 10^{-11}$ \\
$\mathrm{U}_{\infty}$ & $2.07 \times 10^{-14}$ & $3.28 \times 10^{-11}$ \\
$\mathrm{U}$ & & \\
\hline Ex3: $\mathrm{LG}_{55}, w=3.5 \mathrm{~cm}$ & $6.87 \times 10^{-15}$ & $2.68 \times 10^{-11}$ \\
$\mathrm{U}_{\infty}$ & $7.47 \times 10^{-15}$ & $8.64 \times 10^{-12}$ \\
$\mathrm{U}$ &
\end{tabular}

$\Omega_{2}$ takes the value one in the homogeneous case (when $\lambda_{C}=\lambda$ and $\mu_{C}=\mu$ ). Finally, $U_{\text {coating }}=$

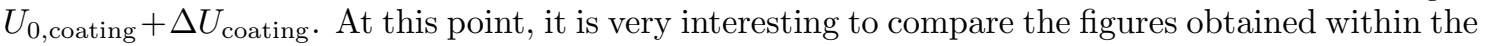
infinite medium approximation and the finite mirror formula. In Table 17, we give a few examples (in the homogeneous case) showing that the infinite mirror approximation is almost acceptable for peaked power distributions, but quite bad for wide beams, and especially for coating energy. Thus, optimization work based on infinite mirrors are, at minimum, questionable. 


\section{Thermoelastic Noise}

\subsection{Introduction}

The Brownian motion of matter inside the substrates is not the only cause of noise in the optical readout. There is another cause due to temperature fluctuations in a finite volume of material. These fluctuations are called thermodynamic and can couple with strain via the thermal dilatation constant $\alpha$, eventually producing random motions of the surface. A good way to model this kind of noise is to start from the general thermodynamic formulas detailed by Landau and Lifshitz [24] and use the Levin approach already presented. As in the preceding Section 8, we will consider the low frequency tail of the spectral density of the effective motion of the surface (i.e., the readout noise) as depending on the energy dissipated when the body is under a virtual pressure having the same profile as the optical beam and excited at low frequency. In this case, the spectral density is still of the form (Levin's formula)

$$
S_{x}(f)=\frac{4 k_{B} T}{\omega^{2}} W
$$

where $W$ is the average dissipated energy. For the standard thermal noise, we had $W=2 U \omega \Phi$ as average dissipated energy, $\Phi$ being a global loss angle and $U$ the static strain energy. But now $W$ must be interpreted as the energy dissipated via coupling of the strain with the temperature field in the bulk. Obviously, the temperature field itself depends on the strain field. Using the same approach as used in [28], we first solve the static linear elastic problem (done in the preceding Section 8), then we compute the resulting temperature field and use it to compute the dissipated energy. For computing the dissipated energy, we use the time dependence of the entropy. The variations of the entropy density $S$ are related to the heat flux $\vec{q}$ by requiring conservation of the energy in the body

$$
T \frac{\partial S}{\partial t}=-\operatorname{div}(\vec{q})
$$

where $\vec{q}=-K \operatorname{grad} T, K$ being the thermal conductivity of the material (cf. Landau and Lifshitz [24]). Or, as well,

$$
\frac{\partial S}{\partial t}=-\frac{1}{T} \operatorname{div}(\vec{q})
$$

Therefore, total entropy variation in the body is

$$
\frac{d S_{\mathrm{tot}}}{d t}=-\int \frac{1}{T} \operatorname{div}(\vec{q}) d V
$$

where the integral is extended to the whole body. This is

$$
\frac{d S_{\text {tot }}}{d t}=-\int \operatorname{div} \frac{\vec{q}}{T} d V+\int \vec{q} \cdot \operatorname{grad}\left(\frac{1}{T}\right) d V .
$$

Neglecting the heat flow at the surface of the body, the first integral vanishes, and we have

$$
\frac{d S_{\mathrm{tot}}}{d t}=-\int \frac{1}{T^{2}} \vec{q} \cdot \operatorname{grad} T d V .
$$

But using the definition of $\vec{q}$, this is

$$
\frac{d S_{\mathrm{tot}}}{d t}=\int \frac{K}{T^{2}}(\operatorname{grad} T)^{2} d V
$$

so that the energy variation is

$$
W=T \frac{d S_{\mathrm{tot}}}{d t}=\int \frac{K}{T}(\operatorname{grad} T)^{2} d V .
$$


We shall say now that the temperature gradient field is caused by the small deformations of the body that we computed earlier, while $T$ is the mean temperature. This becomes

$$
W=T \frac{d S_{\mathrm{tot}}}{d t}=\frac{K}{T} \int(\operatorname{grad} \delta T)^{2} d V .
$$

where we have replaced $T$ by a $\delta T$ in the gradient for more clarity. On the other hand, it is well known (cf. Landau-Lifshitz) that the total entropy is the sum of two terms, one being the entropy in the reference state, and a second one proportional to the trace $E$ of the strain tensor

$$
S=S_{0}+\nu E
$$

$\nu$ being the thermoelastic coefficient, so that there is, in the bulk material, a power source given by

$$
P=T \frac{d S}{d t}=\nu T \frac{d E}{d t}
$$

where $E$ is the trace of $E_{i k}$. The resulting temperature field obeys the Heat (Fourier) equation

$$
\left(\rho C \partial_{t}-K \Delta\right) \delta T=\nu T \frac{d E}{d t} .
$$

The trace of the strain tensor $E_{i k}$ found in the preceding Section 8 is, in any case, a harmonic function, so that there is a trivial solution

$$
\delta T=\frac{\nu T}{\rho C} E .
$$

The boundary conditions (null heat flux on the surfaces) are considered satisfied in time average ( $\delta T$ is assumed oscillating at a few tens of $\mathrm{Hz}$ ). In fact, they are exactly satisfied on the circular edge of the mirror. Now we reach the relevant equation for the dissipated energy

$$
W=\frac{K \nu^{2} T}{\rho^{2} C^{2}} \int(\operatorname{grad} E)^{2} d V
$$

$\nu$ is related to the linear dilatation coefficient $\alpha$ by

$$
\nu=\frac{\alpha Y}{1-2 \sigma},
$$

where $Y$ is Young's modulus and $\sigma$ the Poisson ratio. Finally,

$$
W=K T\left[\frac{\alpha Y}{(1-2 \sigma) \rho C}\right]^{2} \int(\operatorname{grad} E)^{2} d V
$$

(see [28]). We have, after the preceding Section 8 on standard thermal noise, everything we need to compute $W$.

\subsection{Case of infinite mirrors}

Let us recall the results obtained in the preceding chapter on standard thermal noise. Under beam pressure, the displacement vector is

$$
u_{r}(r, z)=\int_{0}^{\infty} \alpha(k)[k z-1+2 \sigma] \exp (-k z) J_{1}(k z) k d k
$$




$$
u_{z}(r, z)=\int_{0}^{\infty} \alpha(k)[k z+2-2 \sigma] \exp (-k z) J_{0}(k z) k d k
$$

so that

$$
E(r, z)=\operatorname{div} \vec{u}(r, z)=-2(1-2 \sigma) \int_{0}^{\infty} \alpha(k) \exp (-k z) J_{0}(k z) k^{2} d k .
$$

The function $u(k)$ is determined by the virtual pressure distribution $I(r)$ (normalized beam intensity). Namely,

$$
\alpha(k)=-\frac{1+\sigma}{Y} \frac{\tilde{I}(k)}{k},
$$

where $\tilde{I}(k)$ is the Hankel transform of $I(r)$. As a result,

$$
E(r, z)=-\frac{2(1-2 \sigma)(1+\sigma)}{Y} \int_{0}^{\infty} \tilde{I}(k) \exp (-k z) J_{0}(k r) k d k,
$$

which shows, in passing, that

$$
E(r, 0)=-\frac{2(1-2 \sigma)(1+\sigma)}{Y} I(r)
$$

Thus, wee can already foresee that in the case of an ideally flat beam the gradient will involve Dirac distributions; therefore, the volume integration of its square will be problematic. Let us compute the gradient of $E$ :

$$
\begin{aligned}
& \frac{\partial E}{\partial r}=\frac{2(1-2 \sigma)(1+\sigma)}{Y} \int_{0}^{\infty} \tilde{I}(k) \exp (-k z) J_{1}(k r) k^{2} d k \\
& \frac{\partial E}{\partial z}=\frac{2(1-2 \sigma)(1+\sigma)}{Y} \int_{0}^{\infty} \tilde{I}(k) \exp (-k z) J_{0}(k r) k^{2} d k .
\end{aligned}
$$

Now, using the closure relation,

$$
\int_{0}^{\infty} J_{\nu}(k r) J_{\nu}\left(k^{\prime} r\right) r d r=\frac{\delta\left(k-k^{\prime}\right)}{k}
$$

for $\nu=0,1$. It is now possible to carry out the volume integration:

$$
2 \pi \int_{0}^{\infty} r d r \int_{0}^{\infty} d z(\operatorname{grad} E)^{2}=8 \pi \frac{(1-2 \sigma)^{2}(1+\sigma)^{2}}{Y^{2}} \int_{0}^{\infty} \tilde{I}(k)^{2} k^{2} d k
$$

so that

$$
W=8 \pi \frac{K T \alpha^{2}(1+\sigma)^{2}}{\rho^{2} C^{2}} \varpi_{2}
$$

with

$$
\varpi_{2}=\int_{0}^{\infty} \tilde{I}(k)^{2} k^{2} d k
$$

This expression shows that the function $\tilde{I}(k)$ must have an asymptotic evanescence strictly faster than $k^{-3 / 2}$ for the integral to converge. This is a strong requirement on the Hankel transform of the pressure distribution. 
Table 18: Some numerical values of $g_{2, n, m}$

\begin{tabular}{ll|cccccc}
\hline & $\mathrm{m}$ & 0 & 1 & 2 & 3 & 4 & 5 \\
$\mathrm{n}$ & & & & & & \\
\hline 0 & & 1 & .75 & .64 & .57 & .53 & .49 \\
1 & & .44 & .39 & .36 & .33 & .31 & .30 \\
2 & & .33 & .31 & .29 & .27 & .26 & .25 \\
3 & & .28 & .26 & .25 & .24 & .23 & .22 \\
4 & & .24 & .23 & .22 & .21 & .21 & .20 \\
5 & & .22 & .21 & .20 & .20 & .19 & .19 \\
\hline
\end{tabular}

\subsubsection{Gaussian beams}

For a Laguerre-Gauss mode $\mathrm{LG}_{n, m}$ of width parameter $w$, we have seen that

$$
\tilde{I}_{n, m}(k)=\frac{1}{2 \pi} \mathrm{e}^{-y} L_{m}(y) L_{m+n}(y) \quad\left(y \equiv k^{2} w^{2} / 8\right)
$$

giving

$$
\varpi_{2, n, m}=\frac{1}{2 \pi \sqrt{\pi} w^{3}} g_{2, n, m}
$$

where $g_{2, n, m}$ are numerical factors (see Table 18).

Thus,

$$
\int(\operatorname{grad} E)^{2} d V=\frac{4(1-2 \sigma)^{2}(1+\sigma)^{2}}{\sqrt{\pi} Y^{2} w^{3}} g_{2, n, m}
$$

so that the spectral density of thermoelastic noise is, using Equations (9.1) and (9.16),

$$
S_{x}(f)=\frac{4 k_{B} K T^{2} \alpha^{2}(1+\sigma)^{2}}{\pi^{2} \sqrt{\pi} \rho^{2} C^{2} f^{2} w^{3}} g_{2, n, m}
$$

This result was found (in the case of $n=m=0$ ) first by Braginsky et al. [7] using their own formalism, then by Liu et al. [28], using our approach. For silica parameters and for Ex1 (the $\mathrm{LG}_{0,0}$ mode with $w=2 \mathrm{~cm}$ ), one finds

$$
\varpi_{2,0,0}=11,224 \mathrm{~m}^{-3}
$$

and

$$
S_{x}(f)^{1 / 2}=8.53 \times 10^{-20}\left[\frac{1 \mathrm{~Hz}}{f}\right] \mathrm{mHz}^{-1 / 2},
$$

which is lower than the standard thermal noise, but still significant. For Ex3 (the $\mathrm{LG}_{5,5}$ mode with $w=3.5 \mathrm{~cm}$ ), we have

$$
\varpi_{2,5,5}=398 \mathrm{~m}^{-3}
$$

$$
S_{x}(f)^{1 / 2}=1.61 \times 10^{-20}\left[\frac{1 \mathrm{~Hz}}{f}\right] \mathrm{mHz}^{-1 / 2} .
$$




\subsubsection{Flat beams}

If we now consider a flat beam modeled by its ideal representation

$$
I(r)=\left\{\begin{array}{l}
1 / \pi b^{2} \quad(r<b) \\
0 \quad(r \geq b)
\end{array}\right.
$$

we have the Hankel transform

$$
\tilde{I}(k)=\frac{J_{1}(k b)}{\pi k b},
$$

which shows that the requirement on the decreasing rate for large $k$ is not fulfilled, $J_{\nu}(k)$ having asymptotic behavior in $k^{-1 / 2}$, just below the limit. Therefore, it is impossible to use the crude flat model, the integral $\varpi_{2}$ being divergent. If we want to have an evaluation, we must carry out a numerical integration with the mesa intensity profile. We find (for our particular model)

$$
\varpi_{2} \sim 77 \mathrm{~m}^{-3}
$$

seeming to indicate a strong reduction factor of the SD of noise, namely 0.44 with respect to Ex3. This result is due to the fact that the $\mathrm{LG}_{5,5}$ mode has a number of rings causing many local gradients. This was pointed out by Agresti ([2]) in the case of the $\mathrm{LG}_{0,5}$ mode. Anyway this mode is unwanted, as well as other $\mathrm{LG}_{0, m}$ modes, because we wish to avoid sharp central power peaks .

\subsubsection{Thermoelastic noise in the coating}

We apply the same strategy for all coating calculations. The gradient of the trace of the strain tensor can be integrated on the surface $z=0$ giving

$$
\int_{0}^{\infty} \nabla E^{2} r d r=\frac{8(1+\sigma)^{2}(1-2 \sigma)^{2}}{Y^{2}} \Omega_{3} \varpi_{3}
$$

with

$$
\Omega_{3} \equiv \frac{1}{2}\left[\left(\frac{(1-\sigma)\left(1-2 \sigma_{C}\right)}{\left(1-\sigma_{C}\right)(1-2 \sigma)}\right)^{2}+\frac{1}{4\left(1-\sigma_{C}\right)^{2}}\left[1-2 \sigma_{C}+\frac{Y}{Y_{C}} \frac{\left(1+\sigma_{C}\right)\left(1-2 \sigma_{C}\right)}{(1+\sigma)\left(1-2 \sigma_{C}\right)}\right]^{2}\right]
$$

$\left(\Omega_{3}\right.$ takes the value one when $\left.Y=Y_{C}, \sigma=\sigma_{C}\right)$. Contrary to $\Omega_{1}$, which is not so sensitive to parameters, $\Omega_{3}$ can take values quite different from one. For instance, if we assume the parameters of fused silica for the substrate, and $\left(Y_{C} \sim 1.4 \times 10^{11} \mathrm{~N} \mathrm{~m}^{-2}, \sigma_{C} \sim 0.23\right)$ for the coating, we have $\Omega_{3} \sim 0.59 . \varpi_{3}$ has the following definition

$$
\varpi_{3} \equiv \int_{0}^{\infty} \tilde{I}(k)^{2} k^{3} d k
$$

so that the energy $W$ is (taking into account special values for the coating material)

$$
W=16 \pi K_{C} T\left(\frac{\alpha_{C}\left(1+\sigma_{C}\right)}{\rho_{C} C_{C}}\right)^{2} \Omega_{3} \varpi_{3} \delta_{C}
$$

In the case of $\mathrm{LG}_{n, m}$ modes, we obtain

$$
\varpi_{3, n, m}=\frac{2}{\pi^{2} w^{4}} g_{3, n, m},
$$

where $g_{3, n, m}$ are numerical factors, the first ones being given by Table 19 .

It seems clear that, as already mentioned, the modes $\mathrm{LG}_{0, m}$, having a sharp peak on the axis, become worse and worse as the order $m$ increases. On the other hand, the reduction factor for the noise in the best cases is much less than for the Brownian thermal noise. 
Table 19: Some numerical values of $g_{3, n, m}$

\begin{tabular}{|c|c|c|c|c|c|c|}
\hline $\mathrm{n}^{\mathrm{m}}$ & 0 & 1 & 2 & 3 & 4 & 5 \\
\hline 0 & 1 & 1.5 & 1.72 & 1.86 & 1.96 & 2.05 \\
\hline 1 & .50 & .81 & .98 & 1.10 & 1.20 & 1.27 \\
\hline 2 & .37 & .62 & .77 & .88 & .96 & 1.03 \\
\hline 3 & .31 & .53 & .66 & .76 & .83 & .90 \\
\hline 4 & .27 & .46 & .58 & .67 & .75 & .81 \\
\hline 5 & .25 & .42 & .53 & .61 & .68 & .74 \\
\hline
\end{tabular}

Table 20: Some values of $\varpi_{n}$

\begin{tabular}{llllll}
\hline $\mathrm{n}$ & $\mathrm{LG}_{00} w=2 \mathrm{~cm}$ & $\mathrm{LG}_{55} w=3.5 \mathrm{~cm}$ & flat $b=9.1 \mathrm{~cm}$ & mesa $b_{f}=10.7 \mathrm{~cm}$ & units \\
\hline 0 & 2.245 & 0.321 & 0.473 & 0.426 & $\mathrm{~m}^{-1}$ \\
1 & 126.65 & 4.13 & 6.12 & 4.52 & $\mathrm{~m}^{-2}$ \\
2 & $1.122 \times 10^{4}$ & 398 & $*$ & 76.7 & $\mathrm{~m}^{-3}$ \\
3 & $1.27 \times 10^{6}$ & $10^{5}$ & $*$ & 1800 & $\mathrm{~m}^{-4}$ \\
\hline
\end{tabular}

\subsubsection{Scaling laws}

This section offers an opportunity to summarize the various coefficients encountered in the parts of this noise study. Several authors (see [29] for his discussion) have remarked on the dependence of the various noises encountered on the integrals we have denoted $\varpi_{m},\{m \in \mathbb{N}\}$.

$$
\varpi_{m}=\int_{0}^{\infty} \tilde{I}(k) k^{m} d k
$$

- Brownian noise, substrate: $\varpi_{0}$

- Brownian noise, coating: $\varpi_{1}$

- Thermoelastic noise, substrate: $\varpi_{2}$

- Thermoelastic noise, coating: $\varpi_{3}$. However, in this case there is a more refined analysis [29], taking into account the heat flow. Attention must be paid to this theory (see also [17, 8]). However, the approximate character of the semi-infinite-mirror approach reduces its practical interest.

We have given these integrals in the case of different $\mathrm{LG}_{m n}$ modes. In particular, Table 20 gives the values for our four examples. These can be used to derive figures of merit.

\subsubsection{Numerical results}

We give briefly some figures regarding our three reference situations. We take the parameter of fused silica for the substrate, and the parameters of $\mathrm{TA}_{2} \mathrm{O}_{5}$ for the coating ([36]), namely, $Y_{C}=1.410^{11} \mathrm{~Pa}, \sigma_{C}=0.23, \rho_{C} \times C_{C}=2.1 \times 10^{6} \mathrm{~J} / \mathrm{m}^{3} / \mathrm{K}$. The thickness of the coating is assumed to be $25 \mu \mathrm{m}$. For a $\mathrm{TEM}_{0,0}$ mode of waist $2 \mathrm{~cm}$, we obtain

- Spectral density of thermoelastic noise in the substrate:

$$
S_{x}(f)^{1 / 2}=8.53 \times 10^{-20} \mathrm{mHz}^{-1 / 2}\left[\frac{1 \mathrm{~Hz}}{f}\right] .
$$


Spectral density of noise in the coating:

$$
S_{x}(f)^{1 / 2}=1.32 \times 10^{-19} \mathrm{mHz}^{-1 / 2}\left[\frac{1 \mathrm{~Hz}}{f}\right] .
$$

We see that the large difference in parameters overcompensates for the difference in volume. For an $\mathrm{LG}_{55}$ mode of waist $3.5 \mathrm{~cm}$ :

- Spectral density of thermoelastic noise in the substrate:

$$
S_{x}(f)^{1 / 2}=1.61 \times 10^{-20} \mathrm{mHz}^{-1 / 2}\left[\frac{1 \mathrm{~Hz}}{f}\right] .
$$

Spectral density of noise in the coating:

$$
S_{x}(f)^{1 / 2}=3.71 \times 10^{-20} \mathrm{mHz}^{-1 / 2}\left[\frac{1 \mathrm{~Hz}}{f}\right] .
$$

The reduction factor is about five for the substrate and only 3.5 for the coating. We see that the large difference in parameters overcompensates for the difference in volume. For a mesa mode:

- Spectral density of thermoelastic noise in the substrate:

$$
S_{x}(f)^{1 / 2}=7.05 \times 10^{-21} \mathrm{mHz}^{-1 / 2}\left[\frac{1 \mathrm{~Hz}}{f}\right] .
$$

- Spectral density of noise in the coating:

$$
S_{x}(f)^{1 / 2}=4.99 \times 10^{-21} \mathrm{mHz}^{-1 / 2}\left[\frac{1 \mathrm{~Hz}}{f}\right] .
$$

The reduction factor with respect to Ex1 is about 12 for the substrate and 26 for the coating. This kind of mode is obviously the best regarding this kind of noise.

\subsection{Case of finite mirrors}

In the case of finite mirrors, the model developed for standard thermal noise provides the explicit expressions for the trace $E$ of the strain tensor

$$
E(r, z)=E_{0}(r, z)+\Delta E(r, z)
$$

with

$$
E_{0}(r, z)=-\frac{2(1-2 \sigma)(1+\sigma)}{\pi a^{2} Y} \sum_{s>0} \frac{p_{s}}{\Delta_{s}} J_{0}\left(\zeta_{s} r / a\right)\left[Q_{s} \mathrm{e}^{-\zeta_{s} z / a}-R_{s} \mathrm{e}^{\zeta_{s} z / a}\right],
$$

where $p_{s}$ are the Fourier-Bessel coefficients of the pressure distribution, and $\Delta_{s}, q_{s}, x_{s}, Q_{s}$ and $R_{s}$ have been defined in the preceding Section 8. Moreover,

$$
\Delta E(r, z)=-(1-2 \sigma) \frac{1}{\pi a^{2} Y}[1-12 S-(1-24 S) z / h]
$$

(see Equation (8.105) for $S$ ), so that the gradient of $E$ is

$$
\begin{gathered}
\frac{\partial E_{0}}{\partial r}=\frac{2(1-2 \sigma)(1+\sigma)}{\pi a^{3} Y} \sum_{s>0} \frac{p_{s} \zeta_{s}}{\Delta_{s}} J_{1}\left(k_{s} r\right)\left[Q_{s} \mathrm{e}^{-k_{s} z}-R_{s} \mathrm{e}^{k_{s} z}\right] \\
\frac{\partial E_{0}}{\partial z}=\frac{2(1-2 \sigma)(1+\sigma)}{\pi a^{3} Y} \sum_{s>0} \frac{p_{s} \zeta_{s}}{\Delta_{s}} J_{0}\left(k_{s} r\right)\left[Q_{s} \mathrm{e}^{-k_{s} z}+R_{s} \mathrm{e}^{k_{s} z}\right] \\
\frac{\partial \Delta E}{\partial z}=\frac{1-2 \sigma}{\pi a^{2} h Y}(1-24 S) .
\end{gathered}
$$




\subsubsection{Case of the bulk material}

Owing to the orthogonality relations of $J_{\nu}\left(\zeta_{s} r / a\right)$, we get

$$
\int\left(\overrightarrow{\operatorname{grad}} E_{0}\right)^{2} d V=\frac{4(1-2 \sigma)^{2}(1+\sigma)^{2}}{\pi a^{3} Y^{2}} \sum_{s>0} W_{s}
$$

where

$$
\begin{aligned}
W_{s}= & \frac{p_{s}^{2} \zeta_{s}}{\Delta_{s}^{2}} J_{0}\left(\zeta_{s}\right)^{2}\left(1-q_{s}\right) \\
& \times\left[\left(1-q_{s}\right)\left(1-q_{s}^{2}\right)+8 q_{s} x_{s}\left(1-q_{s}+x_{s}\right)\right]
\end{aligned}
$$

and, obviously,

$$
\int(\operatorname{grad} \Delta E)^{2} d V=\frac{(1-2 \sigma)^{2}}{\pi a^{2} h Y^{2}}(1-24 S)^{2}
$$

(N.B. $\operatorname{grad} \Delta E$ and $\overrightarrow{\operatorname{grad}} E_{0}$ are orthogonal in the $r$ integration). We have, finally,

$$
W=\frac{4 K T \alpha^{2}}{\pi a^{3} \rho^{2} C^{2}}\left[(1+\sigma)^{2} \sum_{s>0} W_{s}+(1-24 S)^{2} \frac{a}{4 h}\right] .
$$

And for the spectral density,

$$
S_{x}(f)=\frac{4 k_{B} K T^{2} \alpha^{2}}{\pi^{3} a^{3} \rho^{2} C^{2} f^{2}}\left[(1+\sigma)^{2} \sum_{m>0} W_{s}+(1-24 S)^{2} \frac{a}{4 h}\right] .
$$

For Gaussian beams, we substitute the $p_{s}$ 's in the preceding formulas. For the parameters corresponding to Virgo input mirrors $\left(\mathrm{LG}_{0,0}, w=2 \mathrm{~cm}\right)$ we find

$$
S_{x}^{1 / 2}(f)=8.83 \times 10^{-20} \mathrm{mHz}^{-1 / 2}\left[\frac{1 \mathrm{~Hz}}{f}\right] .
$$

For the flat beam, $b=9.1 \mathrm{~cm}$,

$$
S_{x}^{1 / 2}(f)=1.87 \times 10^{-20} \mathrm{mHz}^{-1 / 2}\left[\frac{1 \mathrm{~Hz}}{f}\right] .
$$

For the mesa beam, $b_{f}=10.7 \mathrm{~cm}$,

$$
S_{x}^{1 / 2}(f)=1.49 \times 10^{-20} \mathrm{mHz}^{-1 / 2}\left[\frac{1 \mathrm{~Hz}}{f}\right] .
$$

For the $\mathrm{LG}_{5,5}$ beam, $w=3.5 \mathrm{~cm}$,

$$
S_{x}^{1 / 2}(f)=1.72 \times 10^{-20} \mathrm{mHz}^{-1 / 2}\left[\frac{1 \mathrm{~Hz}}{f}\right] .
$$

In Figures 63, 64, 65, and 66, one can see the distribution of $(\overrightarrow{g r a d} E)^{2}$ in Ex1, Ex2, the mesa beam, and Ex3, respectively.

In the case of the flat beam, one should note the peaks at the location of the sharp edges of the intensity distribution. This was the cause of the divergence of the infinite mirror approach. However, the estimation for the flat and mesa beams are not very different. 


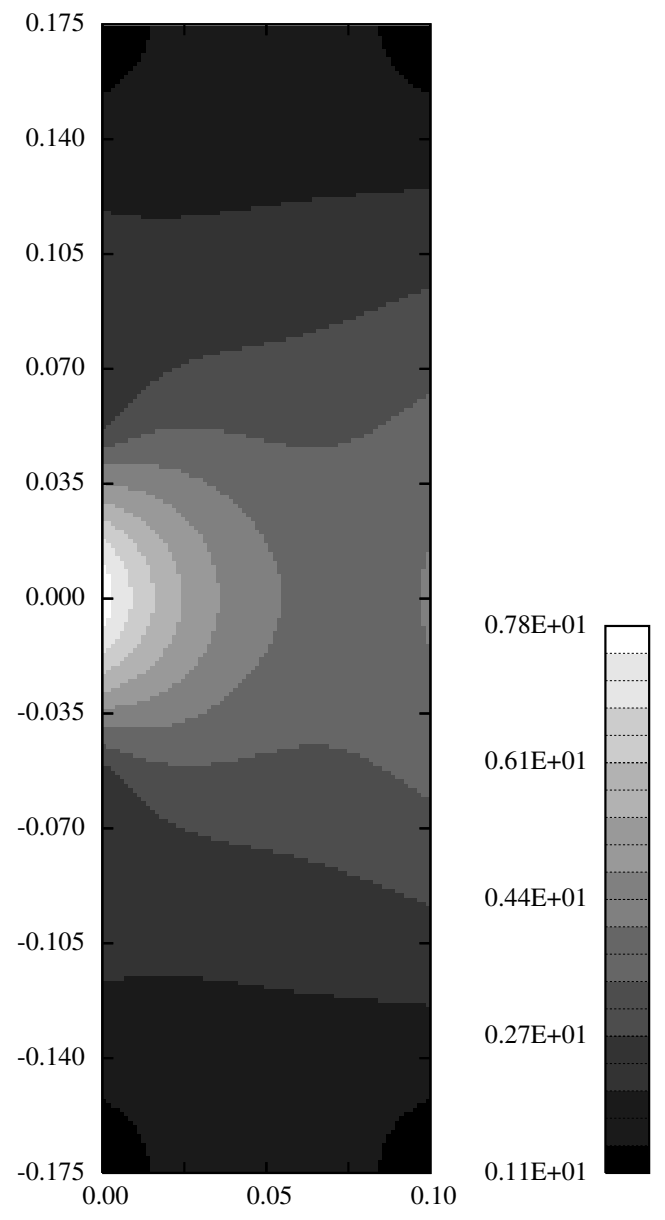

Figure 63: Distribution of $(\vec{\nabla} E)^{2}$ in the case of a $\mathrm{LG}_{0,0}$ mode $(w=2 \mathrm{~cm})$. (Logarithmic scale, arbitrary units) 


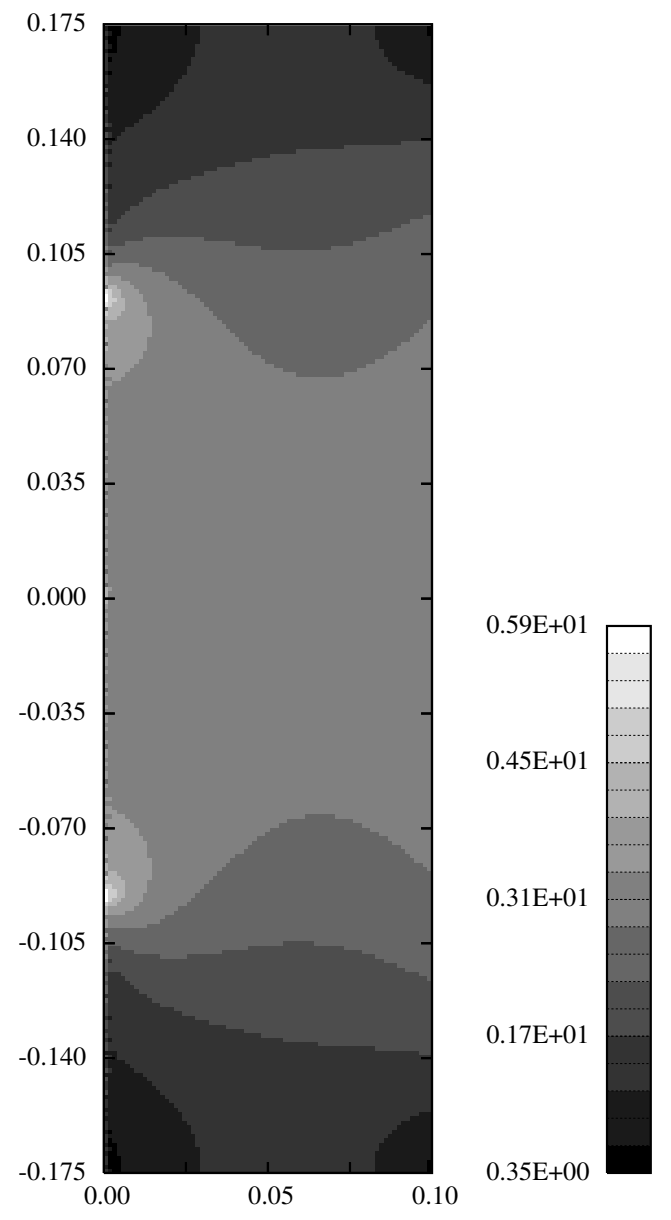

Figure 64: Distribution of $(\vec{\nabla} E)^{2}$ in the case of a flat mode $(b=9.1 \mathrm{~cm})$. (Logarithmic scale, arbitrary units) 


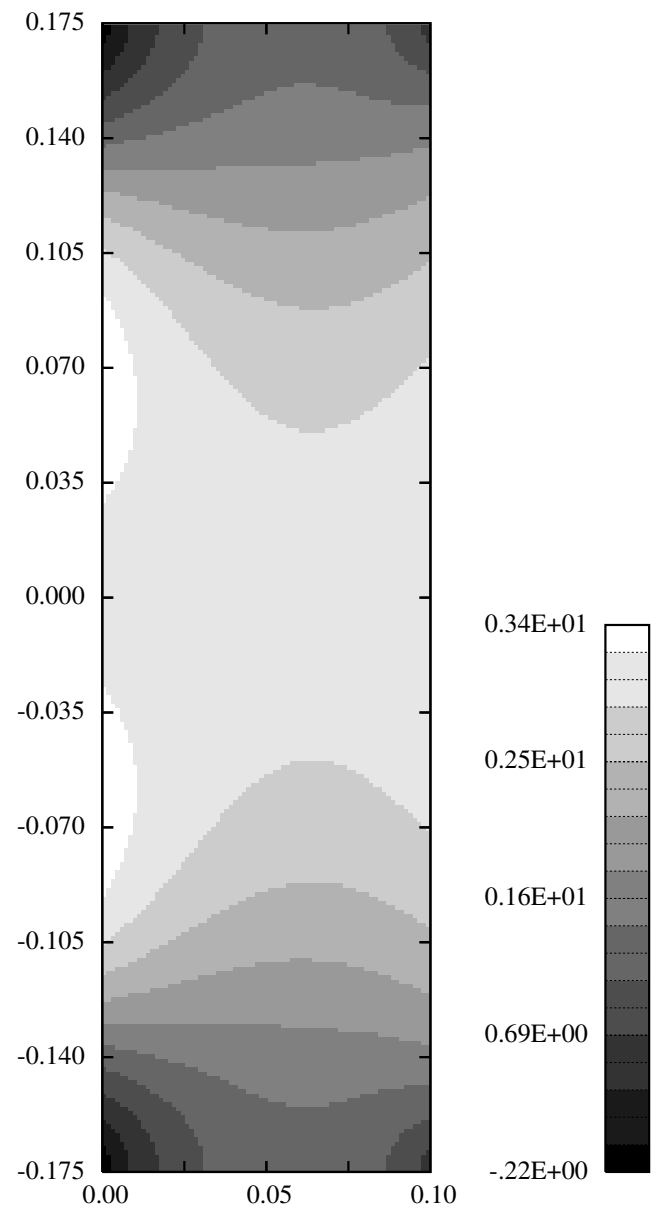

Figure 65: Distribution of $(\vec{\nabla} E)^{2}$ in the case of a mesa mode $\left(b_{f}=10.7 \mathrm{~cm}\right)$. (Logarithmic scale, arbitrary units) 


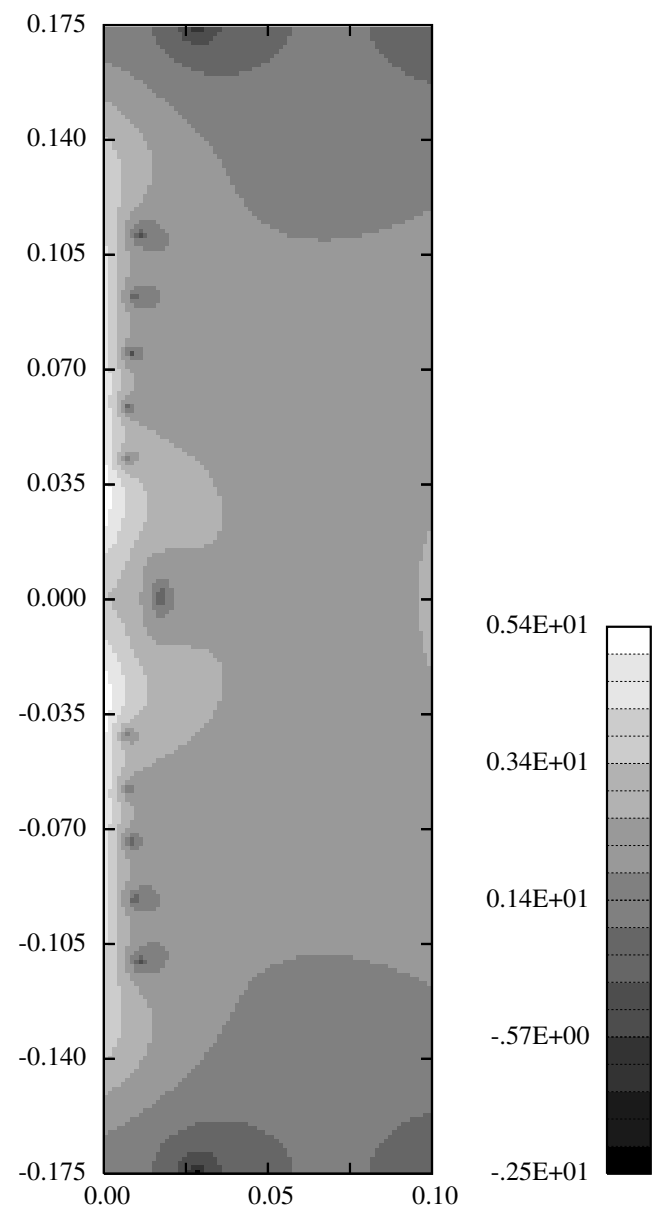

Figure 66: Distribution of the square gradient of the trace of the strain tensor in the case of an $L_{G_{5,5}}$ mode $(w=3.5 \mathrm{~cm})$. (Logarithmic scale, arbitrary units) 


\subsubsection{Case of the coatings}

The components of the gradient of the trace of the strain tensor on the reflecting surface can be obtained from the model developed for the Brownian thermal noise. We consider a new solution of the Navier-Cauchy equations, matched to the bulk solution at $z=0$, and depending on specific parameters $Y_{C}$ and $\sigma_{C}$. The components of the gradient are as follows, with the notation already introduced.

$$
\begin{gathered}
\frac{\partial E}{\partial r}=\frac{(1+\sigma)\left(1-2 \sigma_{C}\right)}{\pi a^{3} Y\left(1-\sigma_{C}\right)} \sum_{s} \frac{p_{s} \zeta_{s}}{\Delta_{s}}\left[2(1-\sigma)\left(1-q_{s}^{2}+4 q_{s} x_{s}\right)\right] J_{1}\left(k_{s} r\right) \\
\frac{\partial E}{\partial z}=\frac{(1+\sigma)\left(1-2 \sigma_{C}\right)}{\pi a^{3} Y\left(1-\sigma_{C}\right)} \sum_{s} \frac{p_{s} \zeta_{s}}{\Delta_{s}}\left[\frac{Y\left(1+\sigma_{C}\right)}{Y_{C}(1+\sigma)} \Delta_{s}+X_{s}\right] J_{0}\left(k_{s} r\right),
\end{gathered}
$$

so that a volume integration gives

$$
2 \pi \delta_{C} \int_{0}^{a}(\vec{\nabla} E)^{2} r d r=\delta_{c} \frac{(1+\sigma)^{2}\left(1-2 \sigma_{C}\right)^{2}}{\pi a^{4} Y^{2}\left(1-\sigma_{C}\right)^{2}} \sum_{s} \frac{p_{s}^{2} \zeta_{s}^{2} J_{0}\left(\zeta_{s}\right)^{2}}{\Delta_{s}^{2}} V_{s}
$$

with

$$
V_{s} \equiv 4\left(1-\sigma^{2}\right)^{2}\left(1-q_{s}^{2}+4 q_{s} x_{s}\right)^{2}+\left[\frac{Y\left(1+\sigma_{C}\right)}{Y_{C}(1+\sigma)} \Delta_{s}+X_{s}\right]^{2} .
$$

The Saint-Venant correction to $E$ only has a $z$ derivative, so that

$$
2 \pi \delta_{C} \int_{0}^{a}(\vec{\nabla} \Delta E)^{2} r d r=\delta_{C} \frac{4\left(1-2 \sigma_{C}\right)^{2}[\sigma+12 S(1-\sigma)]^{2}}{\pi a^{2} h^{2} Y^{2}\left(1-\sigma_{C}\right)^{2}} .
$$

And the global result is

$$
\begin{aligned}
W= & K_{C} T\left(\frac{\alpha}{\rho_{C} C_{C}}\right)^{2} \frac{\delta_{C}}{\pi a^{4}\left(1-\sigma_{C}\right)^{2}}\left[(1+\sigma)^{2} \sum_{s} \frac{p_{s}^{2} \zeta_{s}^{2} J_{0}\left(\zeta_{s}\right)^{2}}{\Delta_{s}^{2}} V_{s}\right. \\
& \left.+\frac{4 a^{2}}{h^{2}}(\sigma+12 S(1-\sigma))^{2}\right] .
\end{aligned}
$$

\subsubsection{Numerical results}

With the coating parameters already given, we get the following results for the coating noise. For the $\mathrm{LG}_{00}$ mode with $w=2 \mathrm{~cm}$ waist:

$$
S_{x}^{1 / 2}(f)=1.54 \times 10^{-19} \mathrm{mHz}^{-1 / 2}\left[\frac{1 \mathrm{~Hz}}{f}\right] .
$$

For the $\mathrm{LG}_{55}$ mode with $w=3.5 \mathrm{~cm}$ :

$$
S_{x}^{1 / 2}(f)=4.33 \times 10^{-20} \mathrm{mHz}^{-1 / 2}\left[\frac{1 \mathrm{~Hz}}{f}\right] .
$$

And for the mesa mode:

$$
S_{x}^{1 / 2}(f)=8.66 \times 10^{-21} \mathrm{mHz}^{-1 / 2}\left[\frac{1 \mathrm{~Hz}}{f}\right] .
$$

We see how the infinite mirror model underestimates the noise by a factor of two for the last case. 


\section{Generation of High Order Modes}

Several methods have been proposed to generate LG modes. We discuss here a particular method based on fiber optics. It is known that cylindrical fibers having three layers (core plus two claddings) can exhibit modes having an annular structure more or less analogous to an LG mode. Consider a fiber having a core of radius $r_{C}$, a cladding confined to the zone $r_{C}<r<r_{G}$ and an extra cladding for $r>r_{G}$. The latter can be assumed infinite for the guided modes, which have an evanescent behavior in that region, so that the external radius is never reached by the light. The refractive indices are $n_{C}$ in the core, $n_{G}$ in the first cladding and $n_{\text {ext }}$ in the external cladding. We assume $n_{C}>n_{G}>n_{\text {ext }}$. Assuming a wave of the form ( $p$ is any integer and $\left.k \equiv 2 \pi / \lambda\right)$

$$
\mathcal{E}(r, z)=\mathrm{e}^{i k n_{\mathrm{eff}} z} E_{p}(r) \mathrm{e}^{i p \varphi},
$$

where $\mathcal{E}$ is any component of the optical wave and $n_{\text {eff }}$ is a parameter depending on the fiber geometry (radii and indices). The wave equation reduces to

$$
\left[\partial_{r}^{2}+\frac{1}{r} \partial_{r}-\frac{p^{2}}{r^{2}}+k^{2}\left(n^{2}-n_{\mathrm{eff}}^{2}\right)\right] E_{p}(r)=0 .
$$

There exist families of modes depending on the value of $n_{\text {eff }}$ compared to $n_{c}, n_{G}$, and $n_{\text {ext }}$. Modes such that $n_{C}>n_{\mathrm{eff}}>n_{G}$ are called core modes. Modes such that $n_{C}>n_{G}>n_{\mathrm{eff}}>n_{\mathrm{ext}}$ are called cladding modes. We are interested in cladding modes because the central part of the beam is vanishing in this case (as in an $\mathrm{LG}_{n, n}, n \neq 0$ mode). A further (realistic) assumption is that the indices are slightly different. In this case, the weak guidance model holds, leading to linearly polarized modes called LP. Solving Equation (10.2) leads to a wave of the form

$$
E_{p}(r)=\left\{\begin{array}{l}
A J_{p}\left(U r / r_{C}\right) \quad\left(r<r_{C}\right) \\
B J_{p}\left(U_{g} r / r_{G}\right)+B Y_{p}\left(U_{g} r / r_{G}\right) \quad\left(r_{C} \leq r<r_{G}\right) \\
D K_{p}\left(W r / r_{C}\right) \quad\left(r>r_{G}\right)
\end{array}\right.
$$

with the following notation:

$$
U=k r_{C} \sqrt{n_{C}^{2}-n_{\mathrm{eff}}^{2}}, \quad U_{g}=k r_{G} \sqrt{n_{G}^{2}-n_{\mathrm{eff}}^{2}}, \quad W=k r_{G} \sqrt{n_{\mathrm{eff}}^{2}-n_{\mathrm{ext}}^{2}} .
$$

$J_{p}$ and $Y_{p}$ are Bessel functions of the first and second kind, respectively. $K_{p}$ is a modified Bessel function of the second kind. The structure of the solution was dictated by the following considerations: The solution must be regular at $r=0$, (no $Y_{p}$ contribution in the core), the solution must be evanescent in the external cladding (no $I_{p}$ contribution there). Now the arbitrary constants $A, B$, and $C$ can be reduced to one after taking into account the boundary conditions. The boundary conditions require continuity of the components of the field tangential to the cylindrical interfaces at $r_{C}$, and $r_{G}$. In the weak guidance model, this is equivalent to requiring a smooth solution at the interfaces and smooth derivatives. Only discrete values of $n_{\mathrm{eff}}$ make it possible, and these discrete values determine families of modes. The central issue of the guide theory is thus to find these values. If we adopt the following notation $\left(S \equiv r_{G} / r_{C}\right)$ :

$$
\begin{gathered}
M\left(n_{\mathrm{eff}}\right)=U_{g} Y_{p+1}\left(U_{g}\right) K_{p}(W)-W K_{p+1}(W) Y_{p}\left(U_{g}\right) \\
N\left(n_{\mathrm{eff}}\right)=U J_{p+1}(U) J_{p}\left(U_{g} / S\right)-\frac{U_{g}}{S} J_{p+1}\left(U_{g} / S\right) J_{p}(U) \\
P\left(n_{\mathrm{eff}}\right)=U_{g} J_{p+1}\left(U_{g}\right) K_{p}(W)-W K_{p+1}(W) J_{p}\left(U_{g}\right)
\end{gathered}
$$




$$
Q\left(n_{\mathrm{eff}}\right)=\frac{U_{g}}{S} Y_{p+1}\left(U_{g} / S\right) J_{p}(U)-U J_{p+1}(U) Y_{p}\left(U_{g} / S\right),
$$

then the solutions $n_{\mathrm{eff}}$ are determined by the dispersion equation

$$
M N-P Q=0 .
$$

Solutions of Equation (10.9) may or may not exist, depending on the parameters. Their (finite) number depends also on these parameters. Standard numerical procedures allow one to extract the number of solutions and the effective indices corresponding to each of the modes. For a given mode, $n_{\text {eff }}$ being known, the quantities $U, U_{g}$, and $W$ are known, and the constants $B, C, D$ can be computed from $A$, which can be used for normalization. Specifically, we have

$$
\begin{gathered}
B=\frac{\pi}{2}\left[U J_{p+1}(U) Y_{p}\left(U_{g} / S\right)-\frac{U_{g}}{S} J_{p}(U) Y_{p+1}\left(U_{g} / S\right)\right] \\
C=\frac{\pi}{2}\left[\frac{U_{g}}{S} J_{p+1}\left(U_{g} / S\right) J_{p}(U)-U J_{p+1}(U) J_{p}\left(U_{g} / S\right)\right] \\
D=\frac{B J_{p}\left(U_{g}\right)+C Y_{p}\left(U_{g}\right)}{K_{p}(W)} .
\end{gathered}
$$

It is possible to find parameters such that an LP mode has a structure comparable to an LG mode. We show a specific (but somewhat arbitrary) example in Figure 67, for which the Hermitian scalar product of the fiber $\left(\mathrm{LP}_{5,5}\right)$ mode with an $\mathrm{LG}$ mode is about $75 \%$ in power. The parameters of the LP mode are

$$
\begin{aligned}
& r_{C}=4 \mu \mathrm{m}, r_{G}=58.99 \mu \mathrm{m}, n_{C}=1.452126, \\
& n_{G}=1.446846, n_{\mathrm{ext}}=1.4452, n_{\mathrm{eff}}=1.4452017
\end{aligned}
$$

The $\mathrm{LG}_{5,5}$ mode has $w=4.472 \mathrm{~cm}$. It is surely possible to have a better matching after an optimization study that we are planning. The last step is to couple a $\mathrm{TEM}_{0,0}$ laser beam with such a fiber and then the resulting fundamental LP mode to the $\mathrm{LP}_{5,5}$ via a Bragg coupler.

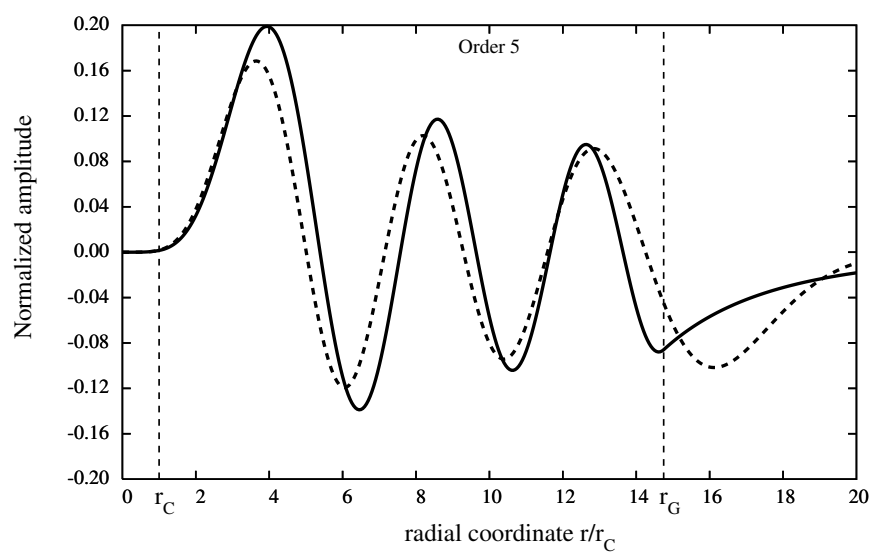

Figure 67: Mode $\mathrm{LP}_{5,5}$ (solid line), Mode $\mathrm{LG}_{5,5}$ (dashed line) 


\section{Conclusion}

We have discussed some of the main issues regarding mirrors to be used in a high-optical-power interferometer. These issues are thermal lensing, thermal aberration, thermal noise and thermoelastic noise. These spurious effects do not act at the same level. Thermal issues arise directly from the laser power and make necessary compensation systems more and more difficult as the power increases. Thermal noises (standard and thermoelastic) are not related to the laser power, but dominate the shot noise in the central spectral region, spoiling any gain of sensitivity expected from a higher laser power. An interesting approach to reduce these effects is to change the readout beam from the fundamental $\mathrm{TEM}_{0,0}$ currently used, to the more widely spread ("exotic") light power distributions, (either mesa, conical or high-order Laguerre-Gauss). We have given the formulas for estimating the gains with respect to the above cited issues for these different modes. Thus, we hope to contribute to the design of advanced instruments. It is already possible to point out that exotic beams provide a high gain (up to a factor of five) in thermal noise and thermoelastic noise and a huge gain in spurious thermal effects (up to two orders of magnitude).

\section{Acknowledgment}

I am indebted to Kip S. Thorne for his extensive review work, and for a huge number of suggestions among which I tried to address as much as I could. 


\section{References}

[1] Abramowitz, M., and Stegun, I.A., eds., Handbook of Mathematical Functions: with Formulas, Graphs, and Mathematical Tables, (Dover, New York, 1972). 3.1.1

[2] Agresti, J., Researches on Non-standard Optics for Advanced Gravitational Waves Interferometers, Ph.D. Thesis, (University of Pisa, Pisa, 2007). [arXiv:0806.3065v1]. 9.2.2

[3] Bondarescu, B., and Thorne, K.S., "New family of light beams and mirror shapes for future LIGO interferometers", Phys. Rev. D, 74, 082003, 1-6, (2006). [DOI], [gr-qc/0409083]. 2.2

[4] Bondarescu, M., Kogan, O., Chen, Y., Lundgreen, A., Bondarescu, R., and Tsang, D., "Beams of the Future", Joint LSC/VIRGO Meeting, Hanover, Germany, October 22-26, 2007, conference paper, (2007). Related online version (cited on 22 July 2008):

http://www.ligo.caltech.edu/docs/G/G070767-00/. 2.3.2, 8.3.2

[5] Bondu, F., Hello, P., and Vinet, J.-Y., "Thermal noise in mirrors of interferometric gravitational wave antennas", Phys. Lett. A, 246, 227-236, (1998). [DOI]. 8.2, 8.2, 8.4.2

[6] Born, M., and Wolf, E., Principles of Optics: Electromagnetic Theory of Propagation, Interference and Diffraction of Light, (Cambridge University Press, Cambridge; New York, 2002), 7th exp. edition. [Google Books]. 3.4

[7] Braginsky, V.B., Gorodetsky, M.L., and Vyatchanin, S.P., "Thermodynamical fluctuations and photo-thermal shot noise in gravitational wave antennae", Phys. Lett. A, 264, 1-10, (1999). [DOI], [cond-mat/9912139]. 9.2.1

[8] Braginsky, V.B., and Vyatchanin, S.P., "Thermodynamical fluctuations in optical mirror coatings", Phys. Lett. A, 312, 244-255, (2003). [DOI], [cond-mat/0302617]. 9.2.4

[9] Callen, H.B., and Welton, T.A., "Irreversibility and Generalized Noise", Phys. Rev., 83, 3440, (1951). [DOI]. 8.1

[10] Courty, J.-M., Heidmann, A., and Pinard, M., "Quantum Locking of Mirrors in Interferometers", Phys. Rev. Lett., 90, 083601, 1-4, (2003). [DOI], [gr-qc/0212081]. 1

[11] D'Ambrosio, E., "Nonspherical mirrors to reduce thermoelastic noise in advanced gravitational wave interferometers", Phys. Rev. D, 67, 102004, 1-16, (2003). [DOI]. 1, 2.2

[12] D'Ambrosio, E., O'Shaughnessy, R., Strigin, S., Thorne, K.S., and Vyatchanin, S.P., "Reducing Thermoelastic Noise in Gravitational-Wave Interferometers by Flattening the Light Beams", arXiv e-print, (2004). [gr-qc/0409075]. 2.2

[13] Degallaix, J., Zhao, C., Ju, L., and Blair, D.G., "Simulation of bulk-absorption thermal lensing in transmissive optics of gravitational waves detectors", Appl. Phys. B, 77, 409-414, (2003). [DOI]. 4

[14] Degallaix, J., Zhao, C., Ju, L., and Blair, D.G., "Thermal lensing compensation for AIGO high optical power test facility", Class. Quantum Grav., 21, S903-S908, (2004). [DOI]. 4

[15] Di Paulo Emilio, M., personal communication. 4.3

[16] Durnin, J., Miceli Jr, J.J., and Eberly, J.H., "Diffraction-Free Beams", Phys. Rev. Lett., 58, 1499-1501, (1987). [DOI]. 2.3.1 
[17] Fejer, M.M., Rowan, S., Cagnoli, G., Crooks, D.R.M., Gretarsson, A.M., Harry, G.M., Hough, J., Penn, S.D., Sneddon, P.H., and Vyatchanin, S.P., "Thermoelastic dissipation in inhomogeneous media: loss measurements and displacement noise in coated test masses for interferometric gravitational wave detectors", Phys. Rev. D, 70, 082003, (2004). [DOI], [gr-qc/0402034]. 9.2 .4

[18] Harry, G.M., Gretarsson, A.M., Saulson, P.R., Kittelberger, S.E., Penn, S.D., Startin, W.J., Rowan, S., Fejer, M.M., Crooks, D.R.M., Cagnoli, G., Hough, J., and Nakagawa, N., "Thermal noise in interferometric gravitational wave detectors due to dielectric optical coatings", Class. Quantum Grav., 19, 897-917, (2002). [DOI], [gr-qc/0109073]. 8.3.1

[19] Hello, P., Modele physique et simulation de l'antenne interferometrique gravitationnelle Virgo, Ph.D. Thesis, (Université Paris-Sud, Orsay, 1990). 5.1

[20] Hello, P., and Vinet, J.-Y., "Analytical models of thermal aberrations in massive mirrors heated by high power laser beams", J. Phys. France, 51, 1267-1282, (1990). [DOI]. 3

[21] Hello, P., and Vinet, J.-Y., "Analytical models of transient thermoelastic deformations of mirrors heated by high power cw laser beams", J. Phys. France, 51, 2243-2261, (1990). [DOI]. 3

[22] Ju, L., Aoun, M., Barriga, P., Blair, D.G., Brooks, A., Burman, R., Burston, R., Chin, X.T., Chin, E.J., Lee, C.Y., Coward, D., Cusack, B., de Vine, G., Degallaix, J., Dumas, J.C., Garoi, F., Gras, S., Gray, M., Hosken, D.J., Howell, E., Jacob, J.S., Kelly, T.L., Lee, B., Lee, K.T., Lun., T., McClelland, D.E., Mow-Lowry, C.M., Mudge, D., Munch, J., Schediwy, S., Scott, S., Searle, A., Sheard, B., Slagmolen, B.J.J., Veitch, P.J., Winterflood, J., Woolley, A., Yan, Z., and Zhao, C., "ACIGA's high optical power test facility", Class. Quantum Grav., 21, S887-S893, (2004). [DOI]. 4

[23] Katz, J.I., "Temperature Dependence of the Index of Refraction of Fused Silica. Answer to Question \#50", Am. J. Phys., 65, 942-943, (1997). [DOI], [cond-mat/9712327]. 3.2

[24] Landau, L.D., and Lifshitz, E.M., Theory of Elasticity, Course of Theoretical Physics, vol. 7, (Pergamon Press, Oxford; New York, 1986), 3rd edition. 9.1, 9.1

[25] Lawrence, R., Ottaway, D., Zucker, M., and Fritschel, P., "Active correction of thermal lensing through external radiative thermal actuation", Opt. Lett., 29, 2635-2637, (2004). [DOI]. 4

[26] Levin, Y., "Internal thermal noise in the LIGO test masses: A direct approach", Phys. Rev. $D, \mathbf{5 7}, 659-663,(1998)$. [DOI], [gr-qc/9707013]. 8.1

[27] "LIGO Laboratory Home Page", project homepage, California Institute of Technology. URL (cited on 10 July 2008):

http://www.ligo.caltech.edu/. 1

[28] Liu, Y.T., and Thorne, K.S., "Thermoelastic noise and homogeneous thermal noise in finite sized gravitational-wave test masses", Phys. Rev. D, 62, 122002, 1-10, (2000). [DOI], [grqc/0002055]. 8.4, 8.4.2, 8.4.2, 9.1, 9.1, 9.2.1

[29] Lovelace, G., "The dependence of test-mass thermal noises on beam shape in gravitationalwave interferometers", Class. Quantum Grav., 24, 4491-4512, (2007). [DOI], [gr-qc/0610041]. 9.2.4, 9.2.4

[30] Lück, H., Freise, A., Goßler, S., Hild, S., Kawabe, K., and Danzmann, K., "Thermal correction of the radii of curvature of mirrors for GEO 600", Class. Quantum Grav., 21, S985-S989, (2004). [DOI]. 4 
[31] Mours, B., Tournefier, E., and Vinet, J.-Y., "Thermal noise reduction in interferometric gravitational wave antennas: using high order TEM modes", Class. Quantum Grav., 23, 5777-5784, (2006). [DOI]. 1

[32] Nakagawa, N., "Thermal noise in half-infinite mirrors with nonuniform loss: A slab of excess loss in a half-infinite mirror", Phys. Rev. D, 65, 102001, (2002). [DOI], [gr-qc/0105046]. 8.3.1

[33] O'Shaughnessy, R., Strigin, S., and Vyatchanin, S.P., "The implications of Mexican-hat mirrors: calculations of thermoelastic noise and interferometer sensitivity to perturbation for the Mexican-hat-mirror proposal for advanced LIGO", arXiv e-print, (2004). [gr-qc/0409050]. 2.2

[34] Rowan, S., Cagnoli, G., Sneddon, P.H., Hough, J., Route, R., Gustafson, E.K., Fejer, M.M., and Mitrofanov, V., "Investigation of mechanical loss factors of some candidate materials for the test masses of gravitational wave detectors", Phys. Lett. A, 265, 5-11, (2000). [DOI]. 1

[35] Savov, P., and Vyatchanin, S., "Estimate of tilt instability of mesa-beam and Gaussian-beam modes for advanced LIGO", Phys. Rev. D, 74, 082002, 1-10, (2006). [DOI], [gr-qc/0409084]. 2.2

[36] Somiya, K., and Yamamoto, K., "Coating thermal noise of a finite-size cylindrical mirror", Phys. Rev. D, 79, 102004, (2009). [DOI], [arXiv:0903.2902v1]. 9.2.5

[37] Thorne, K.S., "Gravitational radiation", in Hawking, S.W., and Israel, W., eds., Three Hundred Years of Gravitation, pp. 330-458, (Cambridge University Press, Cambridge; New York, 1987). [Google Books]. 1

[38] Timoshenko, S., and Goodier, J.N., Theory of Elasticity, (McGraw-Hill, New York, 1951), 2nd edition. 3.3.1

[39] Uchiyama, T., Tatsumi, D., Tomaru, T., Tobar, M.E., Kuroda, K., Suzuki, T., Sato, N., Yamamoto, A., Haruyama, T., and Shintomi, T., "Cryogenic cooling of a sapphire mirrorsuspension for interferometric gravitational wave detectors", Phys. Lett. A, 242, 211-214, (1998). [DOI]. 1

[40] Vinet, J.-Y., "Mirror thermal noise in flat-beam cavities for advanced gravitational wave interferometers", Class. Quantum Grav., 22, 1395-1404, (2005). [DOI]. 1

[41] "Virgo", project homepage, INFN. URL (cited on 10 July 2008):

http://www.virgo.infn.it/. 1

[42] Willems, P., "Thermal Compensation in LIGO", Joint LSC/VIRGO Meeting, Baton Rouge, LA, March 19-22, 2007, conference paper, (2007). Related online version (cited on 22 July 2008):

http://www.ligo.caltech.edu/docs/G/G070146-00/. 4

[43] Zhao, C., Degallaix, J., Ju, L., Fan, Y., Blair, D.G., Slagmolen, B.J.J., Gray, M.B., Mow Lowry, C.M., McClelland, D.E., Hosken, D.J., Mudge, D., Brooks, A., Munch, J., Veitch, P.J., Barton, M.A., and Billingsley, G., "Compensation of Strong Thermal Lensing in HighOptical-Power Cavities", Phys. Rev. Lett., 96, 231101, (2006). [DOI], [gr-qc/0602096v2]. 4 